Dr. 2234

\title{
LA-8592-PR
}

Progress Report

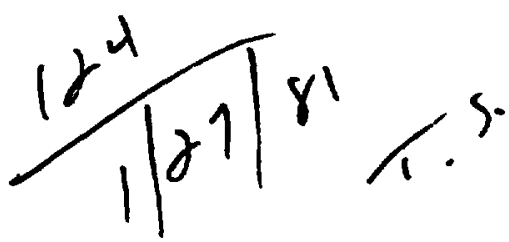
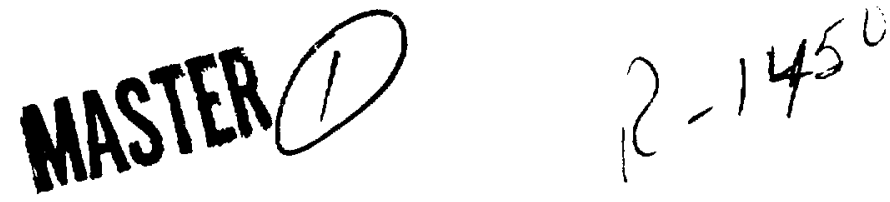

Accelerator Technology Program January-December 1979

$\frac{\pi}{5}$
$\frac{0}{0}$
$\frac{0}{0}$
$\frac{\pi}{0}$
$\frac{2}{5}$
$\frac{2}{5}$ 
LA-8592-PR

Progress Report

UC-28

Issued: November 1980

\title{
Accelerator Technology Program
}

\section{January-December 1979}

Compiled by

\author{
E. A. Knapp
}

R. A. Jameson

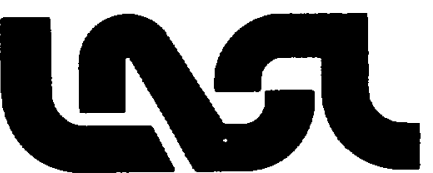




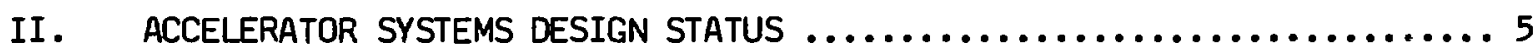

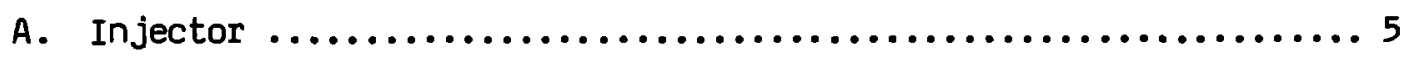

B. Radio-Frequency Quadrupole (RFQ) Accelerator ................6 6

1. Evolution of Theories and Analytical Software $\ldots \ldots \ldots \ldots \ldots . .7$

2. RFQ Proof-of-Principle (POP) Beam-Dynamics Design ..........10

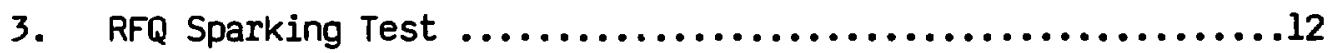

4. Preliminary FMIT RFQ Beam-Dynamics Designs $\ldots \ldots \ldots \ldots \ldots \ldots 12$

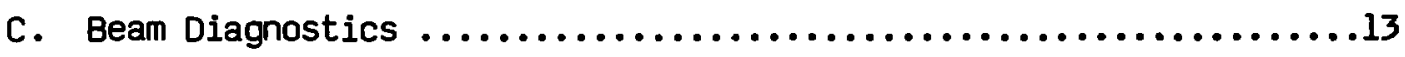

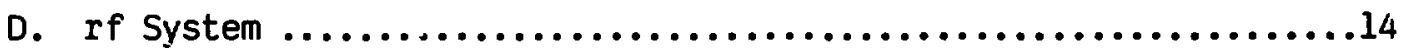

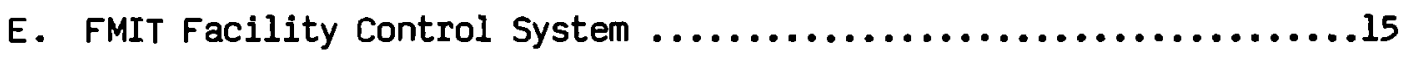

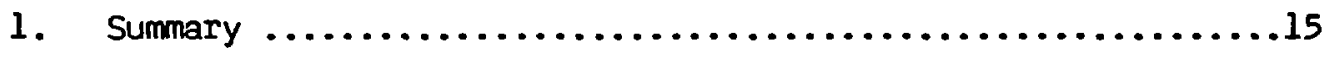

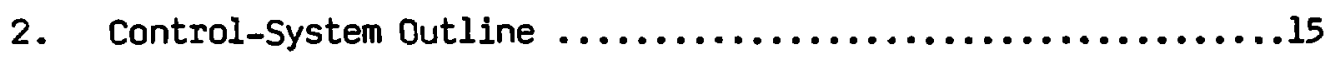

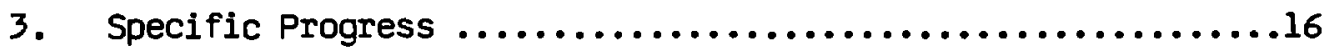

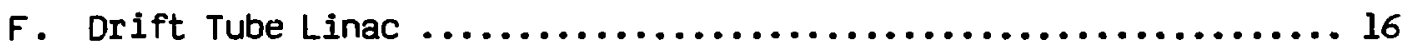

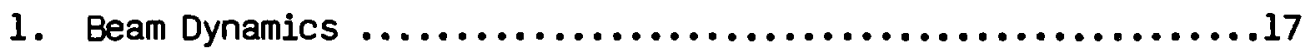

2. Linac Tank ..................................17

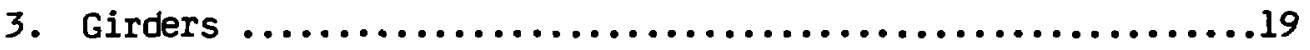

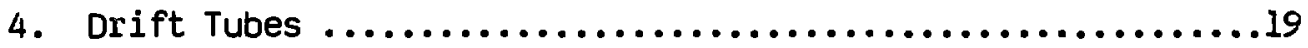

5. Drift Tube Quadrupole Magnets .................... 19

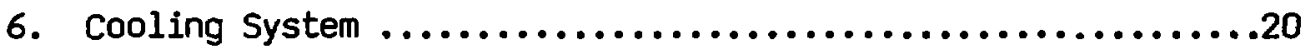

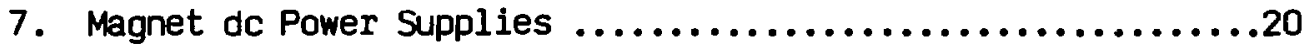

8. Vacuum System $\ldots \ldots \ldots \ldots \ldots \ldots \ldots \ldots \ldots \ldots \ldots \ldots \ldots \ldots \ldots \ldots$.

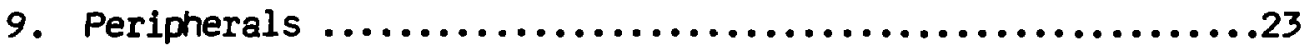

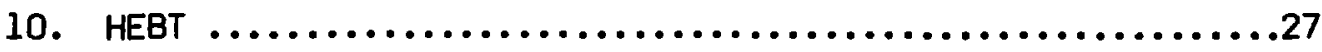

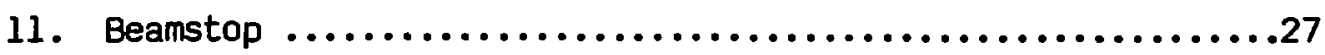




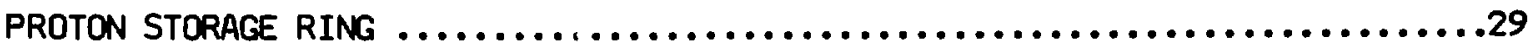

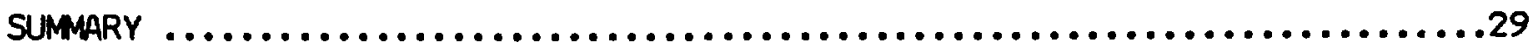

I. ACTIVE COMPONENT DEVELOPMENT $\ldots \ldots \ldots \ldots \ldots \ldots \ldots \ldots \ldots \ldots \ldots \ldots \ldots \ldots \ldots \ldots \ldots$

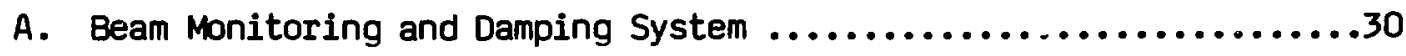

B. Multipactoring Driver..................................

C. The 2.8-MHz Buncher System .............................

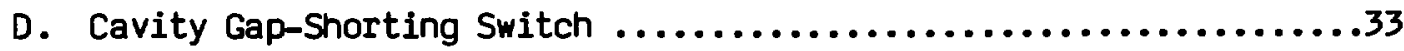

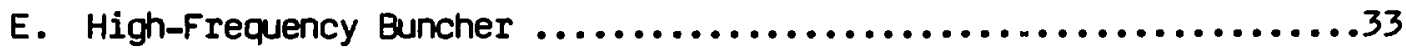

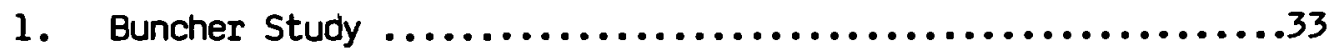

2. Disk-and-Washer (DAW) Structure for High-Frequency Buncher ..37

F. Pulsed Power . .........................................38

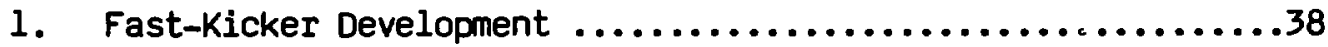

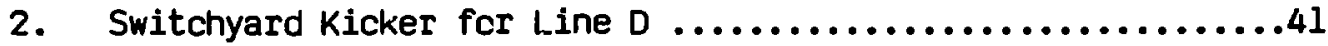

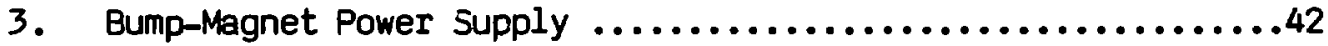

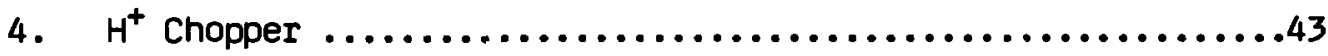

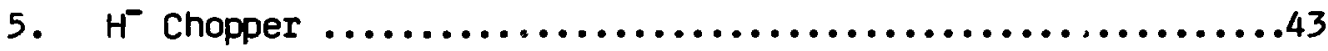

II. PASSIVE COMPONENTS AND ALIGNMENT $\ldots \ldots \ldots \ldots \ldots \ldots \ldots \ldots \ldots \ldots \ldots \ldots \ldots \ldots$

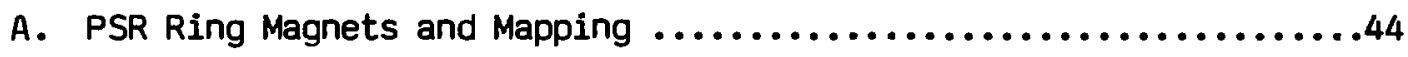

B. Stripper Magnet .....................................44

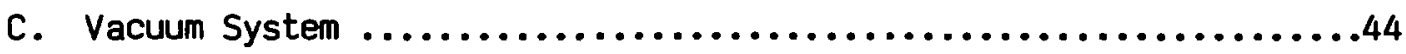

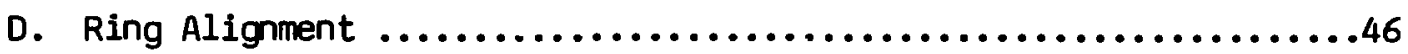

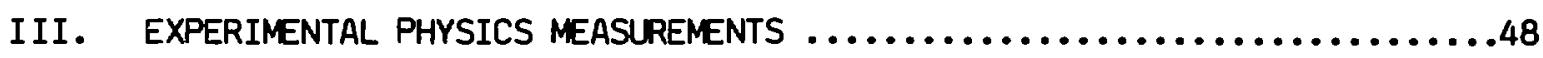

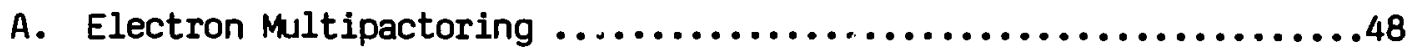

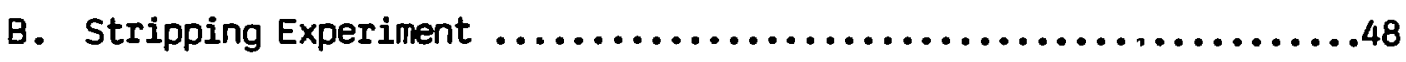

C. Time-of-Flight Energy Measurements $\ldots \ldots \ldots \ldots \ldots \ldots \ldots \ldots \ldots . \ldots . \ldots \ldots$

IV. THEORETICAL AND PRACTICAL CALCULATIONS $\ldots \ldots \ldots \ldots \ldots \ldots \ldots \ldots \ldots \ldots$

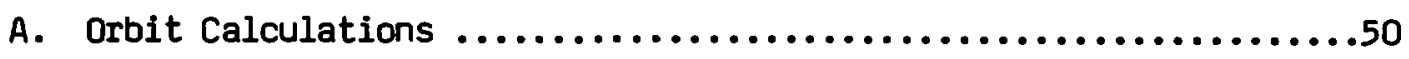

1. Orbits in the PSR with Ideal Magnets $\ldots \ldots \ldots \ldots \ldots \ldots \ldots \ldots . .50$

2. Transfer Map for Median-Plane Motion in Bending Magnets Having Either Parallel faces or Normal Entry and Exit .......51 
3. Orbits in the Imperfect PSR ........................

4. Beam-Resonance Effects .................................

B. PSF Beam-Induced Desorption . ............................ . . . . . . .

1. Beam-Induced Desorption Yields ...................... . . . 54

2. Cleaning by Beam Desorption ....................... 54

3. Methods of Increasing Critical Current ................55

4. Conclusions Affecting Vacuum-System Design .............56

C. Stripper-Foil Injection Dynamics .......................57

D. Beam Transport ...................................66

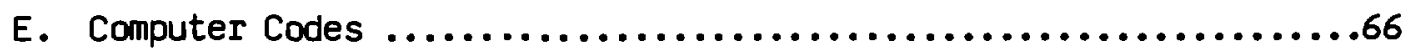

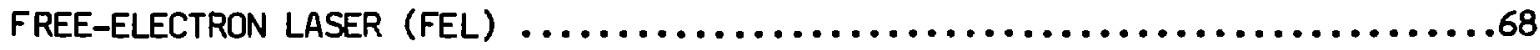

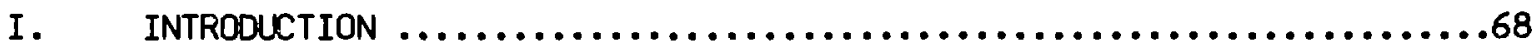

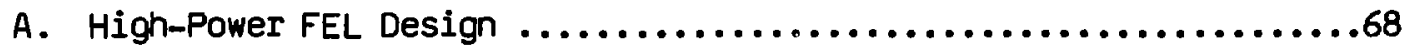

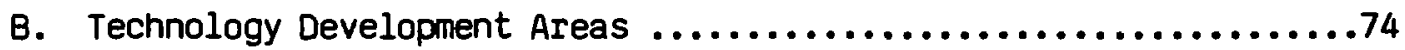

C. Objectives of the Present Program.........................77

II $\quad$ THEORY $\ldots \ldots \ldots \ldots \ldots \ldots \ldots \ldots \ldots \ldots \ldots \ldots \ldots \ldots \ldots \ldots \ldots \ldots \ldots \ldots \ldots \ldots \ldots \ldots \ldots \ldots$

A. Single-Particle Theory $\ldots \ldots \ldots \ldots \ldots \ldots \ldots \ldots \ldots \ldots \ldots \ldots \ldots \ldots \ldots$

B. Multiparticle Theory ....................................79

C. Optimization of Laser Performance ...................... 81

D. Effects of Transverse Electron Momentum ..................... 84

III. EXPERIMENTAL DESIGN $\ldots \ldots \ldots \ldots \ldots \ldots \ldots \ldots \ldots \ldots \ldots \ldots \ldots \ldots \ldots \ldots \ldots$

A. Accelerator Modifications ...............................89

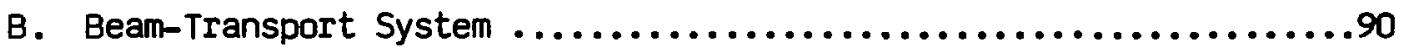

C. Wiggler Design .....................................92

D. Laser Oscillator and Amplifier .........................93

E. Laser Diagnostics ...................................94

F. Impact of Optical Damage on the Design ...................97

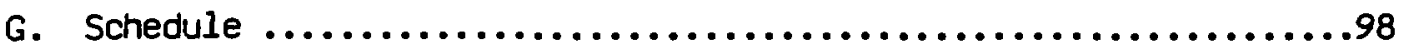

IV. CONCLUSIONS AND RECOMMENDATIONS $\ldots \ldots \ldots \ldots \ldots \ldots \ldots \ldots \ldots \ldots \ldots$ 


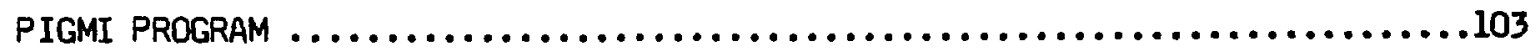

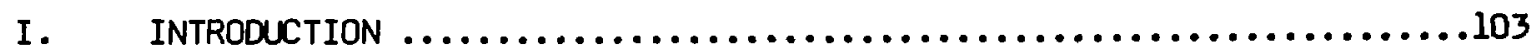

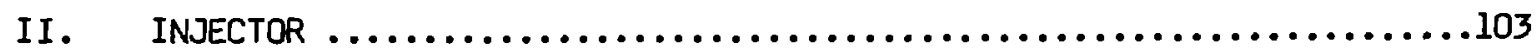

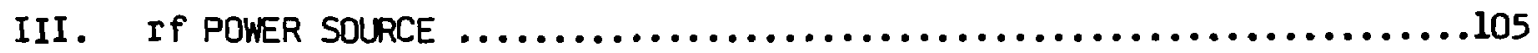

IV. INSTRUMENTATION AND CONTROL SYSTEM $\ldots \ldots \ldots \ldots \ldots \ldots \ldots \ldots \ldots \ldots \ldots \ldots$

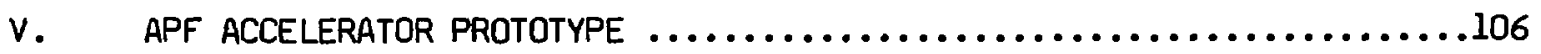

vI. DTL SECTION $\ldots \ldots \ldots \ldots \ldots \ldots \ldots \ldots \ldots \ldots \ldots \ldots \ldots \ldots \ldots \ldots \ldots \ldots \ldots \ldots \ldots \ldots$

VII. PERMANENT-MAGNET QUACRUPOLES $\ldots \ldots \ldots \ldots \ldots \ldots \ldots \ldots \ldots \ldots \ldots \ldots \ldots \ldots \ldots \ldots \ldots$

VIII. DEVELOPMENT OF THE RADIO-FREQUENCY QUADRLPOLE STRUCTURE $\ldots \ldots \ldots \ldots \ldots 113$

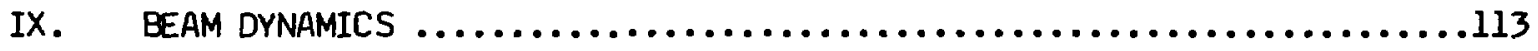

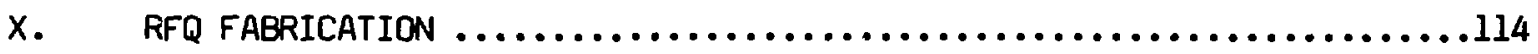

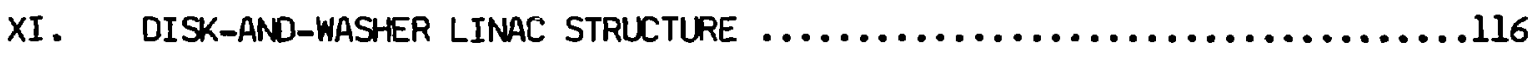

XII. BRIDGE COUPLERS FOR THE DAW LINAC STRUCTURE $\ldots \ldots \ldots \ldots \ldots \ldots \ldots \ldots 118$

XIII. FIELD GRADIENT AND AVERAGE POWER ACCELERATOR STRUCTURE TEST $\ldots \ldots \ldots \ldots 123$

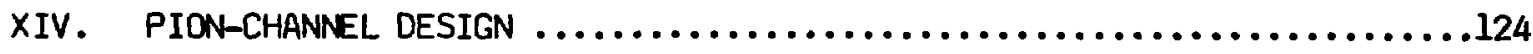

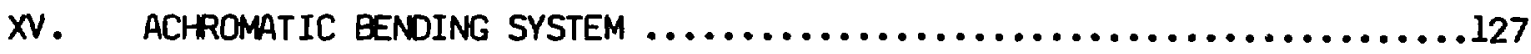




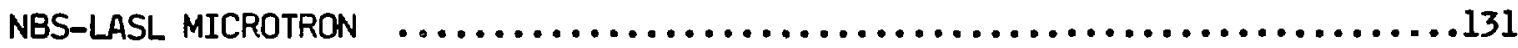

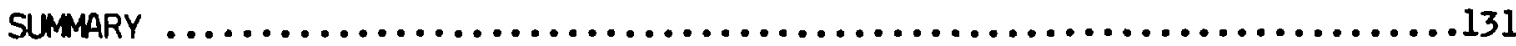

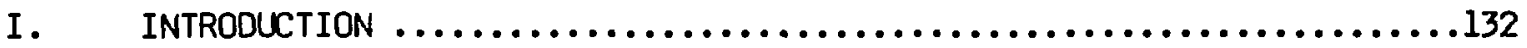

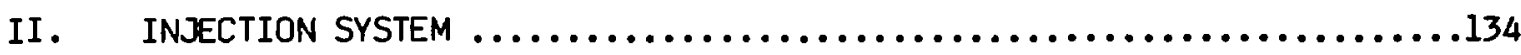

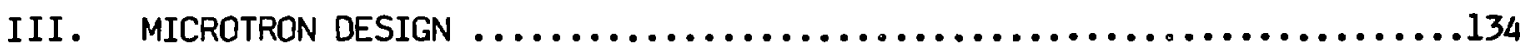

IV. DISK-AND-WASHER ACCELERATING STRUCTURE $\ldots \ldots \ldots \ldots \ldots \ldots \ldots \ldots \ldots \ldots \ldots$

HEAVY ION FUSION ACCELERATOR DEVELOPMENT $\ldots \ldots \ldots \ldots \ldots \ldots \ldots \ldots \ldots \ldots \ldots \ldots \ldots$

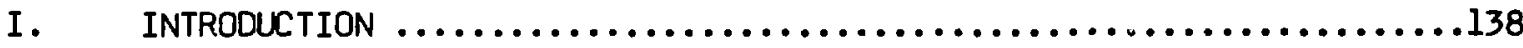

I I. LINEAR ACCELERATOR CURRENT LIMITS $\ldots \ldots \ldots \ldots \ldots \ldots \ldots \ldots \ldots \ldots \ldots \ldots \ldots \ldots \ldots \ldots \ldots \ldots$

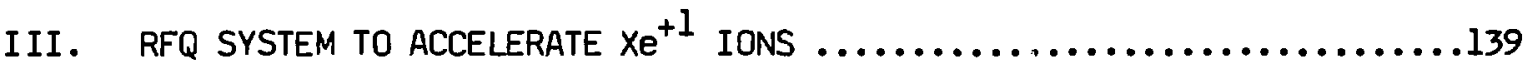

IV. MLTI-CHANNEL HEAVY-ION ACCELERATORS AND FUNNELING TECHNIQUES $\ldots \ldots \ldots 140$

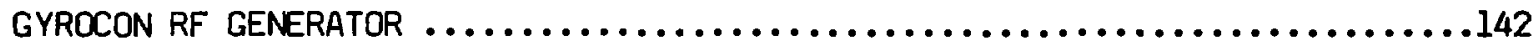

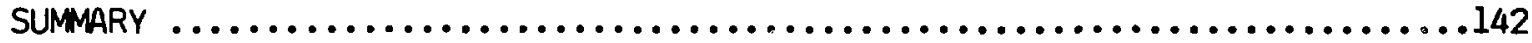

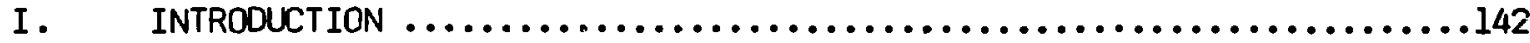

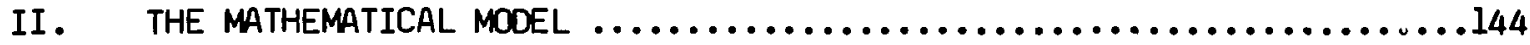

III. CALCULATION OF RADIAL-STYLE GYROCON PERFORMANCE $\ldots \ldots \ldots \ldots \ldots \ldots \ldots 146$

A. The Calculation Method ...................................

B. Electron Dynamics ....................................147

C. Limits to Radial Gyrocon Performance ......................150 
IV. THE LASL GYROCON DEMONSTRATION TUBE .154

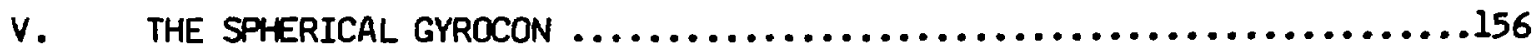

VI. PROBLEMS REMAINING AND SUGGESTIONS FOR FUTURE WORK $\ldots \ldots \ldots \ldots \ldots \ldots . . \ldots 3$

$\mathrm{H}^{-}$ION SOURCE, INJECTOR, AND ACCELERATOR TEST STAND STUDIES $\ldots \ldots \ldots \ldots \ldots \ldots 167$

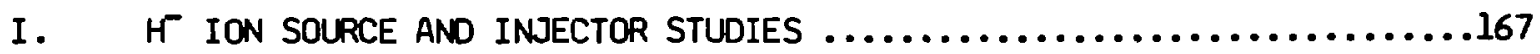

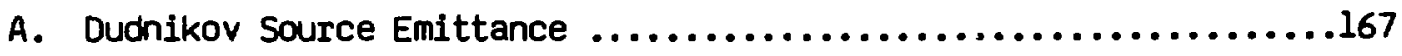

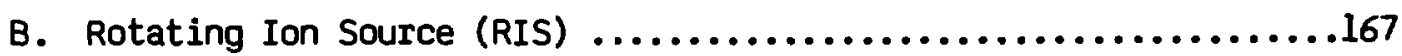

1. RIS-l Results and Critical Questions .................167

2. RIS Design and Improvements ......................168

3 RIS-1 Parametric Studies ...........................169

c. Cesium Flow Studies ................................172

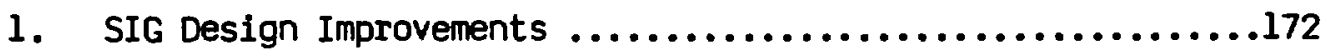

2. Cesium Flow Measurements for the Dudnikov and RIS Ion Sources ...............................173

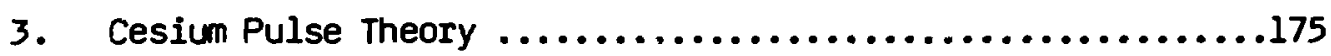

4. Evolution of Cesium from the $2 \mathrm{Ti}+\mathrm{Cs}_{2} \mathrm{Cr}_{2} \mathrm{O}_{7}$ Mixture $\ldots \ldots \ldots . .177$

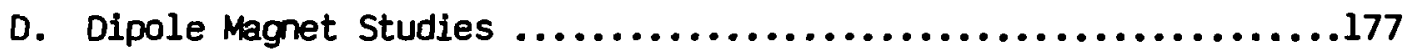

E. The $20-\mathrm{keV} \mathrm{H}$ Beam Transport $\ldots \ldots \ldots \ldots \ldots \ldots \ldots \ldots \ldots \ldots \ldots \ldots \ldots \ldots \ldots$

F. Accelerating Column Optics ............................179

1. First-Order Calculations ..........................179

2. Higher Order Calculations $\ldots \ldots \ldots \ldots \ldots \ldots \ldots \ldots \ldots \ldots \ldots$

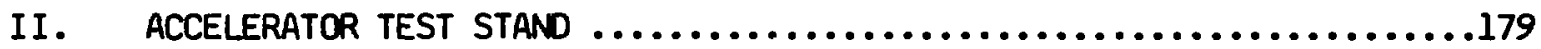

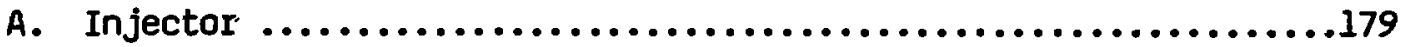

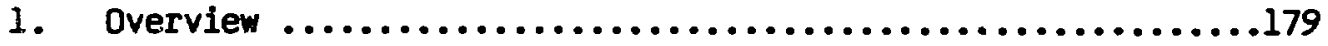

2. Source Design ..................................181

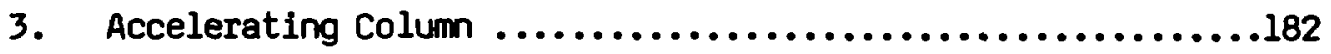

B. Beam Transport Between the Buncher and Accelerator $\ldots \ldots \ldots \ldots \ldots . .182$

C. Laser Diagnostic Development .........................183

D. Three-Cell Test Stand Linac Cavity ........................187 


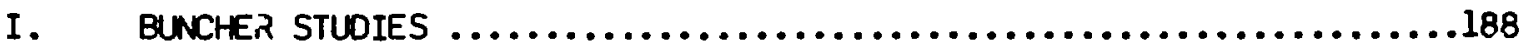

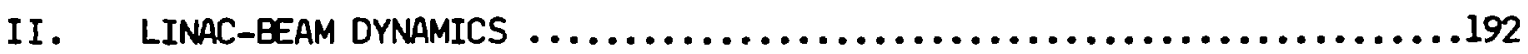

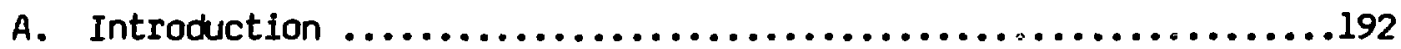

1. Accelerator Acceptance .............................193

2. Scaling Laws ..................................193

3. Matched Beam Parameters ............................193

4. Check on Particle Tracing Codes .......................193

B. Linear Smooth-Focusing and Discrete-Accelerating Gaps ...........194

C. Different $x$ and $y$ Emittances .............................194

D. Phase-Space Distributions Matched to External Nonlinearities ....195

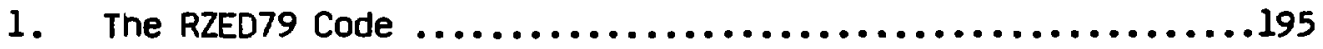

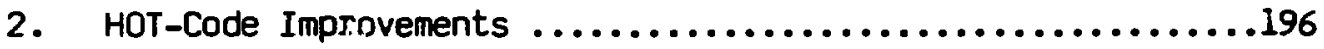

3. Distribution Properties with $z^{3}$ Terms $\ldots \ldots \ldots \ldots \ldots \ldots \ldots . \ldots 197$

4. ATS Distributions ..................................

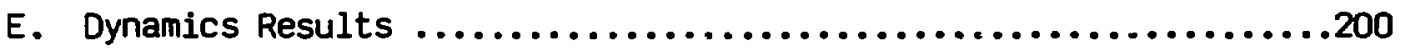

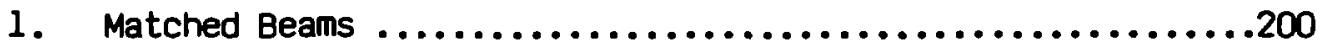

2. If Coupling and Emittance Growth ..................202

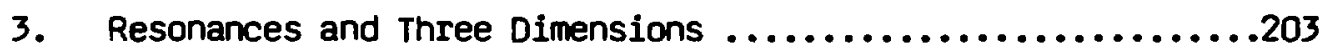

F. Discussion ....................................206

III. THE STARK EFFECT AS A TOOL FOR MEASURING If ELECTRIC FIELDS $\ldots \ldots \ldots . .206$ 


\title{
ACCELERATOR TECHNOLOGY PROGRAM
}

\author{
Compiled by \\ E. A. Knapp and R. A Jameson
}

\begin{abstract}
The activities of Los Alamos Scientific Laboratory's (LASL) Accelerator Technology (AT) Division during the calendar year 1979 are highlighted, with references to more detailed reports. This report is organized around the major projects of the Division, reflecting a wide variety of applications and sponsors.

The first section covers the Fusion Materials Irradiation Test program, a collaborative effort with the Hanford Engineering Development Laboratory; the second section summarizes progress on the Proton Storage Ring to be built between LAMPF and the LASL Pulsed Neutron Research facility. A new project that achieved considerable momentum during the year is described next--the free-electron laser studies; the following section discusses the status of the Pion Generator for Medical Irradiation program. Next, two more new programs, the racetrack microtron being developed jointly by AT-Division and the National Bureau of Standards and the radiofrequency ( $r f$ ) accelerator development for heavy ion fusion, are outlined. Development activities on a new type of high-power, high-efficiency $r$ amplifier called the gyrocon are then reported, and the final sections cover development of $\mathrm{H}^{-}$ion sources and injectors, and linear accelerator instrumentation and beam dynamics.
\end{abstract}

\section{SUMMARY}

LoS Alamos Scientific Laboratory's (LASL) Accelerator Technology (AT) Division is involved in innovation and improvement of linear and circular accelerator concepts and technology, and the application of this technology to a wide variety of needs. During this period, our activities ranged over fusion materials testing, a storage $r i n g$ for nuclear physics and materials research, free-electron laser systems that (when successfully demonstrated) will find 
very wide application, accelerators for cancer therapy, electron linac and microtron development, high-performance linear accelerator systems, advanced ion source and radio-frequency ( $r f$ ) power amplifier research, and accelerators for heavy-ion fusion. The programs are often highly symbiotic; advances in one area are often immediately applicable in others.

The preceding reports in this series cover Jan-Mar 1978 (LA-8218-PR) and Apr-Dec 1978 (LA-8350-PR).

Strong progress was made during this period on the Fusion Materials Irradiation Test (FMIT) program. The joint Hanford Engineering Development (HEDL)/LASL accelerator design team at LASL pushed the complete design well into the preliminary design stage. Office and laboratory space became available, and installation of utilities and some prototype equipment began. The injector test stand was used extensively during the latter part of the year. A great deal of emphasis was placed on the development of the new radiofrequency quadrupole (RFQ) structure, with participation on various aspects from throughout the Division and the Laboratory. The truly revolutionary potential of the concepts embodied in this device for accelerating low-velocity particles in a controllable and efficient manner has become very clear, and the first proof-of-principle tests with actual beam are eagerly awaited. The other engineering challenges of the FMIT accelerator have been attacked with equal vigor; considerable innovation has been required to provide rf power, drift-tube and beam-line structures, diagnostic and control systems suitable for the high-intensity, continuous operation and high availability needs of this facility.

The system requirements for the Proton Storage Ring (PSR) continued to evolve, with the present specifications set to provide $100_{-\mu \mathrm{A}}$ average current. The injection system matured into a double charge exchange using magnetic stripping from $\mathrm{H}^{-}$to $\mathrm{H}^{0}$, followed by foil stripping from $\mathrm{H}^{0}$ to $\mathrm{H}^{+}$, aided by actual stripping cross-section measurements performed at LAMPF. The fast beam monitoring, damping, and kicking systems received concentrated attention. A new type of vacuum flange seal was invented and developed. The report also presents detailed discussions of beam-induced desorption and stripper-foil design as applied to the PSR.

The new free-electron laser program completed a full conceptual design program to demonstrate first single-pass gain and then oscillation. The rationale for our approach, based on the advantages to system efficiency of 
nonuniform wigglers and energy recovery, is outlined, and the feasibility of high-power devices is shown. The objectives of the present program are then detailed, covering the development of theoretical and simulation tools as well as the experiment design and the proposed test program.

Wick on the Pion Generator for Medical Irradiation (PIGMI) program emphasized prototype tests with bean of the alternating phase-focused structure, and the development of the radio-frequency quadrupole and disk-and-washer accelerator structures.

A new program was initiated in collaboration with the National Bureau of Standards, Washington, DC, to determine the feasibility of a room-temperature racetrack microtron for high-energy, high-current, continuous beam electron acceleration. A prototype module of the ultimate requirement will be built at NBS to develop the required technology. This report details the conceptual design.

LASL became actively involved in the Heavy Ion Fusion program during this year, and participated extensively in workshops and meetings on this subject. AT-Division's interests centered on the application of the new RFQ structure for this progran; designs and techniques are outlined that offer remarkable advantages for accelerating low-velocity ions in single channels or in channel arrays.

Work continued on the gyrocon rf amplifier, a high-efficiericy, high-unitpower device. Considerable progress was made on the theoretical description and computer simulation of the beam dynamics in the tube; these results and their application to various parametric studies are given in this report. The experimental program to build an operating gyrocon is in the procurement and fabrication stage.

$\mathrm{H}^{-}$ion source injector development continued on fixed-electrode and rotating Dudnikov-type sources. Several parametric studies were completed, and the characteristics of cesium flow were investigated extensively.

A variety of analytical and simulation studies aimed at preserving very high beam quality during the accelerator process are described in the final sections of this report. Techniques for configuring multiple-harmonic buncher cavities to achieve desired longitudinal particle distributions are discussed. Last, but of fundamental importance, progress in understanding the basic causes of emittance growth observed in linacs is presented, in the framework of systematic studies of individual and combined effects. 


\section{FUSION MATERIALS IRRADIATION TEST PROJECT}

\section{INTRODUCTION}

In early 1978, LASL's AT-Division began a collaborative project with the Hanford Engineering Development Laboratory (HEDL) to build the Fusion Materials Irradiation Test (FMiT) facility at HEDL. LASL is designing a 35-MeV, 100-mA continuous duty factor deuteron accelerator for the facility. The deuteron beam will bombaro a flowing, molten-lithium target, which will emit an intense neutron beam. Samples of material for fusion-reactor components will be placed in this beam to study their properties under intense radiation. A team of LASL and HEDL personnel has been assembled at LASL to design the accelerator. A prototype accelerator, to 5-MeV energy, will be built at LASL.

Facilities for the FMIT project became available in 1979, with the irstallation of six transportables and the completion of the FMIT prototype building. These buildings provided an additional $7200 \mathrm{sq}$. $\mathrm{ft}$. of office space, $5000 \mathrm{sq}$. ft. of laboratory space, and $10000 \mathrm{sq} . \mathrm{ft}$. of experimental and equiprent area. This enabled the design team to be consolidated in one Tech area. Included in the 15 000-sq.-ft. prototype building are utility trenches, partitions, fire protection system, and all building utilities.

The power distribution system design was completed during 1979. The system includes a new substation, transformer, switchgear, and the building's interior power distribution. A total of 7.5 MVA will be provided, with 2.5 MVA at $440 \mathrm{~V}$ and 5 MVA at $13.8 \mathrm{kVA}$; the system can be upgraded to $12 \mathrm{MVA}$. Construction will begin in early 1980.

The cooling system's initial design has been completed, including a cooling tower and independent circuits for the heat exchangers, pumps and control valve. Construction will start in mid 1980.

Initial plans have been approved for a new $\$ 1.5 \mathrm{M}$ office building that will provide approximately $12000 \mathrm{sq}$. $\mathrm{ft}$. of building space. Design criteria were approved and the architect-engineer is to start design in early 1980, with construction to start in late 1980.

Several activities that wo would have done inside the prototype building had construction been corip lete were performed elsewhere. These projects included extensive tests on the injector preprototype test stand, low-power rf development work, linac-tank peripherals tests, and considerable experimental 
work on the RFQ. The occupancy of the prototype building now offers an opportunity to merge all these activities. On December 1 , some experimental activities began moving into the building.

Because of some delays in the fabrication of the beam-support stand for the low-energy beam transport (LEBT), the injector preprototype test stand was moved into the building. As soon as major components become available, the injector will be the first activity on the beam line.

Only very limited ac power is available now, although electricians have begun work on the interim phase-A power to permit injector operation for the next six months.

\section{I1. ACCELERATOR SYSTEMS DESIGN STATUS}

A. Injector

The injector group worked in parallel during the past year on R\&D, using the injector test stand, and on design of the prototype and FMIT accelerator components. ${ }^{1}$ Substantial progress resulted for the principal systems; that is, ion source, 100-kV extractor, high vacuum, species analysis, emittance measurement, ion-source microprocessor control, and high voltage.

An Osher reflex arc and three-cusp magnetic-field ion sources were evaluated. The Osher and MK II Cusp sources (cusp planes normal to the extraction axis) produce the required plasma density of $100 \mathrm{~mA} / \mathrm{cm}^{2}$. The Osher source has an instability of undetermined origin, and development has been stopped on it. The cusp source has a simple cubical construction and is very easy to operate. The cusp-source gas efficiency is $\sim 40 \%$, the $\mathrm{H}^{+}$ fraction is $46-56 \%$, and the stability is excellent. An oxide-coated nickelgauze cathode wrapped in cylindrical fashion about a 0.04-in. tantalum wire was developed and lasts for several tens of hours in dc operation of the cusp source.

The 100-kV extractor now is capable of 200-mA operation. An electron trap, consisting of an electrode at -1 to $-2 \mathrm{kV}$ placed between the ground electrode of the extractor and a ground electrode following the trap, not only prevented backstreaming of electrons but alsn gave nearly complete space-charge neutralization of the beam a very short distance downstream of the trap. The following additional modifications nearly eliminated sparkdowns of the extractor system: the pumping of the extractor gap was improved by an order of 
magnitude, the use of both baffles and magnets suppressed the electrons backstreaming through the pump-out holes, and the use of mild steel parts improved the extractor's magnetic shielding from the analyzing magnet stray fields.

A Freon-cooled baffle, followed by an oil diffusion pump using Santovac 5 pump fluid and a blower-forepump package, gave the clean high-speed pumping required.

The method of exciting the $90^{\circ}$ analyzing magnet was changed to an arrangement that minimizes ti.e stray field. The edge angles were increased, to change the focusing and to give an approximately circular spot at the magnet focus.

The beam emittance was measured, using two techniques: the first method used the divergence of the unanalyzed beam to give an upper bound of $0.05 \pi \mathrm{cm} \cdot \mathrm{mr}$ ad for the normalized emittance; the second method used a doublescanning device downstream of the analyzing magnet, and gave an upper limit of $0.1 \pi \mathrm{cm} \cdot \mathrm{mrad}$.

A Motorola 6800 microprocessor-based remote-control system has been in operation for the last quarter of the year. The display and microprocessorcontrol functions are provided by a display terminal; the ion-source control is from an associated panel. A fiber-optic light link communicates across the $100-k V$ interface.

Damage to the ion-source power supplies during high-voltage sparkdown of the extractor was eliminated by installing filters in the power 1 ines between the isolation transformer and the ion-source equipment dome.

B. Radio-Frequency Quadrupole Accelerator (RFQ)

The first linear accelerator structure following the dc irijector is the RFQ whose function is to accept the $0.10 \mathrm{MeV}$ unbunched deuteron beam from the injector, then bunch and accelerate it to an energy of $2.0 \mathrm{MeV}$. This energy is suitable for injection into the drift-tube linear accelerator (1inac) that accelerates the deuterons to their final energy. The RFQ is a new concept that has not heretofore been used outside the USSR. ${ }^{2,3}$ It promises to fulfill the above functions with little loss of beam intensity and with minimal beam-emittance increase. In addition to the description given here, certain aspects of RFQ bean dynamics are discussed in the section on heavy ion fusion. During the past year, a great deal of attention focused on the development program for the RFQ accelerator. Colleagues from many countries showed 
their interest, both at conferences and in their visits. The quantity and quality of effort invested in this program has resulted in clear progress on all fronts. Advances were in four development phases: (1) the evolution of theories and analytical software, (2) concepts and design, (3) completed hardware, and (4) experimental verification.

1. Evolution of Theories and Analytical Software. The RFQ generates time-varying electric quadrupole fields that provide radial-focusing forces to contain the ion motion. In contrast to the magnetic force that is proportional to ion velocity, the electric force is velocity independent and is particularly useful in focusing low-velocity ions. The RFQ electric quadrupole fields are generated by producing opposite polarity voltages on two pairs of conducting poles that surround the z-axis. The time-varying voltages are produced by $r f$ currents that flow in the transverse direction. Figure $i$ is a schematic drawing of the type of RFQ resonator that will be used in FMIT (the 4-vane resonator). If the pole tips do not vary in radius, the structure provides only radial focusing. If the pole tips are modulated in radius, as shown in Fig. 1 , they also produce longitudinal electric fields. These longitudinal fields are used to bunch and accelerate the ion beam. By appropriately varying the magnitude of the pole-tip modulation, the longitudinal fields cain he gradually increased from zero at the input of the RFQ to their final value near the RFQ output. In this way the ions are captured and bunched with high efficiency, and during the acceleration oniy a small percentage is lost by striking the RFQ poles.

The study of this new structure required the creation of tools for that

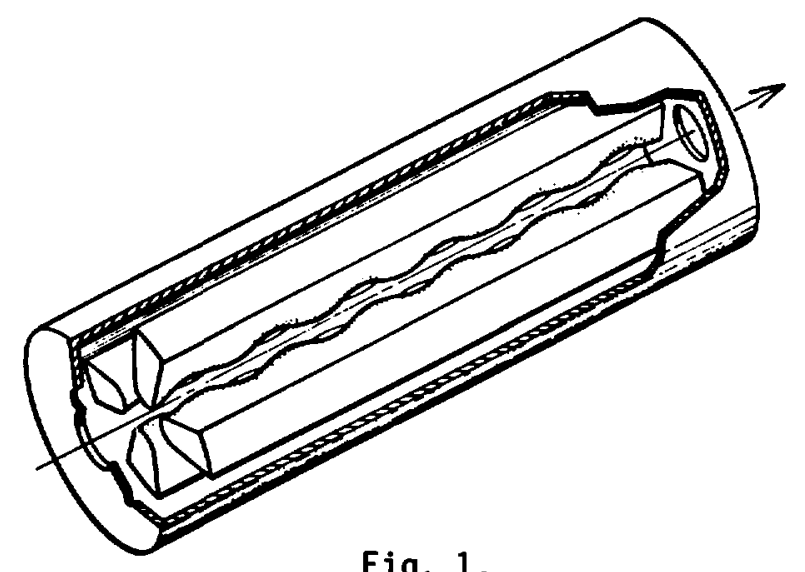

Fig. 1 . purpose. Early in the year, the only beam-dynamics tools available were the original Kapchinskii equations. By March, however, a version of PARMILA was modified to specifically model the RFQ. ${ }^{4,5}$

As a basis for this, we chose to use the lowest order potential function from which RFQ electric fields can be obtained in the quasiSchematic view of the 4-vane resonator. static approximation. This potential 
function is 30, obeys Laplace's equation, and contains only the lowest order Fourier component of the longitudinal-space dependence. The quasi-static approximation is well fulfilled because the RFQ aperture and the pole-tip radii are very small, compared to the $\lambda / 2$ conductor length in the transverse plane between two adjacent pole tips.

Two important and self-consistent quantities are calculated from the potential function: first, by performing the gradient operation, we obtain a complete 3D description of the electric fields; second, by setting the potential function equal to a constant, we obtain a 30 description of isopotential surfaces that are ther. the shapes of the conducting pole tips required to produce the electric fields. In practice, the poles are constructed to have the pole-tip radius exactly equal to that specified by the potential function. In the transverse plane, at each value of $z$, the pole tips are made to rave a constant radius of curvature specified by the potential function for the pole tip. This is a sufficiently good approximation to the exact hyperbola-like shape. The machining of the pole tips with a computer-controlled milling machine is discussed below.

The RFQ electric fields obtained from the potential function were used to construct transformations for the 60 phase space, consisting of the coordinates $x, x^{\prime}, y ; y^{\prime}, \phi$, and $W$. These transformations, as well as a spacecharge impulse, were incorporated into a beam-dynamics program called PARMTEQ. This program has been extensively used to analyze the performance of many RFQ accelerator designs. Usually the input ion beam is described as a random distribution of particles in the 40 phase-space $x, x^{\prime}, y$, and $y^{\prime}$. In addition, the input particles are usually assumed to be monoenergetic and also to be uniformly distributed in phase through $360^{\circ}$ to represent a dc beam. For a specified beam current the beam particles are transformed through the array of cells that represents the RFQ accelerator. Particles that strike the vanes or fall out of synchronism are eliminated and the radial and longitudinal phasespace distributions of the transmitted beam are generated at the output.

Different parameter surveys produced hundreds of RFQ designs. The variables included beam-channel length, capture efficiency, and field and phase slopes. By midyear, an essentially complete beam dynamics package for the RFQ was in hand. Two questions were brought to light by the analysis: could an RFQ 
be built in view of the very tight tolerances; and what would be the maximum reasonable vane-to-vane voltage gradient for the beam-channel design.

Our method for the analysis of specific RFQ accelerator designs is the PARMTEQ beam-dynamics simulation program described above. We now discuss the procedures we have developed for the synthesis of RFQ designs. We will describe in more detail the required functions of a typical RFQ. These functions can be divided into four parts, as shown in Fig. 2. The first part is the radial matching of the $d c$ beam from the injector to the input of the RFQ. Because the electric fields of the RFQ vary with time, to obtain a high acceptance of the input beam, we introduce the radial-focusing forces gradually enough to allow the beam to adapt itself to the RFQ time-varying focusing fields. We call this process adiabatic radial matching. The RFQ pole tips start with a large radius and, over a length of about $5 \mathrm{~cm}$, the radii of the poles are reduced to a much smaller value. We assume that the intervane voltage does not vary with $z$, so that reducing the pole-tip radius increases the focusing forces to their full strength at the end of the radial-matching section. From the end of the radial-matching section, we usually hold the average radius of the pole tips constant. This means that the time-varying electric-focusing amplitudes are constant for all values of $z$. This feature, together with the velocity independence of the electric force, means that the RFQ has excellent radial-focusing characteristics.

The second part is called the shaper. In the shaper we begin to introduce the longitudinal field very gradually and, begirning with a synchronous phase of $-90^{\circ}$, we begin to bunch the beam. By the end of the shaper, we have formed a phase-stable bucket in which we have a specified distribution of particles. The third part is the gentle buncher that continues the bunching process in a sufficiently gradual manner so that in the presence of space-charge forces only a minimal radial emittance growth occurs. The gentle buncher makes the transition from a condition of low-acceleration efficiency to a condition of high-acceleration efficiency, with the synchronous

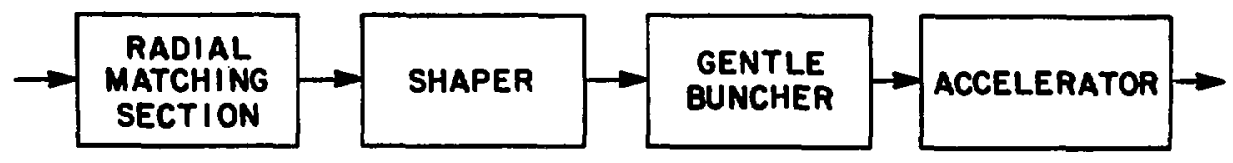

Fig. 2.

RFQ functional b lock diagram. 
phase having its final value. The fourth part is the acceleraior, in which acceleration of the ions is continued at a constant synchronous phase and high efficiency.

In addition to the design requirements discussed above, we impose additionai constraints that are obtained from space-charge scaling laws. These considerations are discussed in the section on heavy ion fusion.

other theoretical studies increased understanding of the RFQ as an element in an electronic circuit. SUPERFISH was used extensively to investigate field distributions, power requirements, and resonant frequencies. It also was used to evaluate mechanical tolerances and the effect of $f a b r i c a t i o n$ errors on RFQ performance.

A mathematical model of the cavity resonator was based on the interpretation that the structure comprised four weakly coupled oscillators. It incorporated a chain-matrix analysis for the RFQ and the coaxial-power manifold. These techniques proved very useful in understanding observations from the laboratory.

Another software tool was developed--a code that manipulated beam-dynamics results and produced a data set for geometrically describing the RFQ vanes. This data set was a complete 30 description that directed vane fabrication on a numerically coritrolled (NC) three-axis vertical mill. The code made allowance for correcting tool paths for various cutter sizes, and for fabricating a similar vane design using different sized vane blanks. The work also required a complete reprogramming of the milling machine microprocessor, modification of the mill controller circuitry, modification of a cassette terminal, and transmittal and storage of the data from CCF files to cassette tapes. The system proved to be an efficient method for fabricating the extremely complex vane geometry, with tolerances held to about \pm 0.0005 in.

2. RFQ Proof-of-Principle (POP) Beam-Dynamics Design. It was highly desirable to provide an early experimental verification of the RFQ principle as well as our ability to design working RFQ resonators. A plan was devised to test an RFQ resonator by injecting it with $0.1-\mathrm{MeV}$ protons from an existing injector, and to power the resonator with an existing $425-\mathrm{MHz} k \mathrm{k}$ ystron. In addition to these constraints, the RFQ vane length was dictated to be $1.1 \mathrm{~m}$ by an existing tank to which the klystron waveguide was attached. With these restrictions, we made: a beam-dynamics design that emphasized proper radial matching and bunching rather than maximum energy gain. The aperture of this 
RFQ was small, as determined by the rather high frequency and by the use of a conservative peak surface field of $30 \mathrm{MV} / \mathrm{m}$. The aperture diameter ranged from $0.4 \mathrm{~cm}$ at the shaper input to $0.26 \mathrm{~cm}$ at the accelerator output. With the restrictions imposed by existing equipment, the output energy was limited to $0.64 \mathrm{MeV}$. The nominal input beam current was considered to be $15 \mathrm{~mA}$, which gave a predicted output current of $14.4 \mathrm{~mA}$ and a small radial emittance growth. However, with an input current of $30 \mathrm{~mA}$ the output current was predicted to be 25.9 $\mathrm{mA}$ and the radial emittance growth was still expected to be small. This design is discussed in greater detail in other papers. ${ }^{5,6}$ The beam-dynamics design was completed in August 1979 and we anticipate a full-scale test with beam early in 1980 .

Driving the RFQ with $r f$ power was another field of accomplishment. Early in the year, the necessity for manifolded power coupling was recognized. This resulted in the idea for a coaxial cavity that would distribute rf drive symmetrically to the cavity. Two schemes were tested for coupling power from the coaxial cavity into the RFQ. Resonant coupling loops were discarded because of tuning difficulties. The method finally chosen was that of providing a series of nonresonant coupling slots in the RFQ cavity wall. This technique behaved as analysis predicted and was easy to incorporate into the cavity design.

There were several other aspects developed in understanding the RFQ as an element in an rf circuit. Capacitive tuners between the coaxial tank and the RFQ were shown to be effective for shifting the power distribution inside the coaxial resonator. A procedure was developed for al tering field distributions inside the RFQ by using capacitive end tuners.

The POP test beam-line layout and diagnostics design were also completed in 1979. The beam-line layout resulted from many TRACE runs, constrained to the use of locally available magnets and hardware. Designs were completed for many pieces of diagnostics gear, including beam boxes, stepping motor and pneumatic linear actuators, beam-profile harps, emittance slits and collectors, a beam stop, and a four-jaw collimator assembly.

A great deal of equipment was delivered for the POP test in 1979. Some of it, like the 1500- $\ell / \mathrm{s}$ turbomolecular pump and a new helium leak detector, was purchased. Part of the equipment was to modify existing gear, as for the PIGMI ion-source changes and Instrumentation and Controls (I\&C) and diagnostics wiring. The bulk of POP equipment, however, was fabricated at LASL. The list 
includes beam-diagnostics hardware, beam-line components, magnet-alignment stands, the cavity resonatur, the vanes, and hundreds of small items.

3. RFQ Sparking Test. About midyear, an experiment was defined to test the voltage breakdown properties of a quadrupole resonator. The scope was to determine what level of voltage gradient could be expected to produce arcing between vane tips. The design jobs involved mere for a cavity/vacuum envelope, vanes, a waveguide section, and miscellaneous hardware. The test was done for the three types of vane-surface finish. The results showed greatest stand-off for electropolished vanes; hand-polished and electroplated vanes also were tested. The sparking field for the electropolished set was $47.0 \mathrm{MV} / \mathrm{m}$. These data were used as a design basis for the POP test, where $30 \mathrm{MV} / \mathrm{m}$ was chosen as the operating gradient.

4. Preliminary FMIT RFQ Beam-Dynamics Designs. Most of our effort has been in developing a general understanding of RFQ beam dynamics and in developing procedures to design specific RFQ systems. Part of this work has used the FMIT RFQ as an objective, and this has resulted in several preliminary FMIT designs. These examples show that we can meet the specifications; however, little effort has been spent so far in optimizing specific linac. characteristics. Table I gives the principal RFQ specifications for the FMIT accelerator. Also, there are constraints on the overall length of the RFQ and on conditions related to matching of the RFQ output to the input of the drift tube linac.

TABLE I

RFQ SPECIFICATIONS

Partic le

Frequency

RFQ Input

dc Beam

Energy

Radial Emittance (Area $/ \pi$ )

Normalized Emittance deuterons

$80 \mathrm{MHz}$

$0.10 \mathrm{MeV}$

$10 \mathrm{~cm} \cdot \mathrm{mr} a d$

$0.10 \mathrm{~cm} \cdot \mathrm{mrad}$ 


\section{RFQ Output}

Synchronous Phase

Energy

Radial Emittance

Normalized Emittance

Emittance Growth

Beam Current $-30^{\circ}$

$2.0 \mathrm{MeV}$

$<6.5 \mathrm{~cm} \cdot \mathrm{mrad}$

$<0.30 \mathrm{~cm} \cdot \mathrm{mr}$ ad

$<3.0$

$100 \mathrm{~mA}$

In a typical RFQ design example, we imposed the requirement that the calculated radial and longitudinal beam-current limits be equal in magnitude. In this case, these limiting currents were $210 \mathrm{~mA}$. For a 110-mA input current the output current, as determined by PARMTEQ calculations, was $104.2 \mathrm{~mA}$ or $94.7 \%$ transmission efficiency. The radial emittance growth ratio was 2.5 for the radial phase-space ellipse containing $90 \%$ of the output beam. The rms emittanse-growth ratio was 2.4. All of these results are within the specif ications listed in Table I. We plan to generate a variety of RFQ designs from which the final choice can be made.

\section{Beam Diagnostics}

We will measure particle beam profiles, densities, and transverse emittance by measuring the light emitted from the passing interaction of the beam particles with residual gas atoms in the transport lines.

Primary technical progress for 1979 lay in demonstrating the capabilities and feasibility of the light collection, signal processing, and reconstruction coding. The density distribution of the preprototype injector beam was tomographically reconstructed from the measured profiles taken by a vidicon TV camera, line-scan analyzer, and ADC combination. Horizontal and vertical profiles were digitized and entered into a computer-based reconstruction code based on attaining a maximum entropy solution. The output density distribution was thus obtained in a totally noninterceptive manner.

The absolute light level from the preprototype injector was measured as a guide for extrapolations to the light levels at the FMIT beam energy. The spectrum of the light emission was measured as input data for a study of the physical process of beam interaction with the residual gases. These studies 
have begun and our work is being compared with similar studies by John Fraser at Chalk River.

A low-light-level TV camera with an intensified silicon target (ISIT) was used to demonstrate proof-of-principle by viewing the much reduced intensity of the Los Alamos Meson Physics Facility (LAMPF) accelerator beam.

The reconstruction coding has been set up not only for $x-y$ spatial densities, but also to give a 40 reconstruction of the beam emittance in both transverse planes. The 4D emittance code has been shecked with test cases. Design is now underway to properly space the four stations longitudinally along the beam line to maximize the information from the reconstructed emittance.

In the collaborative effort on the RFQ POP test, a full complement of di. jnostic sensors in modular housings was provided to instrument the PIGMI beam line for the 425-MHz RFQ test program. The added diagnostic boxes and sensors include a full emittance measurement station, view screens and 4-jaw scrapers, wire scanners, harps, current transformers and two versions of energy-spectral detectors.

A laboratory for sensor and electronics development was outfitted and is fully functional. Preliminary versions of a beam-simulating pulser were built, tested, and used. Several models of the sensors for time signals, beamposition monitors, and current transformers were designed, built, and are being tested with the pulser.

Design input was provided to accommodate position monitors in the bore of the drift tubes for the prototype linac. Design and testing is underway for a vacuum feedthrough and associated cable that will be resistant to fast. neutrons up to $10^{22}$ per square centimeter and will have a bandwidth well in to the gigahertz range. Design input to the linac tank design included the spacing for several portholes in the tank to allow optical views of the beam in the gaps between selected drift tubes.

D. rf System

The preliminary design of both the FMIT and prototype rf systems was essentially completed in the past year. 7 FMIT will require fifteen rf amplifier systems and the prototype will require four. Each system must deliver $600 \mathrm{~kW}$ and must be phase controlled to $\pm 1^{\circ}$ and amplitude controlled to $\pm 1 \%$. Each amplifier system includes a low-power amplifier (LPA) system (0- to 100-W output) and a higher power amplifier (HPA) system (0- to 600-kW output). 
A technical and adninistrative specification has been written for the HPA system and procurement negotiations are in progress with Continental Electronics. Design, specification, and procurement of the high-power coaxial transmission system was also initiated.

The EIMAC 8973 power tetrode (formerly $X-2170$ ) was comprehensively tested and found to perform satisfactority at $80 \mathrm{MHz}$ at our required power output. The tube was tested at $600 \mathrm{~kW}$ and $800 \mathrm{~kW}$ and showed no weaknesses. Successful short runs were made at the 1-Mw level.

Breadboard and testing of the phase, amplitude, and frequency control systems, and the low-power rf system have begun.

A wideband varactor phase shifter has been developed that produces a linear phase shift of $400^{\circ}$ over a $40-V$-bias range.

Design and development of a linear $80-\mathrm{MHz}$ phase detector with a $360^{\circ}$ $r$ ange was started. The unit has been breadboarded and testing is in progress. E. FMIT Facility Control System

1. Summary. The year took the Facility Control Sys sm (FCS) from Conceptual System design through Preliminary design; the design has been reviewed by an independent contractor, in addition to the Blue Ribbon Panel, and bid orders for prototype hardware have been placed. 8 The software-system design has been established around the computer vendors' real-time operating system.

2. Control-System Outline. The FCS for FMIT is based upon a dual mainframe computing system at the Main Control level, wich communicates with operator's' consoles and distributed processing devices. The operator's consoles contain display and command hardware necessary to provide for man-machine interactive functions, which "close-the-loop" in any computer-supervisory control system. The distributed processing device is remotely located among accelerator and facility equipment, and contains microcomputers capable of performing the control and data acquisition functions of process input/output hardware. The mainframe computers communicate with the remote microcomputers $v$ ia serial-data communication hardware (and software) in common use in today's data communication technology. Application software will operate under the real-time operating system supplied by the computer vendor, as will remote application software in the distributed microcomputers. Finally, the equipment interface will multiplex control and monitoring signals, maintaining isolation by both optical and electrical means. Marshalling of the instrumentation 
wire-plant will be done by a computer-scheduling code to maintain the detailed documentation necessary.

3. Specific Progress. Following the presentation of our conceptual design report in late 1978, the I\&C team began the time-consuming task of developing both the overall control philosophy for FMIT and the preliminary software design. Review of these documents, along with the interlock philosophy for the facility, took place in December 1979.

We ordered, and received for test and evaluation, a representative sample of the CAMAC process input/output equipment that we propose to use in the distributed portion of the FCS. We call this collection of equipment the Instrumentation Sub-system (ISS).

A competitive tender for the mainframe computer vendor was made, resulting in the selection of Digital Equipment Corporation (DEC). Both prototype accelerator (FPA) and FMIT computers were thus established, along with the type of microcomputer for the ISS (POP-11/60 and LSI-11 for FPA, and POP 11/70 with LSI-11, for FMIT).

To provide support for the beam diagnostic system development, a collection of hardware and processing equipment similar to that proposed for an ISS was assembled. This station now serves as a development tool in the experimental program leading tc appropriate beam-diagnostics tools for FMIT.

By the close of 1979, we had taken delivery on half of the FPA computer system and placed competitive tenders for half of the FCS hardware required for FPA; successful bids will establish the vendors for FMIT and FPA.

F. Drift Tube Linac

The FMIT drift tube linac will take the 100-mA cw beam from $2 \mathrm{MeV}$ to the final energy of $35 \mathrm{MeV}$. An alternate output energy is required for materialsdamage calibration purposes, so the arift tube linac is divided into two tanks at the 20-MeV point. Progress on the numerous component parts is outlined bel ow. 9,10

The mechanical systems were fully conceptualized during 1979 and all those specific to the prototype accelerator have gone through the conceptual design-review process. Some have also gone through the preliminary designreview process and are now in final design or initial procurement stages.

Modeling has proceeded on the linac-tank shell-cooling system, the drifttube stem support, special vacuum seals, the drift-tube stem-choke joint, 
and the rf model of the prototype tarik. These models have provided, or shortly will provide, valuable design confirmation.

A modular approach ${ }^{11}$ and a concern for remote handling is a guiding criteria for the linac and beam-transport system design.

1. Beam Dynamics. A study was made of the effects of the initial quadrupole gradient on the maximum-particle radius occurring in the first 17 cells of the FMIT drift-tube section, including the effects of alignment errors. This study set the optimum initiai quad gradient for the drift-tube section.

A new statistical techrique was developed to analyze the results of the gradient study. Some aspects of the method were presented in a paper at the Linear Accelerator Conference at Montauk, NY in September. ${ }^{12}$

Two versions of the drift-tube section of the FMIT linac were designed during the year: the first version was discussed ir a paner published in the proceedings of the 1979 Particle Accelerator Conference, San Francisco, March 1979.13 The second version, which is also the version we expect to build, incorporates minor changes that result from a better treatment of asymmetries occurring in several cells.

Still not included in the PARMILA code are some asymmetric effects on the transverse beam dynamics that do not change the longitudinal behavior. Some time was spent on this problem during the year, but the work has been temporarily set aside because of the press of other work.

Considerable attention was given during the year to the theoretical design of the drift tube quadrupoles. A PARMILA study showed that the use of constant-length, constant-strength quads, instead of a more usual tapering iaw, would result in about a $10 \%$ reduction in maximum beam radius, and would simplify manufacture. As a result, the drift-tube linac will use only three quad types, one for each bore-radius value. An optimized design for the 2.5-cm-radius quad was completed. A computer code was written to provide estimates of the leakage flux and the final design takes leakage flux into account.

During the latter part of the year, work began on matching of the RFQ beam to the drift-tube linac. A matching algorithm is being incorporated into the PARMILA code.

2. Linac Tank. The linac tank progress concerns the tank shell and its floor supports. The design effort was almost totally directed to the 
prototype. In 0ctober, a modest level of effort was established to attack the structural analysis for the "FMIT" tank footing.

With the possible exception of diagnostic ports, the prototype tank includes most of the important design features of FMIT. One of the more important is the selection of copper-clad steel over copper plating for the tank shells.

Two means of bonding the copper to the steel were considered: roll bonding and explosive bonding. Investigations of plate manufacturers, and data taken in measuring unbonded areas in roll-bonded plates, indicated that both methods of manufacture are satisfactory. Explosive bonding is done in smaller, thicker sections before rolling to the final dimensions. Consequently, it is somewhat more flexible in the sheet sizes available, and in addition can produce single sheets, whereas roll bonding uses a sandwich technique and produces sheets in pairs. Good bonding and surface finish is available with both techniques.

Other fabrication standards chosen for the tank involve use of welded end walls as opposed to demountable walls using rf seals. Because manned s y into the tanks is prohibited, welded joints in regions of high rf currents, as at the end-wall to tank-shell junctions, will give the best rf service and structural rigidity.

A machined spanner-hatch flange was selected for the tank because it has the adequate strength and stiffness. The flange supports the spanner-hatch cover that seals the girder slot. It must carry large stresses, because it bears against the hatch cover when the tank is evacuated. Concrete piers were selected to support the tank because of their thermal stability, low sensitivity to vibrational excitation, and low cost.

Longitudinally channeled flood cocling was selected for the tank because of the ease with which the longitudinally arrayed tank components (post couplers, vacuum ports, rf drive lines, spanner-hatch flange) can be accommodated. A welded steel shell serves adequately and is simple to install.

Choices for the tank support and alignment mechanisms were narrowed down to roller bearings or a combination of hydrostatic supports and mechanical elevators. The latter system was chosen because of the light adjustment forces and the facility for adjusting the tank from a single location. 
The prototype tank seals will be elastomer in all cases, except where rf surfaces must be joined. For this one case, the spanner-hatch cover for the drift tubes, a metallic 0-ring with an elastomer backup will be used.

3. Girders. The drift tubes will be mounted in a girder system ${ }^{14}$ to facilitate alignment and handling.

The basic configuration of the girders has been developed and preliminary design of the girder for the prototype is underway. The prototype girder contains $15 \mathrm{drift}$ tubes, the same number as in the first FMIT girder.

The girders are to be supported on the tank at all four corners. The support system incorporates a system to permit linear alignment in the vertical and horizontal planes, as well as thermal expansion in the horizontal plane. Positioning and clamping of the drift tubes in the girder are being designed to be smooth and repeatable, bearing in mind the need for the capability to readjust drift tubes in the linac tank without access to the inside of the tank. Alignment capability will be provided linearly in the horizontal and vertical planes and about the vertical axis (drift-tube stem).

Preliminary design of the water and power interfaces is underway. Tests are being conducted to develop a suitable penetration through the top of the drift-tube stems for the power and instrumentation leads. Tests are also being preformed to develop the $r f$ barrier and vacuum seal at the $\mathrm{drift-tube} \mathrm{stem}$ penetration into the tank.

4. Drift Tubes. The major drift tube components for the prototype accelerator are in various stages of final design. Primary emphasis has centered around a feasible and economic design of the water-cooled drift-tube shell. Considerable progress has been made in identification and design of diagnostic end devices. The cooling requirements for the bore tube are being incorporated in to the bore-tube design.

The FMIT drift tubes are in the preliminary design stage.

5. Drift-Tube Quadrupole Magnets. Computer studies showed the concept of constant-length, constant-strength quadrupole-magnet beam focusing to be feasible and acceptable. This concept was then adopted because of the simplifying effect it has on the power supplies, bus system, controls, cooling, girder design, and magnets.

Conceptual design of the low-carbon-steel core and the two-layer coil (including the rad-hard cement insulation system) was done for the three magnet 
types required. Power and cooling-water requirements for all FMIT magnet were determined.

A model of the type $A$ magnet was built to aid in the design, especially the space/clearance considerations. The steel-core shape was then designed and optimized. The coil was designed. Conductor size was determined; the conductor has been procured, fabricated and is on hand. Two test magnets are currently being fabricated.

Beam-steering criteria were determined. A double steering-coil scheme was initially adopted but, later, based on power supply considerations, this was replaced by an individual, opposed steering-coil scheme. A coil model was made and tested to verify that cooling is adequate.

6. Cooling System. The linac-tank-shell cooling systems were simulated on an analog computer and the following conclusions were reached:

- The reference design for the tank temperature-control system will operate stably and will control tank temperature with precision during steady-state operations.

- This same degree of precision should not be expected during major load changes, such as loss of $r f$, because the internal tank gradients are not subject to control. The average tank temperature can, however, be held to within the limits of operation of the rf system in its frequency "search" mode, permitting re-establishment of normal operating conditions within a few minutes during startup.

As a result of this study, it was concluied that the system could be simplified by eliminating the chillers as an element of the control system; this will improve reliability and reduce cost.

A cooling system was defined for the prototype accelerator that is sufficiently prototypical of FMIT to be useful in evaluation of FMIT design and operation.

7. Magnet dc Power Supplies. A 60-kW, 1000-A, 60-V power supply has been specified to supply power to the prototype and FMIT quad magrets. Nine supplies are needed for the prototype and 20 for the FMIT linac. A specification for these supplies was completed. It is planned to use a similar supply for the High-Energy Beam Transport (HEBT) magnets.

The 40-A, 6-V power supplies have been identified for linac-quad horizontal and vertical steering. Two small coils located on opposite pole tips will be excited by each supply for steering. The steering circuits are 
isolated from each other and from the main quad windings. A draft specification has been completed.

A preliminary design of the electrical power, steering, and water connections to the top of the linac drift tubes has been completed.

The design of the 480- $V$ and 208- $V$ ac power distribution for the prototype building MPF-18 has been completed.

8. Vacuum System. High-vacuum pumping of the accelerator structure and beam lines will be done by ion pumps and titanium sublimation puinps. The design for the roughing system includes a Roots-Blower/mechan ical-pump package. The system has been designed to operate at $10^{-6}$ torr, where beam-particle scattering on residual gases is negligible. For minimum maintenance, the FMIT vacuum system is designed for simplicity and reliability in its operation.

The accelerating section of the FMIT facility accelerator consists of two types of structures: the RFQ, which accelerates beam particles from $0.1 \mathrm{MeV}$ up to $2 \mathrm{MeV}$, and a conventional Alvarez drift-tube linear accelerator, which in turn accelerates the beam from $2 \mathrm{MeV}$ to $35 \mathrm{MeV}$.

The RFQ is basically a cylinder with an internal four-vane structure. The present design of the cylinder has six vacuum pumpout ports, with either 12- or 18-in.-diam ports. Each port will have a water-cooled rf baffle. The volume is estimated at $3.2 \mathrm{~m}^{3}$ and the surface area exposed to vacuum is approximately $50 \mathrm{~m}^{2}$. The FMIT RFQ and the prototype RFQ are to be equivalent. Pumping by Roots Blower with a mechanical backing pump, turbomolecular, ion, and titanium sublimation pumps could be used to achieve operating pressures of about $10^{-6}$ torr.

The linac, also a cylindrical structure, is approximately $151 \mathrm{~m}^{3}$ in volume, with $510 \mathrm{~m}^{2}$ of surface area exposed to vacuum. This includes the Energy Dispersion Cavity (EDC). Virtually all accelerator vacuum surfaces are copper. The vacuum-roughing system is designed to use two $1342 \mathrm{cfm}$ Roots Blowers with mechanical backing packages. The intermediate roughing system will incorporate two $1500 \mathrm{l} / \mathrm{s}$ turbomolecular pumps in series with the Roots Blower packages. It is estimated that approximately 2 hours will be required to rough the RFQ and linac to $10^{-3}$ torr. The operating vacuum system will employ five $2000 \mathrm{l} / \mathrm{s}$ ion pumps and $n$ ine titanium sublimation pumps with an estimated pumping speed of $3000 \mathrm{l} / \mathrm{s}$ for a chevron-baffled chamber. Scattering losses of beam particles off background gas is negligible at pressures in the low $10^{-6}$ torr range, for which the vacuum system is designed. 
Net pumping speeds for the RFQ and linac are estimated to be $5000 \ell / \mathrm{s}$ and $51,000 \ell / \mathrm{s}$, respectively. The ion and titanium sublimation pumps will have 18-in. throats. These pumps will be attached to 18-in.-diam elastomersealed gate valves and will be located 18 in. below the peripheral, of the linac, via 18-in.-diam ports. A 95\% optically transparent water-cooled $r f$ grill of modular design will be used above the ion pumps, between the port flange and valve.

The High Energy Beam Transport (HEBT) will use smaller ion and titanium sublimation pumps. The ion pump speed is designed for $500 \mathrm{l} / \mathrm{s}$. The titanium sublimation pumping speed for a 6-in.-diam throat remains undetermined. The ion and titanium sublimation pumps will not be isolated by valves from the small-volume beam lines. This volume is estimated at $0.8 \mathrm{~m}^{3}$, with a surface area of $30 \mathrm{~m}^{2}$. Extra valved-off ports will be used for additional pumping, instrumentation, and roughing of the beam lines with a 100-cfm portable Roots Blower package. The gas type and gas load coming from the targets and beam stop remain undetermined. Differential pumping may be required in the area of the targets and beam stops, if the pressures within the HEBT are not to exceed $10^{-6}$ torr. Fast-acting valves will also be used $(-50 \mathrm{~ms})$, along with pneumatic gate valves, so that beam isolation and line protection $c$ an be used.

The prototype vacuum system will resemble FMIT vacuum system in design and function; in fact, the pump packages and types of pumps are the same. Pump quantities are smaller and the plumbing schemes may differ, but the designs are essentially the same.

We initiated a vacuum component development and testing program, dubbed VACDATA. The functional use of VACDATA is threefold:

- Evaluate titanium sublimation pump performance.

- Develop maintainable vacuum seals and flanges for FMIT.

- Possible fast-closing valve development for FMIT.

The VACDATA laboratory has obtained several pump packages to service experiments. A steel test tank ( 21-in. diam, 6-ft long) will be set up to test the titanium sublimation pumps. The prototype titanium pump chamber (18-in. diam, 25-in. long) is capable of $3000 \ell / \mathrm{s}$ pumping speed with chevron baffle, according to calculations. VACDATA testing will identify true pumping speed of the titanium sublimation pump and will determine backstreaming of titanium. 


\section{Peripherals.}

a. Post Couplers. On our post couplers, the inserted portion (that is, foot and tube), the entry hardware and the bellows-seal hardware are all similar to the post couplers of LAMPF, but are scaled up physically by the ratio of the $r f$ frequencies.

The outboard support structure, drive mechanism, water distribution system, foot-position indicator and clamping method are all different from LAMPF design.

The support structure consists of four round parts threaded into a bottom flange $p l a t e$ and capped by a rectangular flat plate, which completes an open-box framework through which the drive mechanism works. The top plate is removable--the basis of disassembly procedures from the top, which permits replacement of all assembly components, except the center tube, without removing the tube and foot.

The drive mechanism consists of an externally threaded bushing locked to a shaft extension of the central tube, which is threaded through the top support plate and bears upon a shoulder of the central tube extension. Slack is taken up by a compression ring between the shoulder and the bushing. Mechanical advantage is provided by four capstan bars affixed to the drive bushing.

Rotation of the bellows is prevented by entrapment of the upper-bellows clamping plate within the four-corner structure support posts, which linearly guide the bellows as it is driven in and out. The upper plate also provides the rotational vacuum seal between the center tube and atmosphere.

The water distributor is a removable block, keyed into radial position, and locked to the central tube extension by a locking nut operating against a locking ring recessed into the tube extension.

Radial positioning of the foot* is accomplished by rotating the central tube from the water distributor block; when positioned, the central tube is set-screw locked to the upper-bellows guide plate.

A circular indicator plate is mounted atop the bellows guide plate, and a pointer attached to the central tube extension indicates the radial location of the post-coupler foot.

*Note that radial positioning is independent of insertion/retraction; conversely, insertion/retraction can be accmplished with radial position locked. 
Concentric-mated welding täbs are provided to enable an ultimate positional lock and vacuum seal for the post-coupler assembly; however, once this weld is accomplished, none of the vacuum components are accessible until the weld is cut or ground off.

b. rf Slug Tuner. The rf slug tuner is essentially a scaled-up version of the LAMPF design. Modifications to the LAMPF design are those that are due to increased physical size and those based on operating experience at LAMPF.

Brazed-tube water cooling of the slug was chosen: to reduce the mass of water flow required and to assure adequate area cooling with controlled flow pattern, together with a lower mass of water-containment structure.

The 16-in.-diam copper slug is driven from flush with the rf surface (axially) to 6 in. of insertion. The drive mechanism uses the same motor, gears, ball screw, bushing, and sleeve concept as LAMPF used. Two ball bushings, instead of one, provide greater lateral stability for the heavier FMIT slug. The motor drive unit has been reverse mounted to shorten the outboard structure and to simplify the support structure.

Double-seal Helicoflex vacuum seals have been specified for both tank and support-structure flanges.

The welded-in bellows of LAMPF have been replaced by flanged bellows and bolted ring-seal configuration, to facilitate bellows replacement in service. Because the bellows may approach continuous fiexure under feedback control, bellows replacement must be anticipated.

Spring-ring contact between tank entry surface and slug outer surface (per LAMPF) has been eliminated, based on LAMPF experience, which demonstrated that the spring contactor was counterproductive.

c. $r f$ Drive Loop and Window. The $r f$ drive loop/window is designed to be $1 / 8$ wavelength from window to the end of the drive loop. Initial positioning of the loop is one-half inch out from the phantom rf surface (axially). Onehalf inch of adjustment is provided by a spacer ring between the tank adaptor flange and the coaxial transition-section clamping flange. Successive machining cuts of the spacer ring permit adjustment to the limit where the loop surface is flush with the phantom rf surface.

The design specifies Helicoflex (double-hard and soft) vacuum sealing at the coaxial-to-tank flange closure. Two Helicoflex seals are required. It is expected that disposable Viton seals will be substituted during initial positioning trials. 
A spring-ring contact provides $r f$ conduction integrity around the drive-l ine entry port.

The 1/8th-wavelength coaxial section is designed for $35-\Omega$ impedance. The center conductor is water cooled by flood cooling internally, with inlet/outlet conductors through the loop closure bar along one side of the coaxial outer conductor, and is terminated conventionally. The outer conductor is air-cooled copper. Inner-and-outer ring barriers are provided to create a line-of-sight interference path between tank interior and window-a configuration believed to prevent copper deposition on the window surface.

A ceramic window is prescribed. A plastic window may be substituted directly without loss of mechanical integrity of the system. Soft seals are used. The window is in rigid contact on only one exterior circular-surface area; all other forces on the window are exerted through plastic pads that minimize window stress. To a reasonable degree, the window is axially free-floating.

All window 0-ring seals are affixed to the window/bushing assembly prior to installation; that is, out of the radiation area.

The $r f$ path around the window is hard surface-to-hard surface-bolted contact areas for the inner conductor, and clamped compression surfaces for the outer conductor. The conduction path for the inner conductor is on the outer surface; for the outer condurtor it is along the inner surface. A spring ring conductor effects the flange-to-flange path for the outer conductor.

Quick-disconnect flange clamps, operating on tapered flange halfsurfaces, complete the outer conductor assembly operation. Guide pins have been provided to enable lead-in assembly of the outer coaxial line. The innerconductor bullet-nosed adaptor is attached by a single nut to a stud extending through the vindow center bushing. Disassembly and reassembly for window replacement by hands-on and remote techniques have been carefully considered, and compatibility has been conceptually established, although details of the coaxial line slip-fit and transition (to $50 \%$ ) hardware are somewhat dependent upon the designs of the coaxial contractor. Coordination with potential suppliers has been maintained to establish feasibilities.

d. rf Monitor Loop. The monitor loop consists of a "penny"-sized truncated disc, slit to form a loop and brazed to a copper insertion tube at one side of the slit; the other side of the slit is wire conductor connected 
to the center terminal of a vacuum type " $N$ " coaxial connector. The flange of the " $N$ " connector serves as a compression seal against a Helicoflex ring. A flange compression ring screwed into the tank wall is designed to permit angular positioning of the pickup loop. (Soft seals may be used for preliminary determination of optimum angular position.) We decided not to coppersleeve the tank-penetration hole, because the discontinuity to the rf surface is small.

e. Energy Dispersion Cavity (EDC). A two-cell EDC configuration has been developed for FMIT. The rf surface contours, gaps, magnet locations, and rf chamber dimensions were designed using PARMILA and SUPERFISH.

Three chambers are needed: (1) a transition chamber between tank and EDC rf chamber, (2) the EDC $r f$ chamber and (3) a closure chamber. Three model "B" magnets are used--one each in the transition and rf chambers, and one outside the closure chamber, where it becomes the first magnet of the HEBT chain.

To remove the transition chamber magnet, it is necessary to first move it downstream, then extract it vertically through a hatchway. The rf-chamber magnet may be supported and removed as any other drift-tube magnet, but singly, as a unit; thus two "mini-girders" are proposed over the EDC area. The downstream magnet is in atmosphere and will be a component of the first HEBT mudule; this is permitted by use of an inverted domed tank end closure.

Preliminary design of the FMIT EDC has not been started because the EDC is not needed for the prototype and it is not beam-dynamically compatible with the prototype.

At a December meeting on EDC, it was tentatively decided not to adapt the EDC to the prototype FMIT; but instead to consider at a later date the possiblity of constructing the FMIT EDC concurrently with prototype testing, to develop mechanical and rf features. Subsequently, the FMIT EDC would be shipped to Hanford intact.

f. Intertank Spacer (ITS). An ITS concept has existed without change since June 1979. The concept passed Concept Design Review scrutiny without comment. The Blue Ribbon Panel review yielded two major suggestions for the ITS: (1) isolation of the two tanks by means of beam pipe and valves, and (2) increasing the drift space to $2 \beta \lambda(108.6 \mathrm{~cm})$.

A comprehensive study of the implications of the Blue Ribbon Panel recommendations was made. It was concluded that the insert-rotate-socket method of the original concept best fulfilled the requirements for the FMIT ITS. 
The $r f$ surface contours, as negotiated with and approved by the beam dynamicists, permit the saddle-mounted magnets to be rotated $90^{\circ}$ around their support axis for withdrawal and/or insertion within the dimensions of the tank walls on either side.

10. HEBT.

a. Prototype HEBT. In July, it was decided that the prototype would be tested in three phases: (1) the injector; (2) the RFQ; and (3) the linac. Beam parameters at component interfaces were defined and beam measurement requirements were established as a function of component under test. This provided some design criteria for the prototype HEBT.

b. FMIT HEBT. In March 1979, a conceptual design for the FMIT HEBT was presented, using a periodic bending system. The design presented had the last quadrupole positioned $3.5 \mathrm{~m}$ from the target with a 17-in. i.d. aperture. Studies by Phil Meads, of Wm. Brobeck \& Assoc., indicated that the chromatic and geometric aberration in the system was so bad that the $1 \times 3-\mathrm{cm}$ spot-on-target could not be achieved. The decision was made to modify the beamline so that the last quadrupole was $50 \mathrm{~cm}$ from the target with a 5-in. aperture. Studies indicated that beamspill was minimized and the $1 \times 3-\mathrm{cm}$ spot-on-target was achievable. The modified design has been established as the FMIT HEBT design. 15

Based on that design, and other information, Wm. Broback \& Assco. completed the following studie:s:

Beam Transport Line Tolerances (includes misalignment and aberration studies);

Review of the Cost Estimate made by LASL of the FMIT HEBT;

Beam-line Activation in the 35-MeV $\mathrm{D}^{+}$FMIT Facility; and

The Characteristics of the Radiations Generated by $d+D$ Interactions from the Beam Losses that Determine the Shielding and the Activation of the Accelerator Components.

The beam dynamics code TURTLE was modified by S. Cheng and J. Farrell to use a Gaussian input beam and proper energy distribution, based on EDC designs. The overhead suspension and cluster concept was chosen for the FMIT HEBT. 11. Beamstop. During the year, many beamstop concepts were analyzed. The three most promising concepts were a rotating graphite drum, a coppertube array, and a slanting copper plate. The rotating-graphite-drum concept may not be considered, because the large mass of graphite contained within it 
might lead to high out-gassing loads at the temperatures necessary for the drum to radiate heat energy. The copper-tube array, in which the beam strikes a series of staggered tubes with water flowing through them, is now intended for 200-kW service at the RFQ exit. The slanting copper plate, (at an angle to the beam, with water flowing behind it) is intended for 500-kW service at the prototype linac exit. These two concepts will be pursued further, for use on the prototype. The FMIT beamstop tentatively consists of two slanting copper plates in a different configuration from that in the prototype. 
PROTON STORAGE RING

SUMMARY

The PSR is a particle accurmulator that will accept 800-MeV $\mathrm{H}^{-}$particles from LAMPF in one of two modes, accumulate the particles as protons, and deliver the stored beam in short intense bursts to the Weapons Neutron Research facility (WNR) neutron source. In the high-current mode, known as the long-bunch, low-frequency mode, the PSR accepts 750- $\mathrm{s}$ sulses from the linac at $12 \mathrm{~Hz}$, with an average current of $100 \mu \mathrm{A}$, and delivers the particles upon demand to WNR as 270-ns bursts at $12 \mathrm{~Hz}$ with negligible particle loss. The short-bunch high-frequency mode calls for accumulation of individual LAMPF micropulses into six circulating bunches of $1 \times 10^{11}$ particles each, and extraction into WNR at a cycle rate of $120 \mathrm{~Hz}$.

The high currents and short times involved in PSR operation require special development in many areas of accelerator technology. This report relates some of the ideas and accomplishments of our development efforts.

Section I details work on ring active components: the damper, which senses the beam position and by a stripline deflector corrects orbit deviations; the buncher system, which maintains particle-bunch integrity; and pulsed-power development for deflection devices. In Sec. II, passive aspects such as dc magnets and the vacuum system are discussed.

Section III describes the status of the group's experimental physics program; namely, measurement of $\mathrm{H}^{-}$magnetic-field stripping to determine feasibility of the neutral injection scheme; multipactoring measurements, which are to specify the extent of beam-induced electron aval anche; and energy measurements in the WNR transport lines to determine if the LAMPF beam-energy spread and slewing need correction to within the PSR acceptance.

In Section IV, practical calculations are outlined. Among these are calculations of ideal orbits, beam-induced desorption from the vacuum-chamber walls and considerations for survival of a stripper foil under bombardment by the intense PSR beam. 
I. ACTIVE COMPONENT DEVELOPMENT

A. Beam Monitoring and Damping System

During this reporting period, an overall outline of the transverse damping equipment was completed, together with the general system requirements for the beam-orbit monitor. The equipment configuration for the transverse damper consists of four major devices: (1) a beamline pickup with minimal sensitivity to beam-induced multipactor effects, together with associated high-bandwidth cables; (2) a high-speed position-sensing processor, having an output normalized for intensity variations and operable for both short-and long-bunch inputs; (3) a broad bandwidth, 5-kW deflection amplifier and associated preamplifier to rapidly damp transverse oscillations or instabilities; and, (4) a beam-deflector structure capable of responding to nanosecond variations in supplied deflection power.

To date, one of three candidate beam pickup structures, the inductive loop, has been fabricated for electrical and vacuum tests.

The response of this pickup was analyzed ${ }^{16}$ for beam pulses longer than the beam-pipe radius and for a train of short pulses. Another analysis 17 was completed on the reponse of a position monitor as the beam changes position in the $x y$ plane. The simulation of this behavior was done using an unshielded twin-axial cable through the detector, or a coaxial arrangement with a center wire acting as the beam.

The usefulness of beam-induced background gas ionization for measuring the beam profile was evaluated. ${ }^{18}$ The results indicated that spatial resolution of several millimeters is possible with a 35-A circulating beam if a magnetic guide field of about $0.1 \mathrm{~T}$ is applied parallel to the clearing electric field.

The requirements on the high-speed position-sensing processor system were fully detailed ${ }^{19}$ during the year. The first-time machine-tuneup sequence was outlined, resulting in a full description of the monitor system as an aid in commissioning, specifying the locations for the position/intensity sensors, and outlining the general characteristics of the monitor system, colitputer data acquisition, and control system. 
Investigations into the development of a suitable $5-\mathrm{kW}$ broad-band pulse power amplifier began with the fabrication of a 10-tube (tetrodes) distributed amplifier. This device was complemented with a hybrid-driver amplifier consisting of transistor stages at the front end, followed by a medium-power distributed tube amplifier. The two amplifiers were successfuly integrated into a single system during this period and are currently undergoing pulse performance testing.

B. Multipactoring Oriver

The distributed amplifiers described above appear suitable for simulating the short-bunch mode in the multipactoring experiment. The requirements for this experiment are $5-n s, 1000-V$ pulses into $200 \Omega$ at a 17-MHz rep-rate. The pulse width and amplitude have been obtained by driving the distributed amplifier with the planar-triode distributed amplifier. A circuit, utilizing microwave power transistors, has been constructed to drive the planar-triode circuit. This circuit requires $5-V$ pulses, which are available from commercial pulsers. This chain of amplifiers has performed satisfactorily at low reprates. Load resistors, capable of handling the 17-MHz rep-rate, are being fabricated.

C. The 2.8-MHz Buncher System

Fundamental-mode bunching of the $800-\mathrm{MeV}$ protons in the PSR will be accomplished by a resonant rf cavity. This cavity will be ferrite loaded because of straight-section length limitations, and will also require capacitive loading of the cavity gap. Calculations indicate a need for $20-k V$ peak $r f$ voltage at the gap for complete bunching before the beam intensity causes orbit instabilities.

Brookhaven Alternating Gradient Synchrotron (AGS) Ferrite is now available. In original condition, the AGS material has certain drawbacks. In particular, the permeability is too high, dictating very high field intensity, with attendant high power losses. However, if the ferrite were air-gapped, both the initial permeability and the field strength would be effectively reduced.

A short program was written to analyze these various parameters, resulting in a $210-\mathrm{cm}$ total ferrite length, allowing about $70-80 \mathrm{~cm}$ of cavity nardware, cooling, and gap space. This is tight, but manageable. With this much ferrite in the field, the effective $\mu$ (or permeability) is only about 6 . A 14-cm air gap is required, almost one full radian of the toroid. The overall 
result is a low B field of just over $70 \mathrm{G}$, and total power consumption of $25 \mathrm{~kW}$. Heat dissipation of $240 \mathrm{~W}$ per ring should pose no significant problem. An $r f$ power figure of $25 \mathrm{~kW} \mathrm{cW}$ is quite manageable.

Final prototype design is now in process on the power amplifier. A Collins 205-G, 20-kW transmitter has been modified for service at $2.8 \mathrm{MHz}$. The original class of operation has been shifted to yield 33-kW output power. Final power and modulation purity tests are pending receipt of some coaxial and $r f$ capacitor hardware. This amplifier is of ... ntional design, using two RCA $6166 \mathrm{As}$ in parallel. The $6166 \mathrm{~A}$ is an air-c ued power tetrode. Air cooling is possibly less complicated than water cooling, but far noisier and bulkier mechanically, so we may use some water-cooled tubes as replacements.

R\&D efforts on power amplifiers are also progressing on impedanceclamping techniques to reduce the deleterious effects of beam-induced cavity response back on the beam. One technique is to clamp the cavity impedance by driving it with a very low impedance, broad-band power amplifier. The cathodefollower design (common anode) offers this possibility. T. Hardek and W. Chyna at Argonne National Laboratory have investigated this possibility. ${ }^{20}$ We have obtained some feed-back amplifier modules from LASL's Controlled Thermonuclear Research (CTR) Division that contain Machlett 8618 triodes (2 each). Using background from an on-site inspection of Hardek's cathode follower in March 1979 (at Argonne), a module will be modified to perform as a commonanode amplifier. Development will proceed with full mock up of cavity and line pulser to simulate beam current.

A second and more complex approach was described (IEEE Trans on NUCl Sci, Vol. NS-24, No. 3, June 1977, "Compensation of Beam Loading in the ISR rf Cavities", H. Frischholz and W. Schnell, CERN) wherein a beam-current monitor profile is inverted, delayed, amplified, and presented across the cavity gap. This technique essentially places a wall mirror current, with exactly similar modes, at the gap discontinuity, so that the beam sees a zero wall impedance at the gap. Implementation of the wideband high-power design necessary to accomplish this task has begun, using the old AGS amplifier chassis.

The present design uses a pair of $5000-W$ plate-dissipation triodes (3CW5000F3). The first goal is to test the feasibility of a "white follower" design. This is a class $B$ or $A B$ amplifier with single-ended output capability. Normal, single-ended, amplifier design would yield accive impedance control of the driven load during only one-half cycle. Figure 3 shows an idealized 


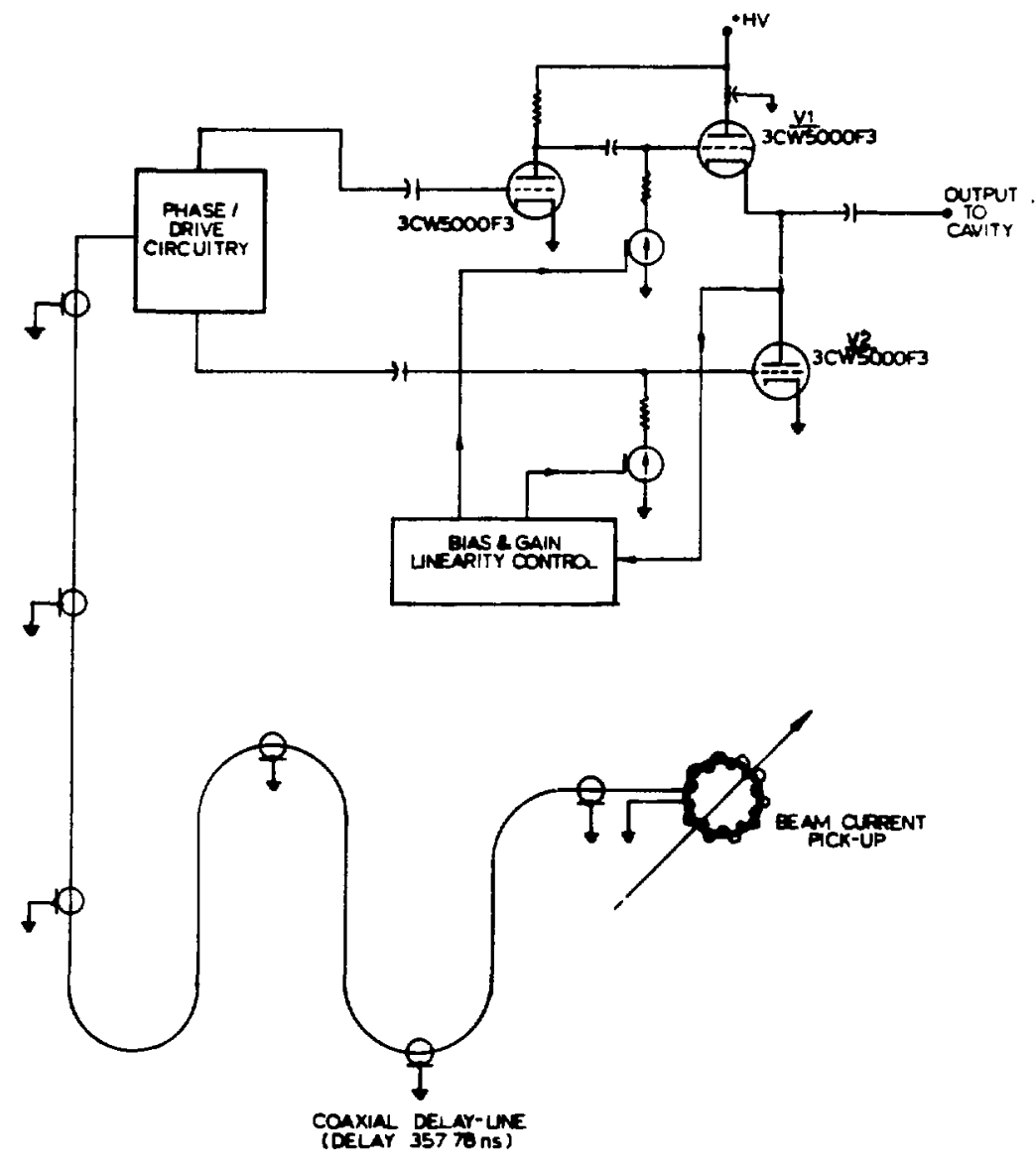

Fig. 3 .

H. F. Buncher - Z Clamp Class "B" Amplifier.

schematic of the white follower. Each half cycle is either current sunk or sourced $v$ ia $v_{1}$ or $v_{2}$, depending on input signal direction.

D. Cavity Gap-Shorting Switch

Design work is complete (short of detailing), on the gap-shorting system. The switch is a sliding sleeve used also as a pump-box sleeve. It is manipulated externally (outside the vacuum system) through bellows by a small dc motor/controller system. This technique makes use of already-committed pumpbox room in a manner that will not require more straight-section length. E. High-Frequency Buncher

1. Buncher Study.

a. Fundamental-Mode Beam Loading. We have evaluated 21,22 beam-loading problems associated with the requirements of the short-bunch operating mode of the PSR. From these requirements we find an $r f$ voltage of $1.5 \mathrm{MV}$ is needed for 
a 603-MHz system. The vector diagram for the beam-cavity system has been drawn and the Robinson stability has been investigated. When we consider the filling of the $r$ ing we conclude that tuning by frequency modulation of the $r f$ drive is unacceptable because the injector linac runs at a fixed frequency. Fastcavity tuning by a reactive external impedance is therefore essential.

For fixed-frequency, tunable-cavity operation, the voltage vector $v_{c}$ is locked to the generator phase $i_{g}$ by the cavity-tuning control loop. If the phase of the injected beam is locked to the generator, it will also be locked to the cavity voltage and hence to the center of the bucket. Because of time lags in beam response, cavity response, and the response of servo loops, it might be better to have a fast servo between the phases of the linac and cavity voltage, this servo acting on a phase shifter in the $r f$ drive. The cavity-tuning servo, because it may have to act on ferrite material, is basically a slower device than a fast-phase shifter at low rf-power level. The stability of these servos can be determined by including their characteristics in the Robinson matrix. 23

Emptying the ring would present different problems because of the discontinuous nature of the process. The largest transient shaking of the beam would be expected when two bunches are left in the $r$ ing and then one of these is instantaneously kicked out. The cavity-tuning servo would tend to reduce the effect of this process, but could be thrown out of lock by the transients. To reduce the transients, we may make rapid jumps in amplitude and phase of the generator current to compensate for the loss in beam current.

Because we do not accelerate the beam during ring filling, no power is required for that purpose. If we keep the cavity matched to the generator under the reactive beam loading, we have no reflected power and constant generator power will ensure constant cavity voltage. An amplitude-control servo is unnecessary for the filling condition.

We require that the phase of the storage $r$ ing be locked to that of the injector. Because the frequencies for each system are derived from a common source, only very slow drifts will have to be corrected.

We do require compensation of the reactive beam loading by adjustment of the cavity tuning. The response must be fast enough to make large adjustments during the ring-filling time, but should cut off before the regime of the fast-phase loop. 
The fast-phase loop is for control of the rigid-band mode of longitudinal multibunch instabilities and to combat interbunch modes; other high-frequency cavities also may be necessary. The response of this ivop shiviiu have a highpass characteristic so that it does not interact with the slower cavity-tuning servo.

To control longitudinal instabilities, we may require a very fast phase control to combat rigid-bunch modes of longitudinal oscillation. This loop would probably compete with the tuning servo, particularly if applied to the main rf system.

During the steady-state condition, with the ring filled, the tuning servo is left on because it will be needed during extraction, and the fast phase loop for control of longitudinal instabilities also is left on.

During extraction, the two previously mentioned servos are again kept running and we compensate for the sudden loss of circulating charge by sharp jumps in amplitude and phase of the generator current. An additional amplitude-control servo also will be required to reduce the input power back to its normal level before the next bunch is extracted.

The rf beam control during extraction is the most difficult task of the servo loops, because an additional amplitude-control loop is required and because the system has to manage large transients.

The phase jumps may se made by ferrite, varactor diode, or PIN diode devices. They must be faster than the cavity-filling time $\tau_{f i l l}=1 / \alpha$ $\approx 10 \mu \mathrm{s})$, and they would be preprogrammed and calculated by the control computer, according to the beam current.

The amplitude jumps could be accomplished by fast-switched attenuators (hybrid junctions and varactor diodes) at the level of the driver signal, or by plate modulation at the driver. The amplitude jumps also must be faster than the cavity risetime and preprogrammed, as the phase jumps are.

b. Stability Simulation. It is possible to find the stability and small-signal transient response of servo systems, including the beam-cavity system if we make the same assumptions that we used in the Robinson stability analysis; that is, we assume that (a) only the zero mode of the rigid dipole motion of the bunches is considered, and (b) only the fundamental frequency mode of the cavity interacts with the beam. A rigid bunch, or macroparticle, is simulated as a delta function of charge. It gains energy according to the voltage already present in the cavity (for large charge bunches we average 
the voltage at entry and exit). The charge bunch induces a voltage in the cavity by charging the capacitor. This induced voltage is added to the value of induced voltage already in the cavity. The induced voltage decays as $\exp \left(-a+i \omega_{0} t\right)$. By using a rotating coordinate system, we remove the $\exp (i \omega t)$ dependence, the induced voltage decaying now as exp $(-\alpha+i \Delta \omega t)$. The voltage in the cavity that is due to the generator at exp (iwt) remains constant if the generator is not programmed.

At the next transit of the bunch (or the next bunch or next macroparticle), we calculate the time of arrival from the momentum-compaction factor of the lattice. From the time delay (since the last particle transit), we find the induced voltage from its previous value and the cavity decay constant (the generator voltage is constantly being topped up and remains static). We now process this new charge bunch as before.

The storage ring DORIS has achieved high currents at low energy, when operating in the multibunch mode. It would be instructive to compare their reactive beam loading with ours.

High-intensity proton machines usually use lower frequency cavities and longer bunches, but we should look at the beam-loading parameters of the SPS and the Fermilab machine.

DORIS does extract single bunches of positrons when filling PETRA but the problems seem simpler than ours: the intensity is less in each bunch; the ring is being filled continuously, so that the loss of one bunch is only about 1/15th of the total charge; the particles are positrons and quickly forget the shaking that they received.

c. Unacceptable Simulation Results. If the simulations show unacceptable conditions, or if our present system seems unfeasible for other reasons, we shall have to change the rf parameters to make things easier.

The main problems stem from the ratio of beam-induced voltage to cavity voltage. He can therefore increase the cavity voltage or lower the beaminduced voltage to make things easier. Both solutions require an increase in rf power.

The cavity voltage may be increased towards the limiting accelerating gradient. (We must note that transient conditions require higher cavity voltage if not properly controlled by the servos.) Cavity heat-dissipation problems would be more severe with a higher gradient. 
The shunt impedance may be lowered by two methods.

(1) Reduce $R / Q$ We may reduce the length of the cavity. The limit here is again the acceptable accelerating gradient and cavity cooling. This reduction in $R / Q$ would have the advantage of reducing single transit effects (short-wake field instabilities).

(2) Reduce $Q$ We may reduce $Q$ by internal means such as lossy cavity walls; or we could reduce $Q$ externally by coupling power out to a load, using another coupling port. (A variation on this is to overcouple the cavity and soak up the reflected power using a circulator and absorbing load.) The tuning cavity or transmission-line stub necessary for cavity tuning may be used to load the main cavity resistively as well as reactively. A ferrite tuning system may well load the cavity heavily, even when designed for minimum loss.

d. Summary. We have had a first look at the requirements of the highfrequency bunching system for the PSR, and using tentative parameters, have seen what problems might arise. We have so far come up against only one obstacle: we find that tuning of the $\mathrm{rf}$ system by frequency modulation is unacceptable; therefore we must have a fast-tuning system for the cavity. Problems that need to be tackied soon are investigation of the feasibility of the fast-feedback systems required, and possible degradation of the longitudinal emittance caused by transients generated during bunch-by-bunch extraction.

2. Disk-and-Washer (DAW) Structure for High-Frequency Buncher. The design parameters for a DAW structure suitable for this application were derived. ${ }^{24}$ The structure would be 9 cells long, with full-cell terminations. The rf would be coupled in from a tapered waveguide through an iris into one end cell. The other end cell would be a suitable location for a large slug tuner and for a coupling loop, which could be used to feed a tunable reactive load. Higher-order modes can be spoiled by manufacturing the outer wall from stainless steel or by nichrome-plating a mild steel outer cylinder. The resulting loss in $2 \mathrm{~T}^{2}$ for the $\pi / 2 \mathrm{~A}$ mode would be only about $2 \%$. Other means to stop propagation or Q-spoil higher-order modes include rotating adjacent sets of washer stems by $45^{\circ}$, and placing coupling loops between sets of stems to feed resistive loads. 
1. Fast-Kicker Development. A 6-mrad deflection (or "kick") is required to direct the circulating beam past the extraction septum into the extraction channel. This corresponds to an $\int \mathrm{B} \cdot \mathrm{dl}$ of $300 \mathrm{G}$ meters, which has to be turned on at extraction time. If an entire 3-m straight section is available for the kicker magnet, the magnetic field required is $81 \mathrm{G}$. It is desirable to make the length of the kicker as long as possible to minimize the magnetic field eriergy $1 / 2 \int_{\sim}^{B} \cdot \underset{\sim}{H} d^{3} V$, which must be switched on and off.

In the short-bunch mode, the bunches are separated by 60 ns intervals, giving a nominal extraction "window" $<120 \mathrm{~ns}$. In this time, the magnetic field must rise, fill the length of the kicker, remain up while the bunch passes through the kicker, and fall, emptying the kicker channel of all magnetic field. The fall-time requirement $c$ an be relaxed considerably if the bunches circulate at 50-ns intervals, with an additional gap of $60 \mathrm{~ns}$ (a "missing bunch") between the first and last bunches. This gap is desirable because of the difficulty of obtaining good short-fall times in real systems with skineffect-induced dispersion and uncompensated lumped reactances (lumped stray inductances or capacitances that cause departures from the nominal impedance $Z_{0}=(L / C)^{1 / 2}$, where $L$ and $C$ are the transmission-line inductance and capacitance per unit length, respectively).

Several possible extraction kicker-magnet types were considered. Ferrite magnets were studied but were deemed unacceptable because of the vacuum and beam-coupling impedance problems they would produce if placed within the beam pipe, and because of the difficulty of achieving rapid penetration of the kicking field if they were outside a beam pipe that was an adequate conductor.

A parallel-plate transmission line will be used as the extraction "magnet." For TEM pulses, $B=E / c$, and the Lorentz force can be written as

$F=q(E+v \times B)=q B c(\beta \pm 1)=\left(\begin{array}{l}2.15 \\ 0.17\end{array}\right) F_{M}$

for a charged particle propagating between the plates with velocity BC parallel or antiparallel to the pulse. Thus, the pulse must propagate through the transmission line in the direction opposite to the beam propagation, so that the electric and magnetic forces deflect the beam in the same direction. 
In vacuum $L C=c^{-2}$, so that the characteristic impedance of the transmission line is $Z_{0}=(L / C)^{1 / 2}=L C$. Using as an estimate of the magnetic flux in a unit length of the 1 ine, $b=B d / 2=I L$ (where $d$ is the plate separation and $I$ is the line current), the required voltage can be estimated as $V=I Z_{0}=B c d / 2$. This calculation assumes the plates to be driven push-pull, establishing a virtual ground at the symmetry plane between the plates. For a 3 -in. aperture kicker with a $50 \mathrm{G}$ magnetic field (the electric component of the TEM pulse provides the additional force required), $V= \pm 37.5 \mathrm{kV}$.

The energy required is estimated to be about 2 joules/pulse from $u=2 v^{2} / z_{0} \quad\left(\tau=33 \mathrm{~ns}, z_{0}=50 \Omega\right)$. The peak power is $56 \mathrm{MW}$, the average power is $2 \mathrm{~J} \times 720 \mathrm{~Hz}=1.4 \mathrm{~kW}$; similar numbers obtain for long-pulse operation.

Such peak-power levels are reached by charging a pulse-forming network (PFN) that serves as an energy store and then switching it across the load (the feed to the kicker in this case). Hard tubes are not practical for such service, although two tubes that operate at comparable levels have been built for modulating tokamak neutral-beam injectors.

Mid-plane triggered, gas-filled spark gaps (or variants on that type) give good $r$ isetimes and triggering accuracy, but both lifetime and maximum repetition rate are seriously deficient. Deuterium-filled multistage thyratrons can handle the peak power; but lifetime is marginal, especially if fast risetime pulses are generated. The lifetime in both arc-discharge (for example, spark gap), and glow discharge (for example, thyratron) devices, operated at extreme current-rate-of-rise conditions, is severely degraded by a common mechanism. There is a transient resistive phase at the onset of the discharge, during which ions can be accelerated into the anode. If the discharge current becomes large during the resistive phase, ions accelerated into the anode can cause substantial heating, erosion, or even sputtering onto the insulating surfaces. Early failure follows. Because the short pulse mode requires $6 \times 10^{7}$ discharge/day, switch lifetime is a critical factor.

The PFNs investigated to date are distributed lines in the charge-line Blumlein, and double-Blumlein configurations. Solid dielectric lifetimes are a source of concern, as is the question of stray capacitances that are imperfectly shielded by the braided outer conductors of coaxial cables. 
It is desirable to charge the PFN to a voltage in excess of the dc standoff voltage of the switch that discharges it. This requires that the PFN be charged and discharged in a time sufficiently less than the breakdown time, to keep the rate of untriggered discharges acceptably low. Command-triggered pulse charging in 1-10 $\mathrm{HS}$ is appropriate: shorter charging times cause excessive prepulse on the load and longer times excessively reduce the margin for voltage holdoff. It is desirable to regulate the charge voltage for shot-toshot reproducibility. The most direct way to do this is with a hard-tube modulator, but $10-\mu \mathrm{S}$ charging currents for the long-pulse mode PFN are too high for tubes of reasonable cost and complexity in application. A slowresonant charging (say $100 \mu \mathrm{s}$ ) of an intermediate energy store (capacitor bank or lumped-element PFN) at 5 or $10 \mathrm{kV}$ can be regulated by short-circuiting a secondary winding on the charging choke to turn off the charging diode. The measured charge on the intermediate store can be used to charge the PFN by a transformer and fast switch in 1 to $10 \mu \mathrm{s}$. This approach avoids the reliability problems inherent in the more conventional approach of direct resonant charging, using thyratrons and inverse diodes. Because the PFN capacitance is so small, the conventional approach is susceptible to stray capacitance, which would store an appreciable fraction of the PFN charge.

We have received a pair of EG\&G experimental thyratrons; they will be tested for the PFN switch application. We have constructed a breadboard model of the regulated pulse-charging circuits and have tested it at full repetition rate. Attainable power levels are presently limited by the secondary sidecharging diodes.

A spark gap that has inexpensive electrodes has been designed. Highvelocity turbulent air flow will cool the arc channel and sweep metastable ions from the interelectrode gap. The switch has not been built because of the considerable expense of supporting mechanical equipment (compressors, ballast tank, cooler, blowdown tank). Should the thyratron approach prove untenable, a spark-gap development program will be reconsidered.

The possibility of using a high-power semiconductor magnetic modulator as a source of the kicker pulses also is being studied. These devices use a series of saturating transformers to make short, high-power pulses from a slow, low-voltage pulse train generated by a thyristor switch. Successive stages are coupled by transformers, which act as switching elements when the Deltamax cores saturate. Each stage results in a halving of the pulse width and a 
doubling of the pulse amplitude. Because the semiconductor switch is used in the slow-risetime, low-voltage first stage, it is not unduly stressed. The remaining switching is done by transformers, which are inherently reliable devices. Such pulse generators are used as airborne radar modulators, where their reliability $\left(\sim 10^{4}\right.$ hrs MTBF), ruggedness, and high repetition rate (up to $4000 \mathrm{pps}$ ) are particularly desirable. A contract is being negotiated for a pulse generator suitable for the PSR fast kicker.

A 350-gal. oil tank with a hydraulic platform lift inside was constructed for testing thyratrons and other components to $100 \mathrm{kV}$. The high-voltage charging transformer has been designed, built and tested. The stray capacitance of the secondary winding stores about $8 \%$ of the transformed charge, but this is decoupled from the PFN by the charging diode and is lost to ground through the winding before the PFN is discharged. This transformer was a source of some concern because there was no commercial unit that was suitable, but the matter is now well in hand. EG\&G is manufacturing one of these for us with standard commercial practice (mounting brackets, taped and varnished coils, etc.). Components suitable for use at high peak currents and repetition rates have been identified and ordered. These include mica capacitors with modified current leads and graded diode stacks. At present, circuit proof-of-principle testing is being done, using the full repetition rate with a $10 \%$ duty factor.

2. Switchyard Kicker for Line D. The fast and slow kickers presently used to kick beam to line $D$ will not meet line $D$ requirements when the PSR becomes operational. The present kickers have the following characteristics: 25

\begin{tabular}{lcll} 
& Fast Kicker & & Slow Kicker \\
\cline { 2 - 3 } & $5 \mu \mathrm{S}$ & $4 \mathrm{~ms}$ \\
Pulsewidth & $8 \mathrm{HS}$ & $500 \mu \mathrm{s}$ \\
Rep-rate & $120 \mathrm{~Hz}$ & $12 \mathrm{~Hz}$ max. \\
Power required & $250 \mathrm{~kW}$ & $10 \mathrm{~kW}$
\end{tabular}

The requirements for PSR operation under the two proposed operating modes are

\begin{tabular}{lcc} 
& Short Pulse Mode & $\frac{\text { Long Pulse Mode }}{\text { Pulse width }}$ \\
\cline { 2 - 2 } & $120 \mathrm{Hs}$ & $1 \mathrm{~ms}$ \\
Rep-rate & $120 \mathrm{~Hz}$ max.
\end{tabular}


The $f$ ast kicker that we are using now can probably be modified to produce a 50- $\mathrm{s}$-wide pulse by modifying the PFN and the magnets. If the PFi inductors are increased 100-fold and the magnet turns increased 10-fold, the pulse width should be $\sim 80 \mu \mathrm{s}$, the current will be $600 \mathrm{~A}$ and the risetime $10-20 \mu \mathrm{s}$. The power dissipation will remain at $\sim 250 \mathrm{~kW}$.

Our present slow kicker would have to be extensively modified to produce pulses $1 \mathrm{~ms}$ wide at $40 \mathrm{~Hz}$ into the present slow-kicker magnet. Also, the slow kicker does not recover sufficiently, following a kick, to have a negligible effect on the following macropulse.

It appears feasible to construct a hard-tube kicker that will provide the following characteristics: pulse width, 1 us to $1 \mathrm{~ms}$; max. duty factor, $6 \%$; risetime, $15 \mu \mathrm{s}$. A hard-tube kicker would have several advantages: it would have variable pulse width and could serve for both PSR operating modes; the flatness of the pulse can be enhanced, using feedback techniques; the total power dissipation will be less than with a PFN-type pulser. The disadvantages of the hard-tube pulser are: a more complex circuit, more active devices, and a new design to debug.

AT-3 has four Scyllac feedback amplifier modules on extended loan, from LASL'S CTR Division, that contain power tubes, and some other components usabie in a hard-tube kicker.

Experience with Machlett $8618 \mathrm{~s}$, in hard-tube modulator service at BNL, encourages their use in the hard-tube kicker. The only major problem at this time is the uncertainty of the tubes' capability in providing a duty factor of $6 \%$. The life of the tubes, each providing $150 \mathrm{~A}$ at $25 \mathrm{kV}$, should be between $25-K$ and $30-K$ hours. Voltage holdoff problems $c a n$ be expected, at anode voltages above $35 \mathrm{kV}$, after approximately 10-K hours of tube life.

3. Bump-Magnet Power Supply. We propose to bump the beam closed orbit into a stripping foil during injection, allowing the peak-current density on the foil to be substantially reduced and creating the possibility of having no perturbing fields when the beam current is accumulated to its maximum value. Four magnets are required, series-connected in pairs. A specifically shaped field, as a function of time, must be supplied (possibly of the form $\left.B=B_{\max }\left[1-\left(t / t_{0}\right)^{1 / 2}\right], 0 t t_{0}\right)$. High-power circuits have been designed for ${ }^{26}$ this purpose. 


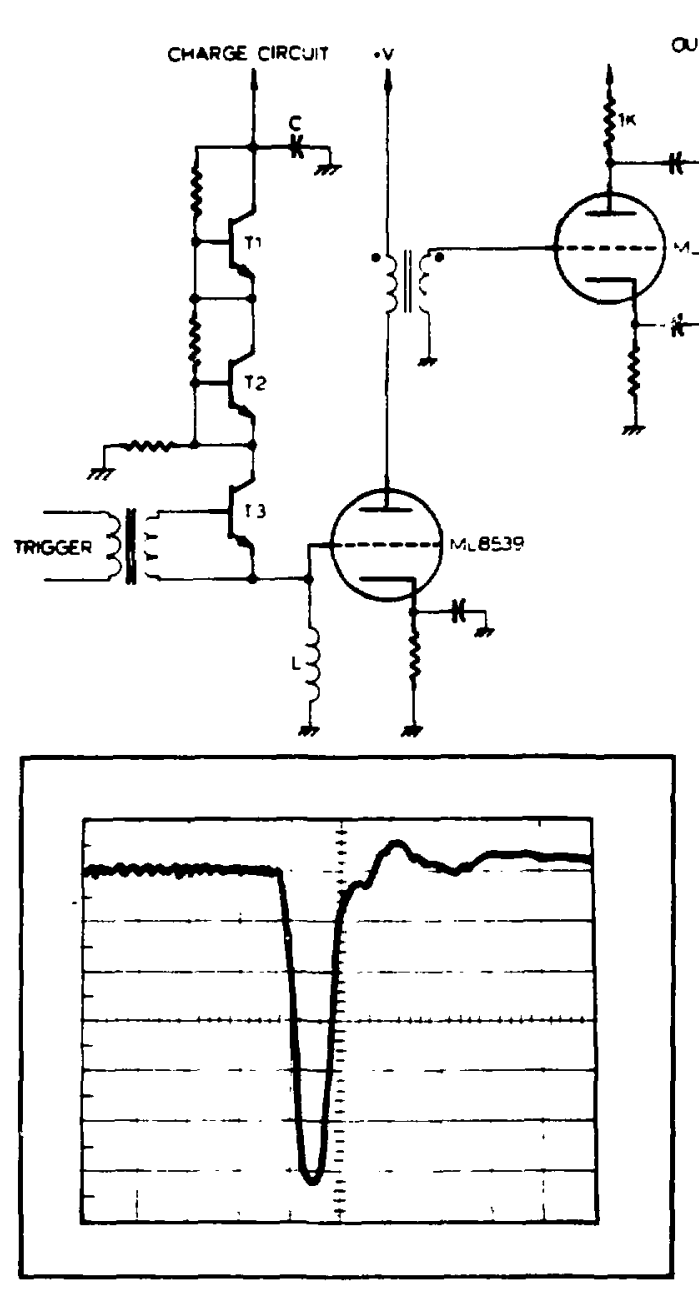

Vertical: 200V/DIV.

Horizontal: 5ns/DIV.

Fig. 4.

Modified avalanced pulser schematic and output waveform.
DOOn

CIRC jis

4. $\mathrm{H}^{+}$Chopper. The $\mathrm{H}^{+}$beam tune in the LAMPF 750-keV injector area has been modified sufficiently so that higher voltage pulses are required from the avalanche pulser to maintain good micropulse-chopping efficiency. The avalanche pulser has been modified to obtain the necessary pulse amplitude and also to shorten the pulse width. Figure 4 shows the modified circuit schematic and the resultant pulse shape. The system is also described in a paper by J. S. Lunsford, G. P. Lawrence, and R. F. Bentley, "Versatile $\mathrm{H}_{1}^{+}$Beam-Chopper System at LAMPF," 1979 Particle Accelerator Conf., March 12-14, 1979. San Francisco, CA.

5. $\mathrm{H}^{-}$Chopper. The $\mathrm{H}^{-}$chopper electronics will be required to operate at much nigher duty factors than the present $\mathrm{H}^{+}$chopper. Also,

it will be desirable to have flexible pulse widths and rep-rates. An amplifier for driving the helical winding is being developed to meet these requirements. A first attempt was based on the Fermi Super Damper distributed amplifier, using 4CW800F tetrodes. Ten of these tubes produced $1000 \mathrm{~V}$ into $100 \%$, with a 2.5-ns risetime. This amplitude may be sufficient, but the risetime is longer than required for good chopping efficiency in the short-pulse mode. The risetime limitation appears to be due to the inherent inductive and capactive parasitics associated with the 4CW800F tubes. 
We have built a second 5-stage distributed amplifier, using ten ML8539 planar triodes in a cascade configuration. The amplfier produces 200-V pulses into $100 \Omega$, with a risetime of less than 1 ns. We will enlarge this amplifier when more tubes become available.

\section{PASSIVE COMPONENTS AND ALIGNMENT}

A. PSR Ring Magnets and Mapping

Trips were taken to PEP, LBL, FNAL, ANL, and BNL to observe magnet fabrication, design, measurement, and powering techniques. We contacted vendors to determine what power supplies of the needed power and regulation are commercially available for powering the ring-dipole magnets. Steel, with proper carbon content for dipole fabrication, was selected and vendors contacted. With the above steel and power-supply choices, dipole design began. We have determined the dipole power system, coil dimensions, conductor profile, cooiling requirements, etc., and pole-tip-shape optimization is in progress. B. Stripper Magnet

A preliminary design for the ring stripper-magnet consists of two dipole sections close to oppositely directed fields. The first section is at high field and the second at low field. The low-field section serves to increase the field gradient between the sections, thus confining the stripping region to as small a distance as possible. The beam-emittance increase caused by stripping then can be limited to a fraction of the original beam emittance. Schwarz-Christofel solutions for the fields of idealized magnets have been worked out. Design problems exist with respect to coil power considerations versus magnet dimensions. Alternative magnet shapes and coil-placement are being explored through the Poisson codes.

C. Vacuum System

The vacuum system activity during the reporting period has concentrated on evaluation of flange-sealing systems amenable to simplified handling and maintenance in the PSR assembly. Emphasis has centered on evaluating commercial metal seal systems that would require minimal flange forces and could provide reliable vacuum sealing during and after in situ bakeout. Tests of candidate commercial seals fulfilling these general requirements revealed that seal reliability was poor, handling requirements were exacting, and seal breakdown under bakeout conditions was prevalent. 
The negative findings from the comercial-seal investigation precipitated a study of a metal-seal concept that had promise of eliminating the ills of axial-force sealing methods. A design concept was developed and systematic testing was initiated on a sealing principle using a soft, deformable copperring seal confined between stainless steel flanges so that axial displacement of the flanges deforms the ring seal into a conical (Belleville) shape. This deformation causes the rectangular cross section of the sealing ring to tilt, allowing the diagonal portion of the ring section to interact with the confining portions of the flange system and producing progressive deformation of the alternate edges of the ring seal as the ring continues to assume a conical shape. The stages of progressive ring-section tilt and radial-force loading are shown schematically in Fig. 5 for a section of the seal and flange system, noted in full scale in Fig. 6 .

We analyzed of the axial and radial forces inherent in this type of Belleville sealing system, using a simplified radial stress calculation based on Hooke's law and incorporated this into a force balance of the ring-flange system. The analysis showed that significant flange-load could be alleviated by introducing a small confinement angle, ${ }^{\theta} \mathrm{F}$, to the flange sealing surfaces, as shown in Fig. 7. This confinement angle serves to tilt the radial-force vectors inward, reducing the initial eccentricity of the force couple, thereby decreasing the magnitude of the radial-force term noted in the simplified force balance. However, subsequent tests have shown that the confinement angle does not materially alter the force-vector direction, but alleviates the axial loads by decreasing the contact area, without jeopardizing the thresholddeformation zone.

Prototype 6-in. flange systems were fabricated and used in a series of seal-evaluation tests to define the reliability of the seal system for longterm and cyclic thermal excursion exposures representative of anticipated activity in the PSR system. The test results showed the flange seal system to have a sealing reliability equal to the best high-vacuum flange system in current use. Bakeout tests showed the same degree of reliability. Repeated thermal cycling, representative of expected bakeout requirements on the storage ring, showed no seal loss. 


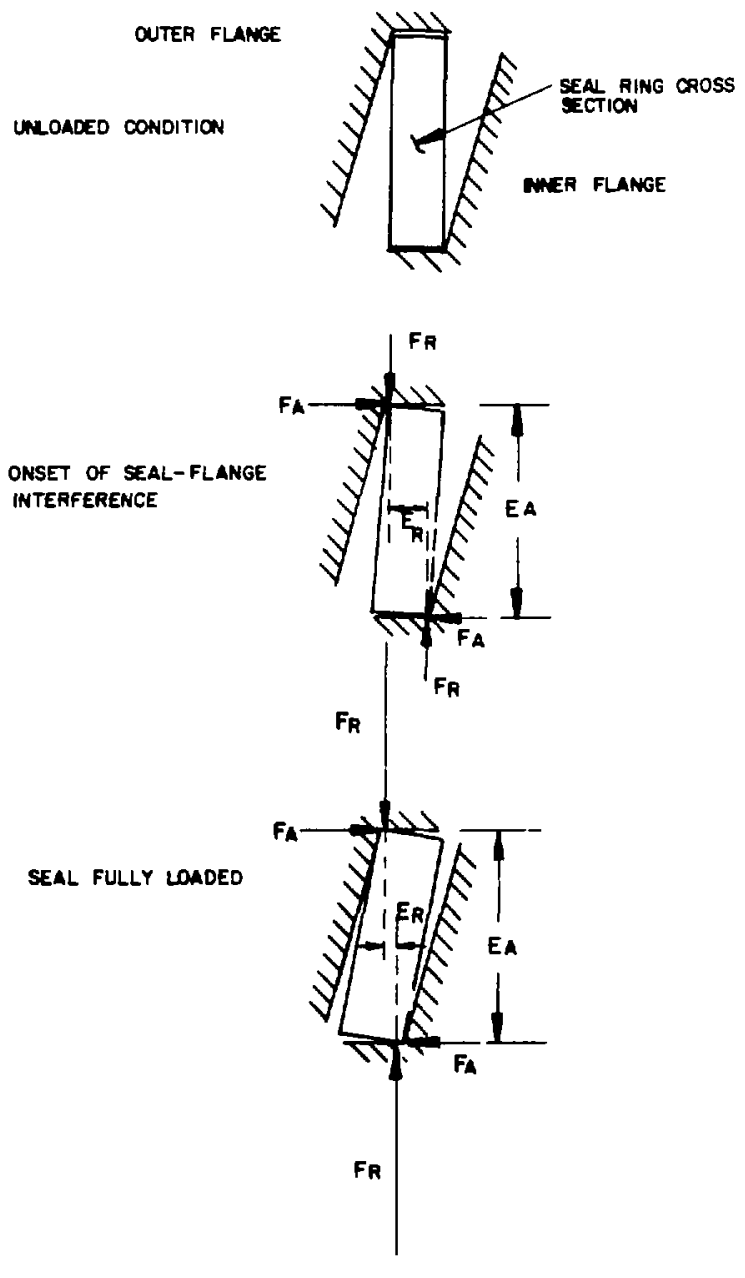

Fig. 5.

Loading of Belleville seal gasket during deformation.

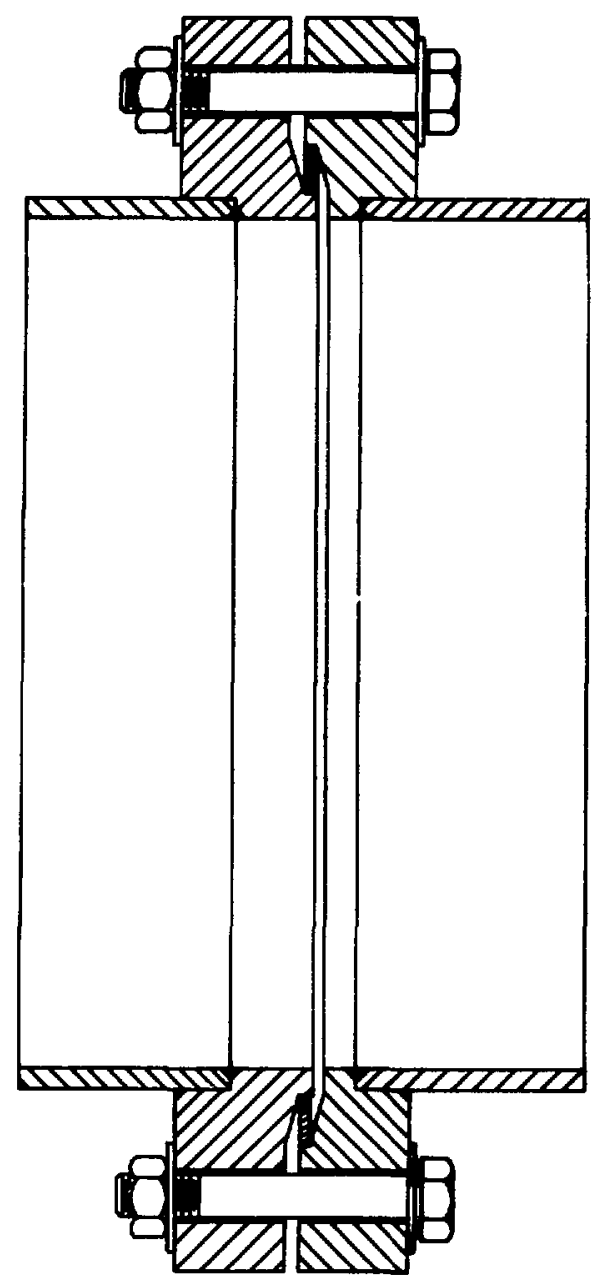

Fig. 6.

Section of Belleville system.

The development of the Belleville flange seal system fulfills the requirements for a simple and reliable flanging arrangement for the storage ring. The low flange forces minimize bolting and clamping requirements, and the flange system can readily be adapted to simplified and remote handing. D. Ring Alignment

The PSR alignment philosophy is constantly being reviewed and revised. We plan to locate eight permanent wall-alignment monuments, two removable floor-alignment monuments around the outside of the ring, and a single reference monument located at the ring center. There will be ten straight sections that comprise the ring. The alignment monuments will be located in 


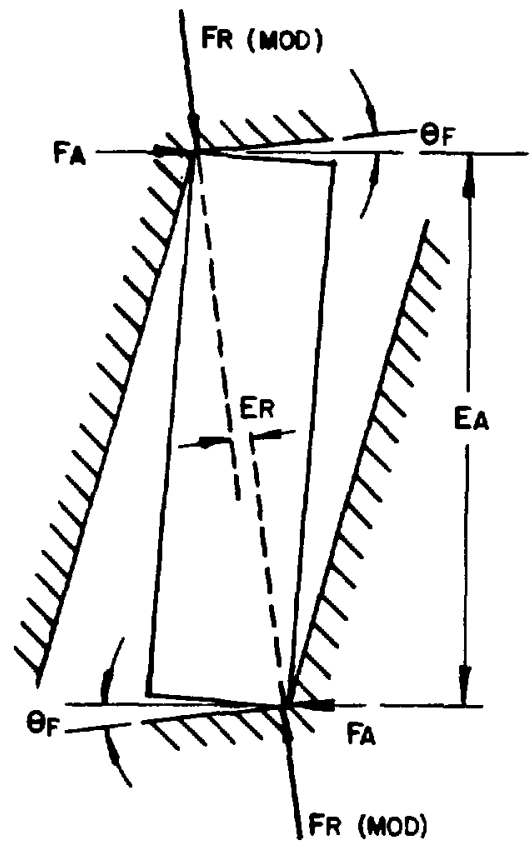

$F_{A}=F(B E L L E V I L L E)+F R(M O D) \frac{E R}{E A}$

Fig. 7.

Force balance on flange sealing surfaces.

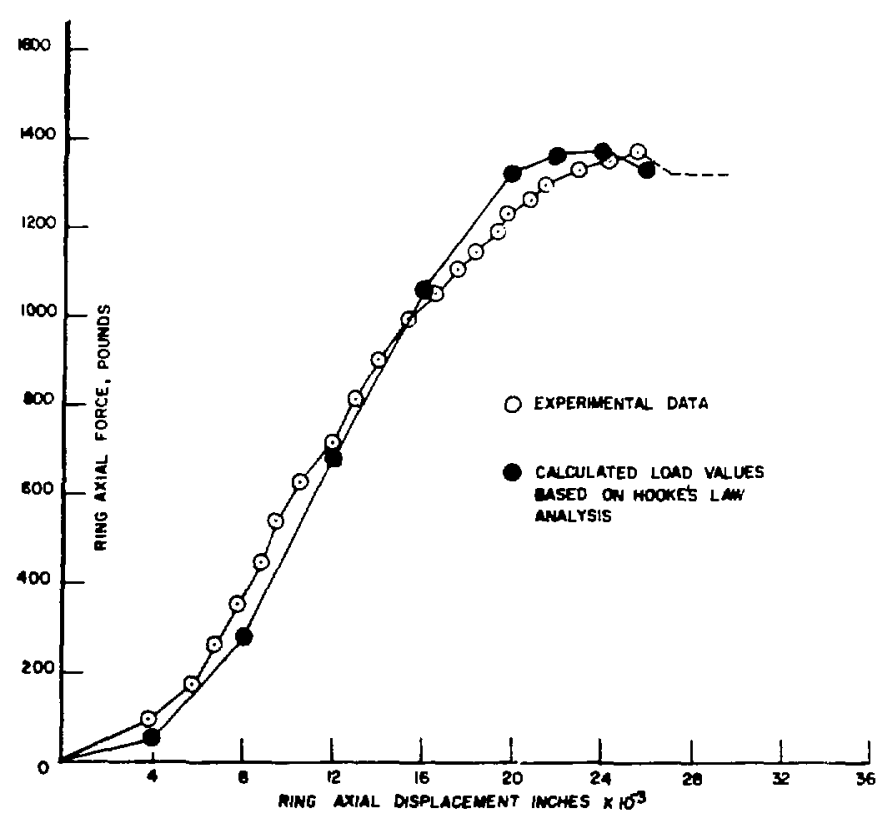

Fig. 8.

Axial load-displacement behavior of Belleville seal.

such a way that a line of sight between two monuments will look directly over the top of a straight section. To accurately locate the alignment monuments around the ring, there will be ten permanent lines of sight: one from each monument to the reference monument through 24-in.-diam sight tubes. There will be an 8-ft-diam tube placed diametrically across the ring, allowing personnel access to the central reference monument; in addition, it will be used as a sight tube to two wall-alignment monuments. The quads, which will be the most accurately aligned magnets in the ring, will be positioned to $\pm 0.13 \mathrm{~mm}( \pm 0.005)$. To obtain this accuracy, the ring-wall monuments must be accurate to \pm 0.001 . Several distance-measuring instruments have been investigated. The most favorable seems to be the CERN Distinvar System, with which it seems possible to measure the distance from the reference monument to the alignment monuments, a distance of $\sim 60 \mathrm{ft}$, to within 0.001 in. It might be that the alignment monuments can be more accurately positioned by taping 
precise lengths and by turning angles, using theodolites. To align each straight section, an optical line of sight will be set up with targets on the components to be aligned. Alternatively, a monofilament line can be drawn taut between two wall monuments, over the staight section, which is a technique used at CERN.

\section{EXPER IMENTAL PHYSICS MEASUREMENTS}

A. Electron Multipactoring

We have designed and built an electron-multipactoring test stand, that doubles as a stand for pumping and beam-induced desorption measurements (Fig. 9). A $100-\mathrm{kHz}$ to $10-\mathrm{MHz}$ oscillator and power amplifier is being built

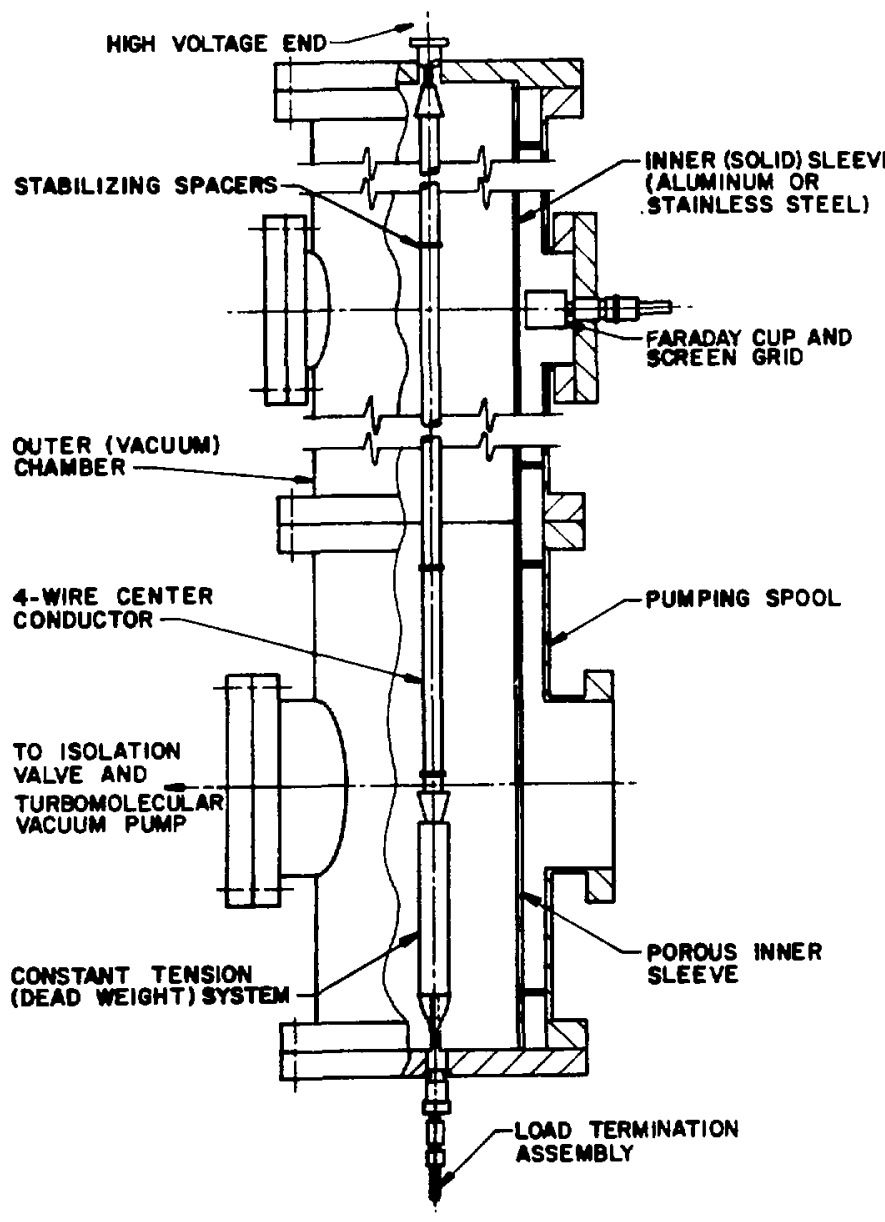

Fig. 9. for simulating the long-bunch PSR mode current pulses. Similarly, a 1-2 ns wide $\sim 1$ kV amplitude pulse generator and amplifier are being built for simulating the short-pulse mode PSR current pulses.

In preliminary tests, we encountered electrically induced mechanical oscillations of the test stand's central wires, and ground loop problems in the electronics. We have eliminated both problems.

B. Stripping Experiment

Knowledge of the rate at which $\mathrm{H}^{-}$ions are stripped in a magnetic field relies on uncertain extrapolations of theoretical and experimental results. Consequently, an experiment is underway at LAMPF to determine the stripping Multipacting test stand (vertical height - rate, as a function of magnetic 3-1/2 meters). 
field strength, to define feasibility and design of the magnetic stripper. The apparatus (shown schematically in Fig. 10) is to be inserted in the EPB line. The EPB line is to be modified by LAMPF to include an additional stripper, which will provide the low-current and small-beam profile necessary for this experiment.

The beam is initially focused with the LAMPF quadrupoles on to the multiwire proportional counter with the stripper magnet unenergized. The stripper magnet consists of Armco iron pole pieces with hyperbolic cross section (designed through the Poisson code to produce a field up to $2 \mathrm{~T}$ ), which increases linearly with distance. At low fields, the stripping rate will increase monotonically with distance through the magnet, and hence with angular deflection. High fields will produce a peaked distribution at the multiwire counter, because of beam depletion over the particle path through the magnet. From the distribution shape and intensity, as compared to the totals count, a stripping rate, as a function of field, can be determined. The low-field magnet near the counter separates the residual $\mathrm{H}^{-}$beam from the stripped neutral distribution. To accurately determine the field at which a particular stripping event occurred, a carbon foil is inserted into the beam at successive positions with in the stripper field. The foil converts most of the as yet unstripped $\mathrm{H}^{-}$particles into neutrals and protons. Hence, the neutral distribution will be attenuated and will exhibit a discontinuity (as recorded by the counter) at a point corresponding to the foil position. The equipment is under construction and the experiment is scheduled to be run during the LAMPF winter cycle.
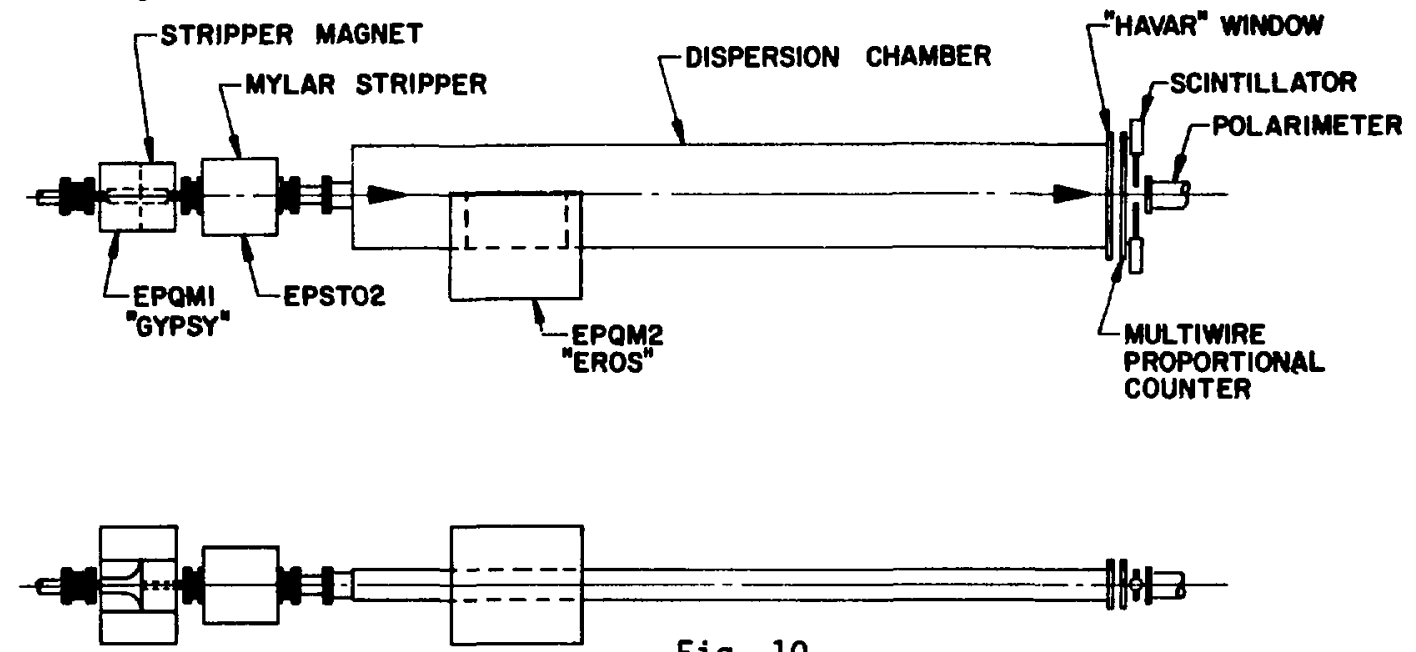

Fig. 10 .

Schematic of $H$ magnetic stripping experiment. 
Energy measurements using time of flight (TOF) between two points along line $D$ have been performed to examine the possibility of using some TOF measurement as a control diagnostic for the PSR, and to examine the energy of the chopped-proton beam presently being delivered to Weapons Neutron Research (WNR). The momentum acceptance of the PSR is $\Delta P / P=10^{-3}$, and although this resolution has not yet been achieved, indications are that a phase-type TOF measurement can optimally attain the necessary accuracy and will be a useful PSR diagnostic. ${ }^{27}$

Using a measurement of phase difference between the signals at two detectors will allow a macropulse-by-macropulse evaluation of the beam energy suitable for a PSR diagnostic. We suggest that two pairs of detectors be used, each with a phase-detection circuit. One pair of detectors would be in the present line $D$, along a straight srition from the bottom of the waterfall to the target-bend magnet, about $150 \mathrm{~m}$ A second pair would have one detector near the waterfall and the other near the PSR injection point. The first pair would be used during tune-up and would serve to determine the energy before injection into the ring; they would then calibrate the second pair of detectors, which would have a fairly large path-length uncertainty, because of the multiple bends, but would yield a precise relative measurement during PSR operation.

Another method of measuring the LAMPF beam energy, using a narrow atomic photodetachment resonance, was tested in the external proton beam line at LAMPF. ${ }^{28}$ Energy and time resolution are adequate to permit the use of this method in studying transient changes in accelerated beam energy.

IV. THEORETICAL AND PRACTICAL CALCULATIONS

A. Orbit Calculations

1. Orbits in the PSR with Ideal Magnets. We are studying singleparticle orbits in the PSR, under the assumption that all the magnets in the lattice are ideal. By "ideal," we mean that fringe fields are ignored; magnets are perfectly powered, positioned and aligned, and median-plane symmetry is achieved. However, we take into account the following nontrivial factors: curvature effects in bend magnets and the nonlinear terms they produce in the equations of motion; nonlinear terms arising in transverse momentum expansions; 
momentum spread in the injected beam; the natural chromaticity of the ring, and the effect of chromaticity-correcting sextupoles.

The main computational tool used in this study is an Adams numerical integration method, which calculates particle orbits to an accuracy in excess of eight significant figures. Closed orbits are found for each value of the total momentum, thus determining the $n$ function exactly, including nonlinear effects, by a Newton search method. The variational equations for motion about these closed orbits are then integrated to determine their Floquet matrices and tunes. In this way the dependence of tune on total momentum (that is, the natural chromaticity), and the effect of chromaticity-correcting sextupoles is easily determined.

To date, we have done these calculations for a ring configuration in which the faces of the dipole-bending magnets are normal to the design orbit (Fig. 11a), we are extending the study to the case where the faces of the bending magnet are parallel. Calculations for this configuration $c$ an be done out by using, as "charts" for numerical integration, two systems of cylindrical coordinates in the two short, straight sections between bends and quadrupoles, and cartesian coordinates elsewhere. This "atlas" of coordinate systems provides a smooth transition between the various $p$ ieces of any given orbit.

2. Transfer Map for Median-Plane Motion in Bending Magnets Having Either Parallel Faces or Normal Entry and Exit. As illustrated in Figs. 1la and 1lb, two types of bending magnet are under consideration for the PSR. To date, most orbit studies have been done for the conceptually and computationally easier case of normal entry. In the case where bending magnets are to be made with solid iron yokes, there seems to be no significant difference in fabrication cost between the two types. However, if the yoke is to be made of laminations, a bending magnet with parallel faces appears to be considerably cheaper. This is because a laminated magnet, with normal entry and exit faces, requires some wedging mechanism to $f$ an the laminations. By contrast, a bending magnet with parallel faces can be obtained simply by sliding laminations.

We are trying to determine whether parallel-face bending magnets have any drawbacks with respect to orbit behavior, using the newly developed method of transfer maps to compare linear motion and first-order aberratioris in the two kinds of bending magnets. 


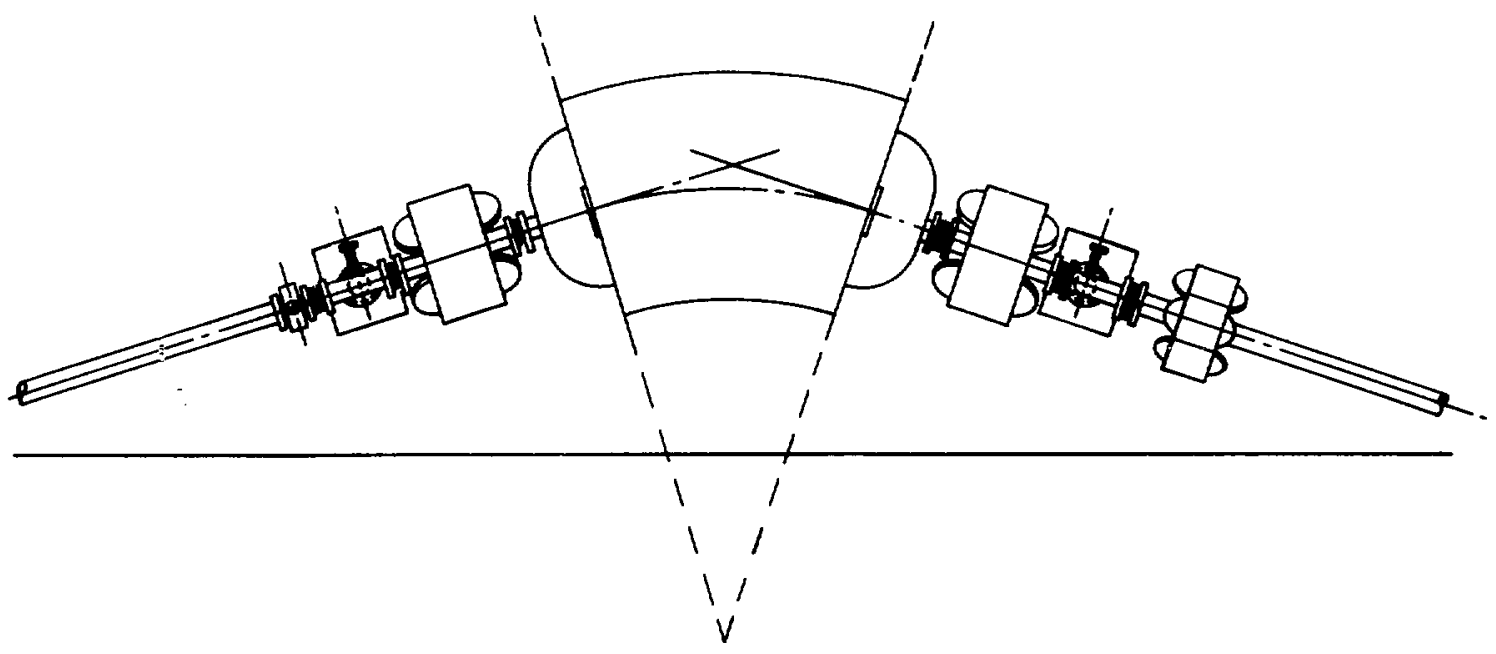

Fig. 11a.

Dipole magnet with beam entry normal to polefaces.

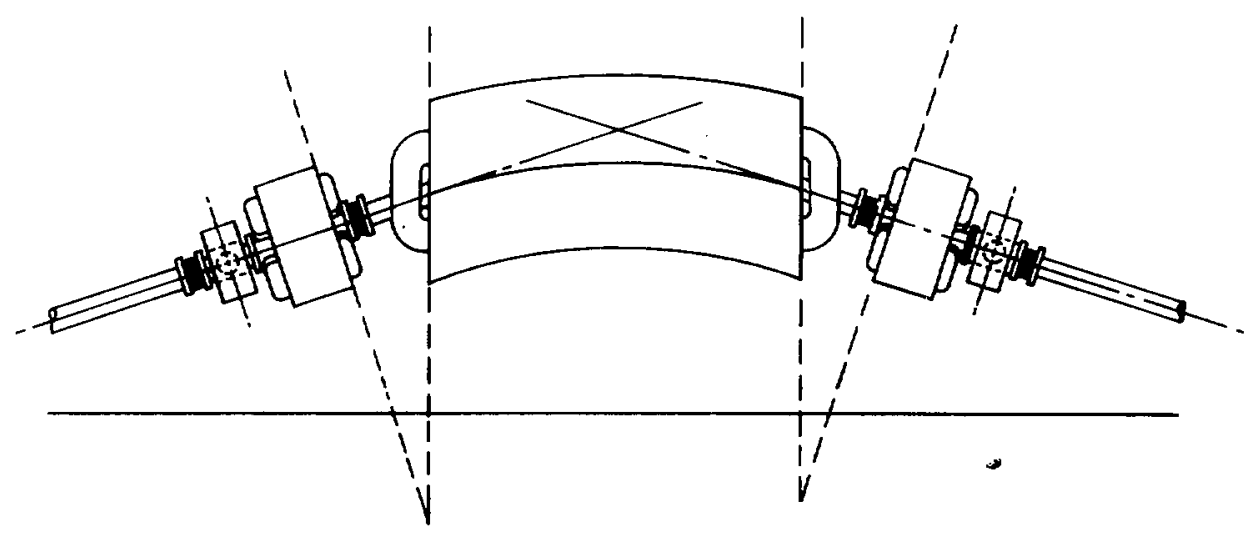

Fig. 11b.

Dipole magnet configuration with parallel faces and non-normal entry.

3. Orbits in the Imperfect PSR. We are considering the use of transfer maps to extend the study to the case where magnets are imperfectly powered, positioned, and aligned, and various magnetic imperfections are present. A knowledge of closed orbits in an imperfect ring would be very helpful in setting power-supply tolerances and positioning and alignment tolerances. A study of the cumulative effect of magnetic imperfections after many turns would be useful in setting field-quality specifications for the bending magnets and quadrupoles. 
The method of transfer maps 29,30 is expected to be much faster than direct numerical integration, but somewhat less accurate. Its accuracy can be checked, however, by comparison of its results with those given by other methods of in various test cases.

4. Beam-Resonance Effects. We have studied beam-resonance effects caused by magnetic multipole field errors and space-charge interaction. Calculations based on the nonlinear resonance theory of Guignard indicate that random magnetic multipole field errors of relative size $10^{-4}$, over a good field region of 5-cm radius, will not cause emittance doubling with in $2 \times 10^{4}$ turns (except for quadrupole errors, which have no effect except at halfinteger tunes). This means that there are no bothersome magnet resonances with in the tune quadrant 2.00 to 2.50 (exclusive of end points) if fields are held to this tolerance, because there are no resonances that result from systematic magnet errors in this tune region.

A computer code for studying the space-charge problem is being developed. To check this code, effects of the space-charge interaction potential term $v x^{2} y^{2}$ ( $x$ and $y$ are transverse coordinates), which appears in the potential of a parabolic transverse-charge distribution, were calculated analytically. This term is important because it is the origin of the only systematic sum resonance $\left(2 v_{x}+2 v_{y}=0\right)$ and difference resonance $\left(2 v_{x}-2 v_{y}=0\right)$ in the tune region. Limiting amplitude increases were calculated analytically for PSR parameters and show that, for the sum resonance, the amplitude can increase only by the factor 1.6. The difference resonance causes exchange of $x$ and $y$ amplitudes, but no observable change in beam size of a round beam.

The computer code integrates particle motion under the full effect of the potential $v x^{2} y^{2}$ (all Fourier harmonics), in contrast to the analytical calculations, which treat only one harmonic at a time. The results of the code and calculations completely agree; furthermore, the code shows the dominance of the difference-resonance behavior, even on a sum-resonance line at $v_{x}=4.0, v_{y}=6.0-a$ reasonable distance from the difference resonance $v_{x}=v_{y}$. This means that the beam will develop equal emittances $\varepsilon$, with beam shape determined by the amplitudes $A_{x}=B_{x} \varepsilon$ and $A_{y}=B_{y} \varepsilon$. This is illustrated by the code, wich takes a rectangular beam into an elliptical beam in 30 turns. 
1. Beam-Induced Desorption Yields. The pressure rise caused by desorption (leading to vacuum-system instability), which the PSR beam may induce in the vacuum system, was calculated ${ }^{31}$. In the PSR, the proton beam will be stored for only a few milliseconds. Various stacking schemes were modeled for average WNR target currents of $100 \mu \mathrm{A}$ and $400 \mu \mathrm{A}$, and the maximum allowed net desorption yields calculated. Continuous PSR operation, using the stacking scheme of main interest (one macropulse stored during $500 \mu \mathrm{s}$ ) presents no problem. Operation using other stacking schemes would require a drastic reduction of the net ion-desorption yield (NDY) or increase of the critical current for the onset of vacuum instability. Methods of achieving this are described below. The emittance growth that is due to scattering from residual gas was also estimated, and no problems are anticipated for $r$ ing vacuums lower than $10^{-5}$ torr with the beam present.

2. Cleaning by Beam Desorption. The net desorption yield of the vacuum chamber may be lowered by using beam-induced desorption itself as a cleaning method. The required dose rate of ions hitting the vacuum chamber walls is obtained (for reasonable cleaning times) if the system pressure is raised $10^{-5}$ torr in the presence of a stored ion beam.

Such cleaning of the walls would require operation of the PSR very near the critical current. This would pose problems, because it is extremely difficult to control the pressure near the critical current, and a small variation in the peak current can make the vacuum unstable. Also, as the net desorption yield decreases because of cleaning, the average circulating current would have to increase to maintain the pressure near $10^{-5}$ torr.

Artificial injection of gas to raise the pressure to the desired level would circumvent the above problems. The choice of injected gas would be determined by the types of vacuum pumps chosen for the ring, their cost and cleaning efficiency: for example, sputter-ion pumps do not pump argon very well, so it would take a long time to reach low pressures after cleaning with this gas. Interlocks between radiation monitors and the extraction kicker would dump the circulating beam as soon as the beam emittance became too large to prevent activation of the vacuum chamber. This method has the following advantages.

a) The NDY is lowered most in places where the beam diameter is smallest (that is, beam potential is high), such as in the dipole magnets and in the 
middle of the straight sections, where pressures are highest and pressure bumps normally appear first.

b) While the PSR is being operated at low levels, the chamber simultaneously could be prepared for high-level operation.

c) If the vacuum chamber became contaminated, it could be cleaned in the proper place by injecting the cleaning gas at that point (turning off a pump near this point to raise the pressure is not recommeded, as this could contaminate the rest of the ring).

d) If a new piece of vacuum chamber were installed, it could be selectively cleaned at a higher rate than the rest of the ring, again by local gas injection.

3. Methods of Increasing Critical Current. The critical current could be raised by increasing the number of pumping stations. Because funding and space in the ring are at a premium, this method is of limited value. The only practical approach would be to connect compact sublimation pumps to the vacuum chambers of the dipole magnets. This involves considerable effort because:

a) Pumps must have large enough apertures to pump effectively.

b) Apertures must to be properly shielded so that the impedance discontinuity presented to the ion beam is small.

c) Sublimators must be properly shielded so that the fields caused by the heater currents do not affect the ion beam

On the whole, the return is small for a large effort, because these pumps would have to have pumping speeds of approximately half that of the main pumping stations to make an appreciable difference.

Because the emittance growth of the stored beam is quite low, even at pressures of $10^{-6}$ torr, it is more important to lower the net desorption yield enough to make the vacuum stable during operation than to achieve a low base pressure. Fortunately, all methods used to decrease the NDY will also lower the base pressure of the system.

Glow-discharge cleaning of the vacuum chamber in an argon atmosphere is most effective. Negative values of NDY (beam pumping) devoid of ion-energy dependence can be obtained. These values of NDY persist even after exposure to air, if the vacuum chamber is baked to $-300^{\circ} \mathrm{C}$ after re-evacuation. Rebaking after exposure to air removes the readsorbed (loosely bound) gas molecules and yields low base pressures, but should have little effect on the NDY because 
bakeouts at as high as $800^{\circ} \mathrm{C}$ never produce negative NDYs nor eliminate the ion-energy dependence. If these readsorbed layers are not driven off by baking, the PSR vacuum system would exhibit base pressures of $\sim 10^{-6}$ to $10^{-7}$ torr, and perhaps high temporary values of NCY, until these adsorbed layers are driven off by pumping and beam-induced desorption.

The time needed to drive off the readsorbed molecules, as well as the temporary value of the NDY, can be studied easily on the multipactoring test stand. The critical currents measured during glow-discharge recleaning of previously cleansed SS tubes exposed to air would yield the needed information.

If the PSR vacuum system becomes accidentally contaminated, the following remedial actions could be taken:

a) Use the stored-ion beam to clean the system, as previously described.

b) Pump high-temperature $\left(-400^{\circ} \mathrm{C}\right)$ air or oxygen through the system to oxidize the contaminant and remove it in gaseous form. The NDY obtained by th is method is low enough for PSR operation in any of the mentioned modes.

4. Conclusions Affecting Vacuum-System Design. We conclude that in situ baking capability is not necessary and that $500-\ell / \mathrm{s}$ diode pumps are more than adequate. Base pressures of $\sim 10^{-6}$ torr are sufficient for operation, as long as the net ion-desorption yield is sufficiently low. Glow-discharge cleaning (in an argon atmosphere) of the vacuum chamber will probably be necessary.

Ports for injecting gas into the system (for cleaning) should be placed in the middle of each straight section and dipole-magnet vacuum chamber. Pumps used for pumpdown must be of a noncontaminating type (preferably not turbomolecular) and should be located in the middle of straight sections, if possible (as far away from dipoles as possible). Perkins-Elmer makes a 12-in.-long, $250 \mathrm{l} / \mathrm{s}$ ULTEK sputter-ion pump with a straight-through port, allowing the passage of an ion beam directly through the pump. A properly designed perforated sleeve inserted into the pump could minimize the impedance discontinuity presented to the PSR beam. An inexpensive pumping station, making optimum use of the available pumping speed (pump closest to vacuum chamber), could result.

If an ion pump is turned on at too high a pressure, a plasma at an elevated potential can be generated by the discharge. This plasma may spread through the vacuum chamber for appreciable distances. A sheath forms between 
the plasma and the conducting-chamber walls. The entire potential difference between the neutral (equipotential) plasma and the wall appears across this sheath. Ions or electrons (depending on polarity of plasma potential) are accelerated to the wall by the resultant electric field. If the ion-current densities and energies are sufficiently large, this system could be used for cleaning the PSR vacuum chamber if suitable plasma "guns" were constructed. A good look at the feasibility might be worthwhile.

C. Stripper-Foil Injection Dynamics

Stripper-foil design is complicated by the large energy deposition of the circulating beam as it passes through the foil during injection. Although the anticipated temperature rise of about $1000^{\circ} \mathrm{C}$ should be tolerated by the foil, thermal cycling may cause rapid foil destruction, from mechanical effects such as shock waves or gross foil vibrations. We are planning experiments to determine the severity of the problem. A spur line exists at the LAMPF injector that can provide about one-half the expected PSR energy deposition in a test foil. Other candidate sources for tests are being explored, including thermal-cycling laser beam and electron gun heating.

Partial alleviation of the thermal problems may be accomplished through adequate injection procedure. Consideration of the PSR phase-space acceptance volume shows that the foil energy deposition can be reduced by approximately a factor of two by appropriately sequenced "bumping" of the circulating beam during injection. This scheme requires four bumper magnets that bump the axially circulating beam a distance from the axis, pass it through the foil at an angle from the axis, and then return it to its former trajectory. Field scheduling for proper filling of beam phase space is under study. If temperatures are kept below damaging values, foil lifetimes of several hours are anticipated. Graphite is the only likely candidate for stripper-foil material. A detailed analysis of foil stripper-design consideration has been completed; ${ }^{32}$ a nominal beam area of $6 \mathrm{~cm}^{2}$ is used for calculations. Foil thickness will be determined by the desired stripping efficiency; beam-emittance spread for adequate stripping efficiencies is found to be small, and foil thickness does not affect temperature or radiation damage considerations. A nominal stripping efficiency of $98 \%$ has been considered for the PSR. 
Recent measurements ${ }^{33}$ for $\mathrm{H}^{-}$stripping by carbon foils have been made at $200 \mathrm{MeV}$ and $\mathrm{c}$ an be extrapolated to $800 \mathrm{MeV}$ with reasonable accuracy. A foil thickness of $300 \mu \mathrm{g} / \mathrm{cm}^{2}$ will provide $99.5 \%$ stripping, whereas $225 \mathrm{\mu g} / \mathrm{cm}^{2}$ are necessary to strip all but $2 \%$ of the beam. The latter thickness corresponds to a dimension of about 1 micron.

Experimental stopping powers correctly give the beam energy loss, but do not completely specify energy deposition in the foil. The injected beam enters as a neutral, and as such interacts differently than does the circulating proton beam. Additionally, some of the foil electrons scattered by the beam have sufficient energy to leave the foil, thus removing energy.

The magnitude of these two effects have been estimated and the net energy deposition calculated. The total energy deposition per injected atom is about $1080 \mathrm{eV}$, whereas on each successive pass the stripped-beam loss is $630 \mathrm{eV}$ per particle.

A further energy-interchange mechanism is due to the energy carried away by electrons, which have been given sufficient energy to leave the foil by collision. This question has not been directly addressed by the literature and is difficult to accurately assess, because of the complexity of electron scattering in a solid. A nonrigorous approach has been adopted to obtain an estimate for this effect. First, the distribution of electron energies is specified. Then, using simplifying assumptions for the electron path, the energies of electrons that leave the foil is estimated. Finally, the relative amount of energy carried away by the exiting electrons is calculated from the energy spectrum. Depending upon the lower limit of energy transfer, a large proportion of energy is contained in the low-energy part of the distribution. The minimum-energy transfer usually used, based on qualitative arguments and agreement with experimental results to calculate stopping powers, is

$E_{\min }=\frac{I^{2}\left(1-\beta^{2}\right)}{2 m c^{2} \beta^{2}}$,

where $I=78 \mathrm{eV}$, the average ionization energy for carbon. 
Electron trajectories can be followed through the foil by neglecting straggling, angular effects and variation in path lengths, caused by scattering along the proton trajectory. Hence, the assumption is that all electrons are created immediately at proton entrance, and travel through the foil for some effective length along the proton trajectory. More realistic assumptions provide cumbersome expressions and are probably not justified on the basis of the approximations previously made.

The obtained contributions to energy deposition in the foil are summarized in Table II.

\section{TABLE II}

FOIL ENERGY DEPOSITION SUMMARY FOR $s=300 \mu \mathrm{g} / \mathrm{cm}^{2}$

\section{Source}

1. Proton-electron scattering

2. Proton-phonon scattering

3. Electron escape

4. Stripped electron

5. Neutral interaction

6. $H^{\circ}$ ionization energy

7. Nuclear reactions

Tota?
Energy deposition in eV/ particle pass

\begin{tabular}{cc}
$\begin{array}{c}\text { Injection } \\
\text { beam }\end{array}$ \\
\hline
\end{tabular}

630

630

neglect

neglect

$-160$

$-160$

450

-(?) neglect

13.6 neglect

neglect

920 neglect

470

The relatively high intensity of the PSR beam raises questions as to foil chermal stability. The probable negative effects of high foil temperature are:

$\mathrm{T}>2500^{\circ} \mathrm{C}$ - foil vaporization

$T>2400^{\circ} \mathrm{C}$ - mechanical failure

$T>1000^{\circ} \mathrm{C}$ - enhanced radiation damage

$T>$ ? - thermal-mechanical damage

Above $2500^{\circ} \mathrm{C}$, the free-evaporation rate of graphite becomes greater than $1 \%$ per second. The mechanical strength, as measured by Young's modulus, and ultimate tensile strength increases with temperature up to $2400^{\circ} \mathrm{C}$, with 
subsequent rapid decrease for higher temperatures. The question of thermal shock, caused by rapid temperature variations and high thermal gradients, is difficult to assess.

In the PSR, the energy delivered to the foil is 2.1 joules/macropulse with an average power of $25.3 \mathrm{~W}$.

Foil temperatures have been estimated through an approximate solution of the equation expressing foil energy balance.

Conduction is a minor heat-loss mechanism and foil temperature is dominated by radiation terms.

The results are utilized in various scenarios where $F$, the "beam-foil interaction parameter", defined in Ref. 32 , is a measure of the energy desposited in the foil.

1. The entire circulating beam passes through the foil, uniformly illuminating an area of $6 \mathrm{~cm}^{2}$. For this case, the beam-foil interaction parameter $F=1 / 6$ giving $T_{\max }=800^{\circ} \mathrm{C}$ and $T_{\text {ave }}=550^{\circ} \mathrm{C}$. The foil may experience thermal shock and damage, because of the large temperature gradient at the beam edge.

2. There is an error in accounting for the energy removed by escaping electrons so that no energy leaves with the electrons. Then $F=0.22$ giving $\mathrm{T}_{\max }=1050^{\circ} \mathrm{C}$ and $\mathrm{T}_{\text {ave }}=600^{\circ} \mathrm{C}$.

3. Possibly, because of a combination of factors, the value of $F$ is double that in (1). Then $\mathrm{T}_{\max }=1500^{\circ} \mathrm{C}$ and $\mathrm{T}_{\text {ave }}=700^{\circ} \mathrm{C}$. These temperatures are probably unsafe.

4. Through injection maneuvers, the beam-foil interaction area is reduced by a factor of three, but the beam intensity remains constant; $F$ then remains constant, so that the temperature distribution is unchanged.

5. Through suitable injection schemes, $F$ is reduced by a factor of two of that in (1). Then $\mathrm{T}_{\max }=490^{\circ} \mathrm{C}$ and $\mathrm{T}_{\text {ave }}=450^{\circ} \mathrm{C}$. This would provide a safe, and probably desirable, temperature.

6. During injection the neutral beam is focused on a 1-m-sq foil area. This alone gives an $F$ of 0.28 . Adding in the circulating beam gives $F=0.45$ with $\mathrm{T}_{\max }=1800^{\circ} \mathrm{C}$ and $\mathrm{T}_{\text {ave }}=800^{\circ} \mathrm{C}$ in the injection area.

7. Spreading out the injection beam over $1 \mathrm{~cm}$ by $1 \mathrm{~mm}$ gives $F=0.2$ with $\mathrm{T}_{\max }=950^{\circ} \mathrm{C}$ and $\mathrm{T}_{\text {ave }}=580^{\circ} \mathrm{C}$ for the injection area. The remainder of the illuminated foil will be at the temperature of (1) for both (6) and (7). 
8. A reduction in $F$ by a factor of two is achieved as in (5). Additionally, the entire foil is deliberately heated (for example, by thermal radiation; to $400^{\circ} \mathrm{C}$. This is approximately equivalent to increasing $F$ by 0.075 , giving $\mathrm{T}_{\max }=800^{\circ} \mathrm{C}$ and $\mathrm{T}_{\mathrm{ave}}=550^{\circ} \mathrm{C}$. The effect is to substantially reduce thermal gradients in the foil.

9. The foil is used in the PSR short-pulse mode with beam-foil interaction conditions as in (1). Using $10 \%$ of the macropulse with a $120-\mathrm{Hz}$ frequency:

(a) The beam is moved off the foil after injection; then the peak temperature is approximately the average, with $F$ the same as in (1). Hence, $\mathrm{T}_{\max }=\mathrm{T}_{\mathrm{ave}}=550^{\circ} \mathrm{C}$.

(b) The beam is kept on the foil between macropulses, during which it is extracted at a linear rate; then $F$ for the average temperature is 2.94; hence $\mathrm{T}_{\max }=\mathrm{T}_{\text {ave }}=1550^{\circ} \mathrm{C}$.

PSR foil conditions have been compared with conditions found in two other laboratories that have similar foil application. The PSR foil is clearly required to withstand harsher thermal conditions than in the ANL or Fermilab applications.

Radiation damage by particles passing through a solid occurs primarily through low-impact parameter scattering. (34) The scattered foil nuclei may be moved directly into interstitial positions (requiring several electron volts of energy); or, for large energy transfers, a cascade is initiated with many interstitial-vacancy pairs created in a locality. The net effect in graphite is to produce interstitials primarily between the hexagonal planes, causing a bulk expansion in the c-direction (perpendicular to the hexagonal planes). The c-expansion is accompanied by a smaller percentage of shrinkage in the a-direction (parallel to the hexagonal planes), because of the tendency of the hexagonal structure to close-on vacancies. This effect, observed to the extent of several per cent with reactor-moderator material, may possibly be directly applied to stripper-foil behavior. It has been observed, mainly in tandem accelerator strippers, that the irradiated portion of the foil increases in thickness by as much as $100 \%$ and tightens in its mount, because of radial shrinkage in the foil plane. A tear then usually begins at the edge of the irradiated area where the strain is highest and progresses across the foil. 
The large increase in thickness observed (as distinguished from the accompanying increase caused by deposition of cracked hydrocarbons under poor vacuum conditions) is surprising and supposes radiation damage beyond the experience of reactor materials. In addition, a predominantly c-orientation would have to be supposed in the foil. This latter possibility is not supported by electron diffraction results, although alternative interpretations, such as very short-range ordering, are possible. A clear explanation of radiation damage in foils, or even of foil morphology, remains to be given.

To alleviate such failures, a simple technique has been effected by preshrinking the foil.35 The foil is mounted on a soft aluminum ring and compressed radially in a collet by about 10 to $15 \%$. This has produced foils with lifetimes increased by a factor of 10 . However, increased beam scattering results from the thickening. Failure apparently occurs when foil slack is taken up by shrinkage.

The effect of temperature variation on radiation damage varies widely with sample structure. Irradiation at room temperature causes simple interstitial defects for low doses. Subsequent annealing at above $200^{\circ} \mathrm{C}$ causes recombination through increased interstitial mobility. High irradiation doses promote more complex defects, such as mobile interstitial clusters and fixed vacancy clusters. Because of their high decomposition energy, sufficiently large clusters are thermally irreversible and tend to grow, causing macroscopic defects.

If the temperature is raised to above about $400^{\circ} \mathrm{C}$ during irradiation, interstitial mobility (and hence recombination) is increased. In addition, defect clusters are broken up by the collisions with the irradiating particles. For bulk samples, the rate of radiation damage (as measured by sample dimensional changes), decreases several-fold for irradiation at temperatures around $500^{\circ} \mathrm{C}$. Deliberate application of this effect is known as radiation annealing. At temperatures approaching $1000^{\circ} \mathrm{C}$ and above, vacancies become mobile and tend to form irreversible clusters, so that damage again increases.

Although corresponding results for foils are qualitative and conflicting, general consensus indicates that constrained foil lifetimes may be increased by a factor of from 2 to 10 by heating to around $500^{\circ} \mathrm{C} .35$ At sufficiently higher temperatures, foil lifetimes again decrease. 
Several techniques have been used to manufacture foils. Of these, preparation by hydrocarbon cracking in a discharge recently has been found to produce foils that are qualitatively better under ion bombardment, in that little shrinkage occurs. 36 The reasons for this are not clear; foil structure is such that the reordering induced by radiation damage produces small dimensional changes. The simplest assumption is that, during manufacture, the high-energy carbon atoms from the discharge are very mobile upon striking the collection substrate, and hence have greater freedom in selection of finalstate ordering. That final state may be then a-oriented, with ordering on a short-range basis. An a-oriented foil would presumably expand along the foil with subsequent slackening and decrease in thickness. This conclusion is partially supported by an observed decrease in foil scattering with time, which has alternatively been attributed to decrease in foil density by recoil carbon atoms leaving the foil. Spectacular lifetime increases have been noted; foils manufactured in this way have been found to last nearly two orders of magnitude longer than conventionally prepared foils.

Using the formalism developed by Moliere 37 for multiple scattering, the half angle, within which $90 \%$ of the particles passing through a $300 \mu \mathrm{g} / \mathrm{cm}^{2}$ foil are scattered, is $0.033 \mathrm{mrad}$. The Moliere theory has been specifically applied to the case of a storage ring by Cooper and Lawrence. 38 Using their formalism, which takes into account a linear rate of injection, and assuming 1400 passes through the foil, the emittance growths are calculated to be

$$
\begin{array}{ll}
\Delta \varepsilon_{X}=0.44 \pi & \mathrm{cm} \cdot \mathrm{mr} \mathrm{ad} \\
\Delta \varepsilon_{y}=0.16 \pi & \mathrm{cm} \cdot \mathrm{mr} \mathrm{ad} .
\end{array}
$$

These figures are to be compared with the approximate calculated acceptance of $3 \pi \mathrm{cm} \cdot \mathrm{mr}$ ad. The emittance growths are proportional to the number of turns during injection, and therefore may be decreased by limiting beam traversals through the foil. In particular, if the beam is allowed to pass through the foil during the short-pulse mode (24 000 turns), emittance growth will approach the beam acceptance. Nuclear reactions in the foil have also been estimated. The $c^{11}$ production represents a radiation hazard, if immediate handling after 
beam shutdown is to be done; however, an 8-h cool-down period will redice the $\mathrm{C}^{11}$ activity to less than a microcurie. The $\mathrm{Be}^{7}$ will most likely be retained in the foil and should be of no concern, unless violent venting or improper handling scatters foil bits into the environinent. Tritium produced in the foil is, however, expected to leave the foil and be adsorbed on the PSR walls and ion pumps. Assuming a $50 \%$ ring duty cycle, the total activation after a year will amount to $68 \mathrm{mCi}$.

It is difficult to predict the effect of high temperatures on foil lifetimes. Uniform heating of an entire foil to above $1000^{\circ} \mathrm{C}$ has not resulted in foil damage. However, the nununiform heating, caused by the PSR beam, will cause severe thermal gradients at the beam edges. A temperature $r$ ise of about $1000^{\circ} \mathrm{C}$ within $500 \mu \mathrm{S}$ periodically will be applied to the foil. It is possible that a mechanical shock wave will then be set up in the foil, because the temperature will rise by about $60^{\circ} \mathrm{C}$ during the time it takes sound to traverse the foil. The foil will probably vibrate at a frequency and amplitude determined by its boundary constraints, as well as temperature-time variation. Additionally, thermal shock is a possible damage mechanism. Bulk graphite is, however, very resistant to thermal shock. In addition, its ultimate strength and elastic modulus increase with temperature and irradiation.

Disregarding the thermal problem, it is possible to estimate foil lifetimes caused by the effects of radiation damage. Tandem accelerator experience 39 has shown that foil lifetime depends on the energy deposited per unit volume. An empirical formula has been developed which, upon ( $r i s k y)$ extrapolation to a beam atomic number of unity and high energies, yields a lifetime

$\tau \sim 3.0 \times 10^{19} \mathrm{E}$ particles $/ \mathrm{cm}^{2}$

with $\mathrm{E}$ the proton energy in MeV. At $800 \mathrm{MeV}$, this gives $\tau=2.4 \times 10^{22}$ particles $/ \mathrm{cm}^{2}$. For the PSR case (scenario 1), $5.6 \times 10^{16} \mathrm{particles} / \mathrm{cm}^{2}-\mathrm{s}$ pass through the foil, implying a lifetime of $120 \mathrm{~h}$. In an application similar to the PSR case, Fermilab estimates (without an actual foil failure) a lifetime of $3.3 \times 10^{20}$ particles $/ \mathrm{cm}^{2}$ at $200 \mathrm{MeV}$. Assuming an inverse proportionality to energy deposition, the estimated PSR lifetime is 2.8 hours. A similar calculation, using Argonne estimates (though for organic foils), yields an 
estimated PSR lifetime of $19.5 \mathrm{~h}$. It is probably best to take the most pessimistic of these figures. If possible an empirical determination of lifetime and thermal effects would be desirable.

Several techniques, which are of empirically verified or hypothetical utility for increasing foil lifetime, are listed here.

1. Decrease flux of particles on the foil by suitable injection schemes.

2. Increase foil-beam intersection area by mechanically moving the foil. This can be done either by mounting the foil on a rotating wheel or by causing the foil to slowly oscillate. It is recommended, however, that the rotating wheel mount not be used, for reasons given below. Alternatively, this can be accomplished by bumping the beam.

3. Preshrink foil, or possibly use an unconstrained foil, if mechanically feasible.

4. Use best methods of foil preparation.

5. Shield foil from electrostatic and pneumatic influences.

6. Reduction of thermal gradients may be of importance in increasing foil lifetime. One method of accomplishing this is to deliberately heat the entire foil area.

Commercial cartridge-type foil changers are available that will hold a large number $(-100)$ of foils. The beam may be moved off the foil between injection pulses, by bumper magnets. The same bumper magnets can be used to ensure proper filling of the circuiating beam-phase volume and to decrease foil temperature through suitable injection procedures. Such injection procedures will be discussed in a separate report, as will bumper-magnet design.

Precautions against foil destruction by air motion during ring pumpdown should be taken. Toward this end, the foil chamber should be isolable from the $r$ ing-vacuum system by a pair of valves, and should be rough pumped arid vented through a needle valve. The foil is also subject to destruction by electrostatic forces. Consequently, placement of insulating surfaces near the foil should be avoided; a grounded copper shield surrounding the foil has been used in other instruments for this purpose. The electrostatic interaction of the beam with the foil should be considered in detail. Symmetrical foil placement, with respect to the beam, is probably of importance in el iminating electrostatic stresses. Electrical conduction between the foil and its holder should be ensured. 
Finally, note that the problems of thermal and radiation damage are somewhat decoupled with respect to catastrophic behavior. A simple experiment with a pulsed electron or proton beam could remove much of the uncertainty as to foil mechanical stability under conditions of high energy deposition.

\section{Beam Transport}

Detailed simulations of the injection and extraction transport lines were completed. 40,41 The novel neutral-beam charge-changing injection technique that substantially simplifies the ring design is described in a paper presented at the 1979 Particle Accelerator Conference. 42 The method consists of converting the $800-\mathrm{MeV} \mathrm{H} \mathrm{H}^{-}$ion beam to a $100 \mathrm{H}^{0}$ beam having an acceptable emittance, and drifting it directly onto the proton closed orbit in the ring. The neutralization is accomplished by electric field dissociation of the $\mathrm{H}^{-}$ions. Liouville's therorem is thus circumvented, and many of the complications of the older, direct $\mathrm{H}^{-}$stripping method are avoided.

\section{E. Computer Codes}

During the first part of the year, considerable effort was expended in getting the $r f$ cavity code, SUPERFISH, which calculates $r f$ fields and associated resonance frequencies for arbitrary geometry, working under the FTN compiler. Previously, we had used local code versions to get some preliminary data. Even though these programs were easy to use, they lacked the flexibility to make minor modifications that our specific problems required. Thus, we converted Holsinger's latest version of SUPERFISH, which has multicell calculations, and made it compatible with the auxiliary programs (AUTOMES and LATTICE) that had been converted to FTN earlier.

SUPERFISH computes the electric field only on axis. To get the eiectric field off axis, we used an old CHAT subprogram named SHY. Changes were made to SHY to make it compatible to the FTN compiler and to plot the electric field for a given $r$ as a function of $z$.

The latest versions of the POISSON codes (AUTOMES, LATTICE, POISSON, PANDIRA, MIRT, TEKPLOT and SUPERFISH) now run under the FTN compiler. We made modifications to facilitate debugging of errors and to make codes flexible to future changes. These modifications include alphabetizing all subroutines, forming a library of common routines used in various codes, including a symbol table with the run files to aid in debugging, summarizing the output files, and adding output files that are readable on the TEKTRONIX or TI terminals. All these codes have been checked, and agree with the CHAT-version results. 
We completed debugging the proton-beam simulation code, PSRS2, added a new force calculation, FORCE2, and optimized the programming in the extensively used subroutines to speed computation time. We also made an LCM (1arge-core extended memory) version that allowed us to increase the numbers of particles to 2000 . 
I. INTRODUCTION

The overall objective of the Los Alamos FEL program is to determine the feasibility of a high-power, high-efficiency, FEL operating at wavelengths in the near-infrared to near-ultraviolet portion of the spectrum. Accordingly, the initial effort in this program was to study the conceptual design of a high-power laser operating at a wavelength of $1 \mu \mathrm{m}$. From this study, the only one of its type of which we are aware, it was determined that such a laser is indeed possible. Moreover, the study served to identify the critical development problems in FEL physics, optics, and accelerator technology. This, in turn, made it possible to outline a program to address these problems. The reasonableness of the program outlined in this fashion is part of the argument for the feasibility of a high-power FEL.

A. High-Power FEL Design

To investigate the feasibility of a high-power FEL, a team of scientists and engineers from several laser and accelerator development groups carried out a conceptual design study for a laser with the parameters wavelength = $1 \mu \mathrm{m}$, average power $=100 \mathrm{~kW}$.

SNGLE PASS

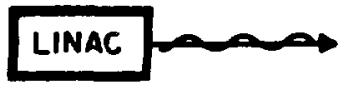

de ReCOVERY

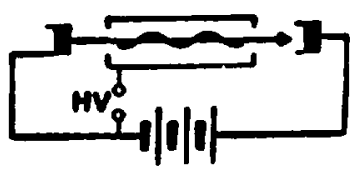

STORAGE RMUS

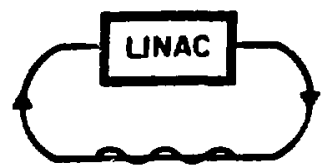

of Recovenr

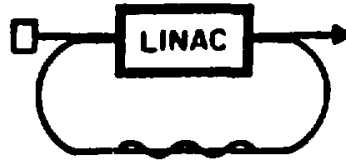

Fig. 12 .

Various approaches have been proposed for achieving high power and high overall efficiency from free-electron lasers. The racetrack rf accelerator/ decelerator energy recovery technique appears to have the best chance of success.

Basically, four types of FEL systems have been proposed. These are illustrated schematically in Fig. 12. In single-pass devices the electron beam is accelerated in a linac or other device and is run through the wiggler where some fraction of the electron beam energy is extracted as laser energy. For wavelengths in the near infrared, $v$ isible, or ultraviolet regions, the extraction efficiency in practical, high-average-power devices is probably limited to less than $10 \%$ even with adiabatic wigglers. This limit is imoosed by the burden on the 
optical components at laser intensities within the cavity required to achieve higher extraction efficiency. The overall efficiency is limited to an even lower value. Because the system cost (for very high average-power systems) depends largely on the input power, this approach is unattractive for short wavelengths despite its simplicity. At longer wavelengths, from the nearinfrared region to the microwave region, low-energy electron beams can be used, and higher extraction efficiencies may be achieved by taking advantage of plasma effects (collective oscillations). 43-46 Linear, single-pass devices may be practical in this long wavelength regime.

To improve the overall efficiency, it is necessary to make use of the energy remaining in the electron beam when it emerges from the wiggler. To recover the energy with a storage ring, the electrons are reaccelerated to make up the energy extracted in the wiggler. The electron beam is then brought around the ring and reinserted into the wiggler. The difficulty with the storage-ring approach is that the momentum distribution in the electron beam is broadened by the FEL. Some electrons are decelerated more than others, depending on their phase with respect to the laser field. This broadening must be damped by synchrotron radiation elsewhere in the recirculating $r$ ing before the beam can be reused in the FEL. Pellegrini has shown that with a uniform wiggler the synchrotron radiation used for damping reduces the overall efficiency to that of a single-pass device. ${ }^{47}$ Special wiggler designs have been suggested to improve the situation, ${ }^{48,49}$ but storage rings are expensive in any case and will probably be interesting only for very short wavelengths that can be achieved with the high energy and low emittance possible with storage rings.

To avoid problems of degradation of the electron-beam quality, it is necessary to recover the electron-beam energy without reusing the electrons themselves. This energy recovery may be accomplished with a dc accelerator by using a back-biased collector to decelerate the electron beam after it traverses the wiggler. 50 Only a relatively small power supply is needed to return the current to the original high potential. Such a system should be capable of operation with high overall efficiency. The principal difficulty with the dc approach is that it is limited to low electron-beam energy and, therefore, long laser wavelengths (>10 $\mu \mathrm{m})$.

In our view, the best approach is to use an $r f$ linac to achieve high electron-beam energy and correspondingly short wavelengths. The spent 


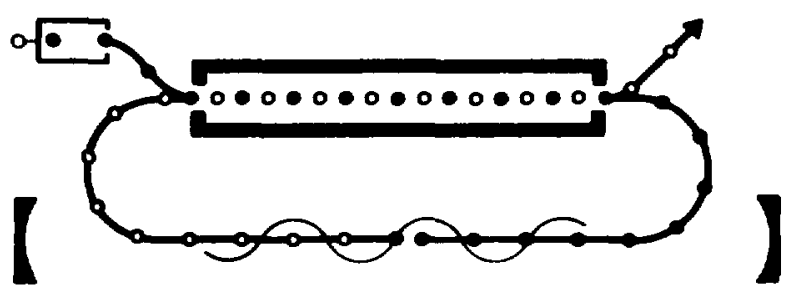

EFFICIENCY:

$\begin{array}{ll}\text { ENERGY } \sim 100 \mathrm{MOV} & \text { LASER/O-GEAM } \sim 10 \% \\ \text { POWER } \sim 100 \mathrm{~kW} & \text { LASER/rf } \sim 40 \% \\ \text { WAVELENGTH } \sim 1 \mu \mathrm{m} & \text { LASER/dC } \sim 25 \%\end{array}$

Fig. 13.

This figure summarizes the operation of a high-efficiency free electron laser with a racetrack $r f$ accelerator/decelerator energy recovery system. The laser extraction efficiency (laser energy out divided by electron-beam energy in) is predicted to be $10 \%$ for values of the laser field which can be achieved in a practical, high-average-power device with present optics and accelerator technology. By recovering the leftover energy, the overall (dc power to laser power) efficiency is increased to $25 \%$.

time the bunches are inserted $180^{\circ}$ out of phase from the microwave field--in between the bunches being accelerated. In this way, the electrons are decelerated, and return their remaining energy directly to the accelerating fields. The energy recovery process can be rather efficient. The only loss in the deceleration process is the energy remaining with the electrons when they are dumped. The result is an energy recovery system capable of operating with high electron energy ( $>100 \mathrm{MeV}$ ), and correspondingly short wavelength ( $1 \mathrm{\mu m}$ ), at high average power. For a system in which $10 \%$ of the electron-beam energy is extracted as laser energy in the wiggler, the overall efficiency can be $25 \%$ or more. An artist's conception of a 100-kW FEL laser based on this approach is shown in Fig. 14 .

The performance figures summarized in Fig. 13 are the result of extensive numerical calculations. The laser extraction efficiency was calculated using the FEL codes described in Sec. II below. The laser parameters are summarized in Table III. The accelerator performance was calculated using the code SUPERFISH. 


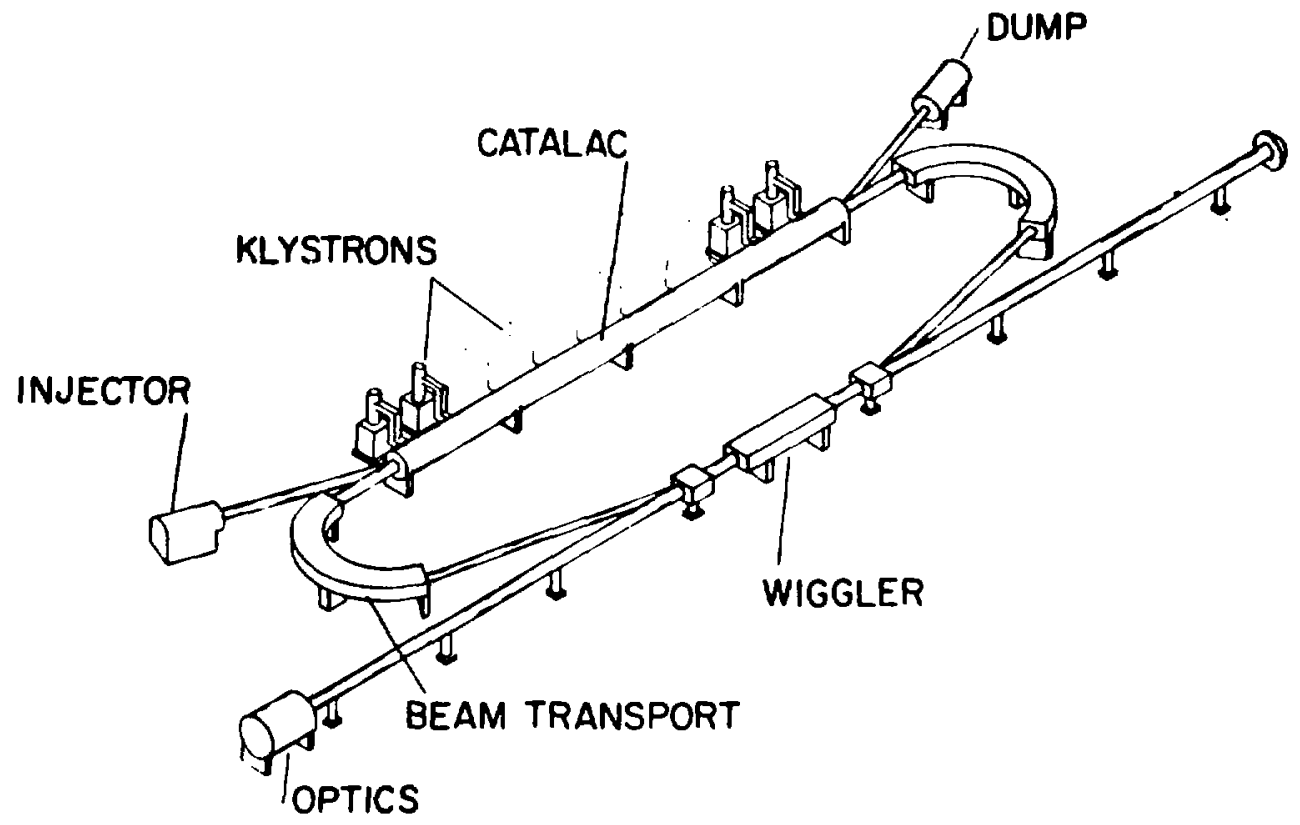

Fig. 14 .

This figure shows an artist's conception of a free electron laser with racetrack $r f$ accelerator/decelerator energy recovery system.

TABLE II I

100-kW AVERAGE POWER, 1- $\mu \mathrm{m}$ FREE-ELECTRON LASER

(a) ACCELERATOR PARAMETERS

rf pulse length

Electron-beam pulse length

Repetition rate

Accelerator frequency

Acceleration in injector

Acceleration (deceleration) in CATALAC

Total acceleration

Total accelerator length (CATALAC and injector)

Total accelerator dissipation (copper losses)

Average electron energy at dump

Losses in beam dump

Average current

Peak current
$130 \mu \mathrm{S}$

$100 \mu \mathrm{S}$

$100 \mathrm{~Hz}$

$440 \mathrm{MHz}$

$17 \mathrm{MeV}$

$83 \mathrm{MeV}$

$100 \mathrm{MeV}$

$100 \mathrm{~m}$

$2 \mathrm{MW}$

$8 \mathrm{MeV}$

$8 \mathrm{MN}$

$1 \mathrm{~A}$

$250 \mathrm{~A}$ 
TABLE III (cont)

(b) WIGGLER PARAMETERS

$\begin{array}{lc}\text { Microbunch length }\left(11^{\circ}\right) & 70 \mathrm{ps} \\ \text { Microbunch repetition rate (8th subharmonic } & 55 \mathrm{MHz} \\ \text { Electron beam diameter } & 1.4 \mathrm{~mm} \\ \text { Energy spread } & 0.5 \% \\ \text { Emittance } & 0.4 \pi \mathrm{mm}-\mathrm{mrad} \\ \text { Wiggler length } & 25 \mathrm{~m} \\ \text { Wiggler period } & 4-3 \mathrm{~cm} \\ \text { Magnetic induction (rms) } & 3-4 \mathrm{kG} \\ \text { Initial mean electron energy } & 100 \mathrm{MeV} \\ \text { Final mean electron energy } & 90 \mathrm{MeV} \\ \text { Extraction efficiency } & 10 \% \\ \text { Final trapped electron energy } & 85 \mathrm{MeV} \\ \text { Trapping fraction } & 60 \%\end{array}$

(c) LASER PARAMETERS

Wavelength

Micropulse length

Micropulse repetition time

Average power

Average power during $100_{-\mu \mathrm{s}}$ laser pulse

Peak power in micropulse

Saturated ga in per pass

rf-to-laser efficiency

Klystron efficiency

dc-to-laser efficiency
$1 \mu \mathrm{m}$

85 ps

$14 \mathrm{~ns}$

$100 \mathrm{~kW}$

$10 \mathrm{MW}$

$2.5 \mathrm{GW}$

$40 \%$

$40 \%$

$65 \%$

$25 \%$

The electron distribution function emerging from the wiggler is shown in Fig. 15. Note that the emergent distribution function is dominated by two peaks and has no very slow electrons. This enormously simp?ifies the design of the beam transport system and the decelerator. The two peaks represent those electrons that have passed through the wiggler relatively unaffected and those that have been "trapped" and strongly decelerated in the moving force field created by the laser and wiggler. In transporting the electrons back 


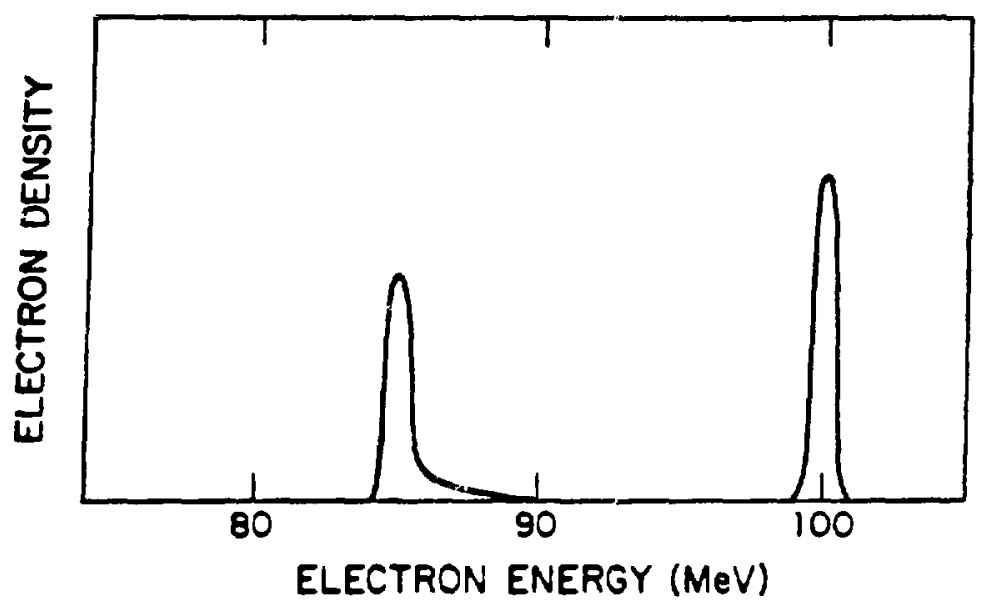

Fig. 15.

The electrons emerging from the free-electron laser fall into two groups. One group, near the original energy (100 MeV), corresponds to electrons which, because of their phase upon entering the wiggler field, was not trapped and experienced only a small velocity change. The other group, near $85 \mathrm{MeV}$, corresponds to electrons which were trapped in the field of the laser and wiggler and decelerated to a substantially lower energy.

from the laser to the linac for deceieration, the electrons with different energies must arrive at the linac at the proper phase of the rf fields. This may be accomplished with the beam transport system shown in Fig. 16. To extract the maximum energy from both groups of electrons, the accelerator structure is split into two sections called the catalytic linac (CATALAC) and the post linac (POLAC), as in Figs. 16 and 17. The low-energy electrons are decelerated to about $2 \mathrm{MeV}$ in the CATALAC section and then dumped, and their energy used to accelerate new electrons. The high-energy electrons are likewise decelerated in the CATALAC. To recover their remaining energy, they may be further decelerated to about $2 \mathrm{MeV}$ in the POLAC before they are dumped. This may, however, lead to problems of stability and control and was not done in calculating the performance for Fig. 13 and Table III. Because the current being decelerated in the POLAC is less than the current being accelerated (the total current), rf power must be added to the POLAC to offset the net beam loading. The rest of the system performance is summarized in Tatle III. The dc-to-rf conversion efficiency is assumed to be $65 \%$, equal to that of a modern Valvo high-power klystron. More efficient tubes (gyrocons) are now being developed at LASL. 


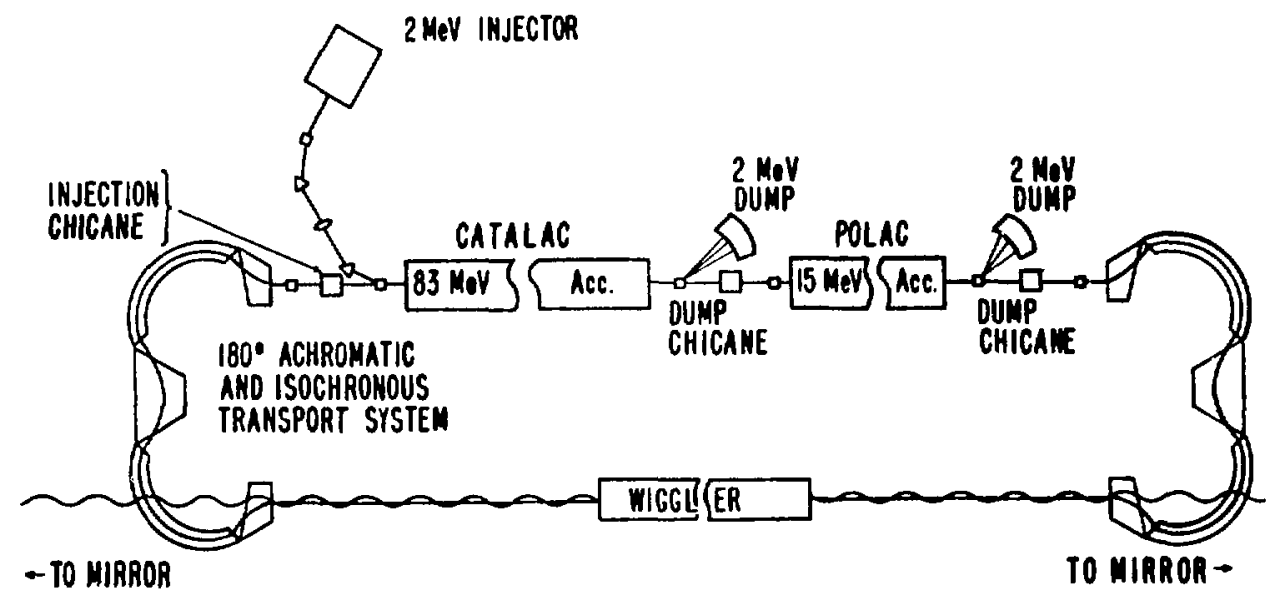

Fig. 16 .

Schematic diagram of an achromatic, isochronous transport system for a high efficiency free electron laser. In this configuration, the accelerator/ decelerator system is split into CATALAC (catalytic-linac) and a POLAC (post-linac) sections. By providing two beam dumps, the electrons can all be decelerated to a low energy before being dumped to maximized the efficiency.

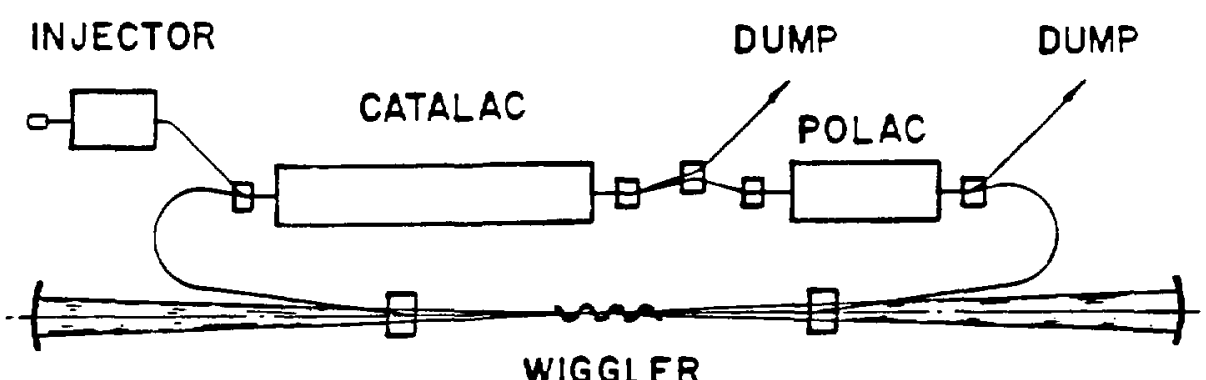

Fig. 17.

Alternative configuration of a racetrack free electron laser system with the accelerator/decelerator split into CATALAC and POLAC sections. The electrons are decelerated by the CATALAC and then all are dumped. This decreases the efficiency relative to Fig. 16, but improves the stability.

\section{B. Technology Development Areas}

Although the overall performance prejictions are believed to be achievable, areas remain where development is required. These areas include wiggler design, accelerator technology, and optical design. The projected performance of the high-efficiency wiggler depends on trapping and decelerating the electron in a high-intensity laser field with a properly tapered wiggler field. 
Although the theoretical calculations described below are believed to be fundamentally correct, this type of wiggler performance has not yet been tested experimentally. Moreover, the effects of transverse momentum spread in the electron beam and transverse nonuniformities in the wiggler magnetic field must be treated more accurately than is done in Sec. II below. Thus, further theoretical and experimental work must be done to confirm the FEL performance predictions.

Similarly, while the calculated losses and overall accelerator performance figures are believed to be quite accurate, several difficulties will have to be faced in developing the accelerator system; namely, design of the injector and accelerator structure to achieve high current with small momentum anu energy spread, and cooling of the $\mathrm{cw}$ accelerator resonant cavities. The required average current has been achieved in pulsed accelerators with nearly the required energy spread and transverse momentum spread (emittance), and the required peak current has been obtained with nearly the required energy spread, but with somewhat greater emittance. At the present time, the origins of emittance in accelerator injectors are not well understood, and work must be done to develop high-peak-current, low-emittance injectors. This is important because, as shown below, the shortest achievable wavelength is proportional to the emittance. In addition, this same performance remains to be demonstrated in $\mathrm{cw}$ accelerators where lower gradient structures will be needed for cooling.

Figure 18 shows the optical system, which consists of a simple negative branch unstable resonator. The laser beam is focused in the center of the

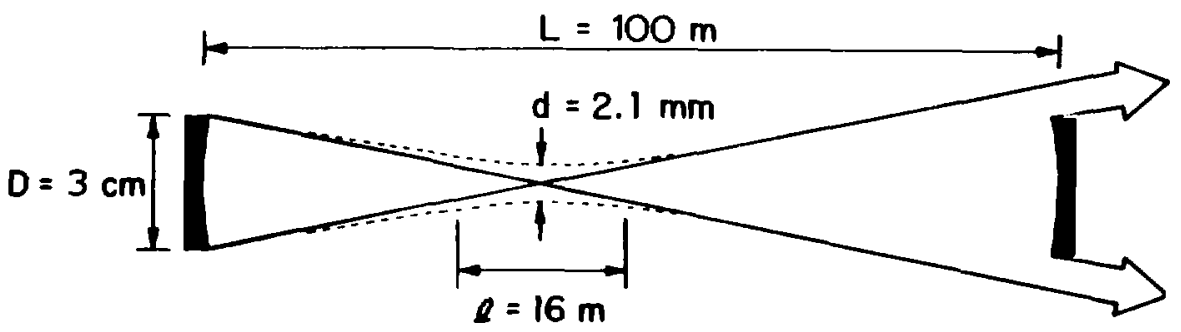

Fig. 18.

A simple negative-branch unstable resonator $c$ an be used to focus the laser beam to a high inside the wiggler. 
cavity to pass through the wiggler with a high enough intensity to trap the electrons, as discussed below, and extract energy efficiently from the electron beam. The laser beam is further intensified by compressing it into short bunches that coincide with the electron bunches emerging naturally from the rf linac. Because the equivalent Fresnel number of this cavity is near unity, corresponding to a near-concentric resonator, it may be advantageous to use a diffraction-coupled, stable resonator to reduce the output coupling without reducing the far-field brightness. Lower-output coupling would increase the intensity in the wiggler and improve the energy extraction. Increased energy extraction, in turn, would simplify the accelerator design by reducing the peak and average current, as discussed above. Up to now, resonator design has not been studied in detail. Problems of alignment sensitivity and tolerance toward thermal distortion have been treated only very simply. However, optical problelis do not seem to present a fundamental stumbling block to the development of a high-power FEL.

Because the electron beam passes through the linac twice, once while being accelerated and once while being decelerated, the average current in the linac during the macropulses is $2 \mathrm{~A}$. A linear accelerator $100 \mathrm{~m}$ long with a 2-A beam current circulating through it will be very susceptible to beam breakup caused by the $\mathrm{HEM}_{11}$ beam breakup modes as well as other higher order modes. Special care will be needed in the accelerator structure design to spoil the $Q$ of the $H_{11}$ modes and to use methods that vary the HEM 11 resonant characteristics to avoid coherent interaction along the beam center line. The disk-and-washer structure seems to be particularly adaptable to these methods. These methods, plus strong focusing between accelerator sections, should make it possible to raise the beam breakup threshold well above 2 n. Another useful technique that can be used is rotating the beam by $90^{\circ}$ after the first pass through the linac. This guarantees that the subsequent pass cannot regeneratively interact with fields produced by the first-pass beam. This problem requires more study to make sure that beam breakup will be controlled.

When the Polac is used to recover energy from the high-energy electrons, a potential instability of the rf recovery system, not present in a single-pass laser system, arises from fluctuations in the laser power, such as might be caused by thermal distortion of the laser optics. If, for example, the laser power in the cavity decreases, the wiggler capture fraction will decrease, 
allowing more electrons to pass through the POLAC. This, in tur:1, will put more $r f$ power into the POLAC and cause the electrons to be accelerated to a higher energy. Because this will bring them into the wiggler off resonance, the gain will decrease, leading to a further decrease in laser power. For small fluctuations in the electron energy, this is a second-order effect, because the electron energy is initially at the peak (resonant) value.

Thus, the systom will be stable because the saturation behavior of a tapered wiggler (like ary normal laser) causes the gain to increase linearly with decreasing laser power, at least for small variations. However, for large excursions of the electron energy (greater than a couple of tenths of a per cent in the 100-kW design) the situation becomes unstable. Thus, the POLAC must be controlled to prevent such large fluctuations. The response time of the rf fields in the POLAC is expected to be approximately a few microseconds. This should be faster than the laser fluctuations, because the cavity roundtrip time is $-2 \mu \mathrm{s}$. The stability limits can be enhanced by forcing the laser to oscillate at a wavelength slightly shorter than the peak. Alternatively, the laser can be made absolutely stable against this instability by dumping all the decelerated electrons after they emerge from the CATALAC, before they pass through the POLAC, as shown in Fig. 17. For the present design this approach was selected. Even so, the overal efficiency is found to be $25 \%$. Clearly, much work must done on the stability and control of such a laser system to ach ieve the maximum power and efficiency.

In summary, the 100-kW conceptual design study points cut a number of areas where development is required and better trade-offs should be made. However, the problems appear to be solvable with relatively modest developments of present technology. No inventions are necessary. In fact, the results indicate that average powers even higher than $100 \mathrm{~kW}$ are possible with reasonable extrapolations from the present state of the art. Thus, the idea of a high-power FEL operating at a useful wavelength seems quite feasible.

c. Objectives of the Present Program

As described above, developments are required in our understanding of FEL physics, in the technology of high-average-power $\mathrm{CW}$ accelerators, and in the design of appropriate optical resonators. However, of these three problem areas the first, FEL physics, is the most fundamental and deserves first priority. In particular, the predicted perfornance of adiabatically decelerating wigglers has never been tested experimentally. Because all approaches 
to hign power and efficiency at short wavelengths depend on nonuniform wiggiers, the principal objective of the present program has been to design a suitable experiment to test the theory and to demonstrate high extraction efficiency (a few per cent or more). A second objective of the program has been to develop the theory in greater detail to examine some of the assumptions used and to include some of the nonideal effects such as transverse electron momenta and magnetic field nonuniformities. Because the theoretical capabilities developed in this program were used in the design of the experiment, it is most convenient to describe the theoretical developments first. Below the theoretical results are summarized in Sec. II, the experimental design is described in Sec. III, and the conclusions and recommendations are summarized in Sec. IV.

\section{THEORY}

Three theoretical efforts were pursued under the present contract. The first effort was to develop an accurate, self-consistent single-particle theory of the free electron capable of describing coherent and nonlinear effects. The second effort was to develop a multiparticle theory based on two-dimensional particle-beam codes, with which to examine plasma effects as well as multidimensional effects. The third effort was to use simpler methods--analytical theories backed up by computer calculations--to study the optimization and scaling of FELS.

A. Single-Particle Theory

Theoretical work on the FEL from the single-particle point of view has been carried out in collaboration with Professor William H. Louisell of the University of Southern California. This work has concentrated on obtaining a formalism for the nonperturbative, self-consistent solution of the Maxwell equations (for the laser electric field) coupled with the Boltzmann equation (for the electrons), with the objective of understanding pulse-propagation, saturation, and focusing effects that may influence the energy extraction and efficiency of a FEL with a variable wiggler. The theories of the FEL published by the Arizona group 51,52 are perturbative, in the sense that the Boltzmann distribution function is expanded to lowest order in the laser field about an initially uniform electron distribution. This is not appropriate for tightly trapped electrons as anticipated in FELs with tapered wigglers. Indeed, the pulse-propagation phenomena which occur for tightly trapped electrons may 
differ importantly from the propagation phenomena investigated by the Arizona group for untrapped electrons, ${ }^{53}$ because it may be expected that Raman sidebands will develop in the laser electric field cwing to the nearly periodic motion of the trapped electrons. To understand this effect quantitatively, it will be necessary to obtain a self-consistent solution for the laser electric field and electron distribution. Other published approaches to laser pulse propagation in FELs based on incrementing the laser field energy by the amount of energy lost or gained by the electrons are not capable of including changes in the phase or frequency of the laser field, and hence are not satisfactory for calculations on the type of FEL to be built at Los Alamos.

During the last year, a numerical metrod based on following the motion of representative particles has been developed for the self-consistent, nonperturbative solution of Maxwell's equations and the electron equations of motion, including longitudinal variation of the wiggler magnetic field and both transverse and longitudinal variation of the laser electric field, but excluding the effects of space charge. This formalism includes the generation of new frequencies and pulse-shaping phenomena, and transverse variation of both the laser electric field and the particle orbits. We have rleveloped a numerical implementation of this theory, using a technique of summation over particle trajectories, and have partially tested the numerical methods using a special class of wiggler designs.

The new results agree with the earlier theories where the latter are applicable. However, the new computational capabilities make it possible to treat the propagation of short pulses in an unsteady-gain medium consisting of electron microbunches from an $r f$ linac, nonlinear focusing effects, and coherent excitation of electron synchrotron oscillations in the laser/wiggler field, as well as radial laser-field variations. We expect that the inclusion of the radial dependence of the laser electric field and the electrons' transverse motion will have a major effect on the particle dynamics and field evolution of the FEL, particularly on particle trapping; the escape of trapped particles; and the focusing, defocusing and phase shift of the field as the result of propagation; mode development in oscillators.

B. Multiparticle Theory

When the electron density is high, electron-electron interactions (collective, or plasma oscillations) become important. ${ }^{43-46}$ A self-consistent two-dimensional plasma simulation code CCUBE has been implemented to simulate 
FELs in realistic geometries, including plasma effects. Because this largescale particle-simulation code solves the particle dynamics and electromagnetic field quantities self-consistently, it can actually be applied to study the nonlinear interaction of FELs in all the regimes of interest. The principal limitation of this approach is that core storage and computer-time requirements increase with the ratio of the wiggler and optical wavelengths; that is, as $\gamma^{2}$. Thus, the approach is most useful for low $\gamma^{\prime} s$, below $\sim 10$.

Several simulations have been done to study two-dimensional effects in FELs. The results show the existence of waves propagating obliquely with respect to the direction of electron beam propagation. These off-axis modes could have significant effects on the lasing mechanism when the FEL is operated in an oscillator mode. However, our study shows that the two-dimensional effects are not important in an amplifier mode because of the dominance of the input signal. The importance of two-dimensional effects is found to be greatest at low values of $\gamma$. Also we have investigated in detail the dependence of the efficiency of FELs with uniform magnetic wigglers on the intensity of the electromagnetic radiation. If one operates the FEL as an amplifier, there exists an optimum intensity of the input electromagnetic radiation. In the oscillator mode, the efficiency of energy extraction from the electron beam decreases rapidly if the intensity of radiation inside the cavity exceeds the optimum level. We have developed a theory to predict the optimum radiation intensity and it agrees with the simulations very well.

A suggested extension of these studies over the next twelve months is:

(1) Effects of finite radial profiles of electron beam and laser beam.

(2) Two-dimensional effects in oscillators, in particular during start-up. The numerical simulation approach is uniquely suited to consideration of start-up from noise, and is therefore of great interst for FELs of the type being developed at Naval Research Laboratory (NRL), which will operate in short pulses. We hope that useful calculations can be done for the conditions of the NRL experiments where comparison with experimental data will be possible.

(3) Development of novel numerical techniques to study the interaction of ultrarelativistic electron beams with electromagnetic radiation, so that higher values of $r$ may be usefully considered.

(4) Performance of variable wigglers; in particular, irapped particle dynamics, space-charge effects, optimization, and scaling laws. 
(5) Coherent pulse propagation. This is important in rf linacs where the electron beam and, therefore, the laser beam, is in the form of a train of very short ( 25 ps) pulses.

C. Optimization of Laser Performance

To optimize the performance of a FEL, it is necessary first to define the meaning of optimum. Because the many systelıs trade-offs involving optics, energy recovery, and other characteristics are still too complex and poorly understood to include them in a real optimization, the definition of optimum used for this study is simply the maximum extraction efficiency for a given incident laser wavelength and power. The geometry of the wiggler region, which must be varied to achieve this optimum, is shown in Fig. 19.

To carry out the optimization in an economical fashion, the adiabatic theory first used by Morton ${ }^{49}$ is used to calculate the performance. This is justified on two grounds. First, the performance near an optimum is only a weak function of the parameters, so that errors introduced by the approximation will not significantly affect the performance. Second, numerical calculations show that for the conditions of interest in this work, the adiabatic approximation produces results accurate to better than $10 \%$.

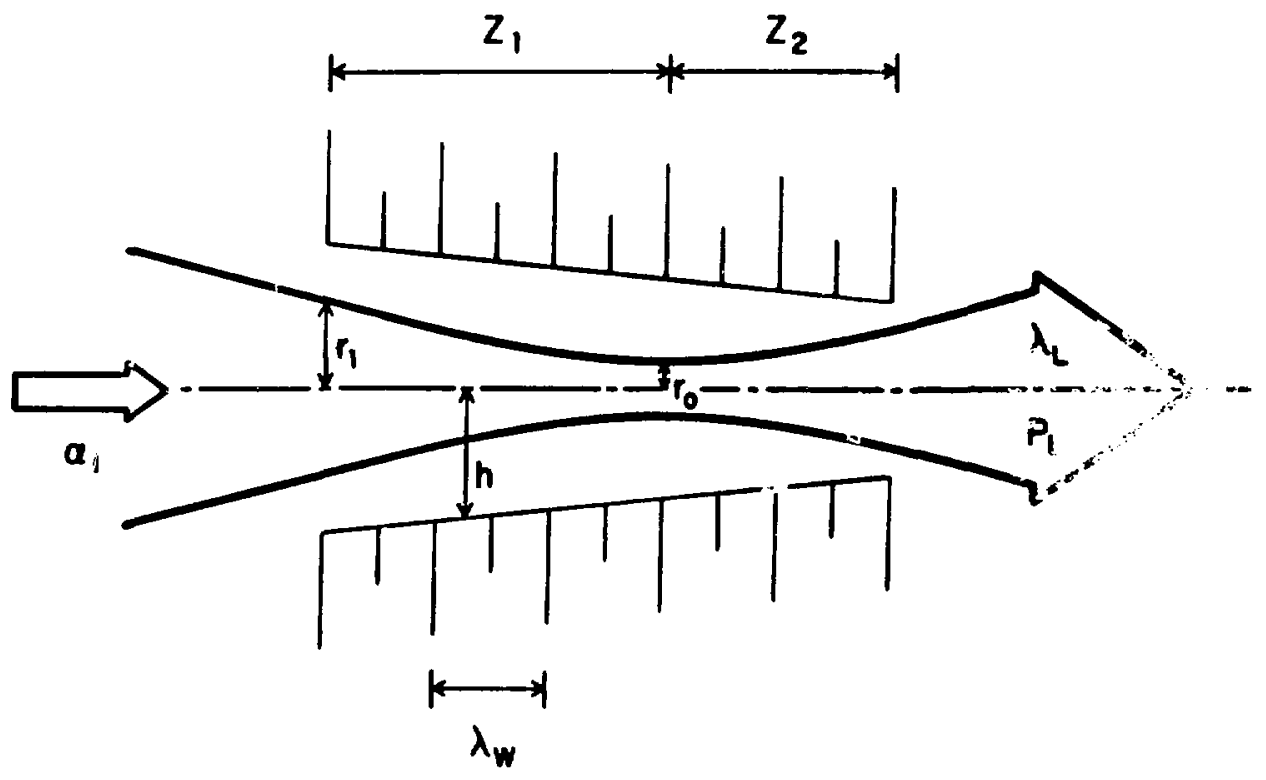

Fig. 19.

Geonetry of the wigg ler region. 
In the adiabatic approximation ${ }^{49}$ the extraction efficiency $n_{x}$ may be expressed as the product

$$
n_{x}=n_{C} n_{0}
$$

Here, $n_{c}$ is the capture efficiency; that is, the fraction of the electrons in the beam that are trapped in the laser/wiggler field and decelerated, and $n_{D}$ is the deceleration efficiency; that is, the fraction of the original energy removed from the trapped electrons. It is easy to show that the performance then depends only on the parameter

$$
p=P_{L} / P_{0}
$$

where $P_{L}$ is the laser power and

$$
P_{0}=\left[\frac{n\left(1+a_{\left.W^{2}\right)}\right.}{4 a_{W}} \frac{m c^{2}}{e}\right]^{2} \frac{4 \pi}{Z_{0}}
$$

is called the characteristic power. Here, $m$ is the electron mass, $c$ the velocity of light, $e$ the electron charge, $z_{0}$ the impedance of free space (mks units are used), and $n$ is the ratio of the wiggler-entrance half-height $h$, to the wiggler-entrance laser-bcam radius $r_{1}$. Generally, this should be about 2 (or more) to avoid diffraction effects. The dimensionless magnetic vector potential of the wiggler is defined by the expression

$$
a_{W}=\frac{e W^{\lambda} W}{2 \pi m c}
$$

in which $B_{W}$ is the rms magnetic field strength and $\lambda_{W}$ is the wiggler period. The optimal performance is shown in Fig. 20, where we see that it is a monotonicaily increasing function of $P_{L} / P_{0}$, varying very nearly as the square root of this quantity. The optimum is obtained for $a_{W}=1$, in which case (using $n=2$ ), the characteristic power is $P_{0}=8.71 \mathrm{GW}$. Clearly, to achieve significant efficiency from a FEL, it will be necessary to use peak laser fluxes of the order of gigawatts. This, of course, places a burden on the mirrors forming the optical cavity. One of the important trade-offs in the design of high-power FELs will be in optical design for high intracavity flux. 


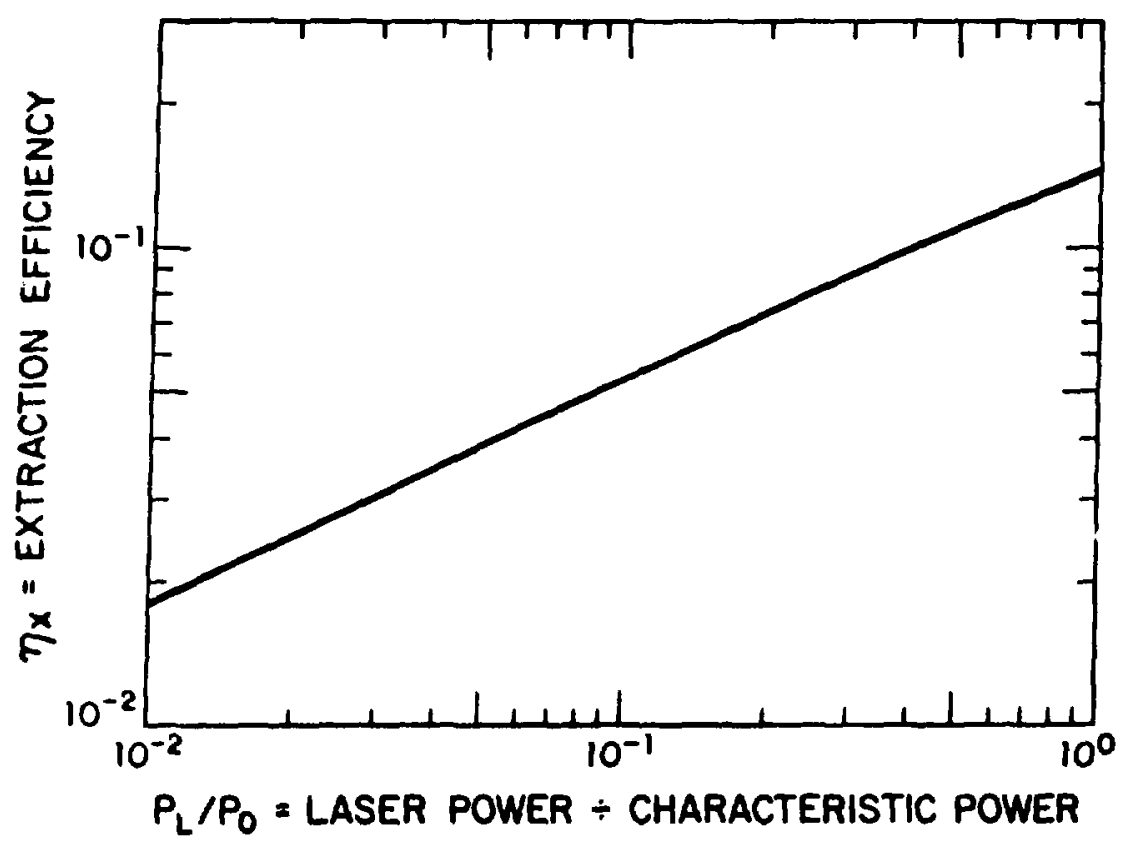

Fig. 20.

Optimized laser performance (extraction efficiency) as a function of $P_{L} / P_{O}$.

The optimum wiggler geometry is also a function of $p$ alone, and can be described by the factors

$$
\begin{aligned}
& g=r_{1} / r_{0}, \\
& y=\lambda_{W 2} / \lambda_{W 1},
\end{aligned}
$$

where $r_{0}$ is the laser beam radius at the focus and $\lambda_{W 1}$ and $\lambda_{W 2}$ are the wiggler periods at the entrance and exit, respectively. The factor $g$ plays the role of the Fresnel number of the wiggler region, and the factor $y$ describes the deceleration of the trapped particles, because the energy of the trapped particles is proportional to the square root of the wiggler wavelength. The factors $g$ and $y$ are plotted in Fig. 21. These figures contain essentially all the data required for the optimal design and performance of FELs.

Applying these results to the 100-kW, 1-um FEL described in Sec. I above leads to a wiggler length of $21 \mathrm{~m}$. To shorten the wiggler length, an offoptimum design corresponding to $g=3.22$, rather than the optimum $g=2.17$, 


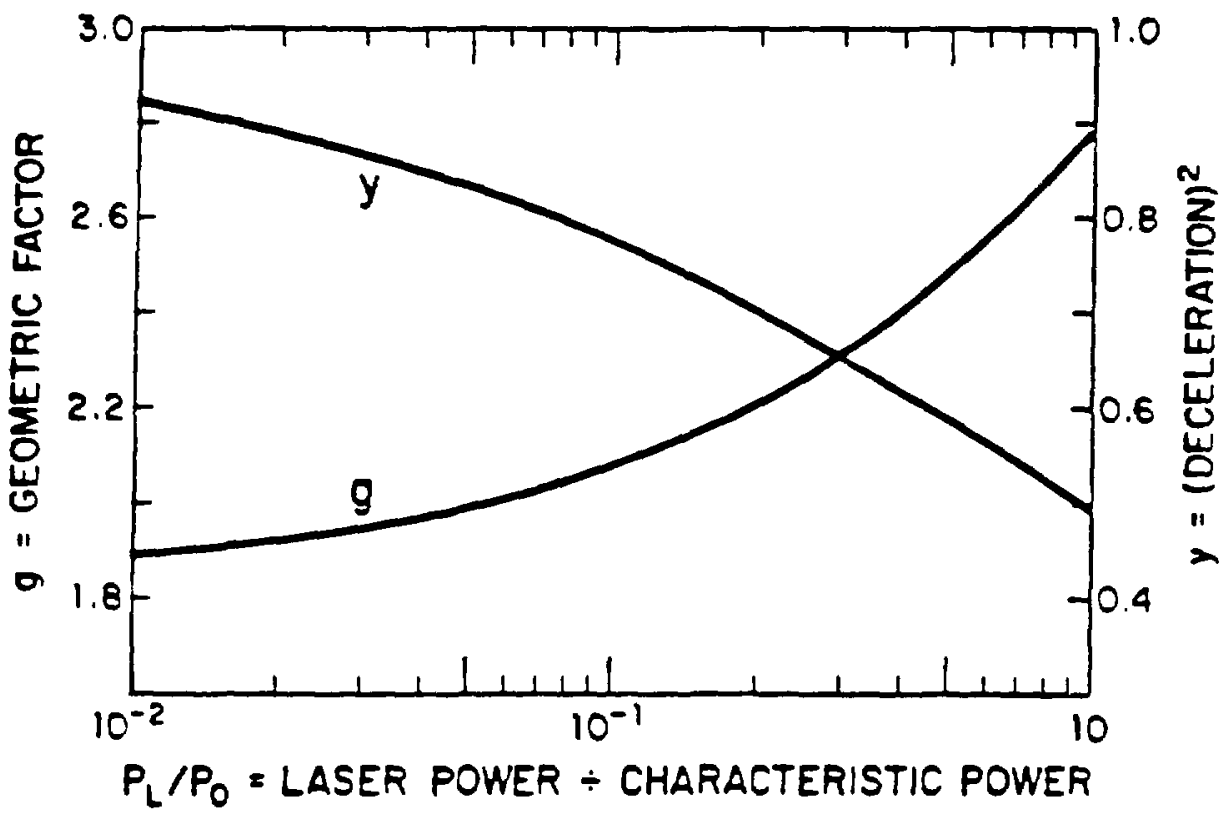

Fig. 21.

Optimized wiggler parameters as a function of $P_{L} / P_{0}$.

was chosen. This reduced the wiggler length to $16 \mathrm{~m}$, but did not significantly reduce the extraction efficiency. In applying these results to the design of the 10- $\mu \mathrm{m}$ experiment, a reduced magnetic vector potential $a_{W}=0.5$ was chosen, rather than the optimum value $a_{W}=1$. This was done because available permanent-magnet materials cannot produce the magnetic fields required to give $a_{W}=1$ under the conditions of the proposed experiment, which correspond to $r$ ather short magnet periods.

D. Effects of Transverse Electron Momentum

At the present time, accurate calculations can be made for an electron beam having a finite energy spread. Similar calculations for an electron beam having a finite transverse spread are not yet possible. However, the importance of transverse effects can at least be estimated by comparing them with an "equivalent" energy spread in the following manner. The bandwidth (Doppler width) caused by nonresonant electrons introduced by an energy spread $\Delta y$ in the electron beam is given by the expression

$$
\Delta \lambda_{L} / \lambda_{L}=2 \Delta \gamma / \gamma,
$$


as shown in the theory of small signal gain in tapered wigglers. 54 Similarly, the bandwidth introduced by a transverse momentum spread is given by the expression

$$
\Delta \lambda_{L} / \lambda_{L}=\gamma^{2} \Delta B_{\perp}^{2} /\left(1+a_{w}^{2}\right)
$$

where $C \Delta B_{\perp}$ is the width of the transverse velocity distribution function as defined in the small signal gain theory. 54 Comparing Eqs. (9) and (10), we see that the nonresonance effect introduced by transverse velocities is equivalent to that of an energy spread given by the formula

$$
\Delta Y /\left.Y\right|_{B_{1}}=(1 / 2) \gamma_{\Delta \beta_{1}}^{2} /\left(1+a_{W}{ }^{2}\right) .
$$

This same approach may be used to estimate the importance of nonresonances introduced by electrons off the wiggler axis where the wiggler field is stronger. Near the axis of a linearly polarized wiggler, the wiggler field varies according to the expression 55

$$
a_{W} \cong a_{W 0}\left(1+(1 / 2) k_{W}^{2} r^{2}\right)
$$

But the resonant laser wavelength satisfies the condition

$$
k_{L}=2 \gamma^{2} k_{W} /\left(1+a_{W}^{2}\right)
$$

Proceeding as before, we find that the "equivalent" energy spread is therefore given by the expression

$$
\left.\frac{\Delta Y}{r}\right|_{r_{B}}=\frac{1}{2} \frac{a_{W^{2}}}{I+a_{W^{2}}} k_{W}^{2} r_{B}^{2}
$$

where $r_{B}$ is the radius of the electron beam.

Because the transverse momentum and beam radius are related, it is not possible to minimize them independently. In fact, the emittance,

$$
\varepsilon=\pi \Delta B \quad r_{B},
$$


is generally preserved in any good electron focusing system. The total equivalent energy spread caused by transverse effects, which is what we wish to minimize, is probably satisfactorily approximated by the formula

$$
\left(\Delta \gamma /\left.\gamma\right|_{\perp}\right)^{2}=\left(\Delta \gamma /\left.\gamma\right|_{B_{\perp}}\right)^{2}+\left(\Delta \gamma / \gamma \mid r_{B}\right)^{2}
$$

The minimum value of this expression has the value

$$
\left.\frac{\Delta \gamma}{\gamma}\right|_{\perp}=\sqrt{2} \frac{\gamma \in a_{W}}{\lambda_{W}\left(1+a_{W}{ }^{2}\right)}=\frac{1}{\sqrt{2}} \frac{a_{W}}{\gamma \lambda_{L}} \text {. }
$$

A measure of the importance of transverse effects is provided by comparing the total equivalent energy spread with the height of a decelerating bucket. Using the adiabatic approximation for the bucket height, $\Delta y / \gamma / \hat{p}$, we find that the importance of transverse effects is represented by the parameter

$T_{R}=\frac{\Delta r /\left.r\right|_{1}}{\Delta r /\left.\gamma\right|_{\hat{p}}}=\frac{\varepsilon}{\Delta n \lambda_{L}}\left(\frac{P_{0}}{P_{L}}\right) 1 / 4\left\{\frac{1+a_{W^{2}}}{2 a_{W^{2}}}\left[\cos \psi_{r}-\left(\frac{\pi}{2}-\psi_{r}\right) \sin \psi_{r}\right]\right\}^{-1 / 2,}$

where $\Psi_{r}$ is the so-called resonant phase of the decelerating electrons. $T_{R}$ must be less than unity for efficient trapping. We see from Eq. (18) that the shortest wavelength that can be achieved in a high-efficiency laser is determined by the emittance. The energy $r$ does not enter directly, but only through the fact that $\varepsilon$ ordinarily improves like $1 / \gamma$. Unfortunately, the optimum wiggler length varies as $\gamma^{4} \lambda_{L}$, whereas the cavity length varies as $r^{2}$. This makes higher $r$ a hard way to get shorter wavelengths. Likewise, we see from Eq. (18) that the achievable wavelength decreases only as the one-fourth power of the laser power, making this a difficult way to get shorter wavelengths. Where does this leave us in terms of practical, achievable emittances? As a rule of thumb, the emittance of good linacs can be estimated from the formula

$$
\varepsilon=60 \times 10^{-6} \pi \quad \bar{I}^{1 / 2} / \gamma,
$$


where $I$ is the average current (amperes) and $\varepsilon$ is in meter-radians. Using Eqs. (18) and (19), together with the baseline conditions of the high-power laser (average current $=1 \mathrm{~A}$ ), we find that the wavelength limit imposed by the emittance is

$$
\lambda_{\mathrm{L}}>0.3 \mu \mathrm{m}
$$

Thus, wavelengths around $1 \mu \mathrm{m}$ appear to be within the state of the art. The origin and cure of emittance is not well understood at the present time. However, the emittance is believed to be determined by the gun, and most likely by the grid structure. Improvements in emittance are to be experted, particularly if the grid can be improved or eliminated (for $\mathrm{cw}$ machines), and correspondingly shorter wavelengths should then be possible.

\section{EXPERIMENTAL DESIGN}

The object of the FEL gain experiment at $10.6 \mu \mathrm{m}$ is to demonstrate the operation of a high-efficiency tapered wiggler. Gain versus input laser power and the number of electrons trapped and decelerated by the wiggler and laser fields will be measured from weak-signal to saturation conditions.

The experimental parameters are summarized in Table IV. The design of the experiment reflects two considerations. In the first place, the available $r f$ power is not sufficient to accelerate the present peak current of the linac (>20 A) to $20 \mathrm{MeV}$. This could be circumvented by reducing the average current and bunching at a subharmonic of the accelerator frequency. Alternatively, short $(<10-n s)$ pulses can be accelerated using the microwave energy stored in

\section{TABLE IV}

\section{BASELINE PARAMETERS FOR 10 $1 \mathrm{~m}$ LASER}

Electron energy

Micropulse peak current

Micropulse length

Energy spread (full width)

Emittance

Equivalent energy spread

Effective total energy spread

$$
\begin{aligned}
& \gamma=40 \\
& I=25 \mathrm{~A} \\
& \tau_{p}=25 \mathrm{ps} \\
& \Delta \gamma / \gamma=2.0 \% \\
& \varepsilon=2 \pi \mathrm{mm}-\mathrm{mr} \mathrm{ad} \\
& \Delta \gamma / \gamma / \perp=0.56 \% \\
& \Delta \gamma / \gamma / \mathrm{T}=2.1 \%
\end{aligned}
$$




\section{TABLE IV (cont)}

Electron beam radius

Laser wavelength

Laser micropulse power

Radius at focus

Rayleigh range

Wiggler length before focus

Wiggler length after focus

Wiggler vector potential (rms)

Resonant phase

Resonant energy

Wiggler wavelength

Wiggler magnetic induction ( $r m s$ )

wiggler half-aperture
$r_{B}=0.81 \mathrm{~mm}$

$\lambda_{\mathrm{L}}=10 \mu \mathrm{m}$

$P_{L}=1.5 \mathrm{GW}$

$r_{0}=0.97 \mathrm{~mm}$

$z_{0}=0.30 \mathrm{~m}$

$\mathrm{z}_{1}=0.55 \mathrm{~m}$

$z_{2}=0.37 \mathrm{~m}$

$a_{W}=0.5$

$\Psi_{r}=37.8^{0}$

$r_{r}=40 \rightarrow 35$

$\lambda_{W}=26 \mathrm{~mm} \rightarrow 20 \mathrm{~mm}$

$B_{W}=0.21 \mathrm{~T} \rightarrow 0.27 \mathrm{~T}$

$h=4.1 \mathrm{~mm} \rightarrow 3.1 \mathrm{~mm}$

the accelerator cavities. This latter approach was chosen because it is quicker and less expensive to implement and because the short pulses will produce less radiation. This is an important consideration in the present shielding enclosure, which was designed for 5 -MeV operation.

The second major consideration is the low gain expected from highextraction-efficiency tapered wigglers. This gain, of the order of 1-2\% peak, will be even lower when averaged over the several-ns pulse, because of the small duty factor of the electron bunches (about $15^{\circ}$ out of each $\mathrm{rf}$ cycle). Because the individual microbunches (about 30 ps long) cannot be completely resolved with an oscilloscope, the observed gain will be smaller than the peak gain. Thus, very sensitive measuring techniques will be required. The details of the experiment are described in the following paragraphs.

The gain experiment is shown schematically in Fig. 22. A single $\mathrm{CO}_{2}$ laser pulse is focused through the wiggler magnet (comprised of $\mathrm{SmCO}_{5}$ permanent magnet units) concurrent with the electron-beam pulse. To enable sensitive detection of only the stimulated energy gained from the electrons, the input laser pulse is plane polarized at $45^{\circ}$ to the linear wiggler field and a crossed polarizer (stacked germanium plates with extinction ratio of $10^{4}$ to $10^{5}$ ) is placed in the collimated exit beam to remove the $\mathrm{CO}_{2}$ 


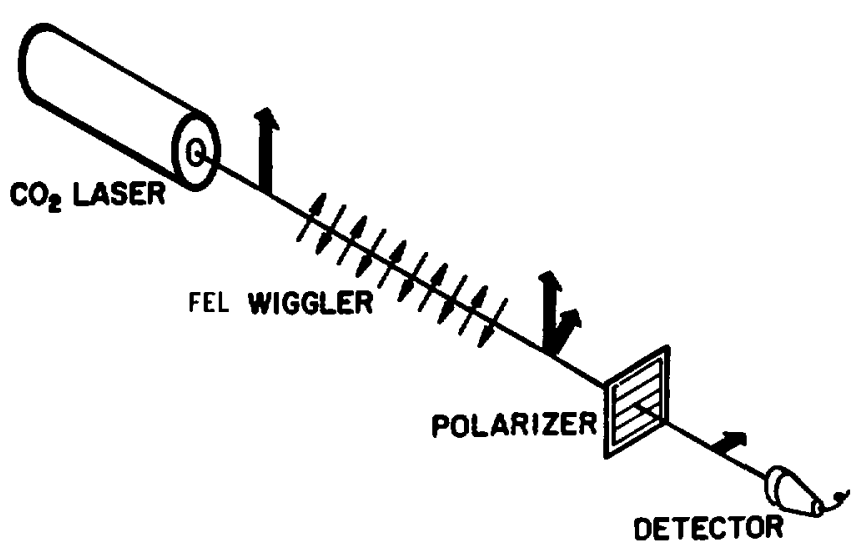

Fig. 22.

The gain experiment will be conducted using a pulsed, high-power $\mathrm{CO}_{2}$ laser at $10.6 \mu \mathrm{m}$. To measure the expected low gain, it will be necessary to suppress the incident $\mathrm{CO}_{2}$ laser by using crossed polarizers, with the free electron laser wiggler polarized at $45^{\circ}$. laser pulse. The energy gained and the location of the electron microbunches with respect to the laser and electron beam pulses are determined with a fast oscilloscope and pyroelectric detector. The width of the laser microbunches $c$ an be determined by measuring their spectral width $\left(\sim 1 \mathrm{~cm}^{-1}\right)$ with a monochromator. A. Accelerator Modifications As described above, at an electron energy of $20 \mathrm{MeV}$ and 25-A peak current during the micropulse, the inadequate shielding of the present enclosure dictates that we operate with a very short pulse (a few ns). This implies an accelerator operating in the stored energy mode. It is hoped that the electron gun pulse will be 3-4 ns long at the base and include 4 or 5 micropulses. To provide a fast rising, short pulse to the gun grid, a pulser will be constructed using an EG\&G design. The nominal jitter of the pulser is $40 \mathrm{ps.}$ The principal difficulty will be terminating the output from the pulser at the gun. At present, the $\mathrm{CO}_{2}$ laser pulse is expected to jitter 50- to 100-ns with respect to the trigger pulse. With additional effort (putting a Pockels cell in the laser cavity) the jitter may be reduced to $10 \mathrm{~ns}$ or less. This is still too large for solid synchronization with the electrci pulse. We plan to trigger the $\mathrm{CO}_{2}$ laser and sense the occurrence of the laser pulse with a pyroelectric detector. The laser pulse would be stored for about 50 ns with mirrors. During this time the electron gun pulse would be initiated so that the optical and electron pulses can arrive at the entrance to the wiggler within a nanosecond. In case the pyroelectric detectors ordered from LASL group E-10 are inadequate, the Pockels-cell pulse (about $14 \mathrm{kV}$ ) is available, if terminated properly. A portion of this pulse could be used to drive a diode laser, light pipe, and photodiode system that would trigger the electron gun pulse. The Pockels-cell pulse from the laser-triggered spark gap should be jitter-free with respect to the optical pulse. 
The micropulses will be accelerated without subharmonic bunching. This will alleviate added complexity in the injector. The accelerator structure will be of the side-coupled, standing-wave design presently being used, with matched coupling to the waveguide and klystron. Because of their high loaded

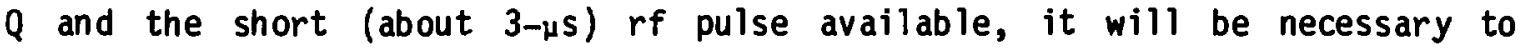
overdrive the accelerator tank with rf power to attain the desired fields in a sufficiently short time. An overdrive of about a factor of three for about one microsecond is anticipated, which is well within our rf-power capability.

Single-bunch beam loading in a single accelerator tank may limit the peak micropulse current for a $\pm 1 \%$ electron energy spread. Estimates based on the energy stored in the accelerator bore (1-in. diam) indicate that it may not be possible to achieve $25-A$ peak current with $\pm 1 \%$ electron energy spread. In fact, measurements on the 4-MeV linac indicate that the peak current may be limited to about one-half of the desired reak current to get $\pm 1 \%$ energy spread. This will not affect the energy extraction measurements obtained by dispersing the electrons in a magnetic field, but will reduce the laser gain in proportion to the current.

The beam emittance is another important quantity, as describer in Sec. 11, below. Crude emittance measurements on the existing 4-MeV linac indicate that $r_{\theta} \approx 10 \mathrm{~mm} \cdot \mathrm{mr}$ ad for $1.5-\mathrm{A}$ current averaged over the macropulse. The most optimistic emittance at $20 \mathrm{MeV}$ for the same current, is $\varepsilon \approx 2 \pi \mathrm{mm} \cdot \mathrm{mrad}$. A somewhat poorer emittance can be tolerated, but if the emittance is too large it should be possible to improve it by reducing the current. That may also be necessary to achieve the $\pm 1 \%$ energy spread, as noted above.

B. Beam Transport System

The FEL requires a small-diameter electron beain $(1.6 \mathrm{~mm})$ with a high micropulse current; thus, the transport system must not degrade the eiectron beam emittance nor, ideally, should it spread out the length of the micropulse. This requires the transport system to be both achromatic and isochronous.

The electron transport system is shown in Fig. 23. To minimize the beam aberrations, the wiggler is placed on the accelerator axis and the electron beam is bent off the axis and back on, to go around the mirror that brings the $\mathrm{CO}_{2}$ laser radiation into the wiggler. The dispersion introduced by the first bending magnet $c$ an be used to remove low-energy electrons from the beam by 


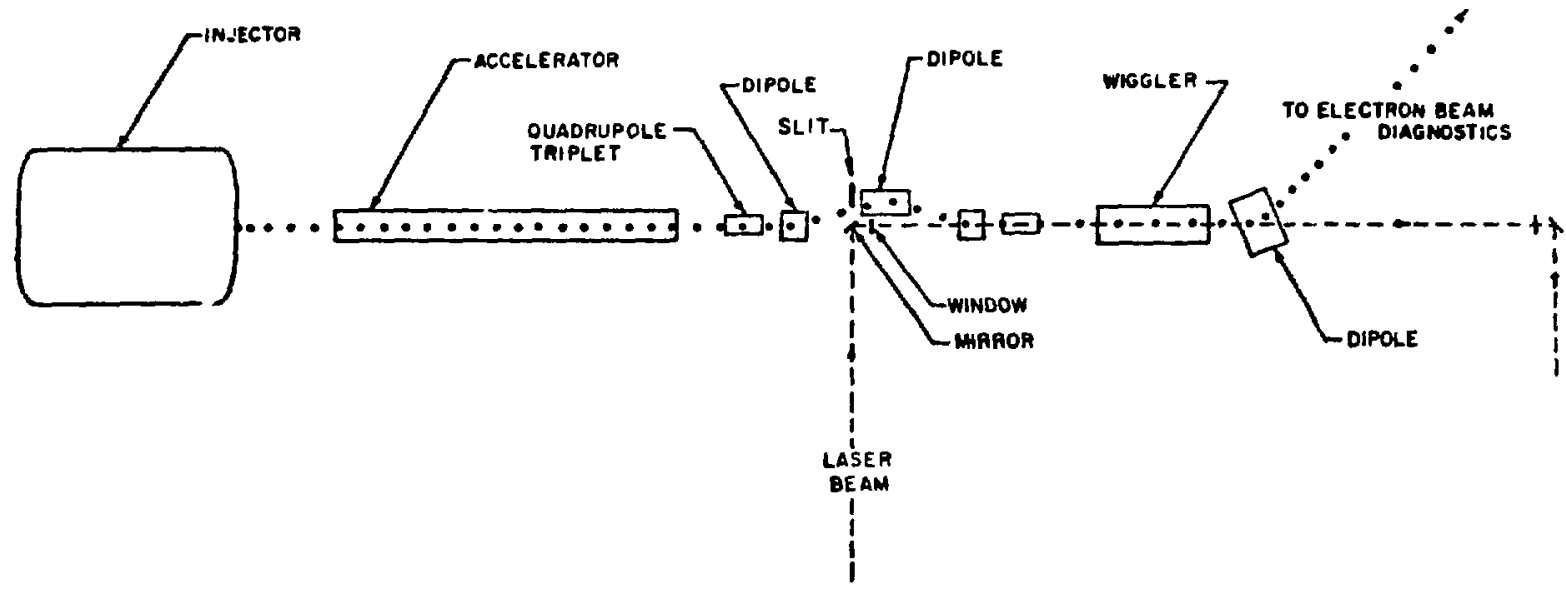

Fig. 23.

Electron transport system for FEL gain experiment.

placing slits at the second magnet, as shown. The quadrupole magnets focus the beam to the small diameter required by the FEL. The wiggler magnet provides a focusing effect on the beam in the vertical direction when the wiggler magnets are horizontal. Because the bending system focuses the beam differently in the vertical and horizontal directions, the wiggler magnets should be either horizontal or vertical and not at a $45^{\circ}$ angle to the horizontal plane. This difference in focusing in vertical and horizontal directions could be compensated by more quadrupole magnets, but not in the small space allowed.

In the vertical direction this transport system focuses the beam to a height of $1.6 \mathrm{~mm}$ at the entrance of the wiggler. The focusing effect of the wiggler then confines the beam to a height of $1.6 \mathrm{~mm}$ throughout the wiggler. This satisfies the requirements on electron beam diameter outlined in the previous section. In the horizontal direction the transport system focuses the beam to a width of $1.6 \mathrm{~mm}$ near the middle of the wiggler. The beam will have a width of about $3 \mathrm{~mm}$ at the entrance and exit. The only requirement on electron beam width in the horizontal direction is that the electron beam be with in the laser beam. This requirement is satisfied. of course, the size of the beam depends on the initial emittance of the beam from the accelerator. The emittance assumed for these calculations was $2 \pi m m-m r$. 


\section{Wiggler Design}

The wiggler magnet will be constructed of sintered $\mathrm{SmCO}_{5}$ permanent magnets precisely ground for correct orientation of the transverse magnetic field. By use of Hitachi Hicorex 96A or 21 material, which has a residual magnetic induction of approximately $10 \mathrm{kG}$, a magnetic field of $4 \mathrm{kG} c$ an be obtained in the central gap through which both the electrons and laser beam pass. The geometry of the wiggler is shown in Fig. 19. To simplify the construction and maximize the flexibility, the wiggler will be constructed of identical segments, except for the orientation of the residual fielo, with a uniform bore height. The periodicity will be varied by inserting nonmagnetic spacers between the magnetic segments. The field in the wiggler is given by the expression 55

$$
B^{\star} \cong i 2 \cos k_{w} z e^{-k_{w} h} B_{r} \frac{\sin (\pi / M)}{(\pi / M)} 1-e^{-k_{w} L} F,
$$

where $B=B_{x}+i B_{y}$ is the complex magnetic field, $k_{w}=2 \pi / \lambda_{w}$ is the wiggler wave number, $z=x+i y$ is the complex coordinate, $h$ is the halfaperture of the wiggler, $B_{r}$ is the remnant field of the magnetic material, $M$ is the number of magnet segments (top or bottom) per wiggler period, $L$ is the vertical dimension of a segment, and $F$ is the filling factor for the structure of magnetic segments and spacers. Exact computer codes are also available to compute the magnetic field. 55,56 Because of this compromise with practical considerations, it will not be possible to build a fully optimized wiggler, in the sense of Sec. II, above. However, near an optimum, the performance is not sensitive to variations in the parameters. Thus, good extraction efficiency will be achieved, together with a double-peaked distribution function, like that shown in Fig. 15, characteristic of a tapered wiggler.

The wiggler will be placed in a section of the evacuated electron beam line, as shown in Fig. 23. As discussed above, it should not be necessary to focus or constrain the electrons along the wiggler axis with in the wiggler itseif. The natural focusing properties of the wiggler will constrain the electrons in the vertical directirn, and the electrons need only (and will) stay with in the laser beam in the horizontal direction. 


\section{Laser Oscillator and Amplifier}

The $\mathrm{CO}_{2}$ laser oscillator is an advanced design borrowed from LASL's Antares fusion laser research. 57,58 It features a nominal $0.25-J$ output in a 1 -ns pulse at a repetition rate of $1 / 3$ to $1 \mathrm{~Hz}$. It operates in a single longitudinal mode and on a single line (tunable) by use of a low-pressure smoothing tube and grating, respectively. In the far field, a nearly Gaussian spatial profile (Strehl ratio of 0.5 ) is obtained. The schematic of this oscillator shown in Fig. 24 illustrates the four-pass reinjection scheme by which such intense and short laser pulses are produced. The 1-ns pulse is sliced out of the gain-switched oscillator output pulse (40-ns FWH) by a CdTe Pockels-cell/germanium stacked-plate-polarizer arrangement triggered by a spark gap. This system (Fig. 25) can produce pulses of $0.5-n s$ duration and

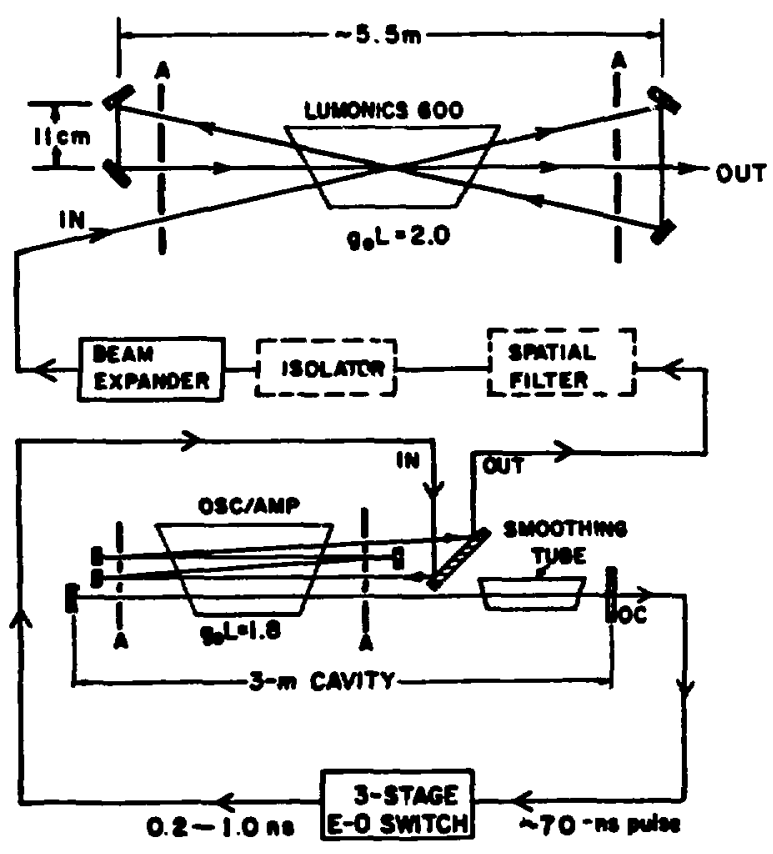

Fig. 24.

Schematic of the three-stage $\mathrm{CO}_{2}$ laser systen, showing the highpressure reinjection oscillator, the electro-optic switch and the triplepassed TEA Lumonics power amplifier.

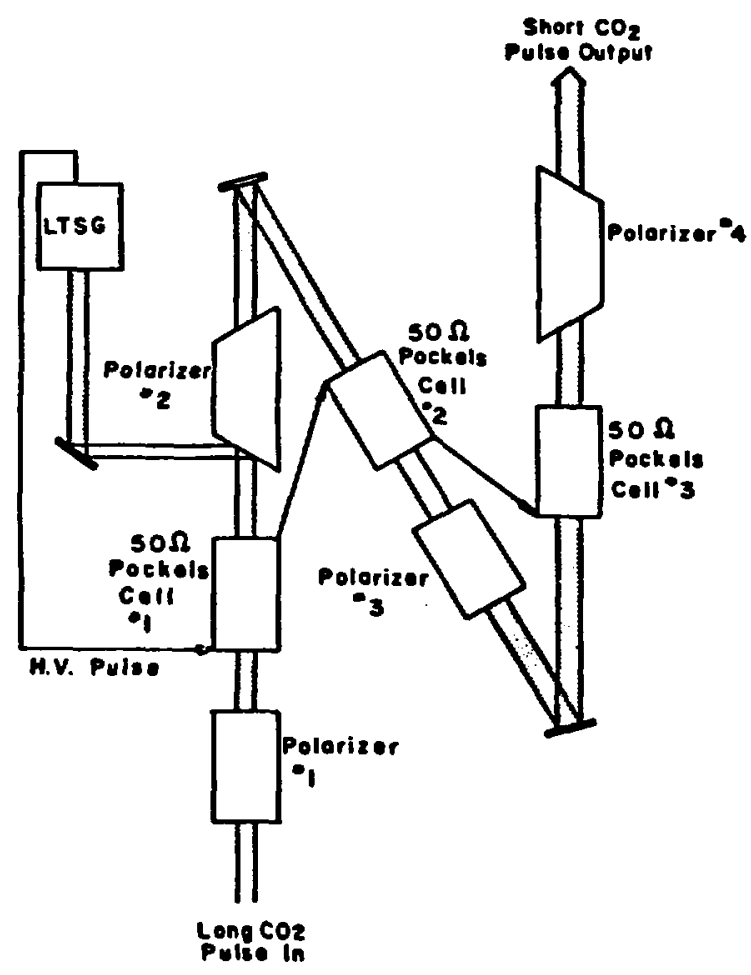

Fig. 25.

Conceptual design of the 3-stage Pockels-cell switch. The lasertriggered spark gap is activated by the leading edge of the long 40 - to 70-ns) pulse, which, having the crossed polarization, is reflected from polarizer \# 2. 
longer. The switched-out pulse is then expanded and directed through a Lumonics $600 \mathrm{~A}$ amplifier $(0.04 / \mathrm{cm}$ weak signal gain over a $9-\mathrm{cm}$ diam.) that can be pulsed at $1 / 6 \mathrm{~Hz}$. The resultant energy in a 1-ns pulse is several joules or more if a triple-pass scheme is used.

Spatial overlap of the laser and electron beams will be accomplished by measuring the transmissions of electrons and laser beams individually through apertures centered on the wiggler axis. The apertures, located at the entrance and exit of the wiggler, will be opened or removed during the actual gain experiments. In addition, a minimal amount of beam wander of the $\mathrm{CO}_{2}$ laser pulse will be tolerable. In a similar laser system presently used in fusion target experiments at LASL, negligible motion of the beam has been detected.
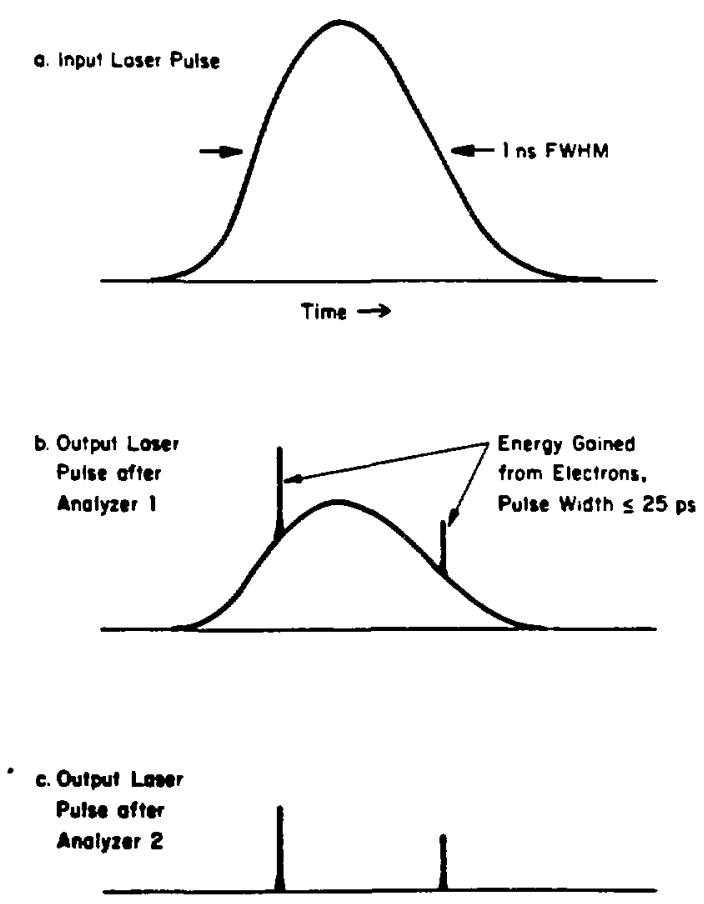

Fig. 26 .

Method of measuring the $\mathrm{CO}_{2}$ laser inputer intensities associated with energy gained from the electron bunches. The temporal iocation of the stimulated pulses within the 1-ns laser pulse and the relative energy in the two pulses is measured after Analyzer 1. The total energy contained in the two pulses is measured with a pyroelectric calorimeter after Analyzer 2.

\section{E. Laser Diagnostics}

To insure laser and electron beam coincidence in time, a sufficiently long laser pulse ( $\sim$ ins, nominally) will overlap two or more electron bunches that are separated in time by $0.75 \mathrm{~ns}$, as shown in Fig. 26. The temporal location of the bunches with in the laser pulse will be evident as energy gain at $10.6 \mu \mathrm{m}$ superposed on the laser pulse waveform at the exit of the wiggler.

The net optical pulseform transmitted through a nearly crossed polarizer (suppressing most of the $\mathrm{CO}_{2}$ input polarization) would appear as in Fig. 26b on ar infinitely fast detector-oscilloscope monitor. A sufficiently fast oscilloscope will provide a useful bandwidth-limited version of the pulseform to allow location of the electron pulses.

The necessary optical diagnostics and their deployment about the magnetic wiggler are indicated 


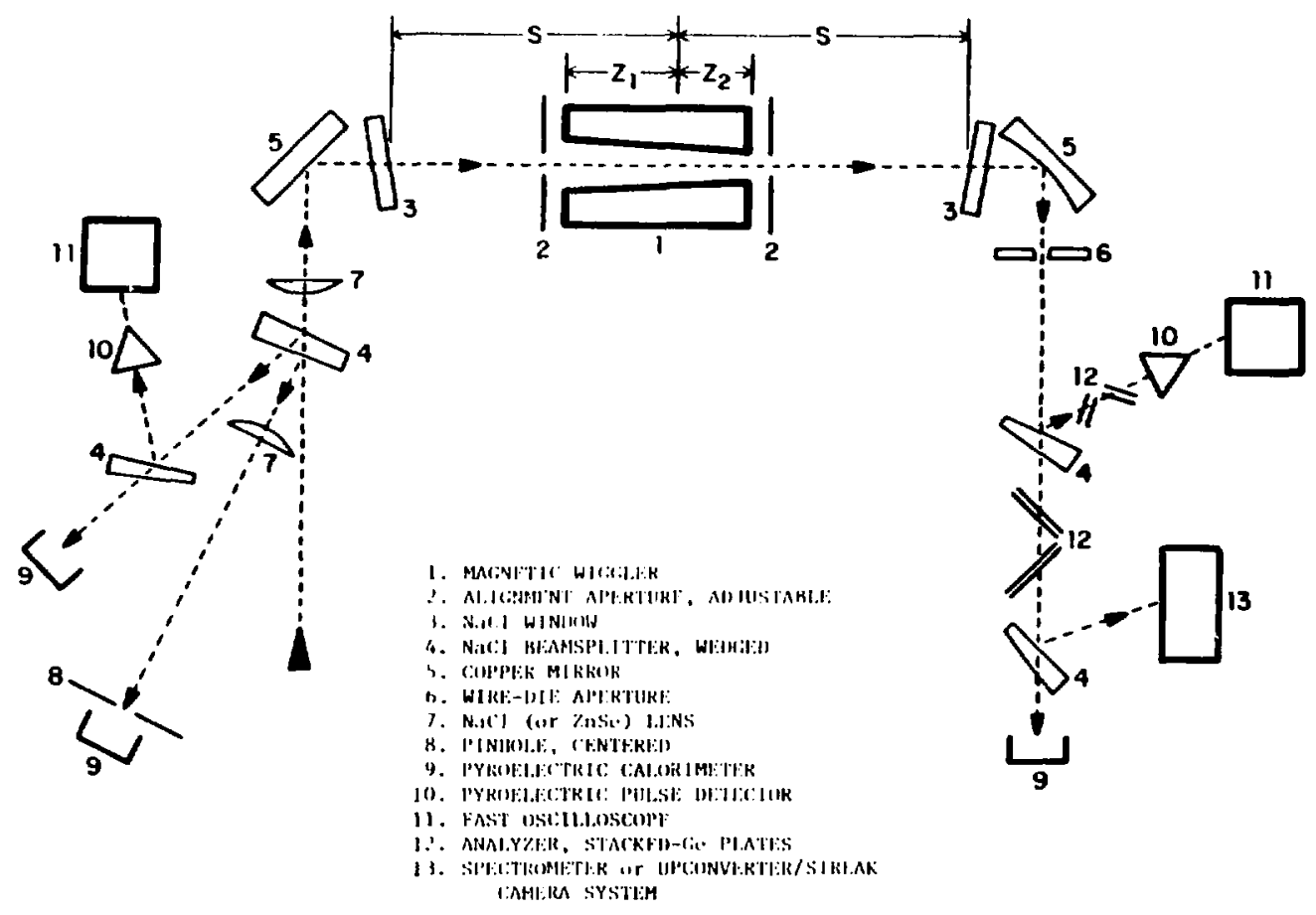

Fig. 27.

Schematic of the optical diagnostics for the 10.6- $\mu \mathrm{m}$ FEL gain experiment.

schematically in Fig. 27. Energy, and temporal and spatial waveforms of the $\mathrm{CO}_{2}$ laser radiation are to be measured. At the input, the total energy will be measured with a calorimeter and/or a pyroelectric ratiometer detector. The temporal shape of the pulse will be measured with a fast pyroelectric detector and fast oscilloscope (Tektronix 519 or LASL 5-GHz model). The beam profile at the focus within the wiggler will be monitored by use of a duplicate lens that focuses energy split-off of an $\mathrm{NaCl}$ beamsplitter. A pinhole located at the focal plane and centered on the peak of the Gaussian-shaped spatial profile transmits energy to a pyroelectric detector. The ratio of this energy to the total input energy provides a monitor of the shot-to-shot stability of the focused spot intensity as well as a calibration of the intensity at the focus within the wiggler. The pinhole will also be used to scan the laser beam to measure the spatial profile.

At the output, an aperture passes only the central most intense portion of the beam so as to avoid integrating the observed gain over the spatial intensity profile. A spherical copper mirror collimates the output through 
germanium stacked-plate analyzers that are crossed, with respect to the input $\mathrm{CO}_{2}$ laser polarization. The first analyzer transmits a small fraction of the intput energy. A portion of this is reflected to a fast detector and uscilloscope for determining the laser intensities corresponding to the observed energy gained from the electron bunches. The second polarizer essentially quenches any of the remaining $\mathrm{CO}_{2}$ pulse and transmits only the energy gained from the electrons as indicated in Figure 26c. The total energy in these pulses is measured with a pyroelectric detector and ratiometer readout for comparison with the input energy. This detector (Laser Precision) is sensitive to less than $1 \mu \mathrm{J}$ and is unaffected by the accelerator radiation environment. The extinction ratio of the second polarizer can be set at 3000:1 with a commercial design and to $10^{5}$ with a LASL design. The relative amount of energy in each of the transmitted pulses representing energy from the electrons will be measured by capacitive integration at the input of a standard oscilloscope. The heights of the spikes on the oscilloscope display are proportional to the energy in the short ( -25 ps) pulses. Comparison with the total energy measurement provides the separate energy in each pulse.

A first-order measurement of the pulsewidth of the picosecond pulses will be accomplished by a spectral bandwidth measurement with a grating spectrometer using a pyroelectric array or ir vidicon at the output. For better resolution, a Fabry-Perot interferometer may be used. From the spectral bandwidth, the temporal pulsewidth may be inferred by Fourier transform and assuming a Gaussian profile.

If time allows, a direct and linear measurement of the pulse width and temporal profile of these short pulses will be obtained with a streak camera having an S-1 spectral response. ip-conversion of the 10.6- $\mu \mathrm{m}$ laser radiation with an auxiliary 1.06- $\mu \mathrm{m}$ beam coincident in a Proustite mixing crystal will provide $0.96-\mu \mathrm{m}$ radiation for wich the $\mathrm{S}-1$ phosphor streak tube is sensitive. The output from the camera will be immediately read out by use of an intensified SIT detector and digital read-out (Optical Multichannel Analyzer) and displayed on an oscilloscope and computer printout.

Another primary measurement, which supplements the optical measurements of energy gained from the electrons, is the measurement of the momentum distribution of the electrons exiting the wiggler. The captured electrons will have lost energy to the laser beam, and the amount of energy lost will be measured by dispersing the output electron beam with a magnet and detecting 
the location and intensity of the fluorescence the electrons cause on a fused silica target. The linear separation and relative fraction of trapped and untrapped electrons is measured with a vidicon. The expected output will reflect the double-peaked distribution function characteristic of tapered wigglers, like that shown in Fig. 15.

All of the signals from the above diagnostics will be fed to an on-line minicomputer for processing, and the immediate print-out will be essential for real-time adjustments in the accelerator and/or laser output, alignment, and synchronization to maximize the gain.

F. Impact of Optical Damage on the Design

The damage resistance of the optical elements shown in Fig. 28 determines the dimensional scaling of the gain experiment. ${ }^{59}$ We have calculated the size and spacing of the optics for the $0.92-\mathrm{m}$ wiggler design whose parameters are given in Table IV. For a $1.5 \mathrm{GW}$ pulse of 1-ns duration (FWHA), the separation $S$ of the input and output $\mathrm{NaCl}$ windows is set at $2.8 \mathrm{~m}$, at which distance the diameter of the focused laser beam is large enough to obtain a peak energy density of less than $2 \mathrm{~J} / \mathrm{cm}^{2}$. This energy fluence is considered safe for multishot operation and was determined from the results of a 100-shot damage experiment shown in Fig. 28. A safe operating level of $50 \%$

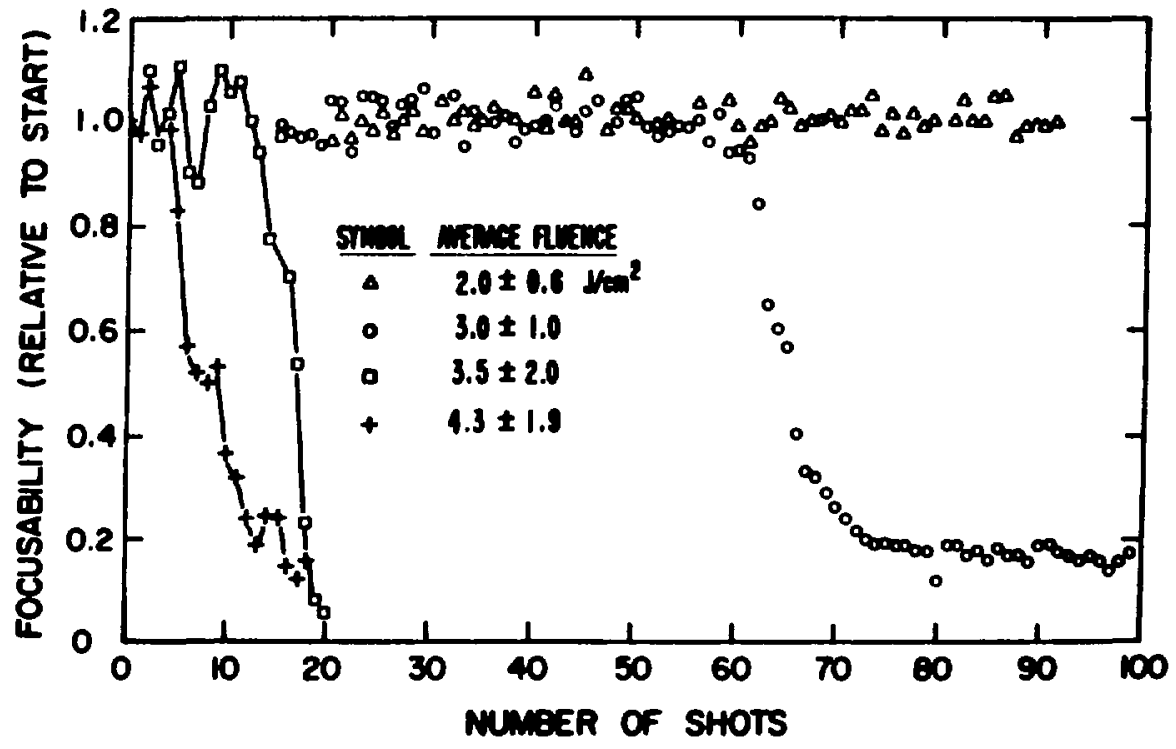

Fig. 28.

Focusability versus number of $\mathrm{CO}_{2}$ laser pulses through an MaF AR-coated $\mathrm{MaCl}$ window. Damage occurred on the exit surface. Pulsewidth was 1.2 ns and spot-size radius was $1.1 \mathrm{~mm}$. 
of the single-shot damage threshold is indicated. Similar calculations were performed to determine the minimum allowable focal length and diameter of the ZnSe (AR-coated) input lens and the minimum beam size incident upon the germanium stacked-plate analyzers. A multishot operating fluence of $0.75 \mathrm{~J} / \mathrm{cm}^{2}$ was used for the lens (see Fig. 29) and $1 \mathrm{~J} / \mathrm{cm}^{2}$ was determined to be a safe level for germanium at Brewster's angle. The damage resistance of polished copper mirrors at $45^{\circ},>8 \mathrm{~J} / \mathrm{cm}^{2}$, offered no additional design constraint.

G. Schedule

A brief chart showing the schedule for the experiment is shown in Fig. 30. The critical path leads through the accelerator rf-cavity structure, testing the beam transport, and integrating the system. Building and tuning the structure takes time, but it is straightforward and has been done many times before at LASL. The transport system will be tested and adjusted as soon as the beam is available from the accelerator, and the system integrated as soon as the $\mathrm{CO}_{2}$ laser is available. This is paced by the delivery of the final amplifier section, a commercial unit (appropriately modified).

IV. CONCLUSIONS AND RECOMMENDATIONS

The results of the effort described in this report continue to support the conclusion that a high-average-power $(>100 \mathrm{~kW})$, high-efficiency $(>25 \%$ overall), short-wavelength $(0.5-2 ? \mathrm{~m}$, tunable) FEL system is possible with

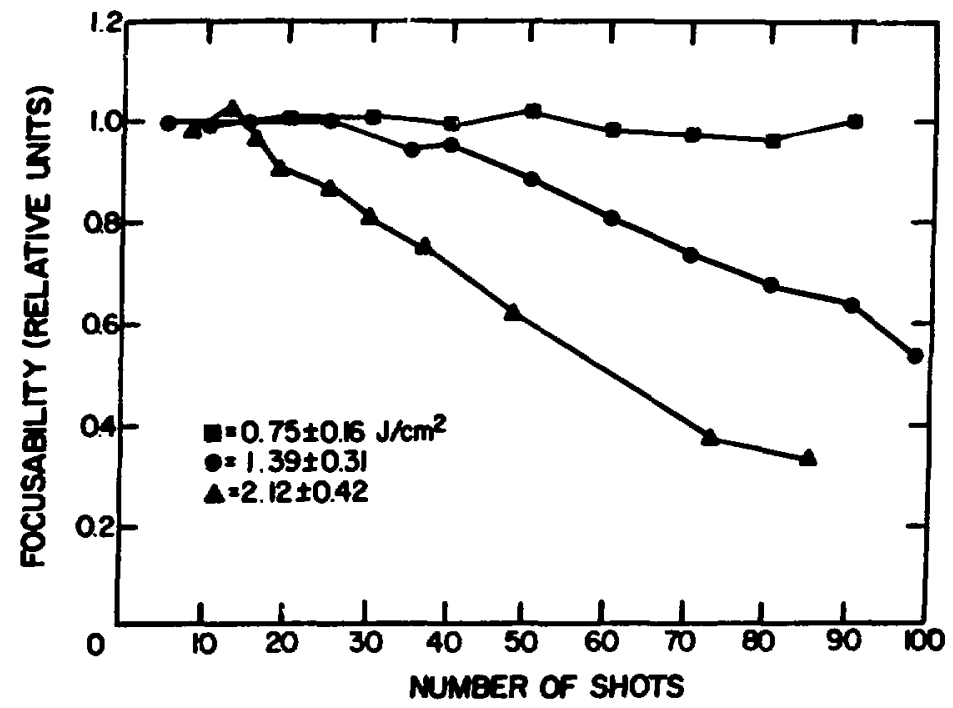

Fig. 29.

Focusability versus number of $\mathrm{CO}_{2}$ laser pulses transmitted through an AR-coated ZnSe window. Same conditions as Fig. 28. 


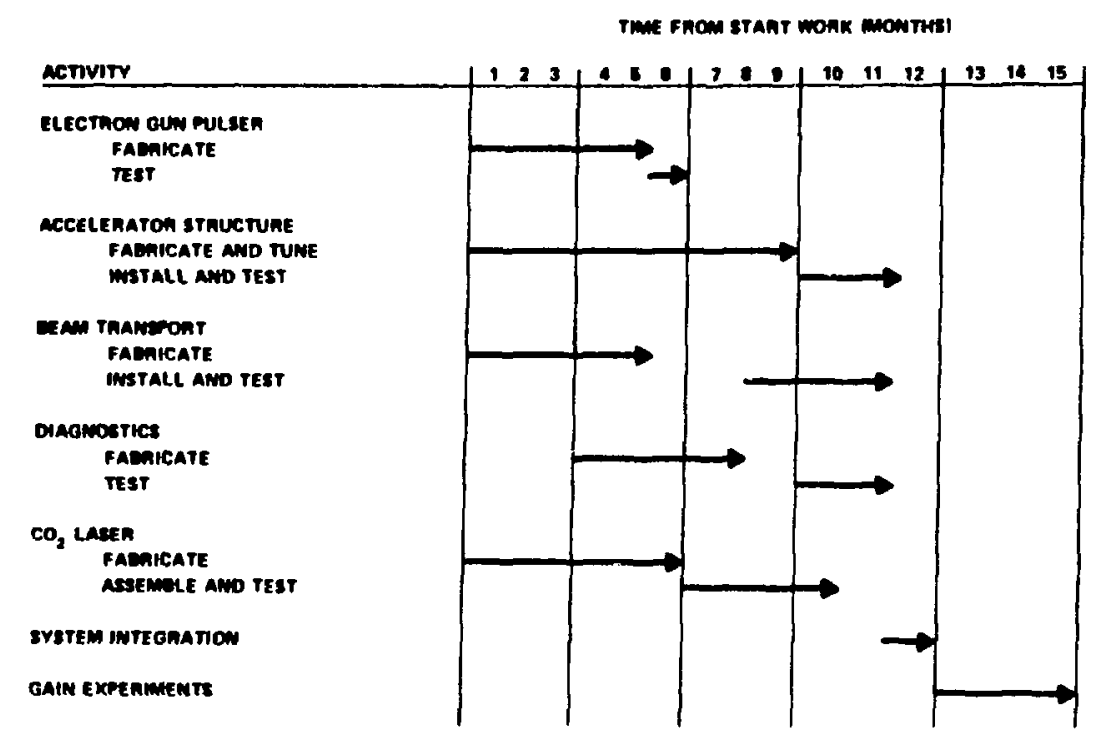

Fig. 30 .

Preliminary schedule for free electron laser experiment.

only modest developments from existing technology. The best technological approach for achieving such performance at wavelengths in this $r$ ange is to use a racetrack-type $r f$-accelerator/decelerator system. In this approach, an rf accelerator accelerates the electron beam to high energy for injection into the FEL. The electrons from the FEL are then brought around and decelerated in the same accelerator structure to recover their remaining energy. Although the design studied at Los Alamos has a nominal 100-kW average power, this does not represent a limit, and higher powers should be possible. Wavelengths shorter inan $0.5 \mathrm{\mu m}$ should be possible also, but to extend the $h$ igh power and high efficiency to shorter wavelengths will require the development of injectors with lower emittance than presently available.

Although no inventions are needed to achieve this level of performance, development will be required in several areas. The most critical requirement is to demonstrate experimentally that high extraction efficiency can be achieved by means of tapered wiggler magnets. Accordingly, the principal effort under the preseni program has been to design an experiment for just this purpose. The second most critical requirement is to demonstrate experimentaliy that an $r f$ accelerator can be used simultaneously to decelerate and recover the energy from the electron beam emerging from a FEL. A smallscale experiment to do this should be given very high priority. Design of the 
optical cavity should be given very high priority and should be given more careful attention than has been possible so far. The design used in the present study by LASL is simple but awkward, and only a cursory analys is of the thermal distortion has been made. However, the design is very conservative and the optical system should not offer any fundamental obstacles to high-power FEL development. Finally, several aspects of accelerator technology require further development. Better injectors should be developed to provide lower emittance for shorter wavelengths, and higher peak currents for higher gain and extraction efficiency. Also, development of the rf cavities in the accelerator should be undertaken to suppress instabilities. These instabilities appear in the form of higher cavity modes that could cause beam break-up or reduce the accelerator efficiency. Such instabilities represent an important concern in machines with high beam loading, but it should be possible to suppress them with known techniques.

The theoretical work undertaken in the present program has provided both more accurate theories than were available before and new capabilities in terms of coherent effects and pulse propagation, two-dimensional effects and plasma effects. For the parameter space of interest to the present program, the new theoretical results agree with earlier results, as far as those theories could go. The first use of the new theoretical capabilities was to help design and predict the performance of the high-power laser and the 10-um demonstration experiment. The next task of these new theories therefore should be to explore those aspects that could not be described adequately by earlier theories. For the parameter space of interest to this program, the important effects include propagation and evolution of short laser pulses (microbunches) in the pulsed gain medium from an $r f$ linac, and parasitic instabilities (Raman sidebands) related to excitation of coherent synchrotron oscillations. The existing codes should be extended to provide a better description of the degradation of laser performance caused by the transverse momentum spread and finite diameter of the electron beam. Two-dimensional effects and plasma effects are most important in systems operated with high-intensity, low-energy electron beams, such as those being developed at MRL and Columbia University. Therefore, it is recommended that the numerical particle-beam codes developed at LASL be applied to these systems. This is especially true because oscillation in these short-pulse, high-gain systems is growing from noise during most of the pulse. This very important phenomenon is difficult to treat by any means other than 
the LASL particle-beam code. In addition to the highly accurate theories described above, a simple theory has been developed to study the optimization and scaling of FELs. The theory shows that for an optimized laser, the extraction efficiency varies roughly with the square root of the laser power in the cavity, and that gigawatt laser powers are required to exceed a few per cent extraction. The simple theory also considers the effects of transverse momentum and radial extent of the electron beam on laser performance. The results show that the shortest wavelength achievable with high efficiency is proportional to the electron beam emittance. It should be possible to build lasers at wavelengths below about 0.5-1.0 $\mu \mathrm{m}$ with emittances achieved in existing linacs, but better injectors will have to be developed to reach shorier wavelengths. Because emittance becomes such an important effect at short wavelengths it is important to develop a more accurate theory to describe transverse effects, and to understand better the origin and cure of emittance growth in $r f$ linacs. The origin of the emittance in existing linacs apparently is not due simply to the temperature of the cathode. If this were the case, much smaller emittances would be possible.

The conceptual design of the experiment to demonstrate high extraction efficiency has been completed. The experiment will make use of an existing linac-suitably modified--to demonstrate approximately $5 \%$ extraction efficiency from a $20-\mathrm{MeV}$ beam in a $1-\mathrm{m}-10 n g$ wiggler operating at $10 \mu \mathrm{m}$. To minimize the radiation from the electron beam, the linac will be operated in 5-ns bursts. About $1.5 \mathrm{GW}$ of $10-\mu \mathrm{m}$ radiation from a pulsed $\mathrm{CO}_{2}$ laser will be required to trap and decelerate the electrons. In addition to laser gain, the energy extracted from the electrons will be measured by dispersing them in a magnetic field to analyze their momentum distribution. The linac modifications are reasonably straightforward, but will require about ten months to complete. The FEL experiment is complicated by the strict alignment and symchronism requirements, and by the small (1-2\%) laser gain that is expected. Solutions to these problems are believed to be well enough in hand so that a one-year time scale for obtaining the first experimental results seems realistic.

Because of the potential importance of pulse propagation effects for the short ( $-25 \mathrm{ps)}$ laser and gain (electron) bunches in an oscillator configuration, it is important that the gain experiment be followed as soon as possible by an oscillator experiment. Such an experiment will also make it possible to 
study start-up in an oscillator with a tapered wiggler, as well as the potentially deleterious effects of coherent synchrotron Raman instabilities. Because of the urgency of such an experiment, it is important to begin work on it during the coming year, if possible.

Finally, it is important to demonstrate deceleration and energy recovery from the electrons emerging from the wiggler. Although electrons have been decelerated in other systems, such as the Reflextron at Chalk River, problems may appear in highly beam-loaded accelerators such as those proposed for FELs. In the oscillator configuration the smal1, 10-4m, FEL experiment discussed here would provide an ideal test bed for demonstrating energy recovery, and it is strongly recomended that such an experiment be undertaken as soon as possible. 


\section{INTRODUCTION}

The PIGMI Program of the AT Division at LASL, supported by the National Cancer Institute, Division of Research Resources and Centers, Department of Health, Education, and Welfare, has completed three and a half years of a project to develop the technology for the optimal pion generator for medical irradiations (PIGMI). The program is progressing we?l and is designed to converge during the next year into a rather detailed description of the technologies required to build a PIGMI.

Some of activities reported here were partially supported by other budgets in the sense that the work was also applicable to PIGMI. In particular, the RFQ development was supported primarily by the FMIT program, OFE, DER, DOE. In return, some direct PIGMI work benefits other programs of the AT Division.

The PIGMI program's major accomplishments during the report period include completion of the injector beam measurements, completion of the 440-MHz rf power source, completion of the instrumentation-and-control system, assembly and test of APF accelerator section, installation of low- and high-energy beam transport systems, development of RFQ beam-dynamics program, PARMTEQ, design, fabrication, assembly, and test of the RFQ accelerator, final decision on low-energy configuration for PIGMI, assembly of the DTL section of PIGMI Protoype, completion of sample set of PM quadrupoles, optimization of the DAW cavity geometry, fabrication of model cavities of DAW, final decision on DAW support geometry, acquisition of additional laboratory space for DAW power test, partial assembly of 1320-MHz rf power source for DAW test, and pion channel design studies.

\section{INJECTOR}

The injector continued to work well throughout the year, but powerline transients and electromagnetic radiation generated during the sparkdowns continued to interrupt the control system and interfere with beam measurements. Special care was taken to isolate the high-voltage system and to 
provide a single-point ground. In addition, the entire injector was enclosed in a Faraday cage. These measures helped significantly; however, one additional modification has essentially eliminated sparkdowns and their associated problems. All of the utility and instrumentation cables connecting the ion source to the high-voltage dome were wrapped around an insulated cylinder, adding inductance to the path over which arcdown currents must flow. This limits to a sufficient degree the instantaneous energy dissipated during an arcdown, thereby greatly reducing the amount of radiated noise. This modification has reduced by about $90 \%$ the frequency of arcdowns by apparently quenching sparks in their early stages of formation.

Two new pieces of equipment, designed and implemented in the injector, have significantly improved the ion source operation. The new master timer provides a locked sequence of instrumentation signals to synchronize the operation of the ion source, the $r f$, and any diagnostic measurements. All of the necessary variables, including pulse widths, delays, and frequencies are adjustable. The arc-pulse signal is transmitted to the high-voltage dome through a fiber-optic link. This signal triggers a new arc-pulse power supply that generates a very sharp flat arc pulse. This new supply eliminates the need for an arc starter and delivers a pulse whose shape is independent of length and repetition rate.

The brightness of the beam was improved with the installation of a boron nitride collar in the plasma expansion cup. To reduce the erosion observed in the anode aperture, which is caused by backstreaming electrons, a smal1, permanent-magnet electron trap was installed just downstream of the ground electrode. Because of the instrumentation problems associated with sparkdowns, the plan to provide remote control of the ion source, with a microprocessor at high voltage, was abandoned. The few necessary adjustments are easily made with a lucite rod from outside the Faraday cage.

Following a program of measurements to characterize the operation of the alternating phase focusing (APF) accelerator, the accelerating column was modified to a two-gap configuration to deliver about $40 \mathrm{~mA}$ at $100 \mathrm{keV}$ for injection into the radio-frequency quadrupole (RFQ) test accelerator. Likewise, the low-energy transport system was modified to deliver a matched beam to the RFQ. Ouring each of these phases the operation of the injector and low-energy transport systems were fully studied. Emittance measurements 
of the beams emerging from the dc column were very close to those expected from numerical calculations and indicate an exceptionally high brightness. Beam-profile measurements made downstream of the solenoid indicated a slight misalignment between the source and the solenoid. Because each device was aligned to within a reasonable tolerance, steering magnets were designed, fabricated, and installed on the beam line; this has solved the problem. A second emittance measurement made downstream of the solenoid showed that for the 100-keV beam, spherical aberrations were causing distinct degradation in the transverse properties of the beam. This would only be correctible with a new larger-aperture solenoid.

For both accelerator tests the transport design was confirmed by on-line emittance and profile measurements. Numerical fits to the experimental data were used in the TRACE code to represent beam sizes and emittance orientation. By allowing for space-charge neutralization, agreement between measurements and calculation was easily demonstrated.

\section{III. $r f$ POWER SOURCE}

The $r f$ power system, including a 440-MHz klystron, modulator, and associated amplifiers and controls, worked well throughout the tests of both the APF and RFQ structures. The APF cavity required about $850 \mathrm{~kW}$ of pulsed power, whereas the RFQ required about $300 \mathrm{~kW}$. Each test was carried out over a period of several months and the power stand performed admirably. A closedloop frequency-control unit was installed for the RFQ tests, which caused the $k$ lystron to track in frequency for variations in cavity temperature. This has all but eliminated the necessity for adjusting the rf during accelerator runs.

\section{INSTRUMENTATION AND CONTROL SYSTEM}

In support of accelerator studies, a complete complement of computerbased bean-diagnostic measurements was implemented. The beam line equipment provided the following measurements and controt.

1. Emittance measurements,

2. harp-and-wire scan profile measurements,

3. faraday-cup beam-current measurements,

4. bean-current transformer measurements,

5. direct viewing of beam spot size,

6. momentum analysis, 
7. discriminator-foil energy analysis,

8. four-jaw aperturing, and

9. horizontal and vertical steering.

All of the above equipment, much of which is shown in Fig. 31, is mounted on pneumatic or stepping-motor actuators, and is operated under computer control from an operator's console. A large package of software has been written and implemented that supports a wide variety of beam and accelerator measurements and data analyses. The entire system has been reliable, responsive, and easy to use.

During the tests of the APF structure, the accelerated beam was dumped in a special Faraday cup mounted inside a dummy drift tube at the interface between the APF cavity and the high field gradient test cavity (PIGLET) through which the power was provided. This special Faraday cup was fitted with an energy-discriminating foil window. High-energy electrons were magnetically suppressed, whereas secondaries originating in the copper were electrically suppressed. The cup therefore provided a measure of accelerated beam current only.

During tests of the RFQ structure, the PIGLET cavity again was used to supply rf power to the accelerating structure; however, this time in a coaxial manifold mode. In this case, the accelerated beam that emerged from the structure was expected to be small and bright in emittance, at an energy of only $640 \mathrm{keV}$. A transport system was designed and assembled from available components, which would allow both emittance and momentum measurements. The system includes a quadrupole doublet, a $45^{\circ}$ analyzing magnet, beam-diagnostic boxes, beam dump, and vacuum system.

\section{APF ACCELERATOR PROTOTYPE}

All four PIGMI prototype tanks were delivered. The first section, the APF, has been loaded with drift tubes and tested under power with beam. The APF tank contains 28 drift tubes, each with a unique length. Each drift tube was fabricated from mild steel and fitted with three hollow mounting stems for support and to provide water cooling. Following an initial copperplating, all drift tubes were mounted in the tank and aligned. Frequency measurements indicated that a thicker plating was required. Two subsequent plating jobs were required to bring the assembly into resonance at the proper frequency. 


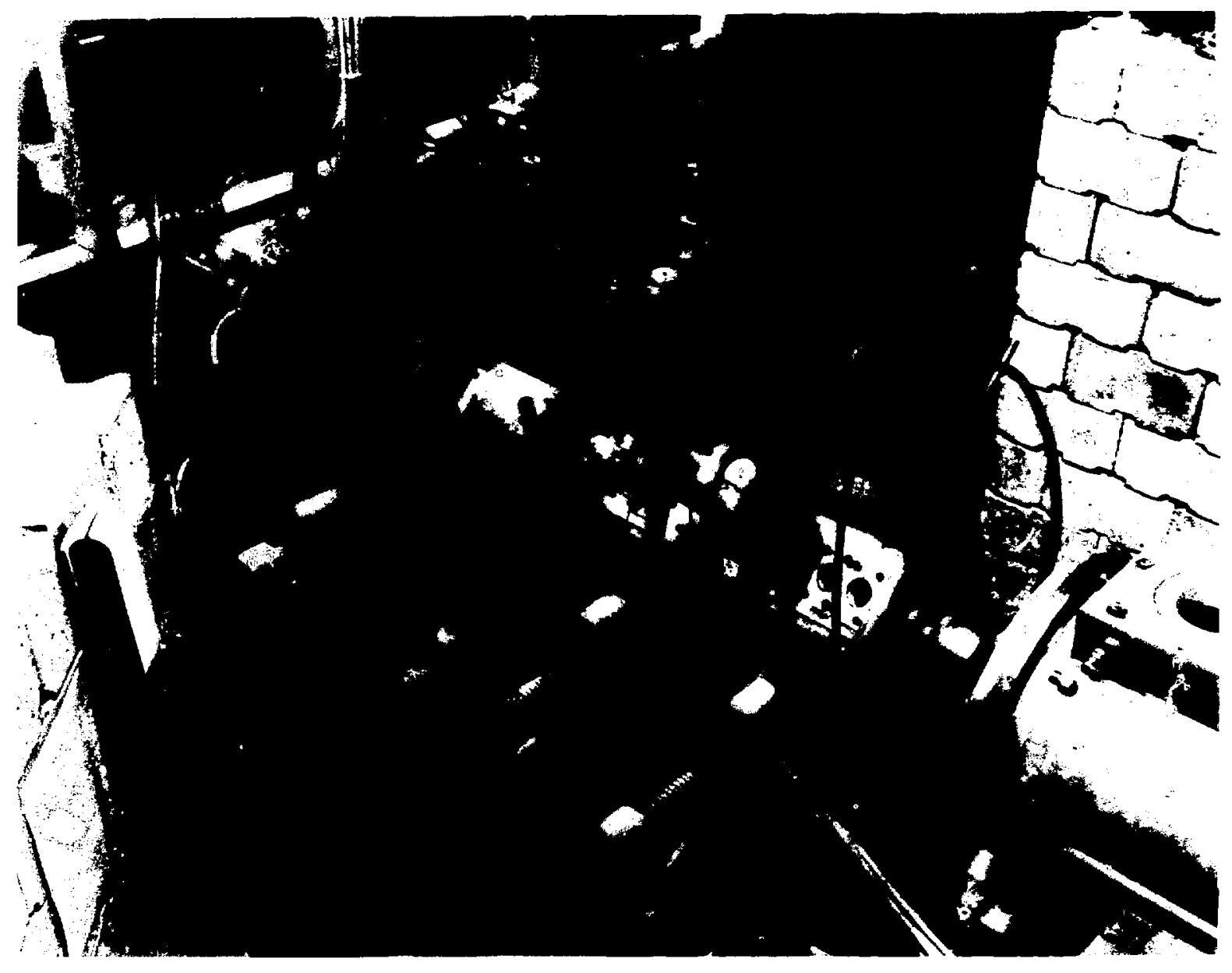

Fig. 31 .

PIGMI diagnostic equipment. 
Each drift tube was aligned transversely in a conventional way using a transit and a target inserted in the bore of each drift tube. A special depth guage was developed for measuring the longitudinal positioning of each drift tube. Working on the principle of electrical continuity, the guage did not require a solid mechanical contact, thereby minimizing the drift-tube deflection during the measurement of its position. Stems were manually bent to correct the longitudinal position of the drift tubes.

The APF tank was bolted to an adapter ring mounted on the upstream end of the PIGLET high-power test cavity, using elastimer 0 -rings for vacuum, and gold wire for electrical integrity. Power was coupled into the APF cavity from PIGLET, used primarily because of its existing rf drive configuration and rf window. A special dumy drift tube was fabricated to provide a match between the two dissimilar cavities. Bead pulls indicated flat fields in the APF structure, but somewhat higher fields in the gap between the final APF drift tube and the first dumm drift tube. The design fields were ultimately exceeded by about $10 \%$, but the field level was constrained by this gap.

Initial operation of the APF structure was plagued by vacuum difficulties associated with water leaks in the stem-to-drift-tube body joints. This problem, associated with long-term crevice corrosion of the braze material because of incomplete washing after copperplating operations, was extremely elusive and is detailed in a separate series of memos. Leaks were plugged using a commercial epoxy-base preparation designed for such applications. To further avoid such problems, the tank was operated at reduced duty using air cooling only.

The APF linac structure was developed for the acceleration and focusing role in the low-beta portion of PIGMI. In this structure, the transverse, as well as the longitudinal, focusing forces are produced by the rf electric fields. By arranging the drift-tube lengths in a conventional standing-wave drift-tube linac (DTL) structure, the gaps can be positioned to provide acceleration, as well as a succession of focusing and defocusing forces. These forces result in satisfactory cortainment of the beam in the six-dimensional phase space without requiring additional focusing fields. 


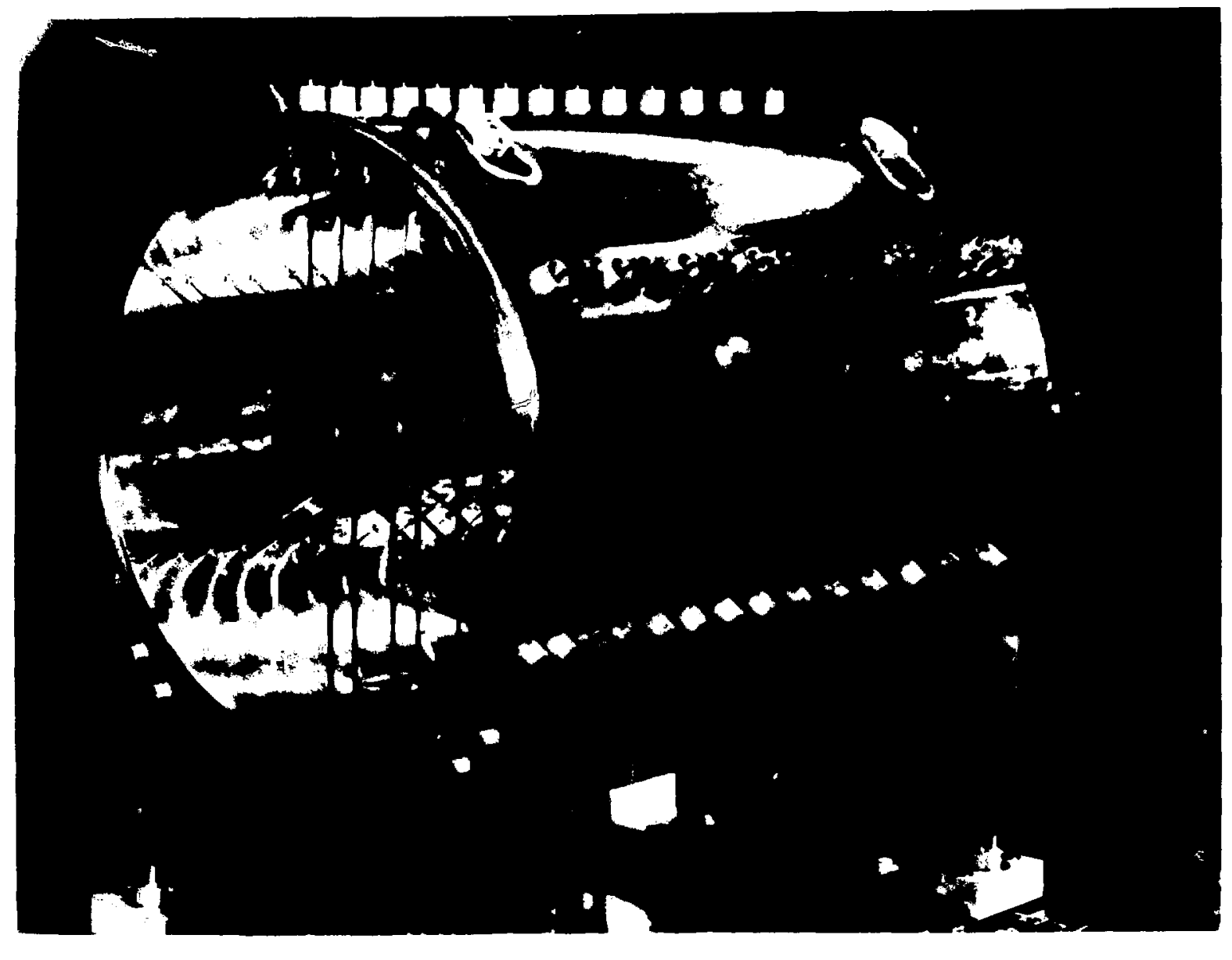

Fig. 32. APF Cavity.

Figure 32 shows the APF portion of the PIGMI prototype. It is $63 \mathrm{~cm}$ long, $46 \mathrm{~cm}$ in diameter, and contains $28 \mathrm{drift}$ tubes, none of which contain quadrupole lenses. The drift-tube bodies vary in length from a minimum of $0.690 \mathrm{~cm}$ to a maximum of $3.003 \mathrm{~cm}$, and are $8 \mathrm{~cm}$ in diameter. The bore-hole radius increases from $0.402 \mathrm{~cm}$ in the first drift tube to $0.487 \mathrm{~cm}$ in the 28th drift tube. The APF operates at $450 \mathrm{MHz}$ and accelerates a proton beam from $250 \mathrm{keV}$ to $1.011 \mathrm{MeV}$.

Preliminary performance tests of the APF linac structure were made in the following way. The APF section, which has no provisions for rf excitation, was bolted to another short linac section that does have such provisions. A 
special drift tube, containing an energy-discrimination foil (600 keV) followed by a Faraday cup, was installed between the structures to adjust the resonant frequency of the combination and to provide a minimal beam-diagnostics capability. The combined structure requires a total $\mathrm{rf}$ power of about $700 \mathrm{~kW}$ to reach the design excitation.

The combined structure was driven to a peak power of about $850 \mathrm{~kW}$, corresponding to an average axial electric field approximately $10 \%$ higher than the design value of $6 \mathrm{MV} / \mathrm{m}$. The maximum surface tields were in the vicinity of $28 \mathrm{MV} / \mathrm{m}$.

The 250-keV proton beam from the PIGMI injector was used for the preliminary beam tests. The net current signals from a Faraday cup at the entrance to the linac, the energy-discrimination foil in the special drift tube, and the Faraday cup in the special drift tube were monitored as a function of $r f$ power, injector voltage, solenoid current, steering current, buncher excitation, buncher phase, and ion-source parameters. Some of these data are presented in Fig. 33.

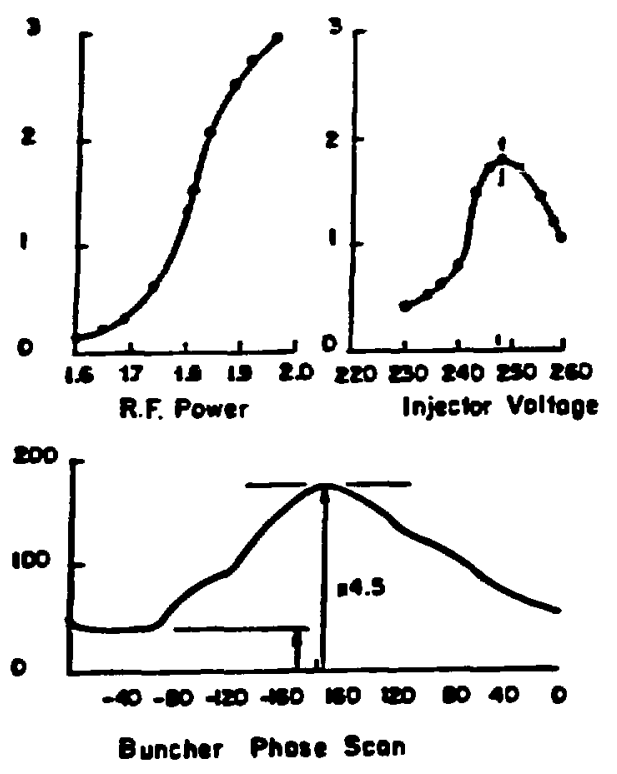

Fig. 33.

The APF performance data.

When protons with an energy $<600$ keV enter the special drift tube, they are absorbed in the foil and contribute to the signal from that element. Protons entering the special drift tube, with an energy $>600 \mathrm{keV}$, pass through the absorber and contribute to the signal from the Faraday cup within the drift tube. Stray electrons from the foil and Faraday cup are returned to their original element by a high-voltage supression ring. High-energy electrons originating in the accelerator are excluded by a l-kG magnet ic field produced by a pair of permanent magnets. The signal from the Faraday cup is taken as a measure of the accelerated beam current. 
Figure 33 shows the dependence of accelerated current, $I_{b}$, on rf power. The threshold for acceleration ( 1.75 in the arbitrary units given here) corresponds to an rf power of $750 \mathrm{~kW}$, and the peak acceptance corresponds to an rf power of $850 \mathrm{~kW}$. These data are in fair agreenent with our expectations.

At the optimum excitation, the percentage capture of a well-collimated beam is $15.7 \%$ or \pm 28 degrees of phase. This is in good agreenent with the expected performance.

The dependence of accelerated beam on injector voltage is shown in Fig. 33, revealing a rather sharp peak at 248V. The width of the peak agrees with the design calculations. The apparent deviation of the peak position from the design value of $250 \mathrm{kV}$ may be real or may be due to an error in the calibration of the voltage measurement. Figure 33 also shows the dependence of accelerated bean on buncher phase for the buncher excitation that produces the maximum accelerated beam at the optimum phase. The general features of this phase-scan look correct, but the detailed shape has not been analyzed.

The maximum current accelerated to date is $3 \mathrm{~mA}$. There are a number of hardware probiens and it is hoped that this limit can be raised in the future.

VI. DTL SECTION

The PIGMI Quad Famp Tank was completed and delivered at the same time as the AFF Tank. Fabrication at the nonmagnet-loaded drift tubes (threestermed) was completed, except for copperplating. Fabrication of the magnetloaded drift tubes was completed and they are now ready to load in the magnets. All drift-tube stem hardware was completed.

The PIGMI Phase Ramp and DTL tanks were completed and delivered in December 1979. The tuning-slug/vacuum port for the Phase Ramp tank was modified to allow placement of the ion pump on top of the tank. All nonmagnet-loaded (single-stenmed) drift tubes for the Phase Ramp and DTL tanks were completed, except for final stem machining and copperplating. All magnet-loaded drift tubes were completed to the point of being ready to receive the magnets. All drift-tube stem-termination hardware was completed.

The PIGMI DTL tank is being assembled. The DTL tank has been mounted on an alignment stand and the downstream tankhead installed. The tank will 
contain one nonmagnet-loaded drift tube and two magnet-loaded drift tubes. The nonmagnet-loaded drift tube is complete except for copperplating, and is installed temporarily in the DTL tank. The magnet-loaded drift tubes are ready to receive the magnets, which also are ready. Loading and quadrupole field measurements will commence as soon as manpower is available, probably not before the summer of 1980 .

Alignment stands and other miscellaneous components, fabricated for the Quad Ramp, Phase Ramp, and DTL tanks, are being used for the RFQ POP tests.

\section{PERMANENT-MAGNET QUADRUPOLES}

The PIGMI Prototype design calls for 18 quadrupole-loaded drift tubes, each quadrupole having a unique length and strength. For fabrication purposes each quadrupole falls into one of six geometry groups. The gradient of each assembly is adjusted by grinding flats to an appropriate septh on each of the four circular pole pieces. The geometry of each assembly is common within a group, in the spacing of the pole pieces, and in the shape of the yoke.

One assembly from each quadrupole group has been fabricated and assembled. The pole pieces for each assembly have been ground, measured, and reground until the proper gradient was obtained. This process required the development of a grinding fixture that could hold a pole piece to reproduce the same orientation for the removal of a few mils of material. In addition, a bookkeeping system kept track of the numerous pieces during the grinding and measuring operations. After each grinding operation, the magnetic properties of the assembly were measured: the transverse gradient in four orientations, the effective length at four points, and the harmonic content of the field. Transverse fields and effective length were measured with a Hall-effect probe, the harmonic content of the field was measured, using a rotating coil and spectrum analyzer.

Following each grinding and measuring operation, the data were analyzed to predict how much more material should be removed to achieve the required properties. This process was completed for one quadrupole assembly in each geometry group. 


\section{DEVELOPMENT OF THE RADIO-FREQUENCY QUADRUPOLE STRUCTURE}

The second linear accelerator structure studied on the PIGMI test stand was the RFQ. Its function is to accept the $0.10-\mathrm{MeV}$ unbunched deuteron beam from the injector, to bunch, and to accelerate it to an energy of $0.640 \mathrm{MeV}$. The basic concepts of the RFQ are described under the FMIT program, Sec. I, I-B.

\section{BEAM DYNAMICS}

The particle-dynamics program, PARMTEQ, is a program similar to PAR.MILA, and is designed and written for studying RFQ linacs. Like PARMILA, PARMTEQ allows much flexibility, particularly in the specification of input-particle distributions and in output formats.

The RFQ Linac (or the Space-Uniform Focusing 'inac, as it is called by the Russians) has some unique properties that should allow a high-capture efficiency of low-velocity beams. The tranverse focusing strength is approximately independent of position along the structure, but varies sinusoidally in time. High focusing can be achieved and, because it is electrostatic, can be independent of particle velocity. At any given instant the beam would appear to have approximately the same elliptical cross section throughout the linac. The acceleration and the $r f$ defocusing forces both depend on position along the linac, as well as on time.

Because of these differing properties, one wonders whether to use time or distance along the linac as the independent variable. In a conventional linac, one would naturally use distance, and PARMILA is structured to do so. Being similar to PARMILA, PARMTEQ is also structured this way, and the coordinates of all the particles are calculated at discrete points along the linac. But to properly calculate the space-charge forces, one needs to know all the particle coordinates at a given instant.

Consequently, before the space-charge impulses are applied in PARMTEQ, the transverse coordinates of each particle are transformed to their values corresponding to a specific time, assuming that the particle feels only the local $r$ quadrupole field. After applying the space-charge impulse, the coordinates are transformed back to their values corresponding to a specific location along the linac.

For beam-dynamics purposes, each linac cell is divided into $\mathrm{N}$ segments, where $\mathrm{N}$ is typically 4 . The space-charge impulses are applied once per cell at the time when the electric field is zero, at which time the "matched" beam 
would have a nearly circular cross section. In computing the space-charge forces, the effects of an arbitrary number (typically 5) of adjacent bunches on each side of the particle bunch is included. This requires a recalcuation of the space-charge table every few cells, depending on how rapidly $B r$ is changing. The CDC 6600 computing time required for 500 particles, when each cell is divided into 4 segments when space charge is included, is about 1 minute for 10 cells.

\section{RFQ FABRICATION}

Copper tubing, 6.125-in. 0.d. by 0.192-in. wall, was chosen as the main structural element for the cavity. Square ends were brazed to the tube, then accurately machined and faced to length. The ends form three mutually perpendicular planes; two are parallel to, and equidistant from, the cavity center line. In this form, the part is easily indexed and clamped in jig boring and other machine tools, where dowel pin and screw holes can be accurately located and drilled.

The vane bases are machined from solid OFHC copper. Slots are ade to receive copper tubing for cooling water. The bases, including water tubes, are located in the cavity with dowels and screws and the whole assembly is then furnace brazed.

The RFQ is suspended inside another cylindrical rf cavity by means of two copper disks. Contact fingers connect the inner surface of the outer cavity through the disks to the outer surface of the RFQ, forming a coaxial line terminated at each end by an electrical short circuit. The outer cavity, or manifold tank, is connected to vacuum and cooling-water services.

The rf power is fed through a wave guide to the manifold tank. The magnetic field couples through the angled slots in the RFQ side walls to magnetic fields inside the RFQ. Power is uniformly distributed to all four chambers of the RFQ.

Each end of the cavity is closed by a disk assembly containing four smarl tuning slugs, as shown in Fig. 34 . The slugs vary the capacitance between the vane ends and the cavity and they are used to adjust the electrical field distribution along the axis of the RFQ. 


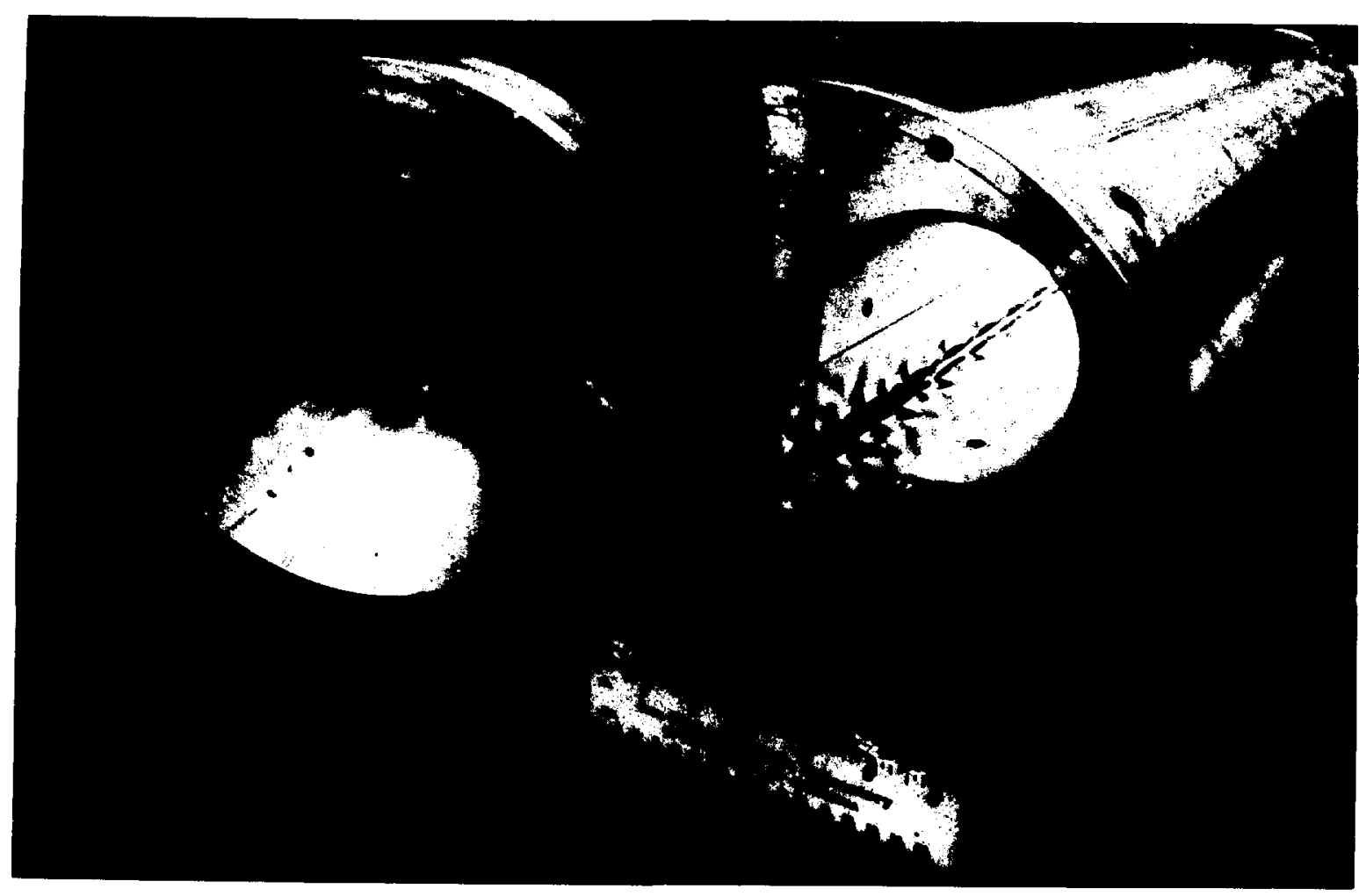

Fig. 34 .

Low-power RFQ test cavity.

Fabrication of the vanes incorporates a three-axis, numerically controlled, vertical mill. Normally, this machine reads its control data from a punched paper tape that has a capacity of about 800 data blocks. The vanes require approximately 20000 data blocks for an adequate description.

A spherical tool is used to cut the vanes. The path that the tool center follows is in the $x y-p l a n e$ and must consist only of straight lines and circular arcs. As each cross section is cut, the machine table moves an increment $W_{z}$ along the vane's longitudinal axis. The process is slow ( $W z=0.020$ in.), moving 1-2 inches per hour in the z-direction.

If the radius of the tool were zero, the tool path would be identical to the cross section of the vane. However, with a finite tool radius, the cutting path of the tool is not always in the same plane that the tool travels. The deviation depends on both the cutter radius and the slope of the vane. The program that computes the machine instructions compensates for this error. 
Development of the disk-and-washer linac structure for the PIGMI applications has continued throughout the present reporting period. The structure is a standing-wave linac and offers efficient acceleration at proton energies > $150 \mathrm{MeV}$. The structure's field distribution is extremely stable as a result of the strong intercell coupling and the $\pi / 2$ nature of the operating mode.

The once-favored radial-washer supports have proven to be too lossy for further consideration. Current efforts are directed toward the development of T-shaped washer supports because of their minimal perturbation of the cavity fields and their fore/aft symmetry. Techniques are being developed to counteract the perturbations that they introduce into the structure and coupling.

The geometry of the DAW linac structure (without washer supports) has been optimized with the aid of the rf cavity calculational program, SUPERFISH. The half-cell geometry, shown in Fig. 35, is characterized by the 10 quantities $L, G, R_{C}, R_{D}, T_{D}, R_{W}, T_{W}, R_{H}, R_{N}$, and $\theta$. A list of these quantities, optimized for the PIGMI application at $1320 \mathrm{MHz}$ are given in Table $\mathrm{V}$ for a range of beta from 0.4 to 1.0 in steps of 0.05 . For this application, a number of quantities were held constant, independent of beta; namely, $R_{C}$, $T_{W}, R_{H}, R_{N}$, and $\theta$. Figure 35 shows typical sections of this structure for beta equal to 0.5 and 0.8 .

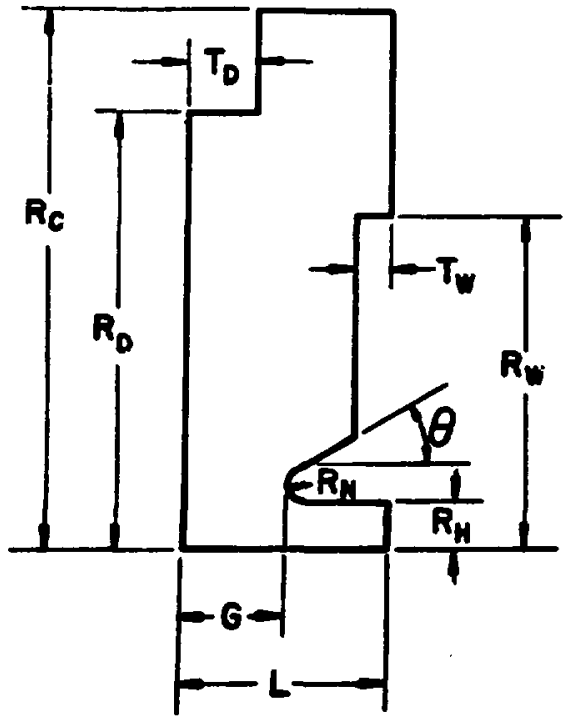

Fig. 35.

The washers are supported in pairs from a number of T-shaped supports. The radial portion of these supports lies along an equipotential of the cavity fields, and the longitudinal portion of these supports lies in regions of low electric field. Consequently, this support geometry represents a minimal perturbation to the frequency of the accelerating mode.

On the other hand, these supports do represent a significant perturbation to the frequency of the coupling mode and to the cell-to-cell coupling 
TABLE $V$

PARAMETERS FOR THE PIGMI DAW LINAC STRUCTURE AS A FUNCTION OF BETA

(FREQUENCY $=1320 \mathrm{MHz}$, WAVELENGTH $=22.712 \mathrm{~cm}$ )

$$
R_{C}=16.600, T_{W}=0.400, R_{H}=1.111, R_{N} 0.250,=30^{\circ}
$$

\begin{tabular}{|c|c|c|c|c|c|c|c|}
\hline Beta & $L$ & $\mathrm{G} / \mathrm{L}$ & G & $R_{D}$ & $\mathrm{~T}_{\mathrm{D}}$ & $\mathbf{R}_{W}$ & $Z T^{2}$ \\
\hline 0.4 & 2.271 & 0.306 & 0.695 & 11.71 & 0.36 & 11.27 & 32.25 \\
\hline 0.5 & 2.839 & 0.363 & 1.031 & 12.06 & 0.61 & 10.95 & 45.37 \\
\hline 0.6 & 3.407 & 0.415 & 1.414 & 12.45 & 0.92 & 10.63 & 56.87 \\
\hline 0.7 & 3.975 & 0.462 & 1.836 & 12.87 & 1.30 & 10.31 & 66.42 \\
\hline 0.8 & 4.542 & 0.504 & 2.289 & 13.32 & 1.72 & 9.94 & 75.17 \\
\hline 0.9 & 5.110 & 0.545 & 2.785 & 13.86 & 2.18 & 9.52 & 82.80 \\
\hline 1.0 & 5.678 & 0.582 & 3.304 & 14.54 & 2.71 & 9.94 & 88.03 \\
\hline
\end{tabular}

constants. Left uncompensated, these perturbations would open a stopband in the mode spectrum, jeopardizing the structure stability, and would create
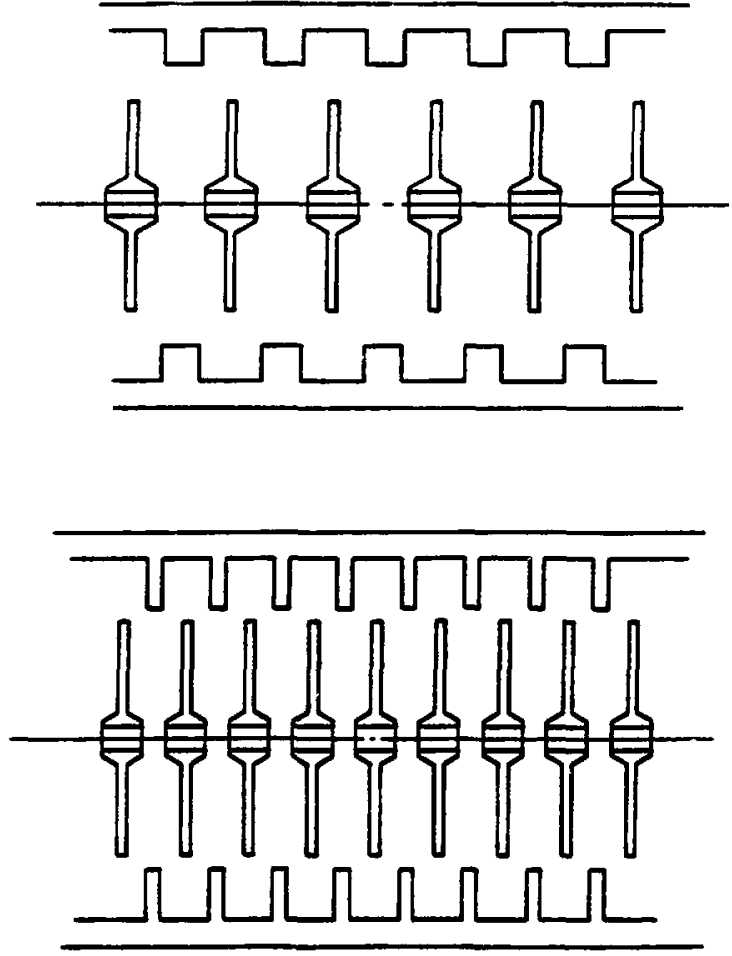

Fig. 36 .

Typical DAW sections for $B=0.5$ and $B=0.8$. a bilevel distribution in the excitation of the accelerating cells. Fortunately, it is possible to compensate these perturbations by minor changes in the geometry so as to retain a closed stopband, with its implied stability, and a uniform distribution of accelerating fields.

The magnitude of these perturbations, and the changes required to compensate for them, cannot be determined solely from SUPERFISH results, but depend in part on information gained from model cavity measurements. One approach is to measure the magnitude of the perturbations on the model cavity, and to learn from SUPERFISH the nature of the geometrical changes that are required to compensate for the effects 
of the perturbations. Armed with this information, it is a straightforward process to alter the cavity geometry so as to converge on the desired results.

Figure 37 shows some of the model's cavity hardware fabricated during this reporting period. Additional hardware is being fabricated from aluminum for $B=0.5,0.6,0.7$, and 0.8 . For fabrication and handling convenience, this hardware is being scaled down from $1320 \mathrm{MHz}$ to $2380 \mathrm{MHz}$.

XII. BRIDGE COUPLERS FOR THE DAW LINAC STRUCTURE

In long linac structures such as PIGMI, there is the need to break the structure into shorter sections to allow for the introduction of auxiliary apparatus such as beam-focusing quadrupoles, beam-diagnostic equipment, vacuumisolation valves, etc. In many cases, it is desirable to couple these sections into larger resonant units to reduce the required number of $r f$ power drive points and to lock the relative phase and amplitude of the fields in adjacent sections. Such couplers in LAMPF have been called bridge couplers, because

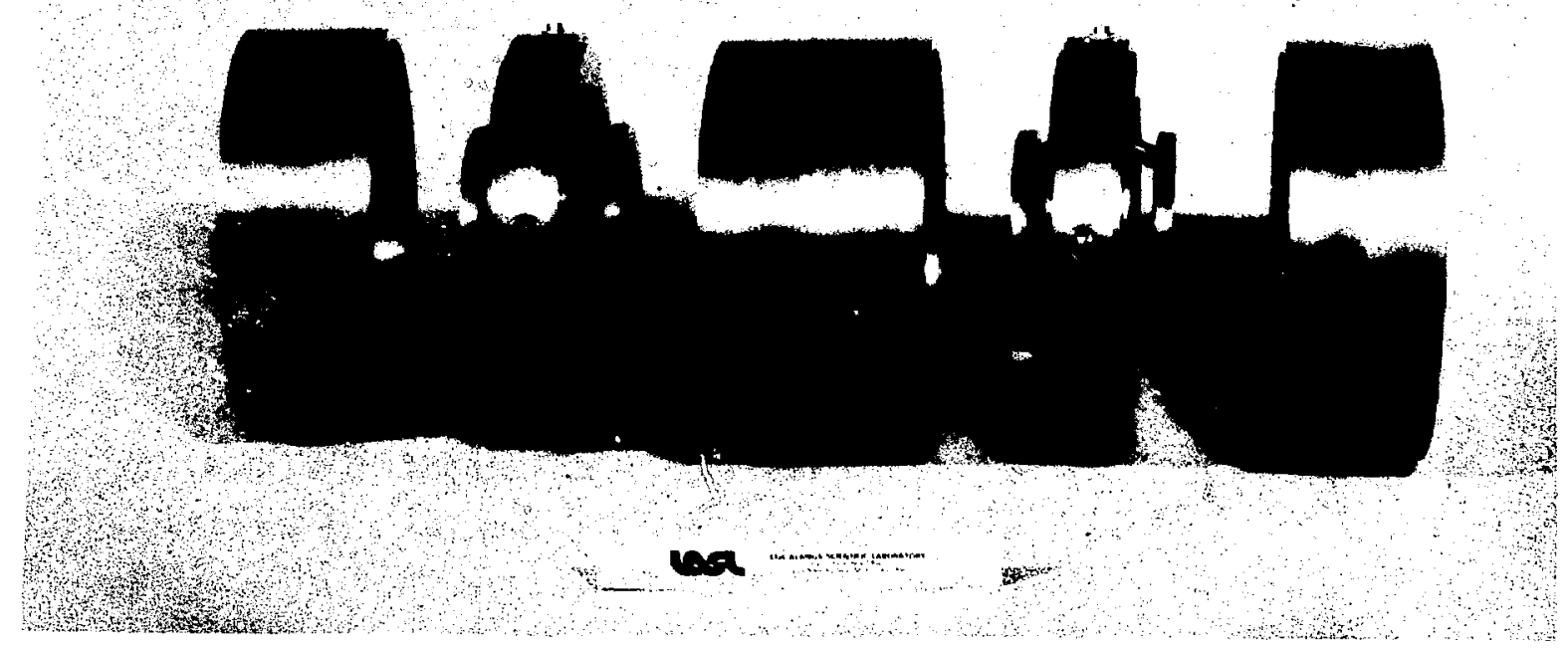

Fig. 37. DAW model cavity. 
they bridge the resonant properties of the linac structure around the auxiliary apparatus.

The DAW linac structure is a resonantly coupled ( $\pi / 2$ mode) linac structure with very large coupling constants. To take optimum advantage of the superior properties of this structure, any required bridge couplers also should be of the resonantly coupled type with large coupling constants. For practical structure tuning, the coupling of the bridge coupler to the linac structure snould represent a minimum distortion of the field patterns in either element.

It was recognized several years ago that the field patterns in the coaxial TEly mode were very similar to the field patterns in the plane of the washer of the DAW structure. A family of coaxial bridge couplers based on this geometry were proposed by J. J. Manca and E. A Knapp. The couplers in this family do not, in general, satisfy all the requirements of resonant coupling, which requires that resonance be maintained for both the accelerating moge and the coupling mode of the structure.

For accelerator applications, resonantly coupled bridge couplers must satisfy one beam-aynamics constraint and two rf constraints; the former requires that the length of the bridge coupler, as defined in Fig. 38 , be equal to $N_{B \lambda} / 2$, where $N$ is an interger; the latter requires proper accommodation for both degenerate modes of the linac structure. Until recently, we did not know how to satisfy both of these constraints simultaneously for an arbitrary particle velocity $B$.

For a simple cylindrical

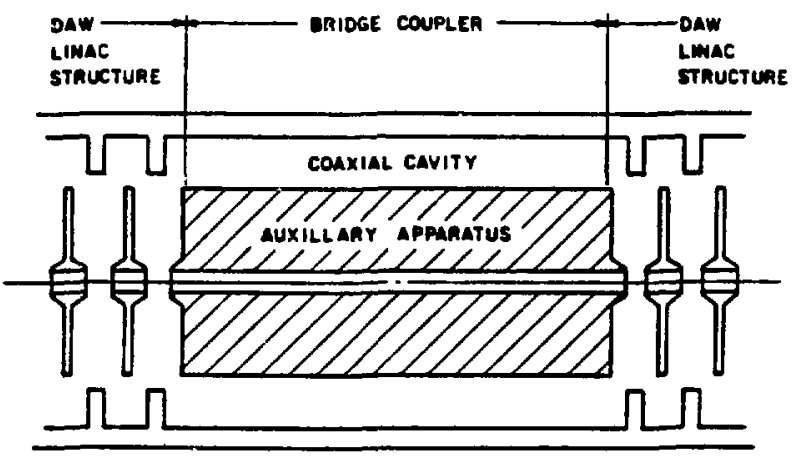

Fig. 38.

Resonantly coupled coaxial bridge coupler. coaxial cavity, shown in Fig. 38, the $r f$ constraints require that the bridge-coupler length be $M_{\lambda} / 2$, where $M$ is an integer. Furthermore, the beam dynamics requires that $M$ be of the same parity as $N$; that is, if $N$ is odd, $M$ must be odd, and if $N$ is even, $M$ must be even. This constraint on bridgecoupler length is incompatible with the beam-dynamics constraint on 
bridge-coupler length except for a few discrete values of $B$. Therefore, the simple cylindrical coaxial cavity is not a suitable solution for the general resonantly coupled bridge coupler.

One alternative is to choose the bridge-coupler length so as to satisfy the beam-dynamics constraint exactly, and to tune the resulting coaxial cavity so as to satisfy both rf constraints simultaneously. Two recently conceived schemes achieve these constraints.

The first scheme is based on our recent observation that there is a class of tuning perturbations that affects both modes in exactly the same manner. This class of perturbations guarantees that when one of the $r f$ constraints is satisfied, the other also will be satisfied.

The second scheme is based on the use of two different perturbations that affect the two modes differently, and the adjustment of these perturbations, in concert, until both rf constraints are satisfied. This scheme, although slightly more complex, may yield more appropriate geometries.

In either case, we can treat the bridge coupler as $M$ identical sections, where $M$ is chosen to be close to $B N$ and of the same parity as $N$. Thus, the basic section length is $(B N / M) \lambda / 2$. If $M$ is greater than $B N$, perturbations are required that lower the frequency of the two modes, and if $M$ is less than $B N$, perturbations are required that raise the frequency of the two modes. In practice, it may be desirable to constrain $M$ to be either greater than $B N$ or less than $B N$, so that only one type of perturbation is required.

The class of perturbations, having identical effects of both modes, is limited to symetrical perturbations centered on the $1 / 4$ and $3 / 4$ locations within the basic bridge-coupler section. This implies a fundamental periodicity of the bridge-coupler geometry of one-half the basic section length.

Figure 9 shows a bridge coupler of the type for $N=7, B=0.6$, and

$M=5$. The power dissipation of the accelerating mode in this bridge coupler is 1.15 times the dissipation in the accelerating cavity.

Figure 10 shows a bridge coupler of the second type for the same values of $N, B$, and $M$, where two different perturbations are used and adjusted, to satisfy the two $r f$ constraints simultaneousiy. The power dissipation of the accelerating mode in this bridge coupler is 1.21 times the dissipation in the accelerating cavity. 


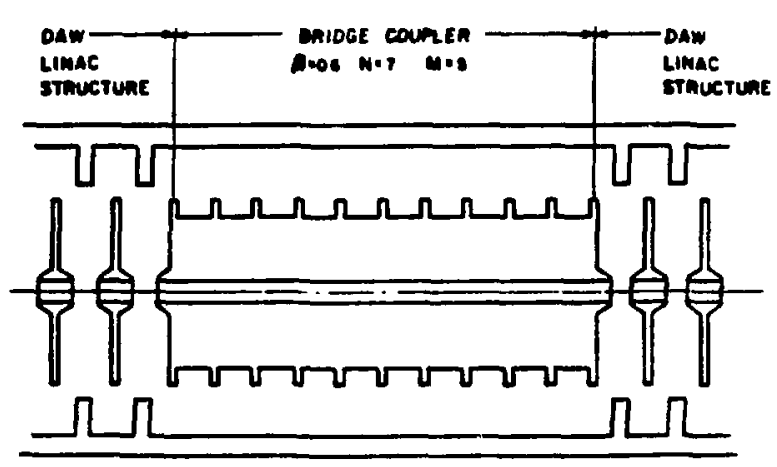

Fig. 39.

Type 1 bridge coupler.

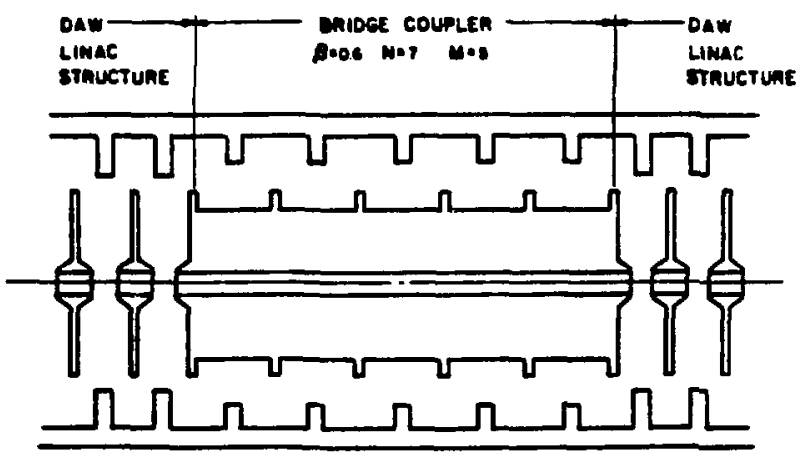

Fig. 40.

Type 2 bridge coupler.

For the PIGMI application, it appears that single-cell bridge couplers $(N=1)$ may be adequate to house the required apparatus within the linac structure. Figure 41 shows the $N=1, M=1$ bridge couplers of the second type for the values of $B$ equal to 0.5 , $0.6,0.7$, and 0.8. The power dissipations of the accelerating mode in these bridge couplers are $0.60,0.64,0.67$, and 0.68 times the dissipations in their respective accelerating cavities.

Figure 42 shows the field distribution for the accelerating mode and the coupling mode for the some four values of $B$. The resonant frequency in each case is $1320 \mathrm{MHz}$.

If the power dissipation in the bridge couplers is considered excessive, it should be possible to modify the geometry at each end of the bridge coupler so as to reduce the excitation of the bridge coupler without affecting the excitation of the accelerating structures. It should also be possible to implement a movable perturbation that would affect the relative excitations of the adjacent accelerating structures.

In the center of each of the $M$ identical bridge coupler sections is a region of high magnetic field and zero electric field. The effect of a radial short in this plane on the acceleraring and coupling modes is equivalent to the effect of the tank and terminations an the two modes. Conducting radial supports in this plane have a negligible effect on the accelerating mode and a tolerable effect on the coupling mode. 

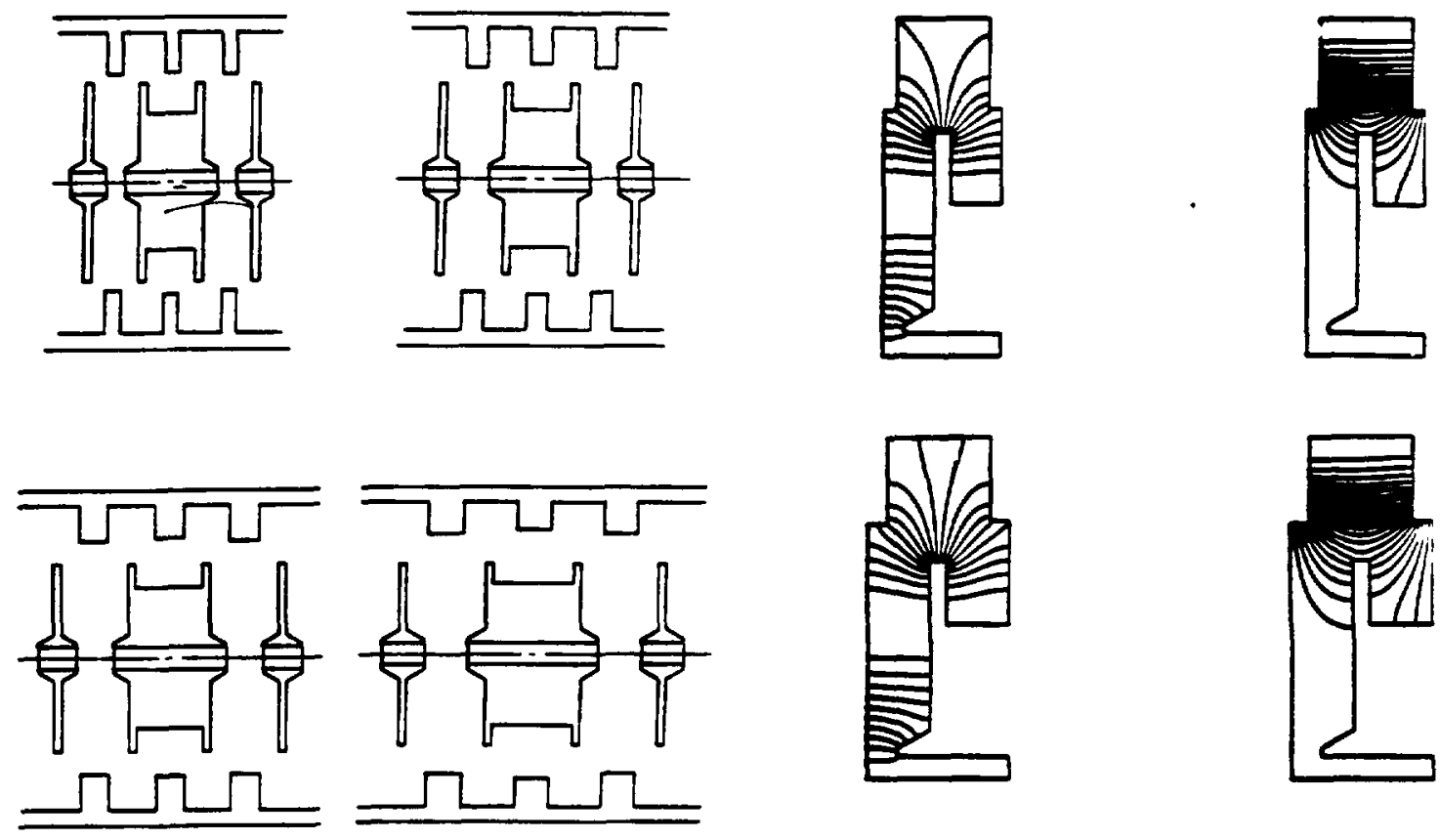

Fig. 41.

Single-cell bridge-coupler geometry for $\beta=0.5,0.7$, and 0.8 .

Hollow conducting radial supports provide a suitable channel for the services required by the auxiliary apparatus housed inside the bridge coupler, such as cooling water for the bridge parts, power leads for electromagnets, signal leads for beam diagnostic devices, and control rods for mechanical devices. There may be some advantage in selecting $M$ (and hence $N$ ) to be odd. With this constraint, midplane of the bridge coupler is a suitable plane for the support structure.
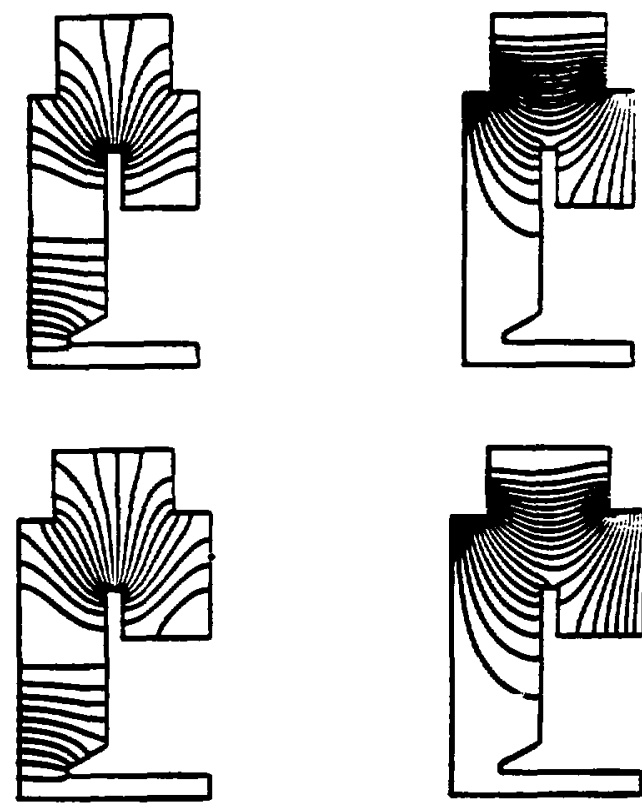

Fig. 42. Single-cell bridge coupler field distribution for $\beta=0.5,0.6,0.7$, and $\mathbf{0 . 8}$.

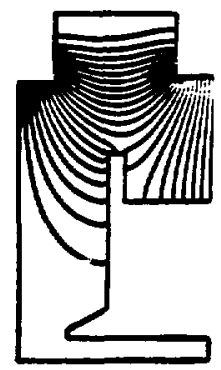


XIII. FIELD GRADIENT AND AVERAGE POWER ACCELERATOR STRUCTURE TEST

Essential to the PIGMI concept is the demonstration of the viability of the selected design acceleration gradients, and the accelerator structure compatibility with the related average power. Both of these parameters have been affirmed, using structures typical of those required for the low-frequency, low-beta portion of the PIGMI accelerator. These tests included both the Alvarez and RFQ structures. Similar testing of the DAW structure proposed for use in the higher-frequency, higher-beta portion remains. The 22-cell, $B=0.7$, DAW structure, as shown in Fig. 43 , will be used for this test. The selected PIGMI design gradient $(9.0 \mathrm{MV} / \mathrm{m})$ implies a maximum surface gradient somewhat in excess (15\%) of the Kilpatrick Criterion, a semiempirical guide to sparking threshold. For the selected test structure, a peak rf power of $1.4 \mathrm{MW}$ at an average $r f$ power of $5 \mathrm{~kW}$, represents the minimum requirements. Ideally, one would push the structure to sparking, with the $r f$ power requirement increasing with the square of the gradient.

Two high-power, pulsed, klystron tube types are available for application to these tests. Both of the available klystron types satisfy the peakand average-power requirements of the planned tests. However, they differ significantly in their modulator requirements. One type, the $L-3707$, uses a nonintercepting, modulating anode. As such, the tube operates with a dc potential applied across the tube, which in turn is gated on and off by an essentially zero-power pulse applied to the modulating anode. For experimental application, this type of modulation offers maximum operating flexibility. The pulse length and pulse-repetition rate can readily be varied. The effective tube perveance and the tube operating voltage can be varied, so as to

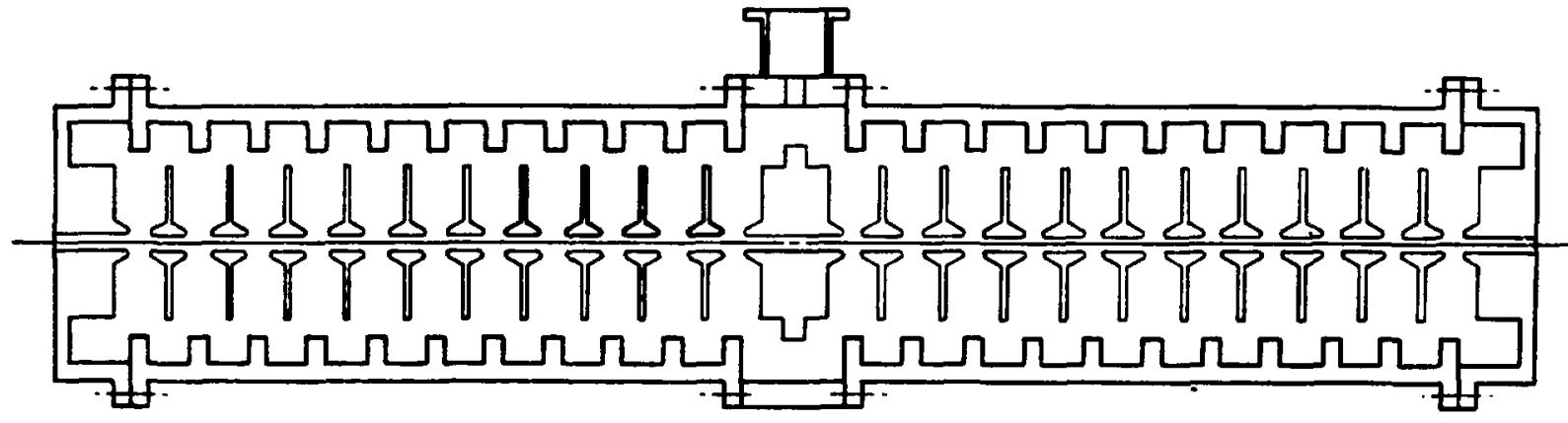

Fig. 43.

DAW power test assembly geometry. 
readily and efficiently tailor the power output to any changes in the experimental requirements. Additionally, the technology for this modulator type is well developed and was employed in the aforementioned low-frequency, low-betastructure tests.

The second klystron tube type available for use is the L-3661, which requires cathode modulation; that is, the full tube voltage and current must be gated. As such, the modulator must switch megawatts, as opposed to the essentially zero power required when using a modulating anode. On the other hand, the required dc voltages differ significantly with the cathode type, representing the more conservative stance. Conventionally, the cathode modulation is provided by a resonantly charged pulse-forming neiwork driving a step-up pulse transformer. The pulse length and pulse repetition rate are relatively fixed. Potentially more serious, this type of modulator is more difficult to engineer because of the long pulse $(-60 \mu s)$ requirement. The cathode-pulsed tube type is the more attractive choice for PIGMI application because commercially available cathode-pulsed klystrons offer significantly higher unit peak-power output. This in turn offers lower construction cost and increased operating reliability.

Becalise the cathode-pulsed tube was the preferred choice for an operational PIGMI facility, we decided to pursue the development of the required technology for this approach. While satisfying the basic requirements of the experiment, it will also provide an opportunity to develop the hardware and software unique to this approach and required for an operational facility. A resonantly charged pulse-forming network and pulse-transformer modulator has been designed. In combination with the L-3661 klystron, the program can test the 22-cell structure at gradients of up to 2.5 times the Kilpatrick criterion and at average powers of up to four times that projected by the design. The equipment required for this test will be assembled in space recently made available to the program by the Laboratory. The L-3707 klystron, the combination with the previously used PIGMI modulator, will be used as a contingency backup.

\section{PION-CHANNEL DESIGN}

For the treatment of tumors with pi-minus mesons (pions), a pion channel with a large solid angle is essential. A parallel beam of uniform density and reasonable size at the patient position is favored, from the practical point 
of view. A new pion channel with sixty toroidal current sheets and some solenoids is shown to meet these requirements. It would also be useful for some physics experiments using low-energy pion and muon beams.

The pion channel used at LAMPF consists of two bending magnets and eight quadrupole lenses and has a solid angle of about $15 \mathrm{msr}$. Even with a proton beam of $500 \mu \mathrm{A}$, it takes a fairly long time to treat a patient (15-20 min). Therefore, it is of interest to consider pion channels with a larger solid angle.

So $f a r$, two kinds of pion channels with a solid angle of 0.5 to $1.0 \mathrm{sr}$ have been constructed for medical application. One is tine double orange-sector pion channel at Stanford and SIN. The other is one with a shaped solenoidal field developed at Dubna. The latter type has recently been considered at TRIUMPF. In these channels, pions from the target are refocused to a point in space. However, treatment experience at LASL suggests that a parallel beam of unifor density is to be preferred because of its ease of use. The channel with an orange sector can collect many pions. We propose and analyze a new pion channel design where the pions are collected with an orange-sector magnet, and then made into a parallel beam by running the pions outside a special solenoid field.

Figure 44 displays an overview of the practical situation in the treatment of a patient. The position of the patient can be adjusted, depending on where the tumor is in the body. For a tumor lying near the surface of the body, the patient can be placed nearer to the main solenoids, whereas for a deep-seated tumor, he should be placed a little downstream to make use of the more parallel beam.

After bombarding the target, the proton beam is guided outside. This makes it easier to reduce neutron and $y$-ray background at the patient position from beam dump, as compared with the situation at SIN, where the proton beam is stopped between target and patient.

The pion-production angle of $\theta_{\pi}=\left(85 \pm 15^{\circ}\right)$ is selected in the present channel. It is probably the best choice, considering the angular dependence of pion-production cross section and a tolerable contamination of muons and electrons. 


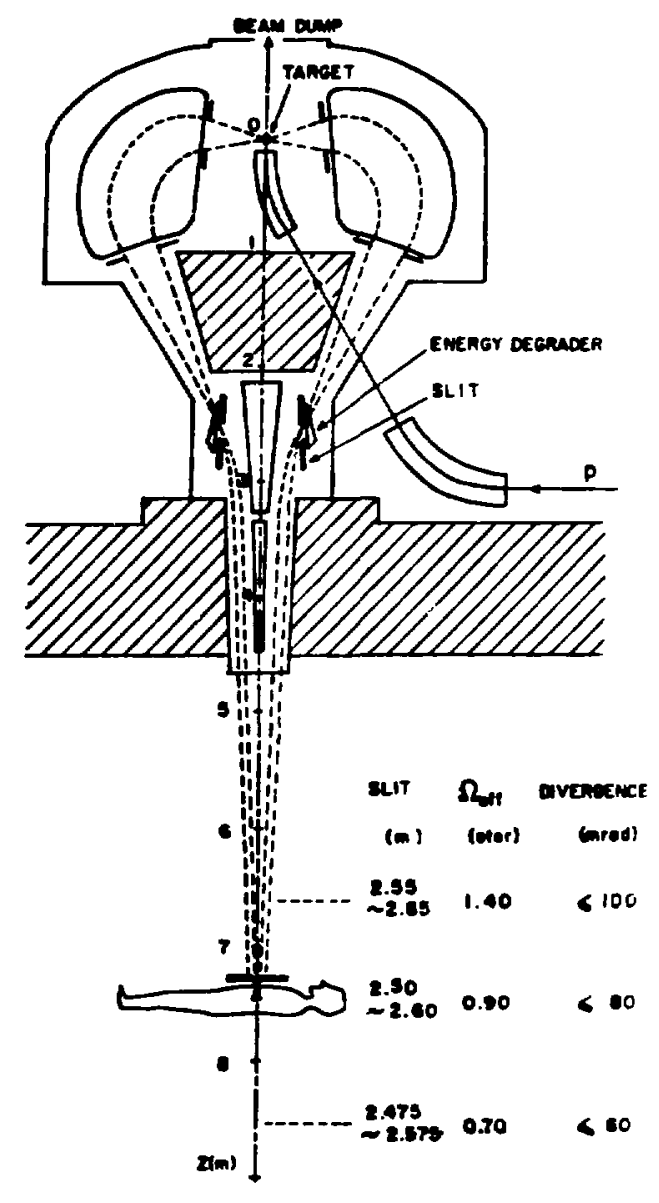

Fig. 44.

Practical situation of the present pion channel in the treatment of a patient.
The present channel would be useful for some physics experiments. In fact, if we do not mind the beam size of $r=20 \mathrm{~cm}$, we can attain a solid angle of more than $2 \mathrm{sr}$, while the resulting beam with a selected energy is fairly parallel.

In the present work, some basic characteristics of the current sheet and solenoid pion channel are explored in fair detail. However, prior to practical design considerations, additional work must be done. Some considerations are discussed below:

1) The bending magnets for the incident proton beam should be designed so that they do not interfere magnetically with the arrangement of the sector magnets and solenoids.

2) It should be established from what material each solenoid must be made. For the two main solenoids, superconducting coils

are appropriate, whereas the remaining solenoids can be made with conventional copper wire.

3) By tuning the shape of the current sheets, a sharper focus can be obtained. Refinement of solenoids would result in a larger effective solidangle.

4) It is estimated that an energy degrader can be used to reduce the energy width of particles at the patient position. The original particle distribution of the parallelogran can be converted into the rectangular form. Energy loss, straggling and variation in trajectory angle for each particle at the degrader will cause some variation from the rectangular form. The loss of 
beam intensity from multiple scattering in the degrader should be analyzed quantitatively.

5) It would be interesting to investigate the possibility of some kind of dynamic treatment. As the pion energy is increased, the layer where the pions are stopping goes deeper and deeper. The shape of each layer should be exactly matched to that of the tumor at that point. This can be done by changing the shape of the slit on the patient as we change the pion energy.

\section{ACHROMATIC BENDING SYSTEM}

A PIGMI accelerator would be $130 \mathrm{~m}$ long. One possibility of reducing the total length of the facility is to bend the linac back on itself at the halfway point. However, the $180^{\circ}$ bending system used for this purpose must have the very special properties of being both achromatic and isochronous to transport the proton beam without destroying the transverse or longitudinal dimensions of the bunch.

Two such systems have been studied. We expect the $180^{\circ}$ bending system to be positioned at about the halfway point in the linac structure corresponding to an energy of $250 \mathrm{MeV}$. The bending system would consist of several bending and quadrupole magnets. The problem is to find an appropriate combination of these magnets to satisfy both the achromatic and the isochronous conditions.

A flat, semicircular bending magnet $c$ an achieve iscchronous, but not acnromatic, bending. When two identical $90^{\circ}$ bending magnets with a quadrupole magnet located between them are used, a system $c$ an be made either achromatic or isochronous (depending on the field strength of the quadrupole magnet), but it cannot be mads simultaneously achromatic and isochronous. Therefore, three or more separate bending magnets are necessary to build the desired system. Two possible systems were studied.

Figure 45 shows an achromatic and isochronous system consisting of three bending-magnets. This system satisfies all of the necessary constraints. Particle trajectories of special interest are shown in Fig. 46. The z-axis coincides with the central ray, The $x$ - and $y$-axes indicate the deviation from the central ray in horizontal and vertical motions, respectively. A monochromatic beam, diverging at the entrance, forms an intermediate double-focusing image at the symmetry center of the second bending magnet $B_{B}$, and converges again to the exit. 
A representative trajectory of

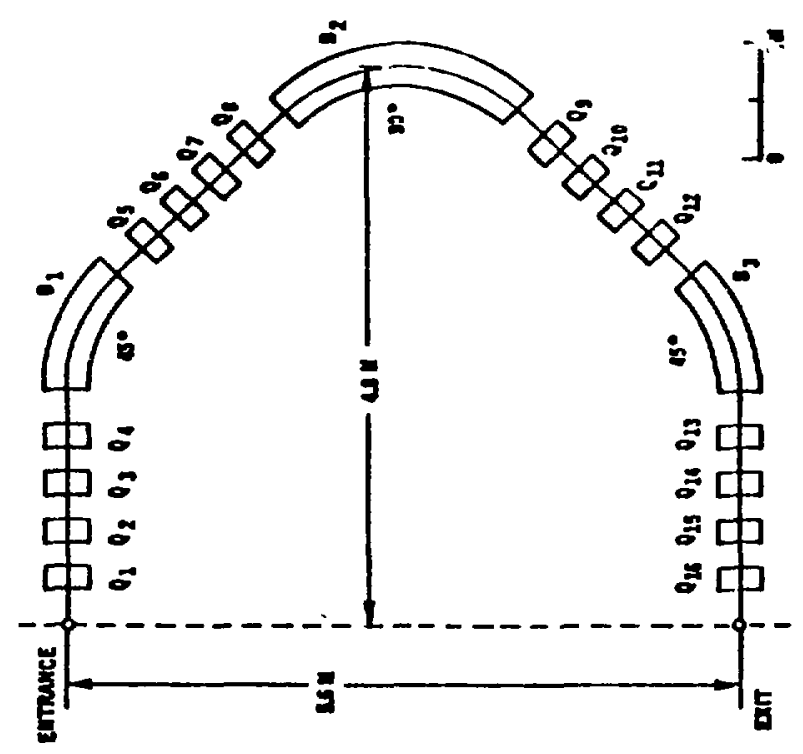

Fig. 45.

An achromatic and isochronous bending system -- System 1. (The bend radius of each magnet is $1.4 \mathrm{~m}$ ).

$\theta_{0}=4 \mathrm{mrad}$ and $\phi_{0}=4 \mathrm{mrad}$ is drawn with solid lines in Fig. 46. An incoming monochromatic beam, parallel to the central ray, again becomes parallel at the symmetry center $a^{-1}$ is transported to an outgoing $p$ 'el beam at the exit. A representative trajectory of $x_{0}=5 \mathrm{~mm}$ and $y_{0}=5 \mathrm{~mm}$ is drawn with dotted lines. An off-moment um particle, incoming on the central ray at the entrance, deviates horizontally from the central ray in the first bending magnet and travels on a dispersive trajectory, which becomes parallel to the central ray at the symmetry center, so the particle is again on the central ray at the exit. A typical trajectory for $\Delta p / p=0.2$ is shown as a solid line in the $x z-p l a n e$, labeled $\delta=0.2$. A relatively large dispersion at the symmetry center is necessary to make the system isochronous.

For this system, the separation between the incoming and outgoing beams is $5.6 \mathrm{~m}$, which makes it unattractive for some applications. To build a system with smaller separation, it is necessary to use more magnets involving bends to both directions.

A somewhat more complicated system, consisting of eight bending magnets, is shown in Fig. 47. Involving bends in both directions, this system is relatively large, but the separation between the incoming and the outgoing beams is only $2 \mathrm{~m}$. In the first half of the system, optical properties of point-to-point imaging and parallel-to-parallel transformation again are realized for the monochromatic beam. Therefore, any monochromatic beam can be transported to the one with the same phase space in the full system. off-momentum particles, incoming on the central trajectory, are dispersed horizontally by the first bending magnet $B_{1}$ to an intermediate image near the quadrupole $Q_{5}$. The angular dispersion at this point can yield a large 

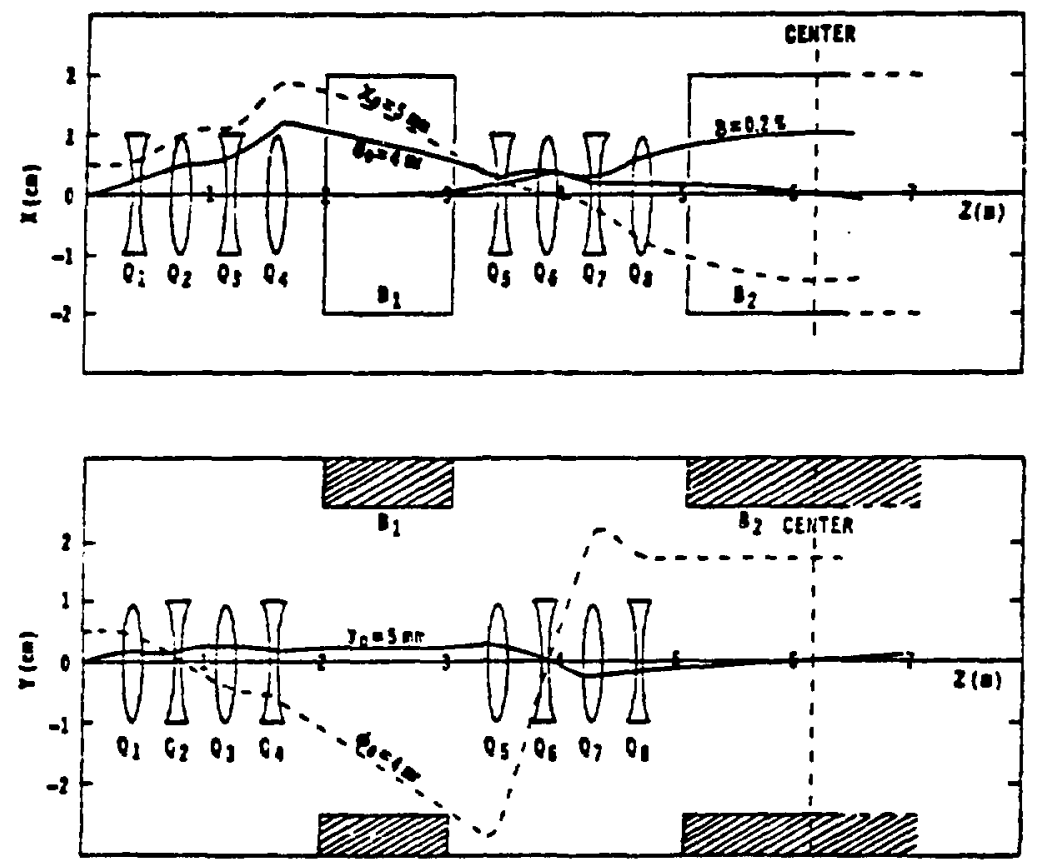

Fig. 46.

Particle trajectories of special interest in Sysiem 2. (The solid line and the dotted line show trajectories of particles of central momentum. The solid line labeled $\delta=0.2$ is that of an off-momentum particle.)

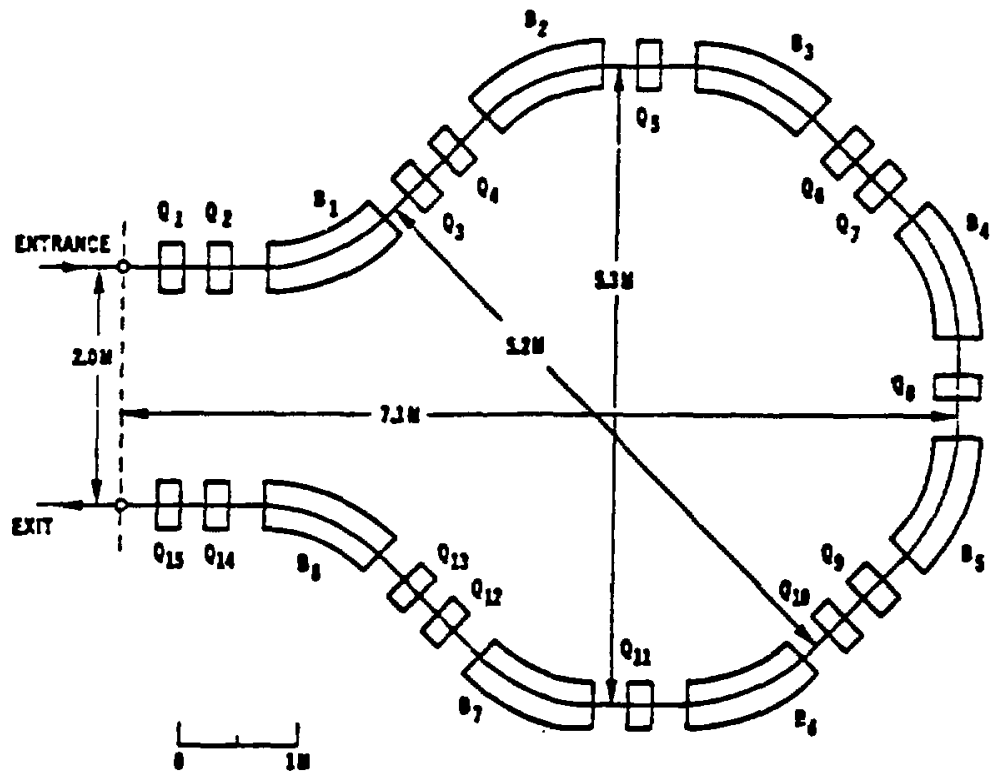

Fig. 47.

An achromatic and isochronous bending system that involves bends in both directions--System 2. (The bend angle of each magnet is $45^{\circ}$; the bend radius is $1.4 \mathrm{~m}$. All bending magnets are identical.) 
spatial dispersion at the symetry center and sufficient path-length difference to realize the desired isochronous condition in the full system.

The 2-m separation between the input and output beams will facilitate installation of both halves of the accelerator in the same beam channel, and this system can readily be designed for even smaller separations. Finally, it should be pointed out that all bending magnets are identical, which would be advantageous from the cost aspect. 


\section{SUMMARY}

The NBS-LASL racetrack microtron (RTM) is being built as part of an accelerator research project whose goal is to determine the feasibility of building a high-energy, high-current, continuous-beam electron accelerator, using beam recirculation and room-temperature $r f$ accelerating structures. This is in response to the Livingston Panel Report's recommendation that "accelerator research and development programs in room-temperature accelerator systems be supported." This is a joint project of the US Department of Commerce, National Bureau of Standards (NBS) and LASL. The work, started in October 1979, is supported by the Department of Energy, Division of Nuclear Physics.

The NBS-LASL RTM is being designed and built to develop the required technology for a large 1- to 2-GeV "National" accelerator and to prove experimentally that high currents $(550 \mu \mathrm{A})$ can be successfully accelerated. The advantages of an accelerator that employs recirculation and room-temperature rf are that, compared to a superconducting accelerator, a room-temperature machine is expected to have a much higher beam current capability; compared to a conventional pulsed linac, with a pulse-stretcher storage $r$ ing to achieve high duty factor, it should be considerably less expensive to build and operate and should have much better beam quaility.

The design parameters are chosen to be as applicable as possible to the larger machine. The RTM could serve as the injector prototype of a high-energy double-sided microtron, and the accelerating section for the RTM could serve as an accelerating module prototype of the high-energy microtron, which would require four such modules. The parameters chosen are the operating rf frequency $(2380 \mathrm{MHz})$, which is dictated by the commercial availability of a highpower $(450-\mathrm{kw}) \mathrm{cw}$ klystron and the desirability of subharmonic rf beam splitting to provide multiple, simultaneous, high-current beams to several users. A one-pass energy gain of $12 \mathrm{MeV}$ is essentially one-fourth the optimum energy gain, per pass, of the high-energy, double-sided microtron and only slightly less than the optimum energy gain for the NBS-LASL RTM. We chose 15

Æ Paper to be presented at the XIth International Conference on High Energy Accelerator, CERN, Geneva, July 7-11, 1980. 
circulations, which, with a 5-MeV injection energy, gives a 185-MeV final beam energy and a 550- $\mathrm{A}$ current. These parameters will be a good test for beam blowup problems. Under these conditions the beam loading in the main accelerating section will be about $33 \%$ of the input rf power, a value comparable to the larger machine's projected design.

The disk and washer accelerator structure being developed at LASL will be the accelerator section of the RTM. The rf drive systems and the rf beamdiagnostics systems will also be developed and built at LASL for the RTM. The beam-transport system will be designed and built at NBS. The RTM will be assembled in a well-shielded room in the NBS linac complex. A high-resolution electron spectrometer, in an adjacent room, will provide a unique capability for studying the beam properties of the RTM.

We are in the process of procuring the major components of the rf system and assembling a test section of the disk-and-washer structure.

\section{INTRODUCTION}

In principle, the electromagnetic interaction is the ideal experimental tool for studying nuclear structure because it is weak and well understood. In fact, the study of electronuclear and photonuclear reactions has contributed greatly to our understanding of nuclei. Nevertheless, electron accelerators are relatively little used compared to proton and heavy ion machines, largely because it is difficult to perform experiments with electrons or photons. These difficulties arise from the small size of electromagnetic cross sections, the unavailability of monoenergetic photon sources, and the poor duty cycle of most existing electron accelerators. Recent technological advances have raised the exciting possibility that new types of electron accelerators can be built, at acceptable cost, that will provide high-current, continuous-duty (that is, cw) electron beams, thus alleviating the experimental difficulties mentioned above.

The NBS-LASL racetrack microtron illustrated in Fig. 48 is part of an accelerator research project whose goal is to determine the feasibility of building a 1- to 2-GeV, 100 duty cycle electron accelerator that can generate at least $100 \mu \mathrm{A} .{ }^{60,61}$ It is quite clear that the least expensive accelerator design that $\mathrm{c}$ an achieve these parameters is the racetrack microtron, or similar device, in which the beam is recirculated through the same accelerating structure many times. 

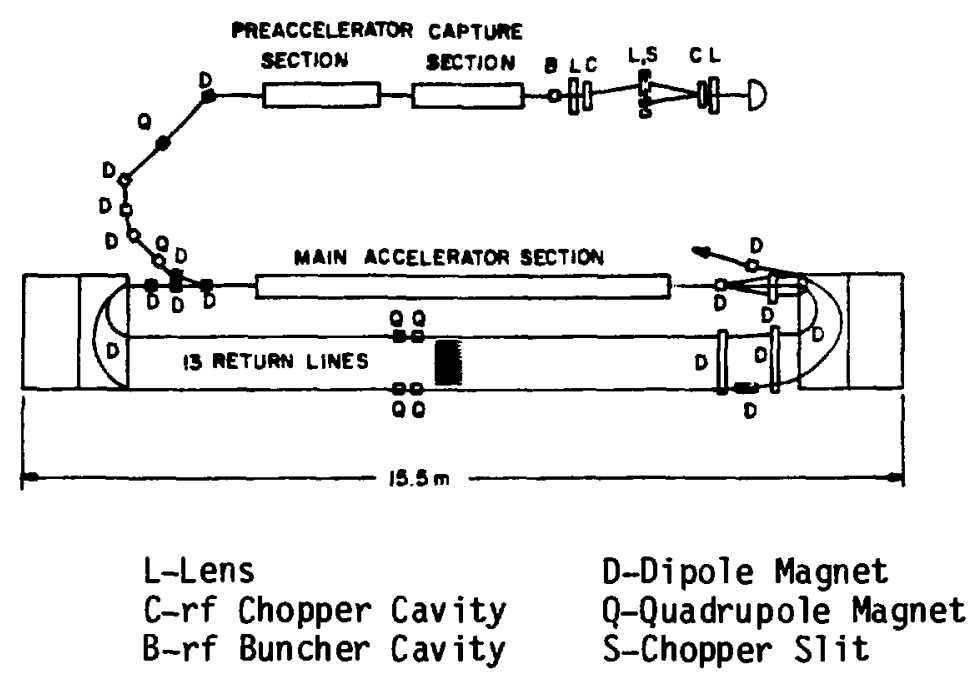

Fig. 48.

NBS-LASL Racetrack Microtron (15 passes, 12 MeV/pass, $185 \mathrm{MeV}$ ).

We designed the RTM to be as applicable as possible to the 1-to- $2 \mathrm{GeV}$ machine. The specific choice of the $2380 \mathrm{MHz}$ operating $\mathrm{rf}$ frequency was dictated by the desire to provide multiple, simultaneous, high-current beams to several users by subharmonic rf splitting and by the commercial availability of a high-power $(450 \mathrm{~kW}) \mathrm{cw}$ klystron. The $12 \mathrm{MeV}$ one-pass energy gain, which requires a magnetic field of about $1 \mathrm{~T}$ in the end magnets, is essentialiy one-fourth of the optimum energy gain per pass of a high-energy double-sided microtron and thus serves as a prototype accelerating module. The final $185 \mathrm{MeV}$ beam energy is estimated to be near the injection energy required by a high-energy double-sided microtron; thus the RTM serves as a prototype of the injector needed by the larger machine.

We have chosen room-temperature rf accelerating structure over superconducting structure for two reasons. First, a large amount of rf power is required to provide the beam power in a high-current accelerator; thus roomtemperature accelerating structures can have a high ( $r f$ to beam) power conversion efficiency. Second, the current in a microtron is expected to be limited by beam blomup caused by excitation of transverse rf modes by the beam. We expect the limiting current to be inversely proportional to the quality factor, $Q$, of the responsible $r f$ modes and inversely proportional to 
the number of passes. ${ }^{62,63}$ Because the Q-values are substantially smaller in a room-temperature $\mathrm{rf}$ accelerating structure than in a superconducting one, a much higher current limit is obtainable with a room-temperature structure.

\section{INJECTION SYSTEM}

The injection system must provide a $\mathrm{cw}$ electron beam to the microtron with the following specifications:

1. The injection energy must be $\sim 5 \mathrm{MeV}$.

2. The injected current must be continuously adjustable from zero to $0.6 \mathrm{~mA}$.

3. A pulsed beam mode must be available for tuneup and diagnostics with a 40 ns FWHM pulse length, which is shorter than the microtron's circulation time.

4. The normalized transverse emittance must be $<5 \pi \mathrm{mm} \cdot \mathrm{mrad}$.

5. The longitudinal emittance must be $<20 \pi \mathrm{keV}$ degrees.

The injection system will consist of a $100 \mathrm{kV}$.electron gun, an $\mathrm{rf}$ chopper-buncher system, a $2 \mathrm{MeV}$ rf capture section, an rf preaccelerator section for an additional $3 \mathrm{MeV}$ energy gain, and a beam-transport system to carry the $5 \mathrm{MeV}$ beam to the microtron. The electron gun will have a modulating anode for current control. The high voltage will be applied between the grounded final anode and the cathode, so that the control voltage applied to the modulating anode does not affect the beam energy. A pulser located in the high voltage terminal will provide the pulsing capabilities specified above.

The $r f$ chopper-buncher system consists of a pair of $r f$ chopper cavities, a phase-selecting aperture, magnetic focusing lenses, and an $r f$ buncher cavity. The chopped, bunched beam proceeds to the rf capture section where acceleration begins.

\section{MICROTRON DESIGN}

In an RTM, ${ }^{64}$ the beam is returned to the accelerating section by uniform field end magnets, as shown in Fig. 48. On successive passes, the beam must pass through the accelerating section at the same synchonous phase, $r$, of the $r f$ field. This resonance condition can be expressed by the relation $65^{r}$

$$
(2 \pi / c) \cdot \Delta V \cdot \cos \phi_{r}=v \lambda B
$$


where $\Delta V \cdot \cos \phi_{r}$ is the resonance energy gain per pass, $\lambda$ is the $r f$ free-space wavelength $(12.5963 \mathrm{~cm}), B$ is the end-magnet field strength, and $v$ the harmonic number. We have chosen to use $v=2$, which makes the spacing between successive return paths $d=v \lambda / \pi=8.019 \mathrm{~cm}$ (when $\beta=1$, the electron velocity in units of $c$ ). This spacing is sufficient to allow installation of independent steering and focusing elements on each return path. The major disadvantage of $v=2$ compared to $v=1$ is the reduced longitudinal phase acceptance of the RTM. Because of this, we will strive to keep the longitudinal emittance of the injected beam well within the design specification of $20 \pi \mathrm{keV}$ degrees. We will use a reverse return after the first pass through the main accelerating section. The second and subsequent passes will be in the opposite direction from the first pass. Sufficient focusing for the first two passes will be provided on the accelerator axis, whereas later passes will have focusing on the return paths. Extraction $c$ an be accomplished after any number of passes by a movable kicker magnet on the return paths. The kicker magnet deflects the beam on to a common extraction path from any return line.

The transport system needed to transfer the beam from the injector to the microtron must be a very high-quality achromatic system. The system chosen consists of two $90^{\circ}$ deflection achromatic subsystems in series so that the full $180^{\circ}$ system is also achromatic. The first $90^{\circ}$ subsystem has a quadrupole singlet at the ceitter and two dipole magnets, each providing a $45^{\circ}$ deflection of the beam. The second subsystem is similar to the first except that each $45^{\circ}$ bend is accomplished by a pair of magnets, with one providing a $15^{\circ}$ deflection and the other providing a $30^{\circ}$ deflection. This modification is used because the final magnet that provides a $15^{\circ}$ deflection is also one of the three injection chicane magnets.

The diagnostic information needed to adjust the transport system will be obtained from instrumentation packages located on the axes of the injector and microtron linacs. These packages will contain phosphor view screens, wire scanners, and $r f$ cavities for measuring $x$ and $y$ transverse position, $r f$ phase, and beam current. Phosphor view screens and wire scanners will be placed on the return lines near each end of the microtron. The rf position, phase, and beam-current sensors placed on the axis of the microtron linac will use the pulsed beam for obtaining position, phase, and beam-current information for each pass. The beam pulse is shorter than the circumference of one pass 
through the microtron, thus the beam pulse produces a train of pulses in the rf sensors, each pulse corresponding to one pass through the microtron.

IV. DISK-AND-WASHER ACCELERATING STRUCTURE

The RTM contains three $r f$ accelerating sections: the capture section and preaccelerator sections of the injector linac, and the main accelerating section of the microtron. The $r f$ accelerator structure will be the disk-andwasher (DAW) linac structure with T-shaped washer supports that is being developed at the Los Alamos Scientific Laboratory for both electron and proton accelerator application. ${ }^{66}$ The DAW is a standing-wave structure that provides efficient acceleration of particles with $\beta>0.5$. The field distribution in the structure is extremely stable as a result of the large cell-to-cell coupling and the characteristic of the $\pi / 2$ operating mode. Figure 49 shows the DAW structure with the washers supported in pairs from four T-shaped supports. The radial portion of these supports lies along an equipotential of the accelerating mode, and the longitudinal portion of these supports lies in a region of low electric field. Consequently, this type of support minimizes the perturbation of the accelerating mode. However, these supports significantly perturb the coupling mode. Left uncompensated, these perturbations would open a stop-band in the mode spectrum and create a bilevel distribution in the excitation of the accelerating cells. Fortunately, by experimenting with the geometry of a low-power test section, a technique has been developed to counteract these perturbations. The geometry of the DAw (without washer

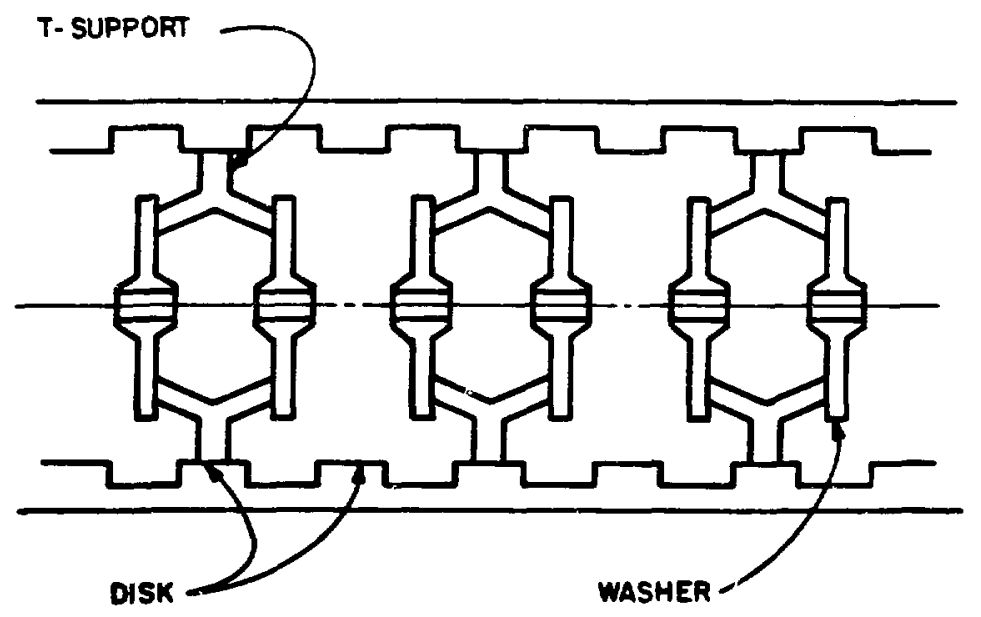

Fig. 49.

Disk and Washer Linac Structure. 
supports) was optimized with the aid of the computer program SUPERFISH. (This program can calculate the resonant electromagnetic modes in an rf cavity with cylindrical symmetry.) The perturbations caused by the supports are counteracted by a cut-and-fit procedure in which the disks, on which the T-shaped supports are mounted, are made thinner, and the radius of the outer wall of the cavities is made larger.

In addition to supporting the washers, the supports provide channels for carrying coolant to and from the washers. The practical limit for cooling the $2380-\mathrm{MHz}$ DAW structure is $\sim 25 \mathrm{~kW} / \mathrm{m}$. An effective shunt impedance of $\sim 100 \mathrm{M} \Omega / \mathrm{m}$ appears to be attainable in the $\beta=1$ structure and will result in an accelerating gradient >1.5 $\mathrm{MV} / \mathrm{m}$. Thus, the main accelerator section will be $\sim 8 \mathrm{~m}$ long to provide the $12 \mathrm{MeV}$ energy gain required by the design parameters. The injector linac will consist of $\sim 4 \mathrm{~m}$ of DAW structure. The first $2-\mathrm{m}$ section is a capture section that will increase the beam energy from $100 \mathrm{keV}$ to $2.0 \mathrm{MeV}$. In the capture section, the lengths of the $r f$ cavities increase smoothly to match the increasing electron velocity (from $B=0.55$ to $B=0.98$ ). The second $2-m$ section is the preaccelerator section that accelerates the beam to the microtron injection energy of $\sim 5 \mathrm{MeV}$. The construction of the $2-\mathrm{m}$ preaccelerator section will be undertaken as soon as the details of the washer supports and the compensation for the perturbations they produce is finalized. A low-power test section is presently being studied. The tests of the 2-m preaccelerator section will determine the cooling capacity and multipactoring properties of the DAW structure. 


\section{HEAVY-ION FUSION ACCELERATOR DEVELOPMENT}

\section{INTRODUCTION}

The possibility of using heavy-ion particle beams to drive inertially confined fusion targets has generated considerable interest in the accelerator community in recent years. This year's activities culminated in a workshop, of a series begun in 1976, held at Berkeley in November 1979. The AT-Division interest in heavy-ion fusion (HIF) centered on the possibilities of a revolutionary new type of accelerator structure, called the radio-frequency quadrupole (RFQ) structure for this application. The general properties of the RFQ, and other details of its development, are covered elsewhere in this report and in a number of papers cited as references.

\section{LINEAR ACCELERATOR CURRENT LIMITS}

For heavy ion fusion applications, there is a high priority in developing accelerating systems with the highest possible beam currents. In this context, we have investigated the origins of commonly used current-scaling laws, and have derived analytical expressions ${ }^{67}$ which apply to the RFQ, as well as to more conventional forms of linear accelerators. This activity has had two important results. First, we find that the current limit in a typical RFQ accelerator occurs at the end of the gentle buncher section. This result is in distinct contrast to a conventional linac with a bunched-beam input, where the current limit occurs at the linac entrance. The significance of this result is that, in a typical RFQ accelerator, the ions have gained a factor of ten in energy by the time they reach the end of the gentle buncher. Thus, in the RFQ, two desirable properties can co-exist. The input energy can be low, and at the same time the current linit can be a high value, characteristic of an energy about ten times the injection energy.

The second result comes from the use of two current-limit formulas: one derived from considerations of the longitudinal ion motion, and the other from the radial motion. If these two limits are equated, we obtain the highest current-carrying capacity and also we determine the corresponding value of the radial-focusing strength. In the RFQ, these considerations, together with a choice of a permissible value of peak surface rf electric field, determines the size of the RFQ aperture. More generally, for high-current linacs, these current-limit formulas help determine the values of ion energy which are 
appropriate to use for making transitions from one linac to another linac operating at a higher multiple frequency.

III. RFQ SYSTEM TO ACCELERATE $\mathrm{Xe}^{+1}$ IONS

It is generally acknowledged that one of the serious problem areas in accelerator systems for heavy ion fusion is the low-velocity accelerator section. Usually, beam-intensity limitations and significant radial emittance growth both occur in the first linac section, which follows the dc injector. We believe the RFQ to be a highly attractive possibility for minimizing these problems. It effectively focuses low-velocity ions, through use of the electric force, and it bunches an input dc beam in a highly efficient manner. It performs these functions with minimum radial-emittance growth and it accelerates the beam to an energy that is convenient for injection into a conventional linac structure. Also, as discussed above, the RFQ is consistent with the use of a relatively low-voltage dc injector.

To demonstrate this capability, we have designed an RFQ accelerator for $\mathrm{xe}^{+1}$ ions. ${ }^{68}$ In this example, a dc beam from a $0.24 \mathrm{MV}$ injector is bunched

TABLE VI

RFQ DESIGN FOR XENON

Input Current

Output Current

$30 \mathrm{~mA}$

Transmission

$29 \mathrm{~mA}$

96.9

NORMALIZED EMITTANCE (area/ $/ \pi)$

Input $\quad .100 \% \quad 0.0100 \mathrm{~cm} \cdot \mathrm{mr}$ 90\% $\quad 0.0073 \mathrm{~cm} \cdot \mathrm{mr}$ RMS $\quad 0.0017 \mathrm{~cm} \cdot \mathrm{mr}$

Ouput $\quad 90 \% \quad 0.0310 \mathrm{cmm}$ RMS $\quad 0.0068 \mathrm{~cm} \bullet \mathrm{mr}$ and accelerated to an energy of $5.0 \mathrm{MeV}$. We have used a frequency of $12.5 \mathrm{MHz}$ and a final synchronous phase of $-32^{\circ}$. The average aperture diameter is $3.6 \mathrm{~cm}$ and the overall length is $27 \mathrm{~m}$. Table VI gives the results of PARMTEQ beamdynamics simulation calculations for an input current of $30 \mathrm{~mA}$. The transmission efficiency is about $97 \%$ and the radial emittance growth for $90 \%$ of the output beam is a factor of 3.1. These results are very favorable when compared with other proposed low-velocity, heavy-ion accelerators. 
IV. MULTICHANNEL HEAVY-ION ACCELERATORS AND FUNNELING TECHNIQUES

Although we have demonsirated in the previous section that RFQ accelerators $c$ an be designed for high current, heavy-ion fusion applications require even higher beam currents. Usually, in the rf linac approach, it is proposed that these higher currents be obtained by combining the beams from a large number of individual linac channels. The technique used to successively combine the beams is called "funneling." 69 The basic element of this arrangement combines the beams from two separate linacs, operating with frequency $f$, into a single beam that has interlaced microstructure pulses. This combined beam $c$ an then be further accelerated in a single linac operating with frequency $2 f$. Successive applications of this technique allow the beams from $N$ initial RFQ structures of frequency $f$ and current $I$ to be combined into a single beam of current NI, that can then be accelerated in a linac operating with frequency $\mathrm{Nf}$. This procedure introduces no inherent radial-emittance growth from the funneling process, and should permit multiplication to the current values required from the linac portion of a heavy-ion-fusion driver. This funneling arrangement maximizes the efficiency of the overall system by having each longitudinal phase-stable bucket filled with beam particles. In addition, this technique maximizes the use of linacs operating with high frequency, and thus minimizes the size and power consumption of the overall system.

To facilitate the use of funneling techniques it is attractive to use a special form of the RFQ structure. For the low frequencies that are appropriate to the low velocities of the heavy ions from the dc injector, it is possible to use a compact array of four RFQ channels. ${ }^{69}$ The 4-channel module would be excited by radio-frequency power from an external resonant circuit. The adjacent channels of the array have small, transverse separations. This makes the beam paths required for funneling as short as possible, to minimize longitudinal debunching of the beam during the funneling operation. First, the four channels of the array would be combined in pairs to produce two beams. The beams to be combined would have their RFQ channels offset in the $z$ direction by their final value of $\beta \lambda / 2$. This offset allows use of a simple symmetric transport system to combine and interlace the microstructure pulses. 
Off sets would be applied throughout the whole system, so that after each funneling the puises would be properly interlaced. After the first funneling, the resulting pair of beams can be accelerated in a special form of Wideroe accelerator ${ }^{69}$ that has two beam channels. The beams would be focused by dc electric quadrupoles, and the two beam channels would be appropriately offset longitudinally to facilitate the next funneling operation.

As an example of the overall properties of such a system, a conceptual design of the linac portion of a $\mathrm{xe}^{+1}$ heavy-ion driver was devised. In this example, 32 RFQ linac channels (eight 4-channel modules) operating with a frequency of $12.5 \mathrm{MHz}$ were injected at an energy of $0.2 \mathrm{MeV}$. The $32 \mathrm{RFQ}$ beams were successively funneled and accelerated in Wideroe and drift-tube linacs and finally combined into a single $800-\mathrm{mA}$ beam that was then accelerated to $10 \mathrm{GeV}$. In this system, 84 of the total length was occupied by the two highest-frequency linacs that operated at 400 and $800 \mathrm{MHz}$. 


\section{GYROCON RF GENERATOR}

\section{SUMMARY}

The gyrocon is a high-power, high-efficiency narrow-band amplifier that operates by deflection modulation of an electron beam. The bunching is better than that in a klystron, especially for very high powers and UHF frequencies, so the overall efficiency and the maximum output power can be higher than in a klystron. The present theory includes the effects of large signals, space charge, and finite beam size. The equations of motion are relativistically correct, and the space-charge fields are correct to first order in v/c. The theory is derived and a computer code to solve these equations is discussed. The code is then used to obtain several specific examples of gyrocon designs that have significant advantages over klystrons or gridded tubes in the 0.2 to 3.0-GHz frequency range.

Several embodiments of the gyrocon are possible, and we discuss the radial style and the spherical style in this report. The radial style has a bender magnet to increase the deflection of the beam, whereas the spherical gyrocon does not employ the bender magnet. The optimum frequency range for the radial-style gyrocon is 0.2 to $1.0 \mathrm{GHz}$, and the optimium frequency range for the spherical gyrocon is from 1.5 to $2.5 \mathrm{GHz}$.

\section{INTRODUCTION}

The gyrocon amplifier, shown in Fig. 50, consists of an electron gun, a focus coil, a deflection cavity, a bender-and-focus system, an output resonant ring, and a collector. The electron gun produces a solid beam that is focused by the focus coil. The beam is then deflected by the magnetic field of a $\mathrm{TM}_{110}$ resonator that is excited by two inputs that are displaced $90^{\circ}$ in space and time. The resultant magnetic field rotates at the rf drive frequency exactly like the magnetic field in a two-phase motor. After traversing the deflection cavity, the envelope of the beam describes a cone, al though each electron has only an $r$ and $z$ component of velocity, and the beam would appear as an expanding helix if it could be photographed. The beam then travels through a bender region that increases the deflection angle of the beam by a static magnetic field. In the figure, the bender field is from a conical bender solenoid. Next, a pair of oppositely excited coils focuses the beam 


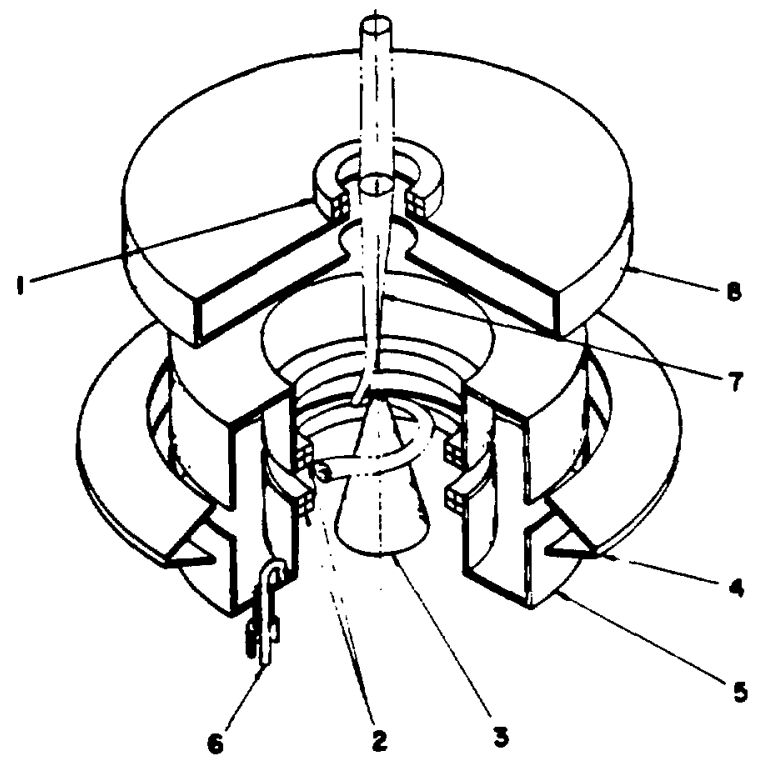

Fig. 50.

The radial-style gyrocon:

(1) the first-focus coil,

(2) the output-focus coils,

(3) the conical-bender solenoid,

(4) the collector,

(5) the output cavity,

(6) rf output line,

(7) the electron beam, and

(8) the deflection cavity.

toward the center of the output region, which is a section of coaxia) line operating at resonance in the $T E_{011}$ mode. The electrons give up their energy to the field as they cross the gap; if conditions are correct, over $90 \%$ of their $k$ inetic energy can be transferred to the field. The collector simply is the beam stop, but it is usually designed to absorb the full power of the beam. The beam is dece1erated by the radial component of the electric field in the resonator. The output fields are components of a fast wave, but because the azimuthal motion of the electrons is a deflection motion, the beam and the wave can be synchronous. The bunching in azimuth can be significantly better than the temporal bunching in a klystron, and we will show that operation is possible at very high beam voltages; thus large output powers are achievable.

Previous analytical studies ${ }^{70,71}$ of the gyrocon deflection-modulated rf amplifier have indicated that high dc-to-rf conversion efficiencies and acceptable gain and bandwidth for accelerator application can be achieved in the UHF to UHF frequency ranges. Two gyrocon devices have been built ${ }^{72,73}$ in the Soviet Union; the first one produces 1-MW CW at $181 \mathrm{MHz}$, whereas the second is a low duty-cycle amplifier that produces $40 \mathrm{MW}$ at $430 \mathrm{MHz}$. This second device drives the electron linac that feeds the VEPP $-4^{74}$ accelerator complex in Novosibirsk. The design theory ${ }^{73}$ for these gyrocons is now available in English. Recent analytical $75,76,77$ work at the LASL indicates that overall efficiencies over $80 \%$ can be realized from $100 \mathrm{MHz}$ to $3 \mathrm{GHz}$ with this type of amplifier. The efficiency increases as the output power is increased; 
thus the gyrocon is well suited for extremely highpower $r f$ systems. Output powers of more than 1-MW $\mathrm{CW}$ and pulse powers to $100 \mathrm{MW}$ appear feasible.

The radial-style gyrocon shown in Fig. 50 has been studied at LASL under the auspices of the Department of Energy and the Air Force Weapons Laboratory. The work has been progressing along two lines: the code development effort and an experimental effort. The experimental effort has been confined to studies of the radial-style gyrocon, but the codes are general enough to treat spherical output cavities and multiple deflection cavities, as shown in Fig. 51.

\section{THE MATHEMATICAL MODEL}

In the simplest terms, the gurocon is composed of several coils, two rf cavities, and an electron beam to interact with these fields and its own spacecharge fields. We analyze the gyrocon by integrating the trajectory equations through various regions of the gyrocon. A fixed system of cylindrical coordinates is used for the analysis, and the trajectory equations are integrated with time as the independent variable through the static and dynamic fields that describe the gyrocon. Space-charge fields are included by a line-charge

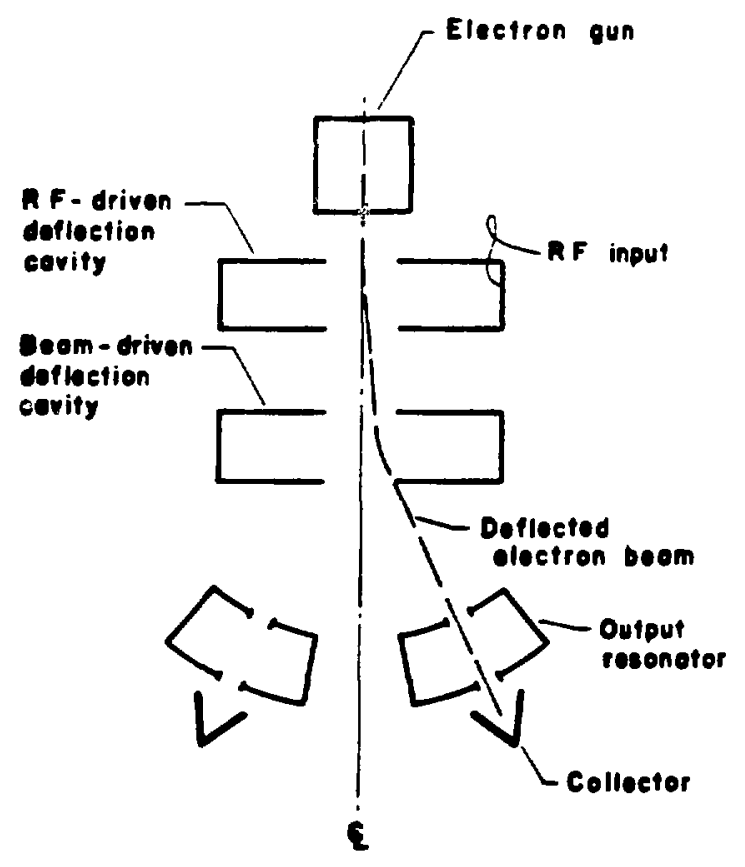

Fig. 51 .

The multiple-deflection cavity spherical gyrocon. model that has been modified to take into account the effects of the spiral nature of the beam and of relativity. The $r f$ fields are calculated for perfect, empty cavities. Thus it is assumed that the aperture and beam-loading effects on the cavities' fields are negligible.

The static magnetic fields of the various coils are calculated by subdividing each coil into one or more current loops, and summing the fields for all the loops. The equations describing the magnetic fields for a single, axially symmetric current loop are added to find the total static fields over a 
cylindrical magnetic grid, and the fields at each electron's position are linearly interpolated from tables. Fast elliptic integral routines are used in this computation. When permeable material is used to shape the magnetic fields, as in the gun region, the POISSON computer code is used to evaluate the magnetic fields and equivalent coils are determined from the calculated field on the axis.

The deflection cavity is cylindrical and can support $\mathrm{TM}_{110}$ modes. Two such modes, $90^{\circ}$ apart in space and time, can be combined to produce the traveling-wave fields. By integrating the tangential magnetic fields over the four surfaces of the cavity, the ohmic losses in the deflection cavity and the quality factor of the resonator are found. The effect of the apertures is neglected in the field and loss expressions. We also neglect any effect of the beam in exciting other modes.

The output resonator is considered to be a $\mathrm{TE}_{011}$ mode coaxial resonator. Three major assumptions are made about the fields: the effects of apertures are neglected, all higher order modes are neglected, and only the forward wave is considered in the interaction. Although the gyrocon can be used as a frequency multiplier, we consider only the amplifier case. This requires one azimuthal variation of the fields in the output guide. The circuit fields in the output cavity are expressed in terms of the cavity geometry and the arbitrary field amplitude $E_{0}$ and phase $\theta_{0} \cdot$ In practice, $E_{0}$ and $\theta_{0}$ are used as input parameters and are varied to maximize the overall efficiency. Once again, the tangential magnetic fields may be integrated over the cavity walls and the output power losses and $Q$ are found.

The space-charge fields are calculated with a rod model to minimize the computer time. At any instant of time the beam would appear as a conically expanding helix, although each electron's velocities are primarily in the $r$ and z-directions. We assume that electrons emitted at different times have identical trajactories, except for a change in angle by wst, where $\Delta t$ is the emission time difference. The space-charge field calculation is rather involved and is described in detail elsewhere. ${ }^{78}$ The procedure is described here briefly. The previous position of a reference electron is carried, and its old position is corrected by the wat correction. A vector between this old position and the present position is constructed and a plane perpendicular to this vector is calculated. The reference particle lies on this plane and all the other particles are projected onto the plane. Finally, each particle is 
assumed to represent a rod of charge and the electric fields between each rod are calculated. The magnetic fields are calculated from the electric fields and the field components are projected back to the particle's true position. The linear charge densities of the rods are corrected for the spiral motion of the beam and the slowing down of the whole beam.

The major assumptions in this model are that retardation and radiation modifications in the space-charge fields and all image charges are neglected. The model clearly breaks down when electrons are reflected or if the entire beam stops. The model has been thoroughly checked for nonrotating beams and the authors feel that the major effects of space charge are correctly accounted for.

A single cylindrical coordinate system is used in this analys is of the force equations. Because of the three-dimensional nature of the beam, the angular force equation must be integrated numerically. The energy equation is also integrated and used to test for numerical accuracy in the integration of the other equations. The total fields acting on each electron are found by adding the $r f$ space-charge fields and static magnetic fields to any $r f$ fields that are present in the various regions of the gyrocon.

\section{CALCULATION OF RADIAL-STYLE GYROCON PERFORMANCE}

A. The Calculation Method

The performance of the gyrocon is calculated by computing the trajectories of a group of electrons through the various regions of the gyrocon. The electrons initially are distributed in a disk, and each electron represents a fixed amount of charge. The initial conditions may be set for an ideal beam, or electron data from an electron gun program may be used. The usual configuration of the gyrocon is an input drift space, a deflection cavity, the second drift-space/bender region, the output cavity and a collector drift space. The solenoid fields and the space-charge fields are calculated in each region, and the deflection or output-cavity fields are added in the appropriate regions. The trajectories are integrated with time as the independent variable, and separate time steps for the drift and rf regions may be used. The major assumptions are that the effects of the apertures in the deflection and output cavities are neglected, and that the space-charge rods can adequately represent the beam. 
The equations discussed in Sec. II above are used to find the electron trajectories, and we define the rf drive power and the electronic and overall efficiencies to complete the analysis. The electrons gain energy from the deflection-cavity fields that are due to beam-loading effects; thus the total rf drive power is the sum of the losses and the beam-loading power. The beamloading power is evaluated at the first time step, after all of the electrons have left the input cavity. The electronic efficiency of the output cavity is evaluated in a similar manner immediately after the last electron leaves the output cavity. The actual power, the rf gain, and the overall dc-to-rf conversion efficiency are now defined.

The resulting equations complete the mathematical model of the gyrocon, and any gyrocon of the type illustrated in Fig. 50 may be analyzed. Time steps corresponding to $1 \mathrm{~mm}$ in the drift regions, and $0.5^{\circ}$ of the $\mathrm{rf}$ cycle in the rf regions, generally are used in the computations, and a typical run takes $15 \mathrm{~s}$ on the CDC 7600 computer. The code is named GYRO1.

\section{B. Electron Dynamics}

The most serious problem in the radial gyrocon is the aberration arising from the bender solenoid. Figure 52 shows the projections of the trajectories on to the $r z$ plane for the prototype. The aberration is evident by the large radial deflection of the inner electrons and by the reduced radial deflection of the outer electrons. This aberration could result in a large spread in axial velocities as the beam traverses the output cavity and, therefore, result in lower efficiency. Another problem that is caused by the bender-field aberration is shown in Fig. 53, (the top view of the trajectories of the prototype gyrocon). The inner electrons execute more cyclotron motion than the outer electrons and this difference increases the azimuthal size of the beam. Both effects are caused because the beam traverses the fringe field of the bender solenoid and these fields decrease quickly with distance. Gyrocons should be designed to produce a beam minimum in the bender region to obtain the highest possible efficiency.

The focus coils at the start of the output cavity reduce the angular spread in the $r z$ plane, but also increase the azimuthal extent of the beam. In this case the beam in the output cavity is about $53^{\circ}$ wide in azimuth, compared to about $110^{\circ}$ in time for the bunch width in the best klystrons. It is this better bunching that results in such high electronic efficiencies 


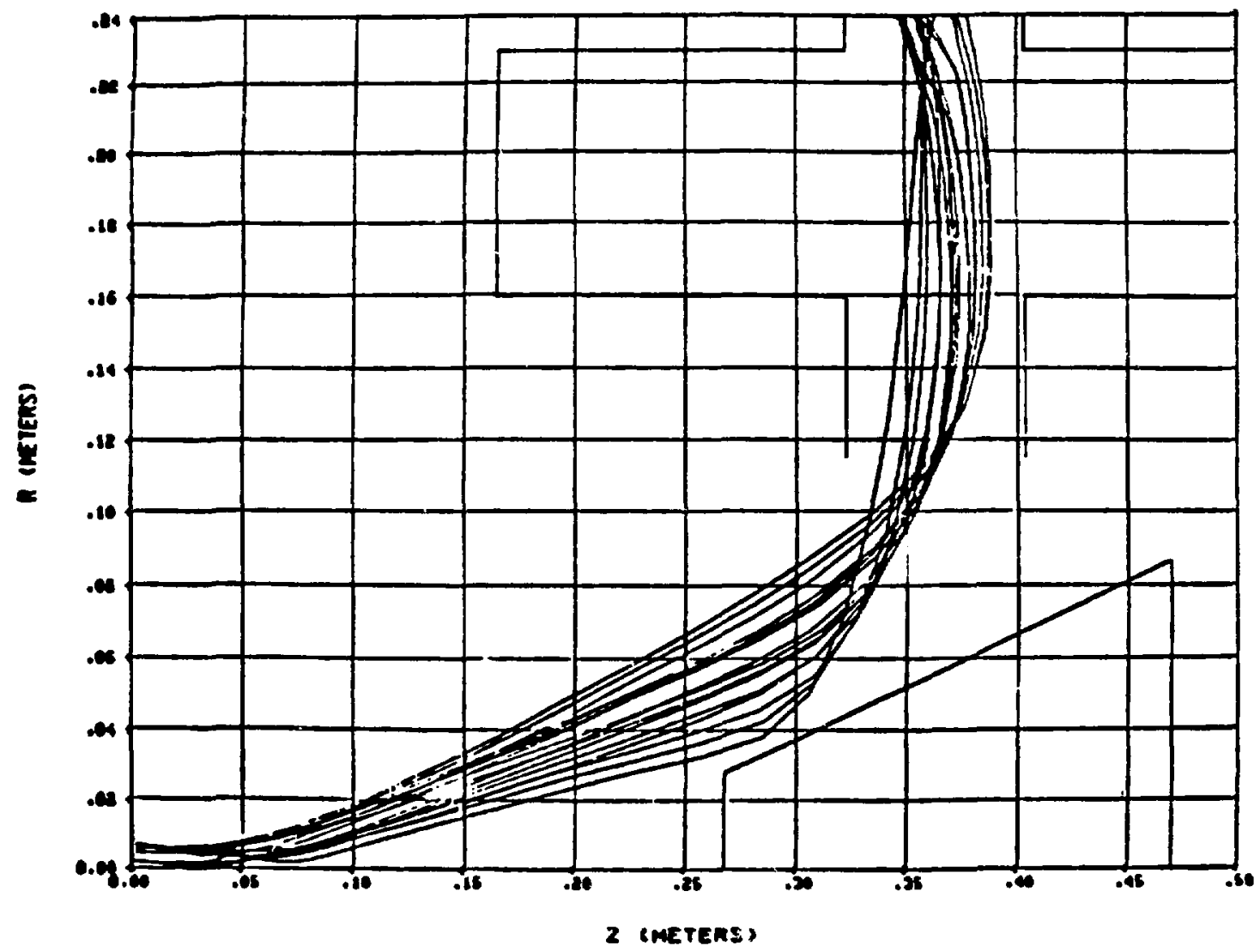

Fig. 52.

Trajectories projected on the $r z$ plane for the LASL gyrocon.

for the gyrocon. The bunching improves with the beam voltage (at constant beam current), and we have obtained beam widths of $20^{\circ}$ at $500 \mathrm{kV}$.

The beam spreads from space-charge forces and from any nonuniform radial velocities acquired in the deflection cavity. The space charge is relatively high in the prototype gyrocon; thus the optimum deflection angle is large and the $r f$ gain is rather low. The $r f$ forces in the output waveguide produce a net change of angular momentum in the negative direction, because the $r f$ angular momentum is in the opposite direction. The polarity of all the coils becomes important. It is essential that the cyclotron motion given to the beam by the bender be in the same direction as the power flow in the output waveguide; in the prototype calculations, we found that the efficiency could be raised by a small amount if the initial cyclotron motion given to the beam by the first-focus coil opposes the cyclotron motion produced by the bender. 


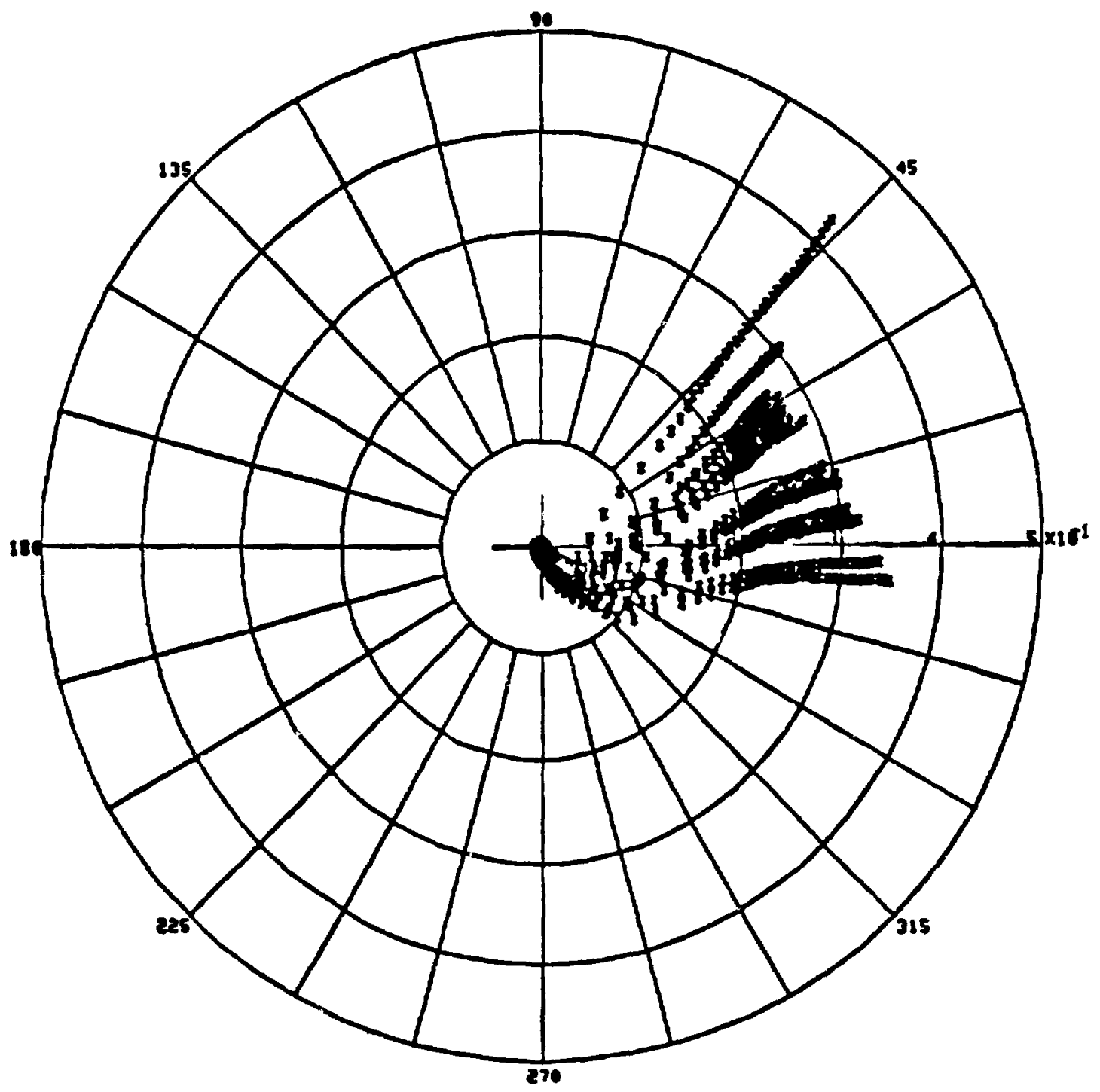

Fig. 53.

Electron trajectories projected on to the $r \phi p l a n e$.

An additional pair of coils was put in the collector region to control the electron dynamics between the output cavity and collector.

Figure 54 shows another projection of the trajectories onto the $r z$ plane and also includes contour lines of the magnetic field. The maximum magnetic fields anywhere on a trajectory are less than $0.02 \mathrm{~T}$ for the prototype gyrocon. Higher voltage devices need stronger magnetic fields, but the requirements are rather modest. 


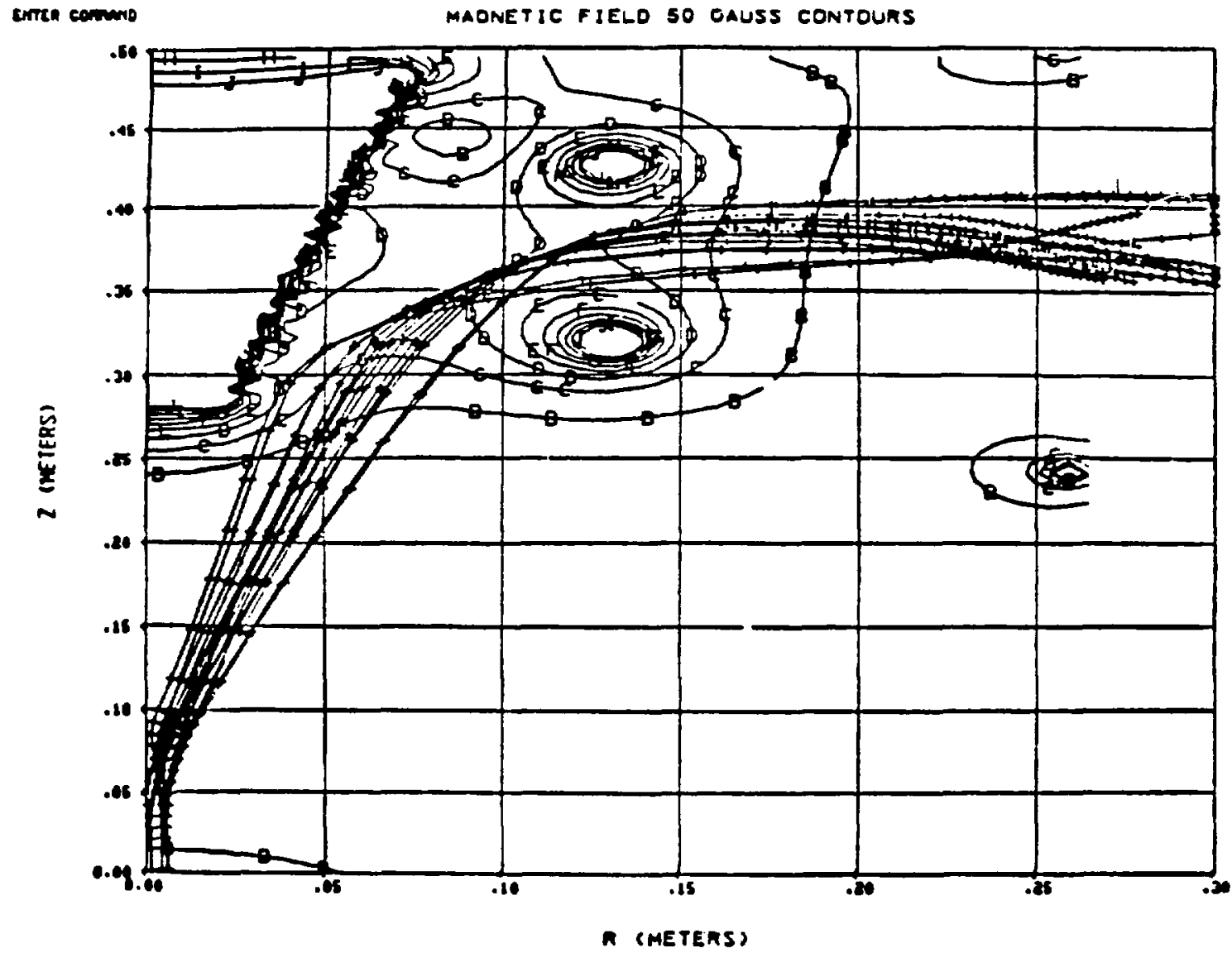

Fig. 54 .

Magnetic contours and electron trajectories on the $r z p l a n e$ for the prototype gyrocon.

C. Limits to Radial Gyrocon Performance

Although the calculated efficiencies of the prototype devices are very impressive, the next question to be answered concerns the power and frequency regions over which such excellent performance may be expected. It is obvious that to stop an electron beam of fairly high voltage, above $50 \mathrm{kV}$ or so, many $k$ ilowatts of $r f$ are required. It also takes several kilowatts to deflect such a beam; thus, output powers of tens or hundreds of kilowatts are required for high overall efficiency.

The ideal method of optimizing gyroron performance is to thoroughly explore the entire parameter space to find the maxima, as functions of beam voltage, current, frequency, and beam initial size. The parameter space is 
too large to conduct an effective search, because there are over 60 variables associated with the coils alone, and another dozen or more rf and geometric variables. The authors have made hundreds of computer runs trying to accomplish this rather difficult task. We present the optimum results obtained so far, with the warning that for any particular case there may be a higher efficiency solution somewhere in the parameter space.

The major parameter search has been directed toward obtaining the highest overall efficiency as a function of beam voltage for several values of beam power. Table VII shows the results for a frequency of $450 \mathrm{MHz}$ and an initial beam radius of $1.0 \mathrm{~cm}$. These results show that the overall efficiency increases with an increase in the beam power. For any particular beam power, there is an optimum beam voltage, and this voltage also increases with beam power. The gyrocon performs better as the beam power is increased, but the power density, or peak electric field allowable in the output gap, establishes a maximum power limitation. The Soviet authors ${ }^{73}$ suggest a maximum electric field of $10 \mathrm{MV} / \mathrm{m}$ in the $\mathrm{cW}$ case and $50 \mathrm{MV} / \mathrm{m}$ for the pulsed case. The peak fields in the output cavities of the gyrocons in Table VII are all below $10 \mathrm{MV} / \mathrm{m}$, with the exception of the 2000-kV, 5-A case.

A series of calculations also were made to determine the variation of the overall efficiency as the beam voltage was varied and as the beam current was adjusted to keep the perveance constant. Table VIII shows the results of these computations. The overall efficiency increased under these conditions as the beam voltage was increased for the calculations, at a perveance of $3 \times 10^{-8}$. The overall efficiency is generally higher at the larger perveance of $3 \times 10^{-7}$, but the beam was more difficult to control because of the large space-charge forces. Some runs were made with a beam perveance of $1 \times 10^{-6}$, but the overall efficiency was low because of the large beam size at the output cavity. The authors estimate that the optimum beam perveance is within a factor of two of $3 \times 10^{-7}$. The gyrocon is thus a low-perveance device and must be operated with high voltages to achieve the highest values of efficiency. The fundamental reason for the better performance at low perveance is the reduction in space-charge effects that signific antly reduces the beam size, and the reduced beam size allows a higher electronic efficiency. The Soviet authors ${ }^{73}$ have shown that, for a rectangular output waveguide, in the limit of zero beam size and zero space charge, one $c$ an always find waveguide parameters that yield $100 \%$ electronic conversion efficiency. 

100-kW Dene Power

\begin{tabular}{|c|c|c|c|c|c|c|c|c|c|c|c|c|c|}
\hline $\begin{array}{r}50 \\
100 \\
200 \\
500 \\
1000\end{array}$ & $\begin{array}{l}2.00 \\
1.00 \\
0.50 \\
0.20 \\
0.10\end{array}$ & $\begin{array}{r}11 \\
11 \\
14 \\
14 \\
8\end{array}$ & $\begin{array}{l}1.05 \\
1.05 \\
0.90 \\
1.50 \\
2.10\end{array}$ & $\begin{array}{r}8.7 \\
5.9 \\
4.2 \\
10.3 \\
17.3\end{array}$ & $\begin{array}{l}14 \\
14 \\
14 \\
10 \\
14\end{array}$ & $\begin{array}{r}7.0 \\
7.0 \\
12.0 \\
16.0 \\
18.0\end{array}$ & $\begin{array}{l}1.10 \\
1.80 \\
1.30 \\
0.60 \\
0.30\end{array}$ & $\begin{array}{l}285 \\
310 \\
325 \\
330 \\
320\end{array}$ & $\begin{array}{l}0.8872 \\
0.9026 \\
0.4803 \\
0.1026 \\
0.0312\end{array}$ & $\begin{array}{r}10.6 \\
28.3 \\
23.1 \\
2.0 \\
1.7\end{array}$ & $\begin{array}{l}0.7187 \\
0.5852 \\
0.2389 \\
0.0750 \\
0.0126\end{array}$ & $\begin{array}{r}78.1 \\
61.9 \\
24.9 \\
8.3 \\
1.5\end{array}$ & $\begin{array}{r}8.9 \\
10.6 \\
5.9 \\
0.8 \\
0.1\end{array}$ \\
\hline \multicolumn{14}{|c|}{ 300-kH Deen Power } \\
\hline $\begin{array}{r}50 \\
100 \\
200 \\
500 \\
1000\end{array}$ & $\begin{array}{l}6.00 \\
3.00 \\
1.50 \\
0.60 \\
0.30\end{array}$ & $\begin{array}{l}11 \\
11 \\
16 \\
14 \\
10\end{array}$ & $\begin{array}{l}1.30 \\
1.30 \\
1.00 \\
1.65 \\
1.00\end{array}$ & $\begin{array}{r}26.9 \\
12.8 \\
8.2 \\
13.3 \\
4.1\end{array}$ & $\begin{array}{r}17 \\
14 \\
9 \\
10 \\
9\end{array}$ & $\begin{array}{r}7.0 \\
7.0 \\
8.0 \\
18.0 \\
24.0\end{array}$ & $\begin{array}{l}1.15 \\
2.15 \\
3.50 \\
4.10 \\
1.80\end{array}$ & $\begin{array}{l}280 \\
305 \\
325 \\
320 \\
315\end{array}$ & $\begin{array}{l}0.8582 \\
0.9615 \\
0.9626 \\
0.7384 \\
0.1945\end{array}$ & $\begin{array}{l}11.6 \\
33.5 \\
62.8 \\
96.4 \\
19.5\end{array}$ & $\begin{array}{l}0.7522 \\
0.8149 \\
0.7332 \\
0.3943 \\
0.1277\end{array}$ & $\begin{array}{r}245.9 \\
254.9 \\
226.0 \\
125.1 \\
38.8\end{array}$ & $\begin{array}{r}8.9 \\
19.8 \\
27.5 \\
9.4 \\
9.4\end{array}$ \\
\hline \multicolumn{14}{|c|}{ 1000-kH Deen Power } \\
\hline $\begin{array}{r}100 \\
200 \\
500 \\
1000\end{array}$ & $\begin{array}{r}10.00 \\
5.00 \\
2.00 \\
1.00\end{array}$ & $\begin{array}{r}10 \\
18 \\
11 \\
9\end{array}$ & $\begin{array}{l}1.80 \\
0.70 \\
1.80 \\
2.40\end{array}$ & $\begin{array}{l}42.1 \\
10.7 \\
15.5 \\
25.9\end{array}$ & $\begin{array}{l}14 \\
10 \\
10 \\
09\end{array}$ & $\begin{array}{r}7.0 \\
10.0 \\
16.0 \\
25.0\end{array}$ & $\begin{array}{l}2.05 \\
3.40 \\
5.85 \\
6.70\end{array}$ & $\begin{array}{l}315 \\
315 \\
315 \\
310\end{array}$ & $\begin{array}{l}0.9135 \\
0.9559 \\
0.9381 \\
0.6863\end{array}$ & $\begin{array}{r}45.5 \\
61.5 \\
188.8 \\
149.2\end{array}$ & $\begin{array}{l}0.8329 \\
0.8848 \\
0.7378 \\
0.3979\end{array}$ & $\begin{array}{l}868.0 \\
894.4 \\
749.3 \\
408.2\end{array}$ & $\begin{array}{l}20.6 \\
83.0 \\
48.3 \\
15.9\end{array}$ \\
\hline \multicolumn{14}{|c|}{ 3000-kH Been Power } \\
\hline $\begin{array}{r}100 \\
200 \\
500 \\
1000\end{array}$ & $\begin{array}{r}30.00 \\
15.00 \\
6.00 \\
3.00\end{array}$ & $\begin{array}{r}6 \\
12 \\
12 \\
9\end{array}$ & $\begin{array}{l}3.60 \\
1.80 \\
1.70 \\
2.40\end{array}$ & $\begin{array}{r}113.2 \\
61.7 \\
20.3 \\
24.1\end{array}$ & $\begin{array}{l}14 \\
16 \\
13 \\
14\end{array}$ & $\begin{array}{r}7.0 \\
11.0 \\
16.0 \\
18.0\end{array}$ & $\begin{array}{l}2.05 \\
3.00 \\
5.60 \\
7.30\end{array}$ & $\begin{array}{l}310 \\
310 \\
310 \\
315\end{array}$ & $\begin{array}{l}0.8514 \\
0.9269 \\
0.9413 \\
0.7459\end{array}$ & $\begin{array}{r}45.5 \\
77.3 \\
238.5 \\
968.3\end{array}$ & $\begin{array}{l}0.8058 \\
0.8830 \\
0.8560 \\
0.4197\end{array}$ & $\begin{array}{l}2508.7 \\
2703.3 \\
2585.3 \\
1269.4\end{array}$ & $\begin{array}{r}22.2 \\
43.8 \\
127.5 \\
51.6\end{array}$ \\
\hline \multicolumn{14}{|c|}{ 10000-kH Been Power } \\
\hline $\begin{array}{r}200 \\
500 \\
1000 \\
2000\end{array}$ & $\begin{array}{r}30.00 \\
20.00 \\
10.00 \\
5.00\end{array}$ & $\begin{array}{l}20 \\
15 \\
13 \\
12\end{array}$ & $\begin{array}{l}1.50 \\
2.00 \\
2.90 \\
4.80\end{array}$ & $\begin{array}{r}684.5 \\
101.9 \\
63.9 \\
114.6\end{array}$ & $\begin{array}{l}13 \\
18 \\
13 \\
13\end{array}$ & $\begin{array}{r}7.0 \\
14.0 \\
18.0 \\
19.0\end{array}$ & $\begin{array}{r}4.00 \\
5.90 \\
10.40 \\
17.80\end{array}$ & $\begin{array}{l}320 \\
310 \\
305 \\
310\end{array}$ & $\begin{array}{l}0.8231 \\
0.9426 \\
0.9644 \\
0.8647\end{array}$ & $\begin{array}{r}167.2 \\
359.5 \\
824.7 \\
2464.9\end{array}$ & $\begin{array}{l}0.7548 \\
0.8975 \\
0.8763 \\
0.6112\end{array}$ & $\begin{array}{l}8064.8 \\
9067.3 \\
8619.3 \\
6181.6\end{array}$ & $\begin{array}{r}11.8 \\
88.9 \\
137.9 \\
53.9\end{array}$ \\
\hline
\end{tabular}


TABLE VIII

DEPENDECE OF EPPICIENCY OA PERVEANCE FOA THE

450-MHz GROCON WITH 1-CE InITIAL BEAM RADIUS

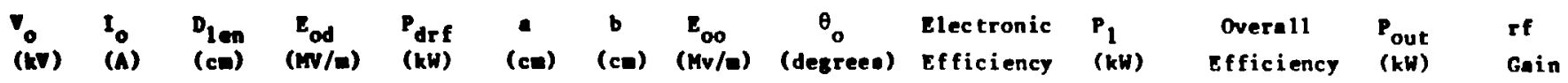

Pervenuce of $3 \times 10^{-8}$

\begin{tabular}{|c|c|c|c|c|c|c|c|c|c|c|c|c|c|}
\hline 100 & 0.95 & 13 & 1.6 & 12.3 & 13 & 20 & 2.3 & 305 & 0.9406 & 35.9 & 0.4976 & 53.3 & 4.3 \\
\hline 200 & 2.68 & 15 & 2.3 & 52.9 & 13 & 25 & 2.7 & 310 & 0.8843 & 50.3 & 0.7195 & 424.1 & 8.0 \\
\hline 500 & 10.61 & 16 & 2.9 & 169.9 & 13 & 31 & 5.5 & 300 & 0.8499 & 230.2 & 0.7843 & 4277.2 & 28.5 \\
\hline 1000 & 30.00 & 11 & 2.5 & 62.0 & 16 & 35 & 9.6 & 310 & 0.8850 & 1917.2 & 0.8194 & 24633.0 & 397.3 \\
\hline 2000 & 84.85 & 8 & 7.8 & 400.7 & 21 & 44 & 16.1 & 300 & 0.8838 & 3796.4 & 0.8594 & 146190.0 & 364.8 \\
\hline
\end{tabular}

Perveance of $3 \times 10^{-7}$

\begin{tabular}{|c|c|c|c|c|c|c|c|c|c|c|c|c|c|}
\hline 100 & 9.49 & 13 & 1.3 & 42.3 & 15 & 21 & 2.3 & 315 & 0.9166 & 40.8 & 0.8363 & 829.0 & 19.6 \\
\hline 200 & 26.83 & 10 & 2.5 & 114.2 & 15 & 26 & 2.9 & 305 & 0.8872 & 66.9 & 0.8565 & 4694.0 & 41.1 \\
\hline 500 & 106.07 & 10 & 6.4 & 1226.0 & 19 & 27 & 8.0 & 325 & 0.9128 & 639.9 & 0.8804 & 47769.0 & 39.0 \\
\hline 1000 & 300.0 & 19 & 3.8 & 7085.0 & 13 & 29 & 10.5 & 325 & 0.8653 & 805.3 & 0.8427 & 258785.0 & 36.5 \\
\hline 2000 & 049.53 & 13 & 12.2 & 27571.0 & 27 & 45 & 17.1 & 290 & 0.8127 & 5171.2 & 0.7967 & 1374029.0 & 49.8 \\
\hline
\end{tabular}


A third series of calculations were made to determine the high-frequency limitation of the radial gyrocon. The beam's initial radius was held at $1 \mathrm{~cm}$, the beam voltage and current were held at $86 \mathrm{kV}$ and $9.0 \mathrm{~A}$ for these calculations; Table IX is a summary of the results. The beam size becomes larger, compared to the wavelength, as the frequency is increased and the electronic efficiency decreases. At $1200 \mathrm{MHz}$, the highest overall efficiency calculated for this type of gyrocon is only $52 \%$, and it should be much easier to design a klystron with a higher efficiency at this and higher frequencies.

\section{THE LASL GYROCON DEMONSTRATION TUBE}

A gyrocon is now under construction at Los Alamos Scientific Laboratory (LASL) to demonstrate the validity of the above theory and to test the gyrocon concept. The frequency was fixed at $450 \mathrm{MHz}$ by programatic requirements for a proton accelerator, and the beam voltage was fixed at $86 \mathrm{kV}$, where a large power supply was available. A magnetic system was designed with POISSON, a magnet-design code, to give a 9-A fairly laminar beam from an existing electron gun (Fig. 55). The trajectories were calculated with Herrmannfeldt's trajectory program. ${ }^{79}$ The four beamlets shown in the figure were further subdivided by a factor of four in azimuth, and a gyrocon was designed to optimize the overall efficiency. An overall efficiency of slightly over $86 \%$ was ach ieved with the parameters listed in Table $x$.

To optimize the gyrocon parameters, generally it is necessary to have the power lost in the output cavity equal to the total deflection power, and the two terms in the total deflection power must be approximately equal. This

TABLE IX

ETECT OF FREQUENCY ON GYROCON PERFORHANCE

$$
\left(v_{0}=86 \mathrm{kV}, I_{0}=9 A, r_{0}=1-\mathrm{ce}\right)
$$

\begin{tabular}{|c|c|c|c|c|c|c|c|c|c|c|}
\hline $\begin{array}{c}f \\
(\mathrm{MHz})\end{array}$ & $\begin{array}{l}D_{1 \text { en }} \\
\text { (ca) }\end{array}$ & $\begin{array}{l}\text { Eod }_{\text {od }} \\
(\boldsymbol{n v} / \mathbf{a})\end{array}$ & $\begin{array}{l}P_{\text {drf }} \\
(k w)\end{array}$ & $\begin{array}{l}E_{\infty} \\
(\mathbf{N} / \mathbf{a})\end{array}$ & $\begin{array}{c}\theta_{0} \\
\text { (degrees) }\end{array}$ & $\begin{array}{l}b-a \\
(c a)\end{array}$ & $\begin{array}{l}\text { Electronic } \\
\text { Efficiency }\end{array}$ & $\begin{array}{c}\text { Overall } \\
\text { Efficiency }\end{array}$ & $\begin{array}{l}P_{\text {out }} \\
\text { (kw) }\end{array}$ & $\begin{array}{c}\text { rf } \\
\text { Gein }\end{array}$ \\
\hline 100 & 4.0 & 2.90 & 269.6 & 2.90 & 335 & 12.0 & 0.7204 & 0.4933 & 514.8 & 1.9 \\
\hline 200 & 7.0 & 2.00 & 503.7 & 1.00 & 330 & 11.0 & 0.9017 & 0.8037 & 662.5 & 13.2 \\
\hline 450 & 8.0 & 2.00 & 30.5 & 1.80 & 310 & 7.0 & 0.9184 & 0.8502 & 682.6 & 22.4 \\
\hline 600 & 7.0 & 2.10 & 28.9 & 2.60 & 315 & 5.0 & 0.9155 & 0.8257 & 662.9 & 22.9 \\
\hline 900 & 6.0 & 1.90 & 20.4 & 2.80 & 280 & 5.0 & 0.8174 & 0.7399 & 587.8 & 28.8 \\
\hline 1200 & 0.0 & 2.30 & 650.1 & 3.40 & 255 & 5.0 & 0.7520 & 0.6429 & $\$ 39.4$ & 8.3 \\
\hline 1500 & 2.0 & 6.00 & 28.9 & 3.25 & 225 & 3.0 & 0.4666 & 0.4130 & 331.6 & 11.5 \\
\hline
\end{tabular}




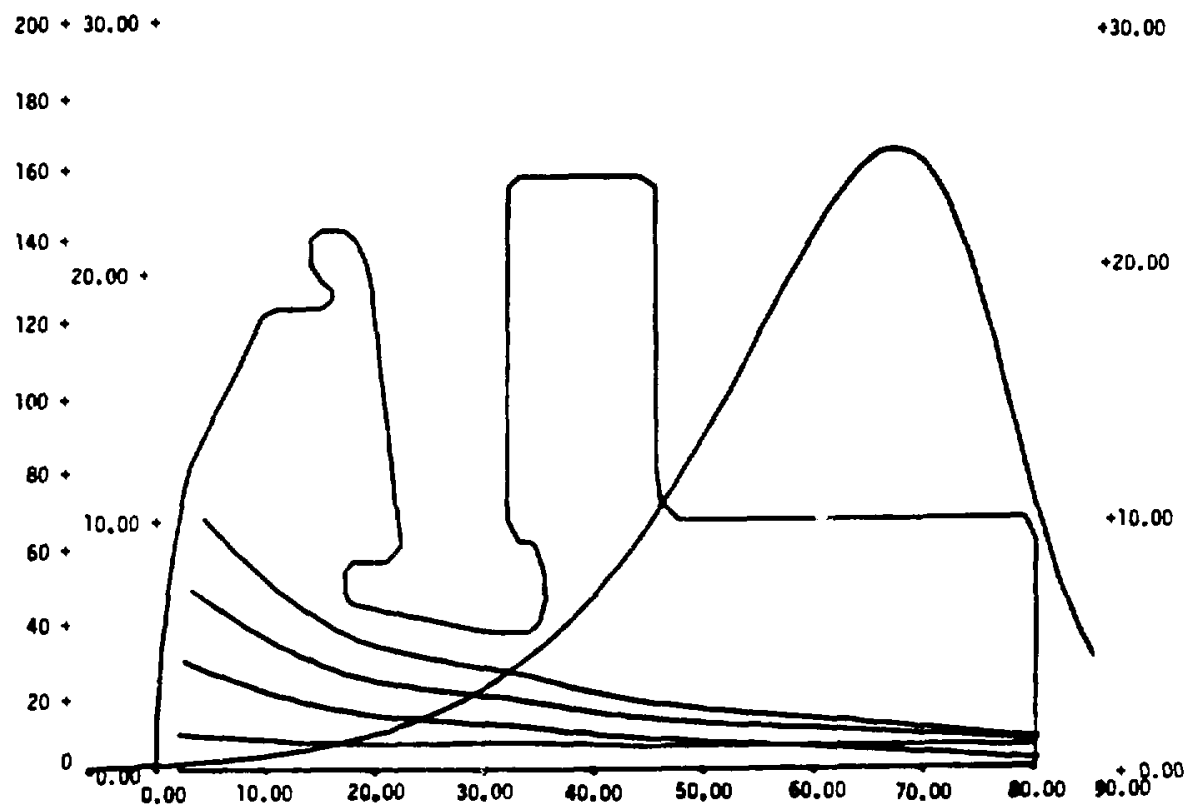

Fig. 55 .

Electron trajectories in the gun region of the LASL gyrocon.

TABLE $X$

MAJOR PARAMETERS OF THE LASL GYROCON

Beam Voltage

$86 \mathrm{kV}$

Beam Current

$9.0 \mathrm{~A}$

Deflection Angle

$13.4^{\circ}$

Def lection Length

$8.0 \mathrm{~cm}$

Deflection Field

$2.0 \mathrm{MV} / \mathrm{m}$

Total Deflection Power

$31.4 \mathrm{~kW}$

First-Focus Coil Ampere Turns

$-1540$

Conical Bender Ampere Turns

14000

Second-Focus Coil Ampere Turns

4000

Output Cavity, i.r.

$16.8 \mathrm{~cm}$

Output Cavity, o.r.

$23.8 \mathrm{~cm}$

Output Cavity Field

$1.80 \mathrm{MV} / \mathrm{m}$

Output Phase

$310^{\circ}$

Electronic Eff ic iency

93.0

Radio-frequency Gain

22.0

Overall Efficiency

86.1

Output Power

$691.9 \mathrm{~kW}$ 
procedure tends to yield a highly efficient but low-gain design. The gain can be increased at the expense of efficiency, but the present device was designed for the highest dc-to-rf conversion efficiency.

The overall design of the prototype gyrocon is completed and some components have been fabricated. The very high-average beam and output powers have made the mechanical design rather difficult. Figures 56 and through 59 show some of the subassemblies and Fig. 60 shows the test stand. The prototype is being designed for $\mathrm{CW}$ operation and will be ready for testing by June 1980.

\section{THE SPHERICAL GYROCON}

It should be possible to improve the overall efficiency of the gyrocon at frequencies into the gigahertz region if the bender can be eliminated. The simplest method of eliminating the bender is to return to the original bermutron idea, but to use a spherical cavity, as shown in Fig. 51. The output cavity is a section of conical waveguide bounded by two concentric spheres, and the analysis is fairly straightforward, although it is complicated to reduce to code. The authors have analyzed this type of output cavity; we will describe the results of the field analysis and then present some computed results which show that the spherical gyrocon is a useful device up to the 2to 3-GHz frequency range.

The output resonator is a section of conical waveguide bounded by two concentric spheres. The fields (in spherical coordinates) are derived, neglecting the effects of the slots. Insertion of the boundary conditions produces a generally nonintegral result, yielding generalized Legendre functions. Power losses and resonator-quality factor are found by a combination of analytical and numerical integration.

A separate computer code, GYROS, has been written to evaluate the field expressions and to analyze the spherical gyrocon. The input parameters are very similar to those used for GYR01, although a static magnetic field was added to the output region in GYROS. This static compensation field is more important in the spherical case than for the radial-style gyrocon. 

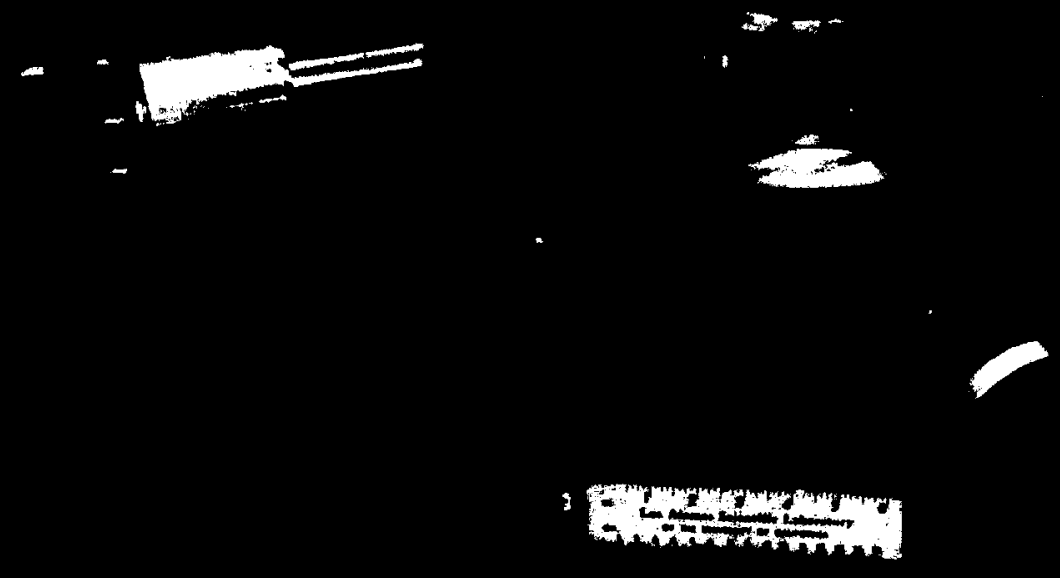


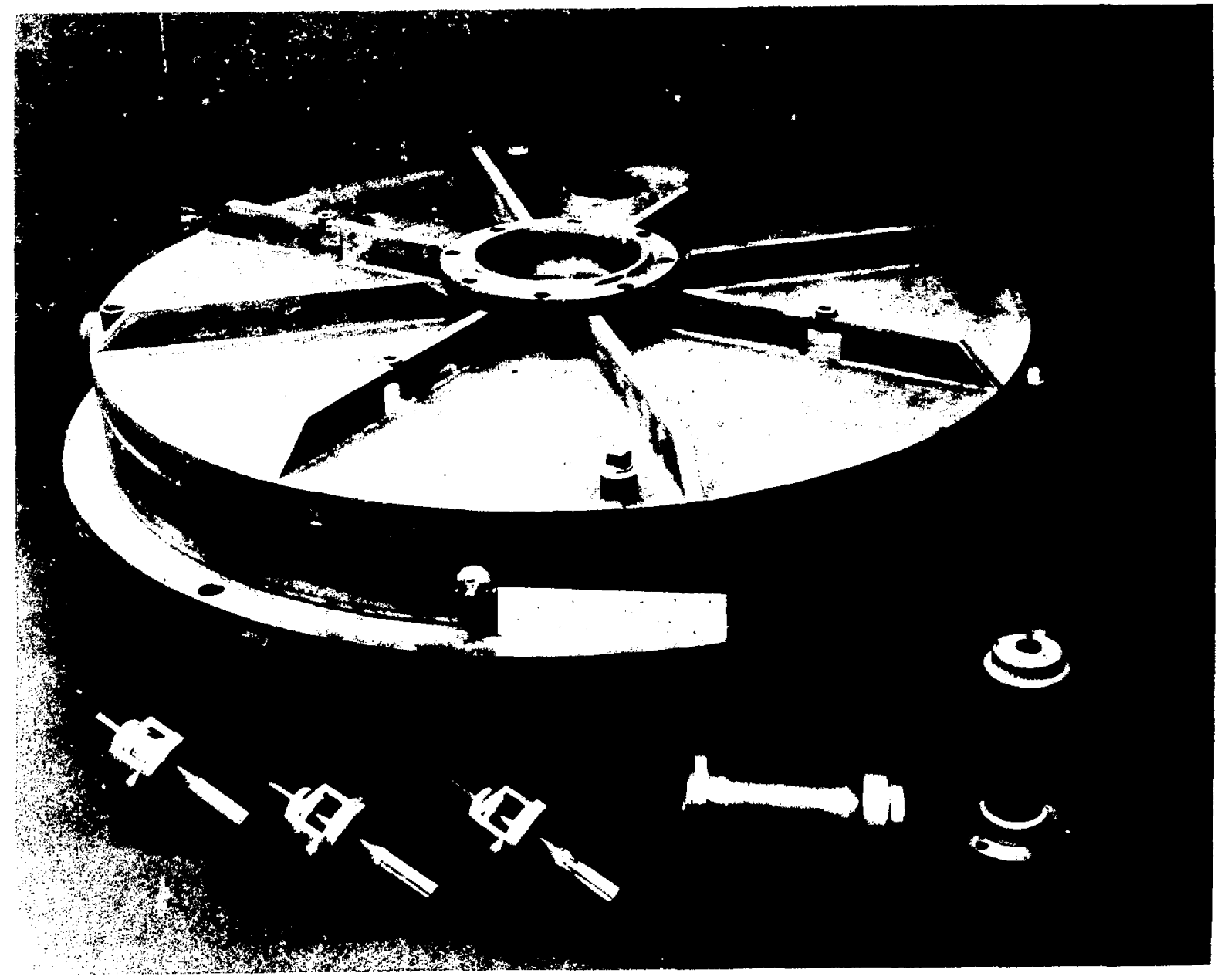

Fig. 57 .

Deflection cavity with input loops and tuners, prior to assembiy. 


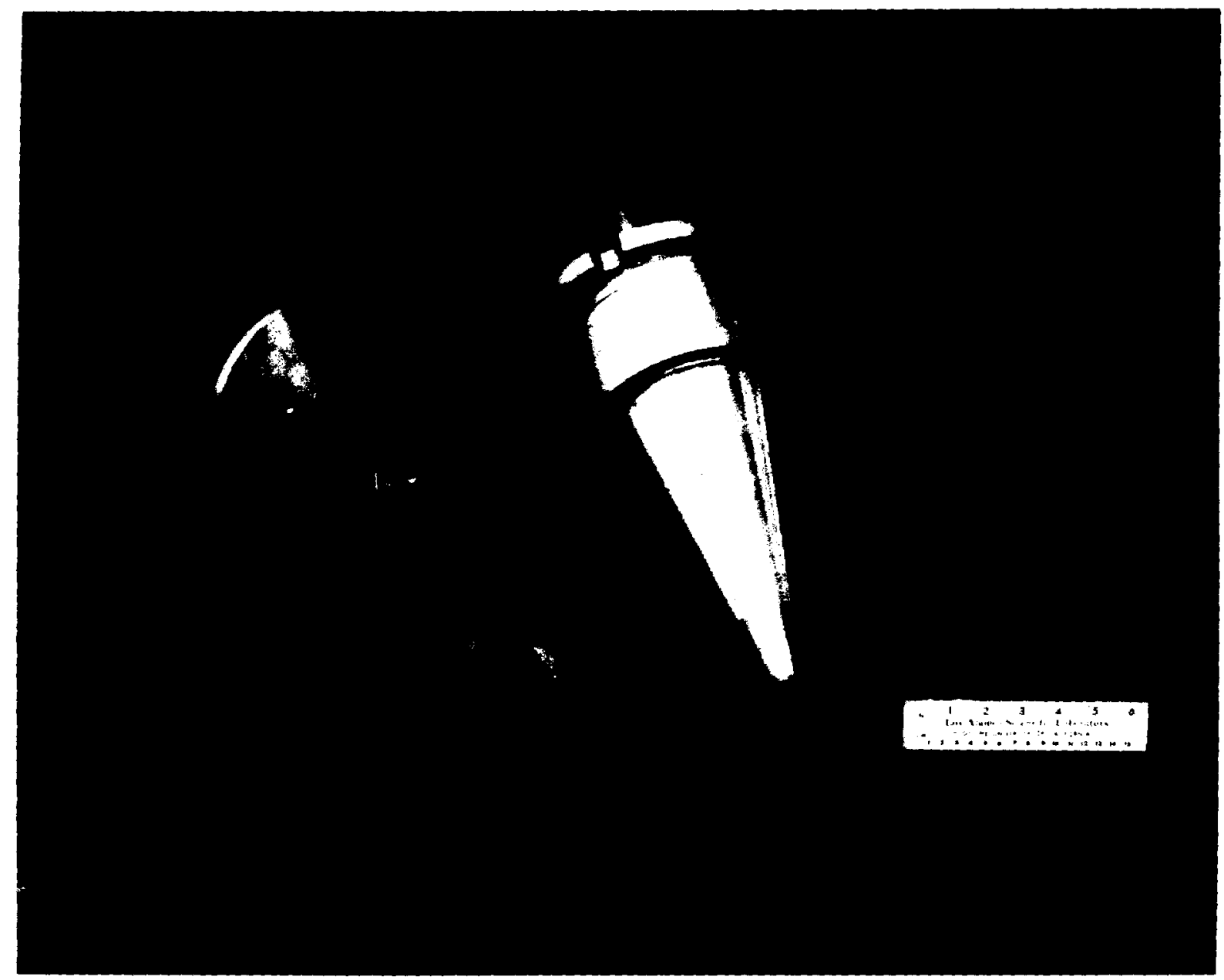

Fig. 58 .

The control bender magnet components. 
$\$$

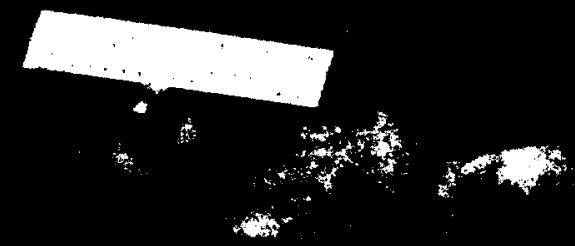

Fig. 59.

The collector assembly. 


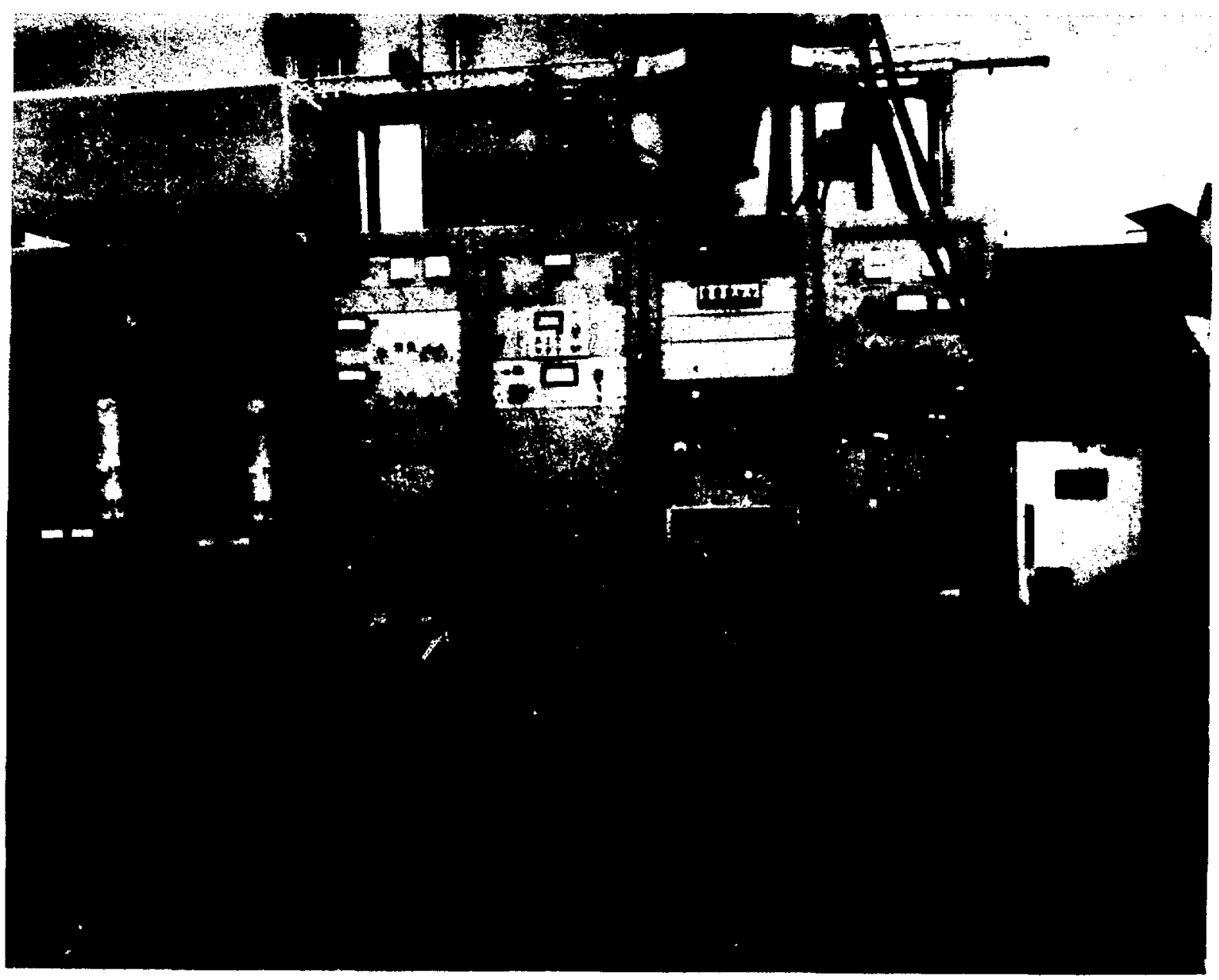

Fig. 60 .

Partial view of grrocon test stand. 


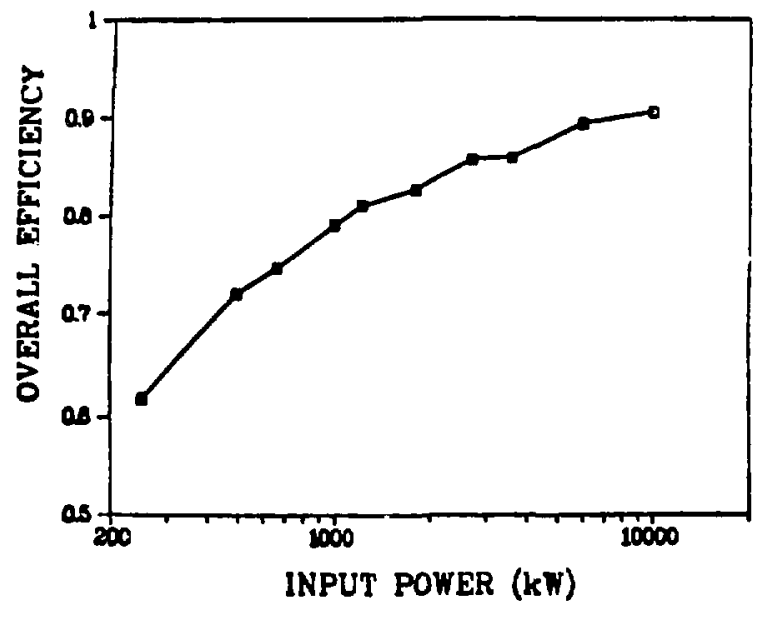

Fig. 61.

Overall efficiency vs dc beam input power for the spherical gyrocon.

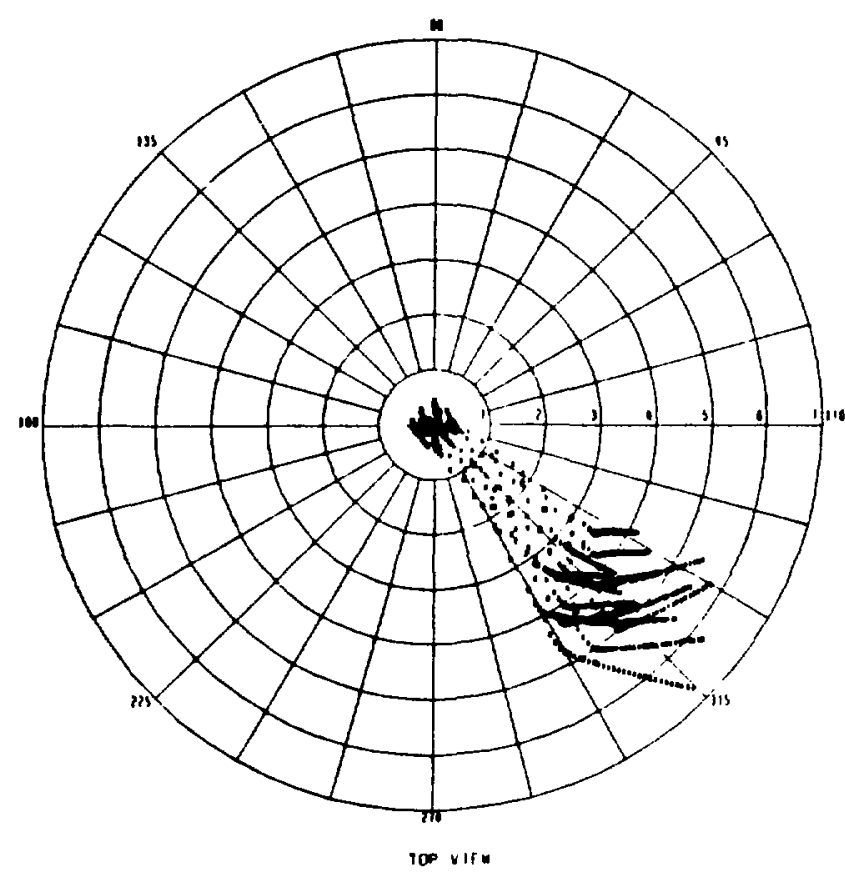

Fig. 62.

Top view of the trajectories for the spherical gyrocon on the ro plane.
We have made a limited parameter search of spherical gyrocon characteristics using the LASL code GYROS. At $2.45 \mathrm{GHz}$ (the solar power satellite frequency) we find increasing overall efficiency with increasing input power (Fig. 61). The microperveance for these runs is about 0.1 , which makes the gyrocon a low-preveance device. The efficiency curve is roughly logarithmic, and electronic efficiencies of over $97 \%$ are predicted for the higher input powers. Output-cavity wall losses, at 1.8-MW input, average about $350 \mathrm{~W} / \mathrm{cm}^{2}$.

Total output-cavity losses are about $172 \mathrm{~kW}$. Figures 62, 63, and 64 show various projections of the path formed by the passage of a single layer of electrons through the device at 1.8-MW input. These also show the output-cavity outline, and the output-cavity beamfocusing fields. The very narrow beam bunching shows clearly on the r-plane projection. The static, magnetic, compensating field is not shown, but is roughly $200 \mathrm{G}$ in the negative theta direction. Overcompensation here seems to produce improved efficiency.

In the 1.8-M simulation, tube parameters include $70-\mathrm{kW}$ drive power, an output-cavity $Q$ 


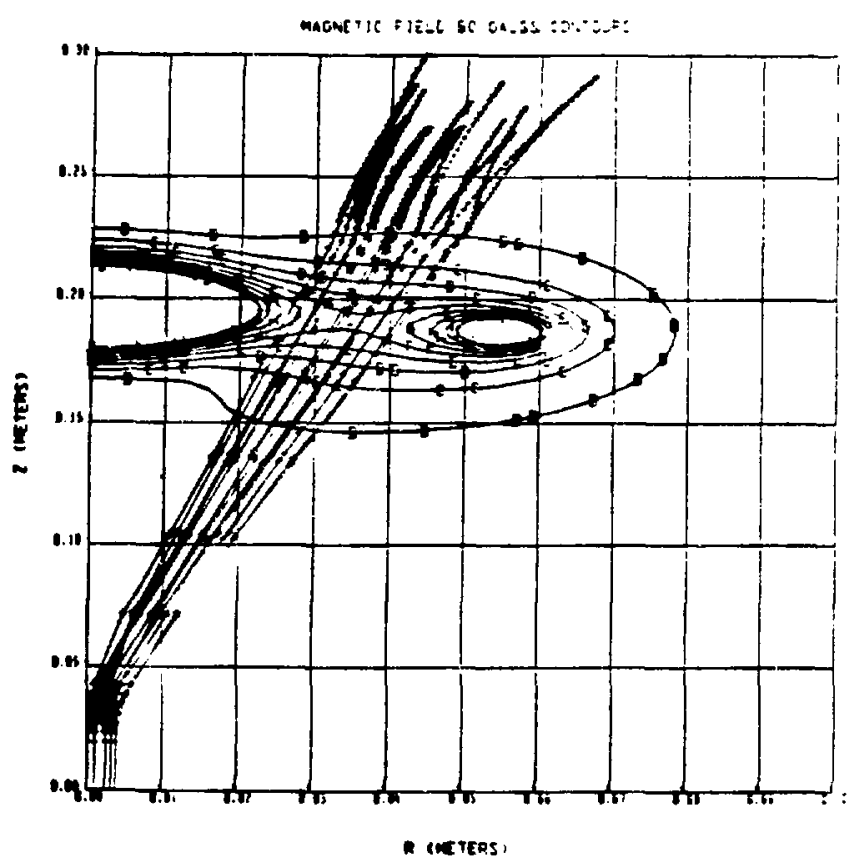

Fig. 63.

Projection of spherical gyrocon trajectories on to the $r z$ plane and 50-G magnetic contours from output-cavity focus coils.

vensus 2

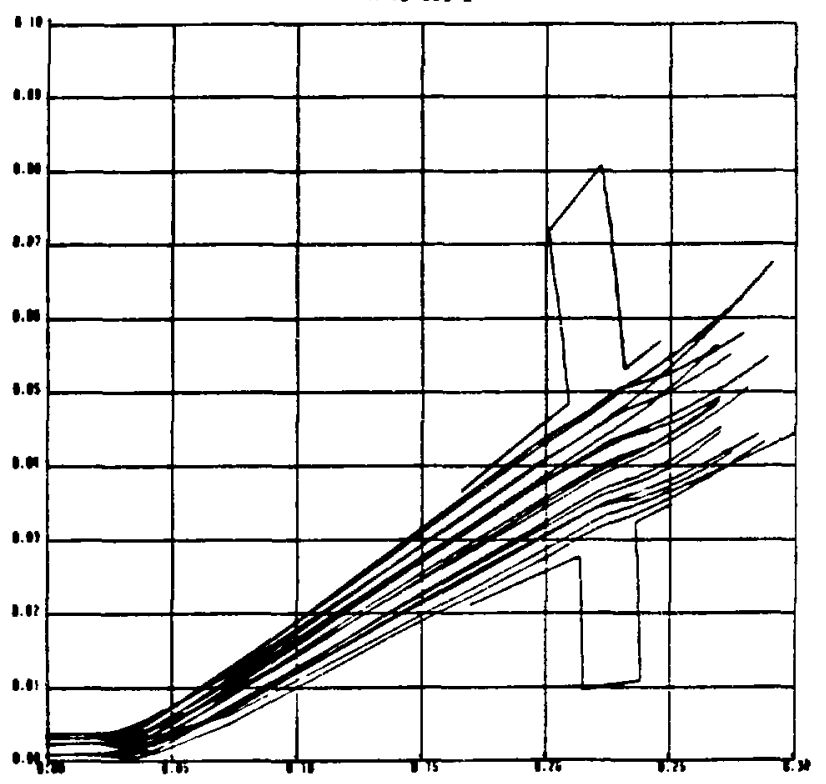

2 (WETERS)

Fig. 64.

Projection of the spherical gyrocon trajectories onto the $r z$ plane with the outline of the output cavity. over 11000 , an initial beam radius of $4 \mathrm{~mm}$, a deflection angle of $11.9^{\circ}$, and an output power of 1.55 MW. Output power is taken from pairs of output lines with internal separations of $90^{\circ}$ in each pair. The phasing of paired outputs is necessary to maintain the correct output-cavity traveling wave.

Also we have made a series of simulations of a tube of 1.8-MW input power at several frequencies.

A graph of over-all efficiency versus frequency shows a slow rise in efficiency as the frequency is lowered. Thus, the sphercial gyrocon is a viable generator into the several-GHz frequency region. The ever-diminishing size of the output cavity would make operation above $3 \mathrm{GHz}$ impossible without heroic cooling techniques.

VI. PROBLEMS REMAINING AND SUGGESTIONS FOR FUTURE WORK

The code-development work at LASL will be directed towards removing some of the assumptions made in the present codes. Work has begun on the estimation of the aperture fringe fields in the output cavity, using both the electrostatic approximation and SUPERFISH output. These fringe fields are expected to be quite 
important above $1 \mathrm{GHz}$, because the cavity slot width becomes an appreciable fraction of a wavelength at these high frequencies.

Both the radial and the spherical style of gyrocon show rather low power gain of $1 \mathrm{c}$ to $20 \mathrm{~dB}$. We have done some work with a developmental version of GYR01 that allows multiple deflection cavities to be analyzed. The second deflection cavity is driven by the beam's modulation current only, just as the intermediate cavities in a klystron. The initial results show an increase in gain of 3 to $5 \mathrm{~dB}$ per cavity; but the overall efficiency remains the same, because some of the $\mathrm{dc}$ beam power is converted to deflection cavity energy. We feel that this type of calculation cannot be performed accurately unless we have a self-consistent cavity field calculation. In this calculation, we would estimate a field amplitude and phase in a deflection or output cavity, then calculate the induced current in the cavity caused by the beam. Next, we would use the known cavity impedance to calculate the field's amplitude and phase. We would relax the solution and trace the particles through the field again. The method is used in klystrons, but it is a bit more complicated in gyrocon applications. This self-consistant analysis would give the cavities' frequency detuning by the bunched beam.

The best performance for the gyrocon family in the 2- to 4-GHz frequency $r$ ange may be possible only by eliminating the deflection system, as well as the bender solenoid. Figure 65 shiws schematically one embodiment of this idea that we call the planar gyrocon. The cathode now must have a rotating emissive area; thus no deflection cavities are required. The plate supply pulls the electron to the output cavity, where the energy extraction takes place. The difficult part of this arrangement is the electron gun. One possible solution to this problem is the inverted Amplitron gun, ${ }^{80}$ which utilizes a slow-wave structure made of secondary emitting materials as the cathode. Another possible solution to the planar gun problem is to use a multipactor electron gun, 81 which in the multipactor region moves in a slow-wave or waveguide structure. Still another possibility is to use a thermionic cathode as part of a waveguide. A traveling wave in the input waveguide could control the electron flow to the output waveguide in much the same manner as the control grid in a triode. Lebacqz, ${ }^{82}$ the originator of this last method, calls this dual-waveguide version of the planar gyrocon a trirotron. At this time, we will not speculate on which embodiment of the 


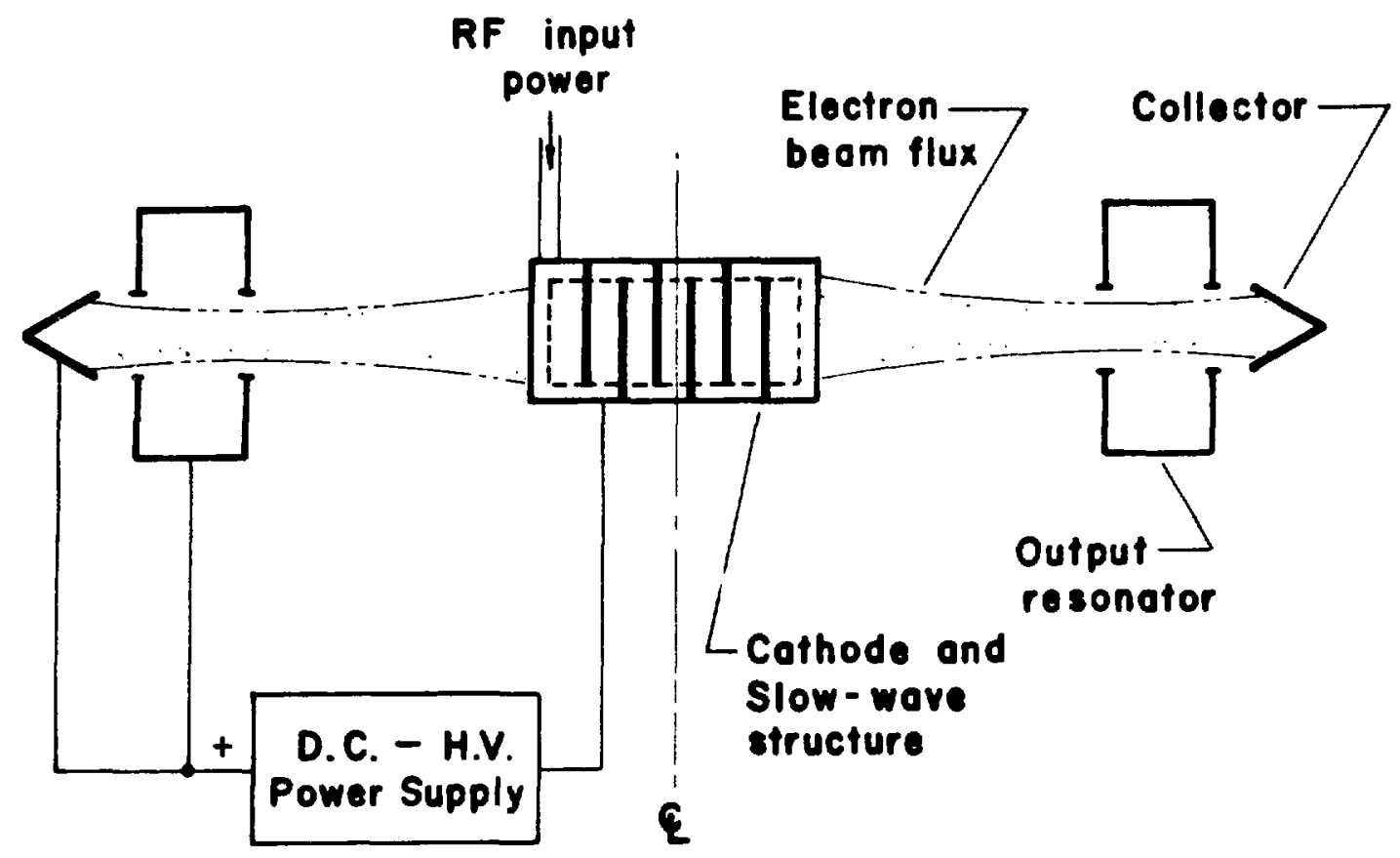

Fig. 65 .

The planar gyrocon.

planar gyrocon will prove optimum, but we feel that at least one of these will be practical as a high-efficiency amplifier in the 2- to 4-GHz $r$ ange. At these high frequencies, it would probably be best to build the planar gyrocon in the inverted configuration with the cathode surrounding the output cavity.

The bandwidth of the gyrocon is very narrow because the $Q$ of the input resonator is about 20000 . The bandwidth of the output cavity is also fairly narrow, but this cavity has a loaded $Q$ of $\sim 1000$. The effects of a mismatch or of frequency deviations from resonance in the output cavity may be analyzed with resonant-ring theory. The output power and high-frequency limitations of the gyrocon arise from the finite size of the beam and the ohmic losses in the output resonator. We estimate that the device can be competitive with klystrons and crossed-field devices up to frequencies of $3 \mathrm{GHz}$. The low-frequency limit to the gyrocon must be rather close to the 181-MHz, 5-MW $\mathrm{Cw}$ Soviet machine, which is $4.25 \mathrm{~m}$ tall and weighs over $5000 \mathrm{~kg}$. The rf gain of the gyrocon is between 10 and $25 \mathrm{~dB}$ for the examples calculated here, but we expect that the supplementary deflection cavities of Fig. 62 should add another 10 to $20 \mathrm{~dB}$. 
The present analysis indicates that the gyrocon amplifier should be considered for multimegawatt, narrow-band microwave systems that operate below approximately $3 \mathrm{GHz}$. In such applications, the excellent dc-to-rf conversion efficiency and the large power available per device should overcome its narrow bandwidth and rather modest gain limitations. If its planar variant can be produced, the gyrocon could be made in a much simpler manner and then it would become a lightweight device that could be useful for high-power airborne or space applications of microwaves. 
$H^{-}$ION SOURCE, INJECTOR, AND ACCELERATOR TEST STAND STUDIES

I. $H^{-}$ION SOURCE AND INJECTOR STUDIES

A. Dudnikov Source Emittance

Emittance measurements of the Dudnikov source were reported previously. ${ }^{83}$ The emittance scanner passes a ribbon of beam through a slit of width $\Delta x$ located at a distance $x$ from the beam center. This ribbon is deflected electrically so that ions with a divergence $x^{\prime}$ pass through a rear slit with acceptance $\Delta x^{\prime}$ into the scanner's Faraday cup. Thus the cup current is proportional to the current density $j$ in phase space

$j=\partial^{2} i / \partial x \partial x^{1}$

The scanner then plots all of the phase space area in which $j$ is greater than the threshold value $j_{T}$. The threshold $T$ is defined by $T=j_{T} / j_{1}$, where $j_{1}=$ the maximum value of $j$. The fraction of the current within a given threshold $T$ was calculated to be

$i_{T}=i_{0}\left(\varepsilon_{T}-\varepsilon_{1}+\varepsilon_{T} \ln \varepsilon_{0} / \varepsilon_{T}\right) /\left(\varepsilon_{0}-\varepsilon_{1}\right)$

where $\varepsilon_{0}$ is the zero threshold emittance, $\varepsilon_{1}$ is the cutoff emittance. This fraction was calculated using previous data ${ }^{83}$ for a 100-mA beam. For $i / i_{0}=0.7$ the emittances are 0.05 and $0.07 \pi \mathrm{cm} \cdot \mathrm{mrad}$ in the two planes; and if the beam were collimated to these values, the brightness $B$ would be $3 \mathrm{~A} /(\mathrm{cm} \cdot \mathrm{mrad})^{2}$, substantially below the value calculated using emittances at half threshold. Because several questionable assumptions were used in the calculation, we plan to remeasure emittance, using beam collimators and measuring the total current vs threshold.

B. Rotating Ion Source (RIS)

1. RIS-1 Results and Critical Questions. The goal of the RIS program is to demonstrate that the fixed-electrode Dudnikov source, transformed to a circular, rotating geometry, can produce $100 \mathrm{~mA}$ of $\mathrm{H}^{-} \mathrm{dc}$ for a discharge voltage $\leqslant 100 \mathrm{~V}$ and a discharge current $<100 \mathrm{~A}$. The proof-of-principle demonstration lies in showing that RIS-1 can make $100 \mathrm{~mA}$ of $\mathrm{H}^{-}$during pulsed operation at $100 \mathrm{~V}$ and $100 \mathrm{~A}$. In addition, the RIS-1 discharge must be confined by the magnetic field to a region along the periphery of the electrode 
wheel of about $1 \mathrm{~cm}$, demonstrating that a cooled version of the RIS can operate at $<10 \mathrm{~kW}$ of discharge power and produce $100 \mathrm{~mA}$ of $\mathrm{H}^{-} \mathrm{dc}$.

The best RIS-1 results are given in Table XI. Note that the switch from the rotating anode to the fixed anode significantly improved the RIS-l performance, particularly for pulsed operation.

Several questions remain. First, replacing the rotating anode with the fixed anode gave much improved source performance; we still must show that the rotating anode can be incorporated into a successful source design. Operation with the fixed anode may not be possible for $100 \mathrm{~mA}$ of $\mathrm{H}^{-} \mathrm{dc}$ because of excessive anode heating. Second, RIS-1 operates at $180 \mathrm{~V}$ dc and $100 \mathrm{~V}$ pulsed discharge voltage, whereas the Dudnikov source operates near $50 \mathrm{~V}$, both pulsed and dc; we do not understand this difference between the two sources. A significant improvement in the power efficiency would result if we lowered this voltage to the Dudnikov source value. Third, the RIS-1 $\mathrm{H}^{-}$efficiency decreases as the discharge current increases. Finally, we have not shown that the beam quality of RIS-1 is comparable to that of the Dudnikov source.

2. RIS Design and Improvements. In developing an ion source to produce $100 \mathrm{~mA}$ of $\mathrm{H}^{-}$at $100 \%$ duty factor, the additional complication of rotating electrodes was added because we found that the cathode surface had a heat load of $\sim 7 \mathrm{~kW} / \mathrm{cm}^{2}$, an order of magnitude top high for continuous operation.

The average-power density onto the rotating electrode surfaces is reduced by confining the discharge to a narrow region near the emission slit, through

\section{TABLE XI}

MEASURED RIS-1 PERFORMANCE COMPARED TO THE DESIGN GOALS

\begin{tabular}{|c|c|c|c|c|c|c|}
\hline & \multicolumn{2}{|c|}{$\begin{array}{l}\text { Rotating Cathode } \\
\text { and Rotating Anode }\end{array}$} & \multicolumn{2}{|c|}{$\begin{array}{l}\text { Rotating Cathode } \\
\text { and Fixed Anode }\end{array}$} & \multicolumn{2}{|c|}{ Design Goals } \\
\hline & $\underline{d c}$ & Pulsed & $\underline{d c}$ & Pulsed & $d c$ & Pulsed \\
\hline Discharge & & & & & & \\
\hline $\begin{array}{l}\text { Voltage (V) } \\
\text { Discharge }\end{array}$ & 220 & 300 & 177 & 88 & 100 & 100 \\
\hline Current (A) & 1 & 90 & 1.5 & 100 & 1 & 100 \\
\hline$I_{H^{-}}(m A)$ & 0.5 & 10 & 1.5 & 40 & 1 & 100 \\
\hline
\end{tabular}


proper adjustment of the discharge magnetic field. The average electrode power density is reduced by the ratio of the length of the discharge region $(\sim 1 \mathrm{~cm})$ to the circumference of the electrodes $(\sim 43 \mathrm{~cm})$, or about 40 to 1 . Because there is much to learn at low-power levels, cooling was not incorporated on RIS-1 (Fig. 66).

3. RIS-1 Parametric Studies. We constructed a special anode, containing only two 2-mm-wide by 5 -mm-long slots located $180^{\circ}$ apart (Fig. 67a) to verify

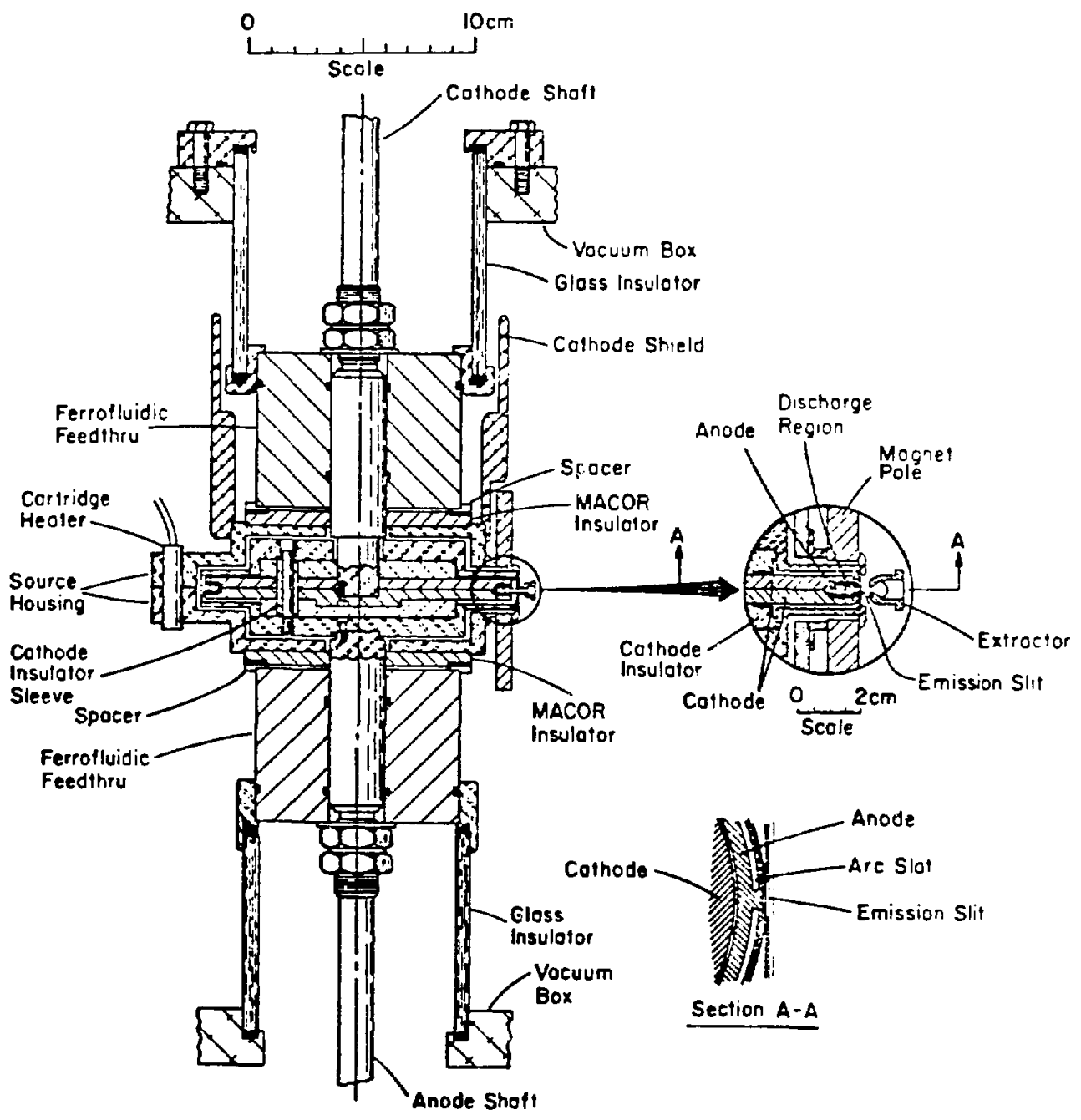

Fig. 66 .

Improved design of the RIS-1 source. 
that the magnetic field confines the discharge. We used arc pole pieces with $1-\mathrm{cm}$ by $1-\mathrm{cm}-\mathrm{square}$ faces (Fig. $67 \mathrm{~b}$ ). The magnetic field was varied between 0.08 and $0.15 \mathrm{~T}(\mathrm{Fig}, 67 \mathrm{c})$. It was possible to reduce the width of the discharge region to $2.5 \mathrm{~mm}$ at a magnetic field of $0.075 \mathrm{~T}$.

When the RIS is run in the pulsed mode with a fixed anode, the variation in $\mathrm{H}^{-}$current and $\mathrm{H}^{-}$production efficiency $\eta^{-}$with magnetic field is very weak. The maximum $I_{H^{-}}(27 \mathrm{~mA})$, the maximum $n^{-}\left(0.27 \times 10^{-3}\right)$, and

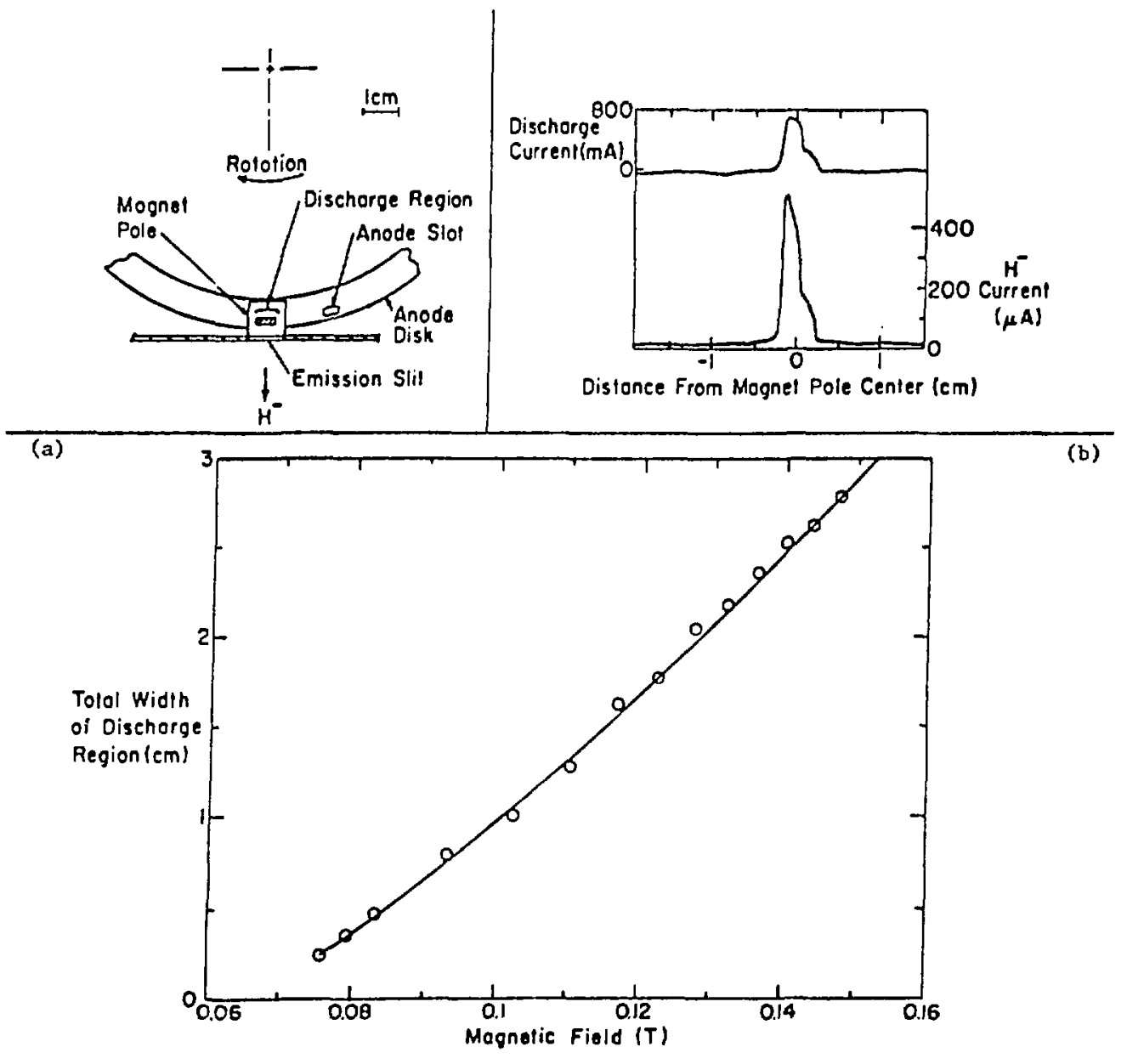

(c)

Fig. 67.

(a) The anode and magnet arc poles used to measure the width of the discharge region as a function of magnetic field strength.

(b) The discharge current (top) and $\mathrm{H}$ - current (bottom) as a function of the location of the 5-mm long arc slot.

(c) The total width of the discharge region vs the discharge magnetic field strength. 


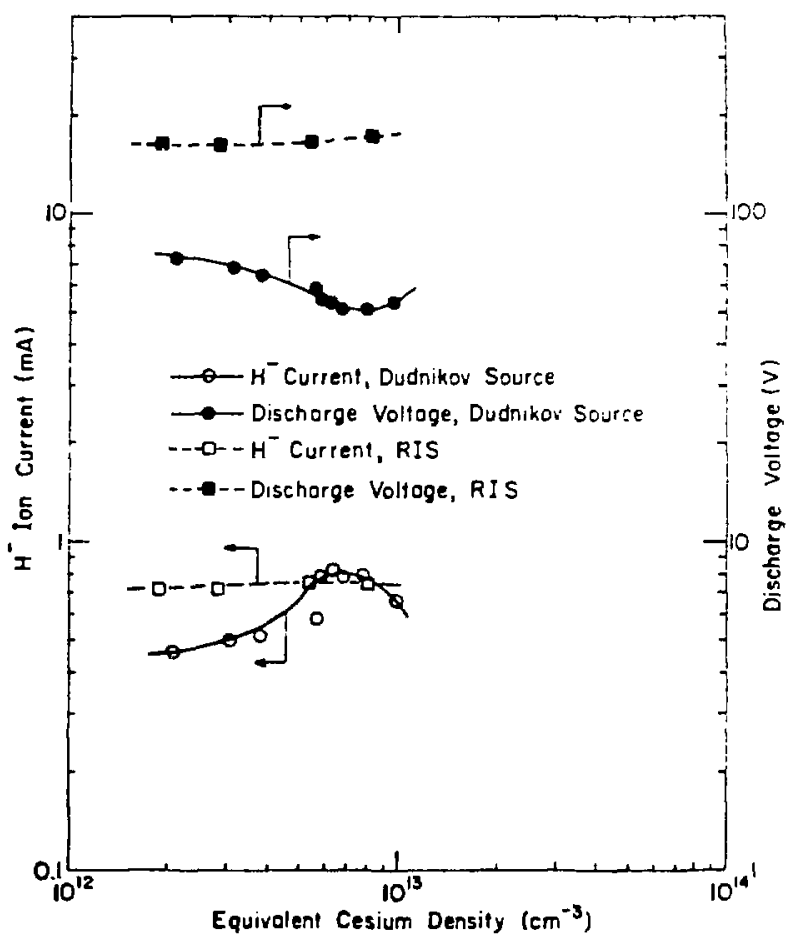

Fig. 68 .

$\mathrm{H}^{-}$ion current and discharge voltage as a function of equivalent cesium density in the source for dc operation of the Dudnikov (circles) and RIS (squares) ion sources. the minimum discharge voltage (80 v) occurred at a magnetic field of $0.1 \mathrm{~T}$ for a 100-A discharge current.

The $\mathrm{I}_{\mathrm{H}^{-}}$and discharge voltage were measured as a function of cesium flow (Fig. 68). The range of cesium flow necessary for proper operation of the Dudnikov source has been covered, with similar results for the Dudnikov and RIS-1 sources.

Feeding $\mathrm{H}_{2}$ gas into the source housing opposite the discharge region is satisfactory for dc operation. However, for pulsed operation, RIS-1 only operates stably at discharge currents below $\sim 20 \mathrm{~A}$. We calculated that at the higher discharge currents the discharge can pump the $\mathrm{H}_{2}$ gas away in a few microseconds, thus causing instabilities. To verify this postulate, a capillary tube with a 0.4-mm-i.d.

hole was directed into the discharge region through the emission slit and was used as the $\mathrm{H}_{2}$ gas feed. We then obtained quiet operation at discharge currents of $80 \mathrm{~A}$. We next replaced the rotating anode with a fixed anode and fed the $\mathrm{H}_{2}$ gas directly into the arc slot as in the Dudnikor source. This change allowed pulsed operation at discharge currents up to $120 \mathrm{~A}$. When we used the fixed-anode arrangement, we obtained 1-ms-long $\mathrm{H}^{-}$pulses of $40 \mathrm{~mA}$.

We experimented with the geometry of the RIS-1 discharge region. We increased the width of the arc slot from $2 \mathrm{~mm}$ to $4 \mathrm{~mm}$ and observed no change in the $\mathrm{H}^{-}$output. Recently, the effect of the anode web thickness on the $\mathrm{H}^{-}$output of the Dudnikov source was studied. In the Dudnikov source this distance is $1 \mathrm{~mm}$; in RIS-1, $2 \mathrm{~mm}$. For a 1-mm spacing the Dudnikov source produced $130 \mathrm{~mA}$ of $\mathrm{H}^{-}$pulsed current; for a 2-mm spacing, nnly $60 \mathrm{~mA}$. Reducing this spacing to $1 / 2 \mathrm{~mm}$ raised the pulsed $\mathrm{H}^{-}$current to $160 \mathrm{~mA}$, but 
eliminating it resulted in only $65 \mathrm{~mA}$. We expect that a redesigned RIS-1 discharge region with a spacing of $1 / 2 \mathrm{~mm}$ between the arc slot and the emission slit will result in $100 \mathrm{~mA}$ of pulsed $\mathrm{H}^{-}$current, our RIS-1 design goal.

C. Cesium Flow Studies

1. SIG Design Improvements. We improved the surface ionization gauge (SIG) described in the last report ${ }^{84}$ and used it to study the role of cesium in producing $\mathrm{H}^{-}$beams in our surface-plasma sources (SPS). ${ }^{85}$ A copper cylindrical collector surrounds a tungsten ribbon filament, and the ribbon filament is exposed to the source discharge through a rectangular slit in the SIG collector. Neutral cesium atoms emanating from the source enter the SIG through this slit and strike the filament. The filament operates above the temperature at which $\sim 100 \%$ surface ionization of the $\mathrm{Cs}^{0}$ to $\mathrm{Cs}^{+}$occurs. A small negative bias (typically $60 \mathrm{~V}$ ) between the collector and filament ensures collection of the surface-ionized $\mathrm{Cs}^{+}$. The flux of cesium atoms emanating from the source $c$ an be calculated with the knowledge of the solid angle subtended by the SIG entrance slit, the spatial distribution of cesium atoms emanating from the source, and the measured SIG collector (or filament) current.

The cesium flow from the ion sources can be measured while they are operating, but without extracting the $\mathrm{H}^{-}$beam.

We now examine the question of the SIG response time. An estimate of the lifetime $\tau$ of the fractional monolayer $\theta$ on the filament is $\tau=\theta \sigma / v$, where $\theta$ is the monolayer coverage, $\sigma$ is the density of a single monolayer $\left(4.8 \times 10^{14}\right.$ atoms $\left./ \mathrm{cm}^{2}\right)$, and $v$ is the desorption rate. For $\theta=0.002$, $\nu=9.8 \times 10^{16}$ ions $/ \mathrm{cm}^{2} \mathrm{~s}$ at $1500 \mathrm{~K}$, so $\tau=10 \mu \mathrm{s}$. If $\theta \sigma / \nu$ is calculated from Taylor and Langmuir's dat $\alpha^{86}$ and plotted vs $\theta$, the extrapolated $\tau$ at $\theta=0$ intercept is $9 \mu \mathrm{s}$. If this were the limiting contribution to the time resolution of the gauge, then this response time could be shortened considerably by raising the filament temperature.

The measured time response of the electronics is $15 \mu \mathrm{s}$. The resulting overail time response of the SIG plus electronics is $-20 \mu \mathrm{S}$ and considerably shorter than the time broadening $(-165 \mu \mathrm{s})$ caused by the thermal velocity of the cesium atoms escaping from the source. 


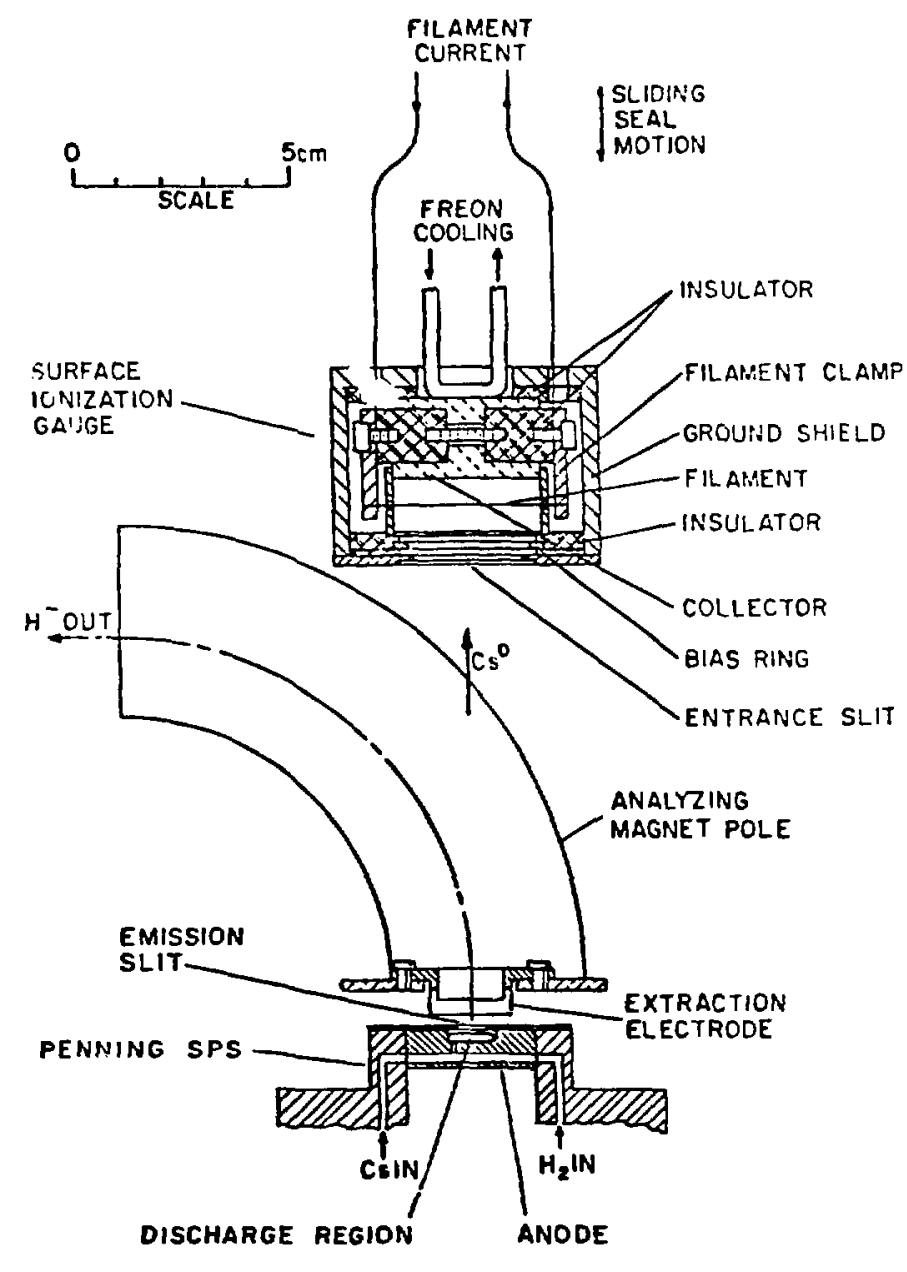

Fig. 69.

Placement of the SIG for measuring the cesium flow from the Dudnikov source. A sliding seal allows the distance between the SIG and the source to be varied from $6 \mathrm{~cm}$ to $21 \mathrm{~cm}$.
2. Cesium Flow Measure-

ments for the Dudnikov and RIS

Ion Sources. We used the improved SIG to measure the $\mathrm{Cs}^{0}$ flow rate through the emission slit of the Dudnikov source $^{85}$ and the RIS; the results were published in the proceedings of the 1979 Partic le Accelerator Conference. 85

The SIG was located as shown in Fig. 69. A $1-\mathrm{mm}$ by 25-mm hole machined in the SIG ground shield serves as the entrance slit to the SIG.

Because Bel'chenko, et al. ${ }^{87}$ reported that cesium is trapped by the discharge for pulsed operation of their magnetron SPS, we devised a way to momentarily interrupt the $d c$ discharge to look for the trapping effect in our Penning SPS for dc operation. We modified the pulse so that the 1-A discharge current was driven to 0 within $10 \mu \mathrm{s}$ for $3 \mathrm{~ms}$ at a repetition rate of $7-1 / 2 \mathrm{~Hz}$.

When the discharge is on, the cesium flow from the source is decreased by a factor of 2.8 from the flow when the source discharge is off. For dc operation we think there is close to an equilibrium cesium concentration on the cathode (unlike the pulsed case where there is a nonequilibrium concentration). The reduction in cesium flow during the discharge arises from ionization and subsequent trapping of the cesium atoms in the discharge. When the discharge is momentarily extinguished, the cesium ions are quickly neutralized and the cesium flow increases. This cesium flow appears to be governed by cesium 
desorption from all of the interior surfaces of the source, instead of just the active cathode surface, as in the pulsed case.

The discharge voltage and the magnetically analyzed $\mathrm{H}^{-}$current were measured as a function of the equivalent cesium density (Fig. 68) for constant discharge current $(1 \mathrm{~A})$ and constant hydrogen flow rate $\left(16 \mathrm{~atm} \mathrm{~cm}^{3} / \mathrm{min}\right)$ for the Dudnikov source. Also shown are the discharge voltage and the unanalyzed $\mathrm{H}^{-}$current as a function of the equivalent cesium density for constant discharge current $(1 \mathrm{~A})$ and the hydrogen flow rate $\left(39 \mathrm{~atm} \mathrm{~cm}^{3} / \mathrm{min}\right.$ ) for the RIS.

The RIS measurements suggest that the R.IS discharge voltage and $\mathrm{H}^{-}$ current depend very weakly on the cesium density, as long as it is high enough

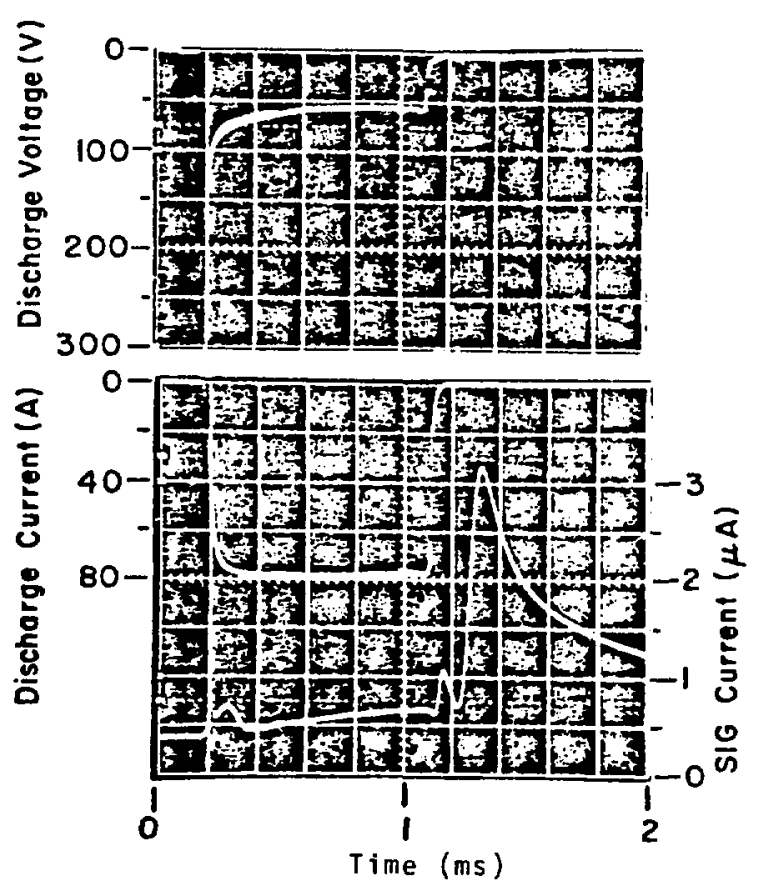

Fig. 70.

Discharge voltage (upper trace), discharge current (middle trace), and SIG current (lower trace) for pulsed operation of the Dudnikov source. The pulse length is $1 \mathrm{~ms}$; the repetition rate, $15 \mathrm{~Hz}$. The cesium pulse occurs 200 us after the discharge is shut off. Approximately $30 \%$ of the SIG current during the discharge pulse and the small peak $\sim 50 \mu \mathrm{S}$ after the discharge pulse is caused by background particles and/or photons from the discharge. for the source to operate in the high-current mode. Only a $5 \%$ variation in discharge voltage and a $4 \%$ variation in $\mathrm{H}^{-}$current occur for a factor of 4 variation in equivalent cesium density. However, the RIS discharge voltage is $170 \mathrm{~V}$, compared with $51 \mathrm{~V}$ for the Dudnikov source. The reason for this discharge-voltage difference is not understood. We used the fixed anode instead of the rotating anode to make the RIS measurements.

Figure 70 shows the discharge voltage, discharge current, and the SIG current for pulsed operation of the Dudnikov souce with the $\mathrm{Ti}+\mathrm{Cs}_{2} \mathrm{Cr}_{2} \mathrm{O}_{7}$ mixture located in a cavity in the anode. Even though the cesium that is desorbed and sputtered from the source cathodes is almost exclusively neutral, the cesium atoms are quickly ionized in the source discharge and returned to the cathode, creating a nonequilibrium of cesium atoms on 
the cathode surface. When the discharge is extinguished, the cesium atoms are thermally desorbed in a short burst, forming the pulse that is detected with the SIG. The SIG current shows that during the discharge the cesium flow fromi the source remains approximately constant. The cesium pulse occurs $\sim 200 \mu \mathrm{s}$ after the discharge ends. The $\mathrm{Cs}^{0}$ emission then decays to the steady-state flow before the next pulse.

By changing the distance of the SIG from the emission slit, the time the cesium burst arrives at the SIG is changed. The discharge voltage and the magnetically analyzed, pulsed $\mathrm{H}^{-}$yield were measured as a function of not for the Dudnikov source, where the height of the cesium burst is proportional to not (I.C. 3 below). During these measurements, the discharge current ( $85 \mathrm{~A})$ and hydrogen gas-flow rate $\left(40 \mathrm{~atm} \mathrm{~cm}^{3} / \mathrm{min}\right)$ were held constant. The product not was varied by using the same independently controlled $2 \mathrm{Ti}+\mathrm{Cs}_{2} \mathrm{Cr}_{2} \mathrm{O}_{7}$ oven used for the dc Dudnikov source measurements. As is true for dc operation, the discharge voltage drops and the $\mathrm{H}^{-}$current increases with increasing nst, until a minimum in voltage and a maximum in $\mathrm{H}^{-}$current are reached. Further increase in not results in a slight rise in voltage and a drop in $\mathrm{H}^{-}$current. The maximum end-of-pulse $\mathrm{H}^{-}$current occurs for a value of $n \delta t=8 \times 10^{9}$ atoms $\mathrm{s} / \mathrm{cm}^{3}$. The average cesium consumption rate at the optimum $\mathrm{H}^{-}$current is $1 \mathrm{mg} / \mathrm{h}$.

3. Cesium Pulse Theory. A simple model, which assumes that the flux of cesium atoms from the source is the same as that from a slit in an ideal oven containing particles with a Maxwellian velocity distribution, can explain the observed time variation of the cesium flow from the source during pulsed operation. The cesium coverage of the cathodes probably is not in thermodynamic equilibrium with the vapor in the source when the discharge is on, because of ionization-enhanced transport of the cesium to the cathodes. When the discharge is extinguished, a brief burst of cesium is desorbed from the discharge-heated cathodes, thus reestablishing equilibrium. Only a small fraction of these atoms effuse through the emission slit. Most are rapidly readsorbed on the cooler surfaces in the source.

The flux of atoms from a slit in an ideal oven containing particles with a Maxwellian velocity distribution is 88

$d^{3} N / d A d v d t=\frac{n}{\sqrt{\pi}}(m / 2 k T)^{3 / 2} v^{3} \exp \left(-m v^{2} / 2 k T\right)$, 
where $N=$ number of particles leaving the oven,

$A=$ area of emission slit,

$\mathbf{n}=$ density of particles in the oven,

and $T$ is assumed to be the temperature of the source cathode. If the time of emission $\delta t$ is small, and the cesium detector is at a distance $d$ from the source, the flux at the detector is

$d N / d t=d^{4}$ Ast $\left(\frac{f \Omega}{\pi 2}\right) \frac{n}{\sqrt{\pi}}(m / 2 k T)^{3 / 2} t^{-5} \exp \left(-m d^{2} / 2 k T t^{2}\right)$,

where $\Omega=$ the solid angle subtended by the detector. The factor $f$ accounts for the azimuthal variation of the flux in Eq. (20) and is 2.4. The detector signal $\mathrm{dN} / \mathrm{dt}$ is a maximum for

$t_{m}=\sqrt{m d^{2} / 5 k T}$

so that

$d N / d t=(d N / d t)_{m}\left(t_{m} / t\right)^{5} \exp \left\{5 / 2\left[1-\left(t_{m} / t\right)^{2}\right]\right\}$,

where

$(d N / d t)=\frac{f \Omega}{2 \pi} \frac{n A \delta t}{\sqrt{\pi d}}(2 k T / m)(5 / 2 e)^{5 / 2}$.

Equation (23) is $f$ it to an experimental measurement with the Dudnikov source by choosing $t_{m}$ and $(d N / d t)_{m}$ appropriately (Fig. 71). From the value of $t_{m}, T$ is determined to be about $760 \mathrm{~K}$ from Eq. (22), close to the observed cathode temperature. Only the product not is determined by $(\mathrm{dN} / \mathrm{dt})_{\mathrm{m}}$ and this is $1 \times 10^{10} / \mathrm{cm}^{3} \mathrm{~s}$. If $\delta$ is taken to be about $50 \mu \mathrm{s}$, then $n$ is $2 \times 10^{14} / \mathrm{cm}^{3}$. The volume of the discharge region is about $0.1 \mathrm{~cm}^{3}$, so that the number of particles is $2 \times 10^{13}$. This would be equivalent to a change in $\theta$ of 0.09 over the active cathode area of $0.48 \mathrm{~cm}^{2}$. 


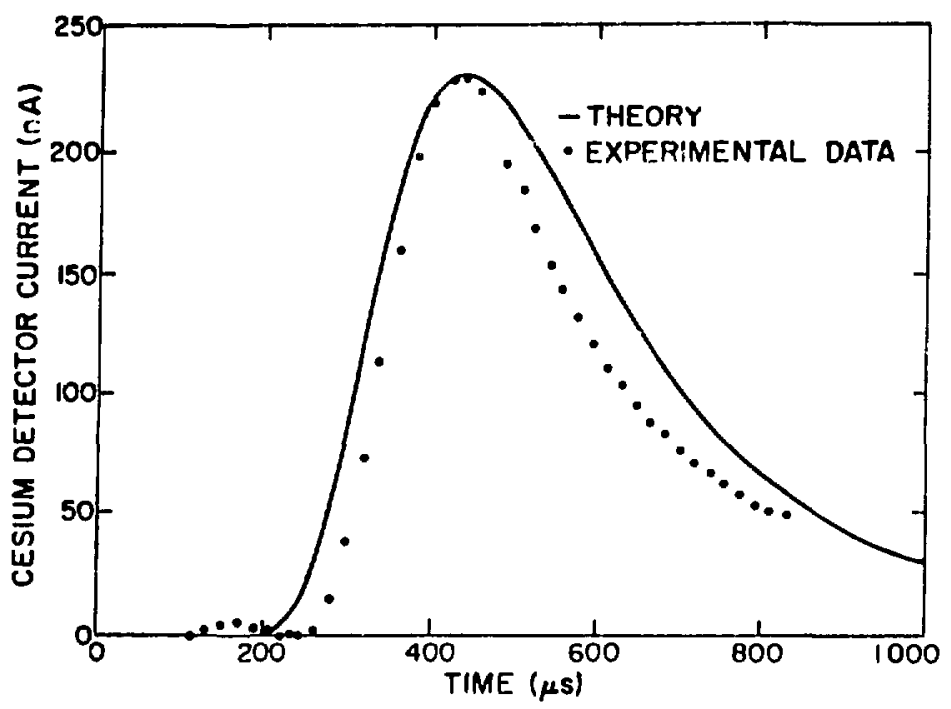

Fig. 71 .

The cesium detector current $\mathrm{dN} / \mathrm{dt}$ vs the time $t$ from the end of the discharge for pulsed operation of the Dudnikov source. The $f$ it (curve) to the experimental measurement (circles) is made by determining $t_{m}$ and $(d N / d t)_{m}$ and then substituting these values into Eq. 23.

4. Evolution of Cesium from the $2 \mathrm{Ti}+\mathrm{Cs}_{2} \mathrm{Cr}_{2} \mathrm{O}_{7}$ Mixture. We know of no published informatior available on the evolution rate of cesium from the $2 \mathrm{Ti}+\mathrm{Cs}_{2} \mathrm{Cr}_{2} \mathrm{O}_{7}$ mixture. We measured cesium evolution rate from the bichromate mixture and found it to be steady for short times, with occasional i-ps-long spikes. The temperature change needed to double the cesium evolutiol, rate from the fresh, outgassed $2 \mathrm{Ti}+\mathrm{Cs}_{2} \mathrm{Cr}_{2} \mathrm{O}_{7}$ mixture at the normal oven operating temperature $\left(\sim 430^{\circ} \mathrm{C}\right)$ is $16^{\circ} \mathrm{C}$. At $430^{\circ} \mathrm{C}$ to evolution rate from a $0.65-\mathrm{g}$ mixture was $0.5 \mathrm{mg} / \mathrm{h}$, which is the cesium cun-i. tion rate of the Dudnikov source for the optimum jroduction $c i \cdots ! ?$ beam (I.C.2 above).

D. Dipole Magnet Studies

Last year we determined empirically that the dominant abt: $\because$ sis of the $90^{\circ}$ magnet were given by

$\delta^{2} \phi_{f}=A_{1} \theta_{f} y_{f}+A_{2} x_{f} y_{f}$

where the final coordinates are $x_{f}, \theta_{f}$ and $y_{f}, \phi$. Construction of a magnet with nearly zero sextupole component did not lead to a low value of $\delta^{2} d_{f}$. 
We analyzed the magnet using the second-order coefficients calculated in the program TRANSPORT. ${ }^{89}$ Agreement between the calculations and experiment for the $A_{1}$ coefficient is good, and for the $A_{2}$ coefficient there is qualitative agreement. These calculations show that aberrations caused by the exit fringe field of ine magnet are large and can be minimized but not eliminated with an optimal sextupole component in the bending field. These results are published in the 1979 Particle Accelerator Conference proceedings. ${ }^{90}$

\section{E. The 20-keV Heam Transport}

A study of the $20-\mathrm{keV} \mathrm{H}^{-}$beam line from the extractor slit to the accelerating-column injection plane has been completed, using the first-order ionoptics search routines in TRANSPORT. ${ }^{89,91}$ Because of the asymmetric emission slit $(1 \mathrm{~cm}$ by $0.05 \mathrm{~cm})$ and the requirement that an approximately circular beam be injected into the accelerating column, a system of three magnetic elements consisting of an $n \neq 0$ dipole and two quadrupole magnets is proposed.

Our TRANSPORT calculations did not consider space charge for an imperfectly neutralized $\mathrm{H}^{-}$beam. Therefore, we used the SPEAM ${ }^{92}$ code to estimate this effect. It was impossible to reproduce the TRANSPORT calculations identically because SPEAM does not allow for extended fringe fields on the dipole magnet. We plotted the maximum beam size for the transverse planes through the transport system vs the effective beam current in Fig. 72 .

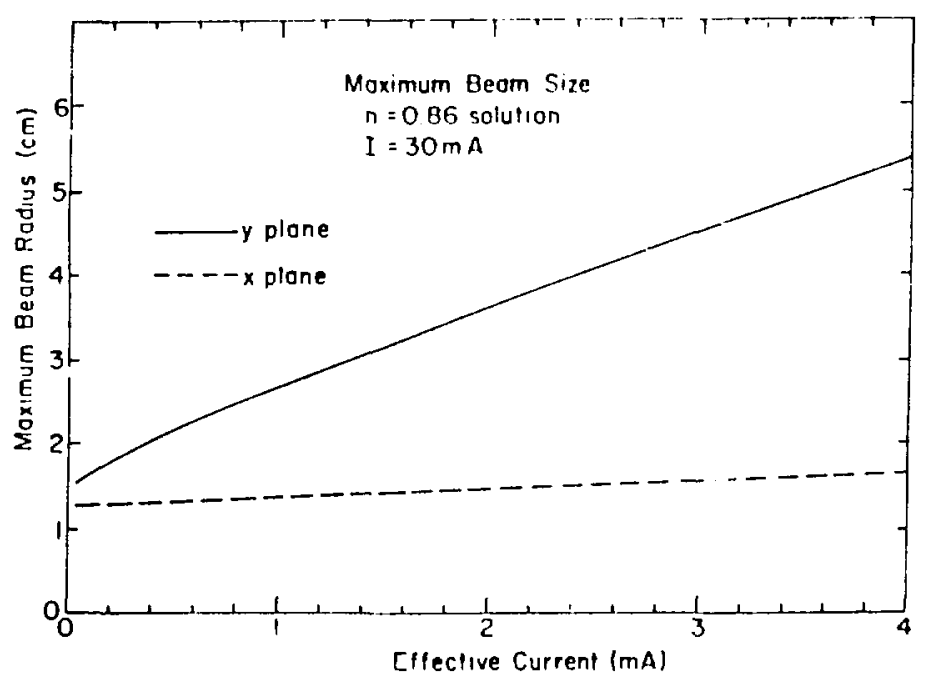

Fig. 72 .

Influence of an incompletely neutralized beam on the maximum beam radius. 
Calculations suggest triat the transverse $y$-plane growth may be a problem if the space-charge neutralization is less than $99 \%$.

F. Accelerating-Column Optics

1. First-Order Calculations. We studied beam optics for the accelerating column by using the beam envelope code SPEAM. ${ }^{10}$ The physical length of our accelerating region is $10 \mathrm{~cm}$. The initial phase space consists of a diverging beam in the $x$ plane and a converging beam in the $y$ plane with normalized emittances of 0.1 and $0.02 \pi \mathrm{cm} \cdot \mathrm{mrad}$, respectively. We conclude that an electrode aperture radius of $2-3 \mathrm{~cm}$ yields suitable beam properties for $\mathrm{H}^{-}$currents in the 10 - to 40-mA range.

2. Higher Order Calculations. Considerable effort has gone into the numerical evaluation ${ }^{93,94}$ of emittance growth during acceleration of intense particle beams. Our approach was to compare and use results from the third-order analytic program COLUMN ${ }^{95}$ and the trajectory-calculating program SNOW. ${ }^{94}$ In the program COLUMN, the envelope trajectories for a uniform density (but not necessarily round) beam with zero emittance were calculated using an analytic formula for the electric fields produced by accelerating electrodes with round apertures. The third-order corrections to the fields were added as a perturbation to calculate the phase-space distortion. SNOW is a self-consistent, cylindrically symmetric solution of Poisson's equation, and allows nonuniform, finite emittance beams.

The first three lines of Table XII show the SNOW and the COLUMN calculations of the effective emittance growth for a 200-keV, 100-mA beam.

The effective emittance is calculated from the area of a parallelogram that encloses the distorted zero-emittance curve in phase space. Injected beams that were parallel, diverging, and converging with a $1.0-\mathrm{cm}$ radius were studied. SNOW predicts an output beam radius $30 \%$ less than COLUMN. SNOW predicts emittance growths of less than $0.02 \pi \mathrm{cm} \cdot \mathrm{mrad}$ for the three uniform beam cases. The COLUMN results for emittance growth show the same trend.

II. ACCELERATOR TEST STAND

A. Injector

1. Overvien. We are designing the injector (Table XIII) for $\mathrm{H}^{-}$ current pulses of up to $100 \mathrm{~mA}$ at an energy of $250 \mathrm{keV}$ with duty factor of $10 \mathrm{~Hz}$ by $1 \mathrm{~ms}$. We are upgrading our Dudnikov-type source to provide the $\mathrm{H}^{-}$ ions. We will extract the beam at $20 \mathrm{kV}$, analyze and focus it with a $90^{\circ}$ 
TABLE XII

THEORETICAL PREDICTIONS ${ }^{a}$

$\begin{array}{r}(\mathrm{mrad} / \mathrm{cm}) \\ \text { Beam Input } \\ \hline\end{array}$

\section{Beam Radius (cm)}

1.0

1.0

1.0

1.0

1.0
Beam Profile

Uniform

Uniform

Uniform

Gaussian ${ }^{b}$

Gaussian ${ }^{b}$ $(\mathrm{mrad} / \mathrm{cm})$

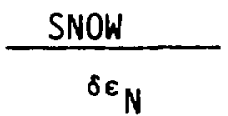

$\frac{(\pi \mathrm{cm} \cdot \mathrm{mrad})}{3 \times 10^{-2}}$

$1.3 \times 10^{-2}$

$1.6 \times 10^{-2}$

$1.3 \times 10^{-2}$

$3.8 \times 10^{-2}$

$3.8 \times 10^{-2}$ $(\mathrm{mrad} / \mathrm{cm})$

$\frac{\text { COLUMN }}{\delta \varepsilon_{N}}$

$\frac{1 \pi \mathrm{cm} \cdot \mathrm{mrad}}{2.1 \times 10^{-2}}$

$4.6 \times 10^{-2}$

$0.9 \times 10^{-2}$

aContains a comparison between the SNOW and COLUMN calculations for emittance growth of a 200-keV, $100 \mathrm{~mA}$, uniform-density beam. The last two lines show the effect of including the nonuniform beam profile in the SNOW calculations.

$\mathrm{b}_{\rho} / \rho_{0}=1 / \mathrm{e}$ at $\mathrm{R}=1.0 \mathrm{~cm}$.

magnet, then inject it into the 230-kV accelerating column after final focusing with a quadrupole doublet (Fig. 73). An isolated terminal at the column potential contains a rack for the magnet controls and two racks for the ion source, further isolated by the $20-k V$ extraction potentials. A $3000-l / s$ cryopump mounted near the ion source can give $100 \mathrm{~h}$ of continuous operation at the designed gas flow. A $2000-l / s$ diffusion pump mounted at ground potential will handle the high gas flow used when the source is started cold. Accelerating column sparking should be minimal because of the (1) all metal-ceramic design, (2) moderate values of beam voltage and current, and (3) magnetic analysis of the beam before injection into the column.

\section{TABLE XIII}

\section{LINAC INJECTOR SPECIFICATIONS}

Energy regulation with a series tube to $1 / 10^{3}$ $250 \mathrm{keV}$

Peak current of $\mathrm{H}^{-}$ions $100 \mathrm{~mA}$

Duty fictor- $10 \mathrm{~Hz}$ by $1 \mathrm{~ms}$ 

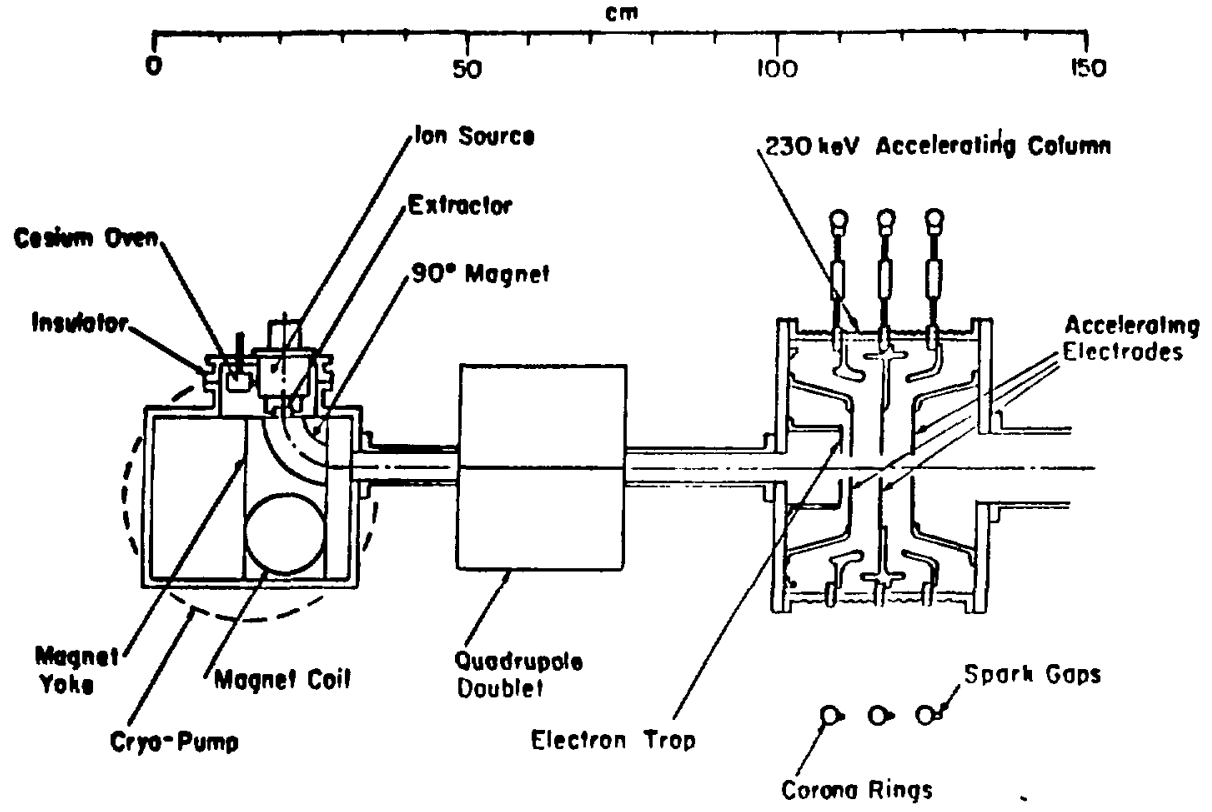

Fig. 73 .

Layout of $\mathrm{H}^{-}$injector for accelerator test stand.

The terminal will enclose the three power-supply racks, 20-kV isolation transformer, regulators, and the ion source, magnet, cryopump, and quadrupoles up to the accelerating column. The terminal's corners and edges have radij of $10 \mathrm{~cm}$, and the terminal is supported by six 100-cm-long PVC legs.

2. Source Design. We will modify our Dudnikov-source to provide

(a) improved cathode-anode alignment,

(b) source-extractor alignment, adjustable under operation,

(c) the source body as the vacuum-air interface to simplify feedthrough design, and

(d) independent cesium feed in addition to the internal feed.

The source will be isolated from the magnet box with a glass insulator and will slide on the mounting plate to provide alignment adjustments of about $1 / 4 \mathrm{~mm}$. Optics calculations show that a field index $n$ of about 1 is optimum for a 100-mA beam, and the poles can be changed to provide $n$ values appropriate to other conditions. The magnet box pressure is about $10^{-5}$ torr at the design gas flow. 


\section{Accelerating Column}

a. Ceramics Assembly. Our accelerating tube assembly has a four-section metal-ceramic braze structure patterned very closely after the column constructed for the Lawrence Livermore Laboratory's RTNS accel-

erator. ${ }^{96}$ Each ceramic is $6.7 \mathrm{~cm}$ long, yielding an electric field of $8.6 \mathrm{kV} / \mathrm{cm}$ at $230 \mathrm{kV}$ on the tube. A gradient of $6.0 \mathrm{kV} / \mathrm{cm}$ is used on the RTNS project, which runs at $1 \mathrm{~atm}$ of air without flashover; however, because of our greater field and higher altitude, it seems likely that we will have to run in an $\mathrm{SF}_{6}$ atmosphere.

b. Electrode Design. The accelerating electrodes, their supports, and $x$-ray shields are designed so that the maximum electrical stress is less than $100 \mathrm{kV} / \mathrm{cm}{ }^{97}$--well below the vacuum breakdown region of Kilpatrick. ${ }^{98}$ Figure 73 shows the electrode structure. The first electrode is an electron trap (decel), and the succeeding electrodes are at $0-, 115-$, and 230-kV potential.

We have all parts for both the $230-k V$ and the $20-k V$ regulators and will use the 300-kV, 250-mA power supply, and the 300-kV, 10-kVA power isolation transformer that was bought and delivered for the high-current test stand. The 30-kV, 5-mA supply and the 30-kV, 5-kVA transformer for the ion source have been ordered, and they are short-delivery-time items.

B. Beam Transport Between the Buncher and Accelerator

The accelerator test stand, discussed in Sec. II.A above, will use a single, two-frequency buncher. Because of the high frequency and low energy of the injection system, there is not room for a conventional two-doublet matching system between the buncher and accelerator, if the doublets are electromagnets. The two possibilities are doublets made of variable permanent quadrupoles or extending the periodic structure of the accelerator up to the buncher. The latter system is the simplest optically and has the added advantage of maintaining the smallest possible beam in both the buncher and the quadrupoles, thus minimizing emittance growth that is due to aberritions. We chose a periodic structure that had the same transverse-phase shift as the first accelerator cell. Accordingly, the quadrupoles' gradients are somewhat weaker bezause there is no rf defocusing. 
There are several possible sources of emittance growth in the buncher-transport system. The buncher itself is a nonlinear defocusins element. Its effect is minimized if the beam is small at the buncher. The other sources of emittance growth are aberrations in the quadrupole system. These are of three types: chromatic aberrations caused by the momentum spread intruduced by the bunciner; third, and higher-order geometric aberrations; and harmonics caused by the quadrupole construction. Space charge aggravates all of these effects. Chromatic aberration is potentially the most damaging of these effects because it is a second-order effect. The ray-tracing program, TURTLE, was used to explore the magnitude of these aberrations in the absence of space charge. We traced 5000 rays that were uniformly distributed within the phase ellipse, with a momentum spread of $\pm 2.2 \%$ (also uniformly distributed) corresponding to an $11-k V$ peak voltage on the buncher. The emittance growth to the center of the first accelerator quad was negiigibie. With a momentum spread of $\pm 5 \%$ corresponding to a burcher voltage of $25 \mathrm{keV}$, approximately $2 \%$ of the particles fell outside the monochromatic spot. We a) investigated beams mismatched in size by $25 \%$.

In none of these cases does the emittance growth exceed a few per cent. A second-order TRANSPORT matrix calculation that assumes a Gaussian beam, gives an emittance growth of $4 \%$. To check these results, we ran the same system with PARMILA, including the rf effects from the buncher. We saw no emittance growth at all with 500 particles and low current.

We conclude that for a reasonably well-matched beam, chromatic aberrations in the transport following the buncher are small in the absence of space charge.

C. Laser Diagnostic Development

The measurement of emittance growth at high-current densities requires the use of neutral particle diagnostics. We plan to use a Nd: IAG picosecond laser to photodetach the extra electron from the $\mathrm{H}^{-}$ion. The neutral particles then simply drift to the detection plane. Apart from the use of neutral particles to avoid space-charge difficulties, the diagnostic is equivalent to conventional emittance-measuring devices, with the laser beam replacing the slit for limiting the initial beam width.

The laser produces $10-\mathrm{mJ}$ pulses at a $10-\mathrm{Hz}$ repetition rate. A fastpulse slicer selects a single pulse per shot from the mode-locked train from 
the oscillator. For diagnostic use, the laser pulse must produce a sufficiently high contrast to distinguish the neutrals produced by photodetachment from neutrals produced by collisions with background gas in the accelerator. Also, the laser pulse must be correlated to the rf phase. We have built equipment to experimentally address both issues and have completed calculations that indicate solutions to both problems are available.

For the neutral-background measurements, we will use a laser source to photodetach the electrons from the $\mathrm{H}^{-}$beam at the Los Alamos Vertical Van de Graaff Facility and to determine the pressure dependence of the contrast ratio. The correlation to rf phase will be by deflection or retardation of a lasergenerated electron beam or a time-to-amplitlide converter with 5-ps or less jitter. We are experimentally measuring the laser pulse signal size to select the final method for achieving precision of 50 ps or better.

We have considered schemes to time the laser diagnostic pulse to the $\mathrm{rf}$ phase of the accelerator. The need for this timing arises because the generation of 30-ps laser-pulses requires use of saturable dye for mode locking, resulting in a random start time for the pulse train on a submicroscecond time scals.

The assumptions used to evaluate the scheme are that the operating frequency of the linac is $440 \mathrm{MHz}$ (2.27-ns period) and that the phase interval containing beam particles is $\$ 30^{\circ}$, or an equivalent time interval of $0.38 \mathrm{~ns}$. A determination of the time to 50 ps then represents bins of approximately one-eighth of the beam pulse length for examining longitudinal behavior of the beam bunch.

The most straightforward means to detect time intervals of this magnitude is to examine the output of a fast photodiode with an oscilloscope. Figure 74 shows the measurement condition and proposed setup. For this configuration, the oscilloscope should have required display resolution. The frequency response of the oscilloscope is another matter, however, and it is not clear that this method can give reliable detection on a 50-ps time scale.

Because of the difficulties in observing the time history of the pulse directly, we considered schemes in which the time history of the light pulse is converted to a spatial position. 


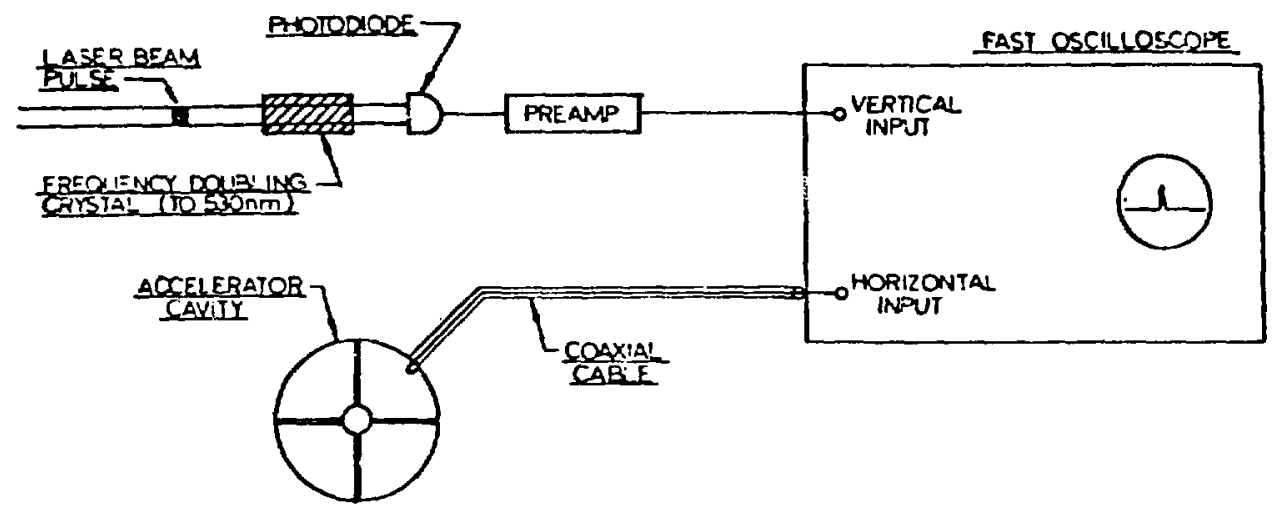

Fig. 74.

Schematic diagram of system to correlate laser pulse to $\mathrm{rf}$ phase using direct detection of the laser light in a photodiode.

Figure 75 illustrates the basic principle. The rf signal from the linac cavity is tapped at two points of a terminated transmission line. This produces a circular Lissajous figure in the detector plane if the points for the taps are chosen appropriately. At the time the laser fires, a detector pulse is produced by the light striking a photocathode.
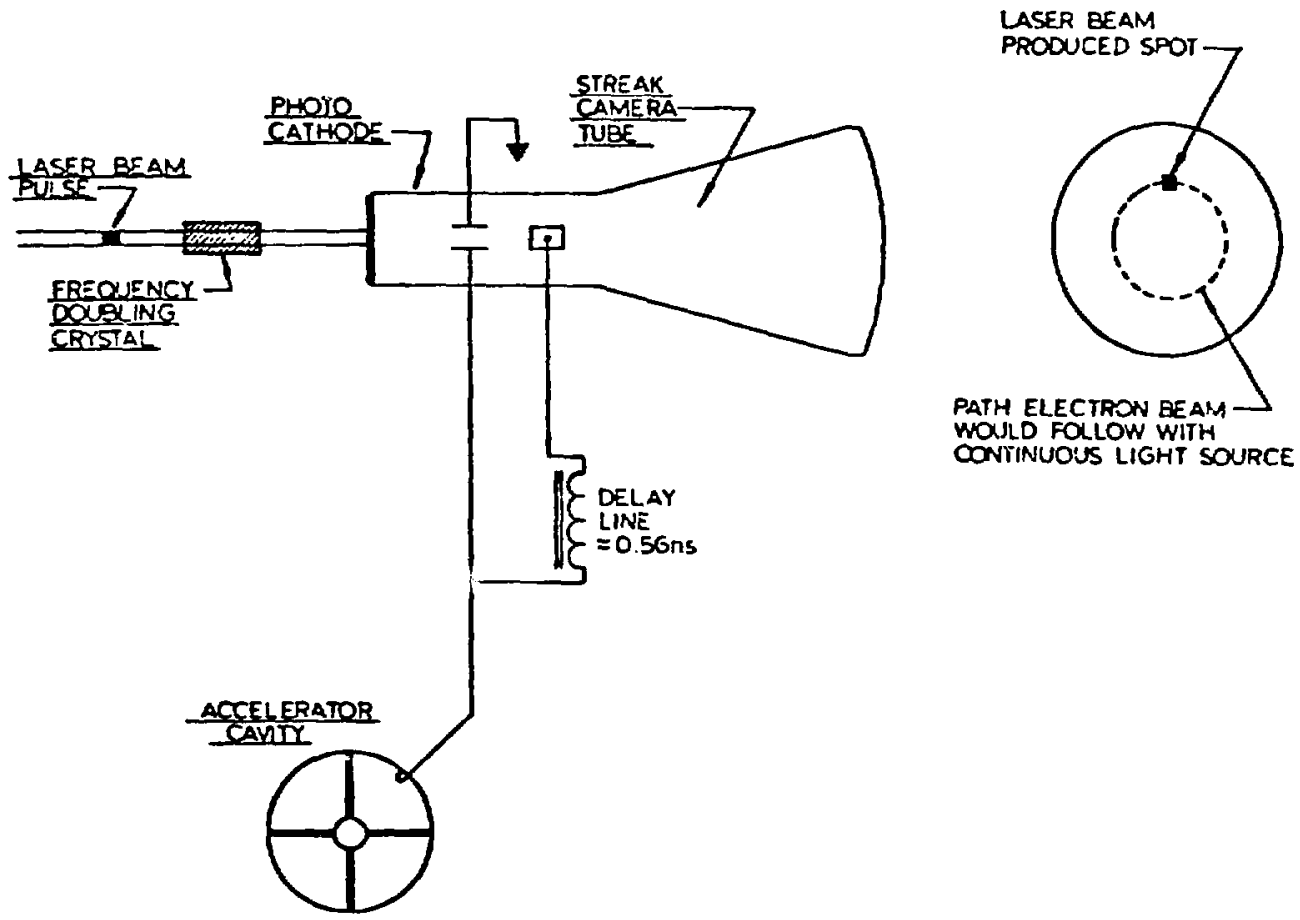

Fig. 75.

Schematic diagram of system to correlate laser pulse to rf phase using Lissajous figure and streak camera. 
Provided signal amplitude can be determined, unambiguous phase information can be obtained in one dimension. Two light beams with a definite time delay are produced by passing the laser beam through an etalon that transmits about 50 to $60 \%$ of the light. The second pulse has an amplitude $1 / 4$ to $1 / 6$ that of the first and occurs later in time. Figures 76 and 77 illustrate application to a scheme in which the radio frequency is used to accelerate and decelerate the electron beam and energy analysis is made to determine the phase.

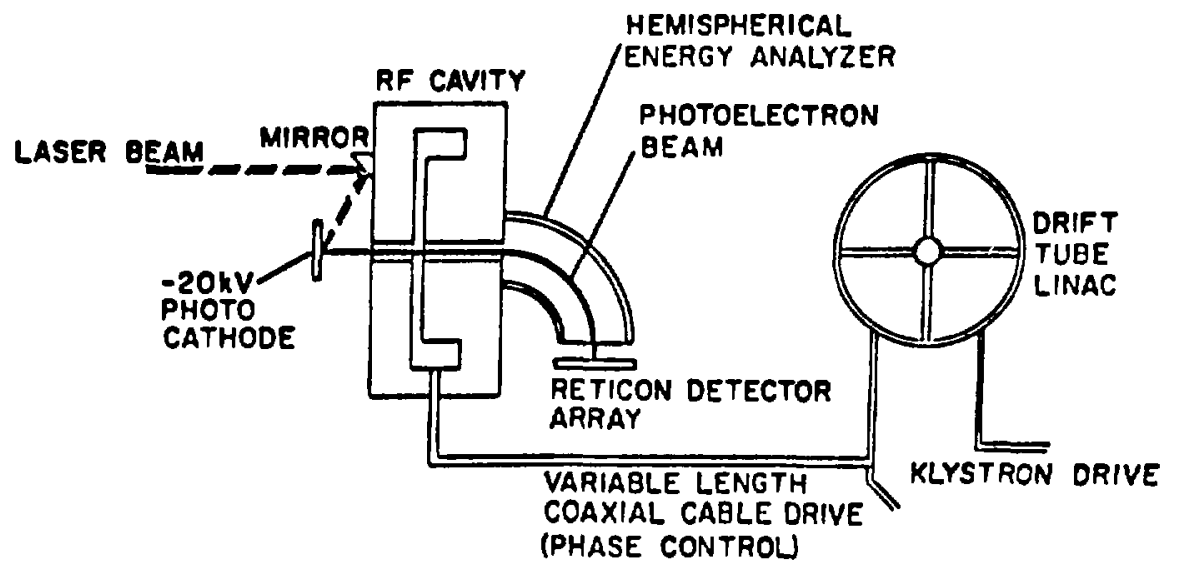

Fig. 76.

Schematic diagram of system to correlate laser pulse to $r f$ signal using modulation of electron beam energy. Two beams of known time delay can be generated by inserting a pair of mirrors (or Fabry-Perot etalon) in the laser beam.

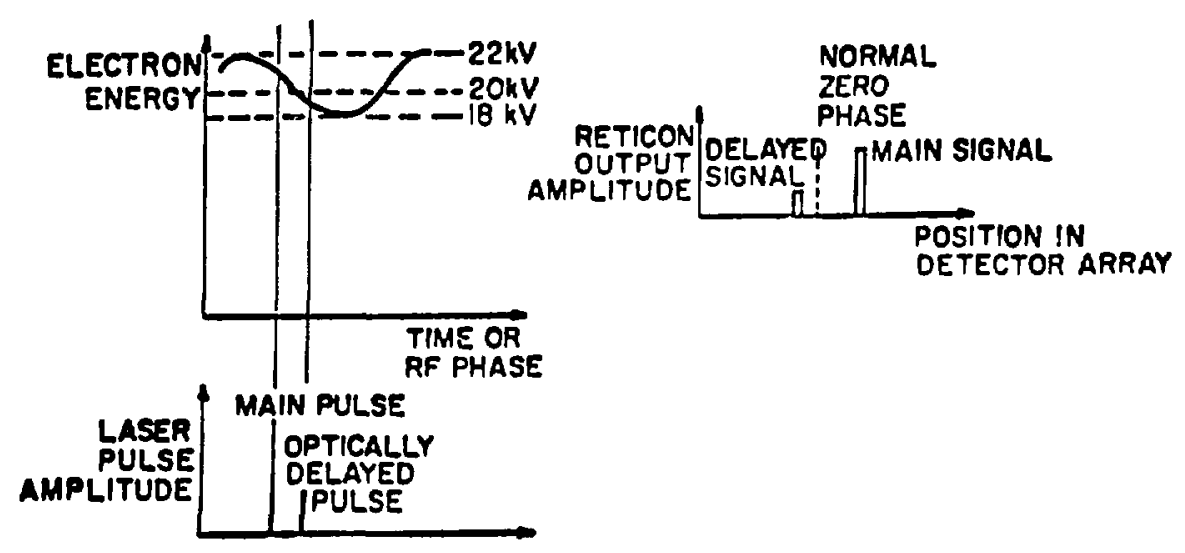

Fig. 77.

Schematic diagram of laser and electricai signals for the apparatus illustrated in Fig. 76. The combination of energy position and signal amplitude defines the rf phase at which the laser pulse occurred unambiguously. 
D. Three-Cell Test-Stand Linac Cavity

We have designed a three-cell lirac cavity for test stand use to determine the manner in which a beam of particles with known attributes is transported through the cavity. We will measure beam properties before reaching, in the center of drift tubes, and after leaving, the linac. We will compare these measurements with predictions made with existing computer simulation programs (mainly PARMILA) to determine the validity of the predictions. If discrepancies occur, we expect to determine the cause and make the appropriate computer-code modifications.

This cavity design was limited by several constraints: injection energy of $250 \mathrm{keV}$, maximum quadrupole gradient of $25 \mathrm{~kg} / \mathrm{cm}$, and maximum electric field of $30 \mathrm{MV} / \mathrm{m}$. The design had to be compatible with the laser diagnostics and we wished to maximize the total current that could be stably transported.

The cavity parameters are cell length $2 \beta \lambda$, injection energy $250 \mathrm{keV}$, final energy $318.9 \mathrm{keV}$, average electric field $2 \mathrm{MV} / \mathrm{m}$, maximum electric field $30 \mathrm{MV} / \mathrm{m}$, frequency $440 \mathrm{MHz}$, transit-time factors $(T=0.46, S=0.57)$, synchronous phase $-45^{\circ}$, and total length $9.7 \mathrm{~cm}$. We gave no consideration to maximizing $Q$ or shunt impedance $Z$. The maximum current that $c$ an be transported, both longitudinally and vertically, is $17.5 \mathrm{~mA}$. The beam radius for $17.5 \mathrm{~mA}$ is half the bore radius, which is large enough so that particles neutralized in the cavity by the diagnostic laser can drift out of the linac without striking the bore tube. The gaps between drift tubes were minimized, mainly to maximize the lengths of the quadrupole magnets in the drift tubes, and also to maximize the transit-time factor. The permanent magnet material quadrupoles are a new design. The $25-\mathrm{kG} / \mathrm{cm}$ quadrupole field gradient is limited by the maximum pole-tip field and bore size. The $2 \beta \lambda$ cell length was chosen over $1_{B \lambda}$ to increase transverse focusing.

The best available material for the permanent magnet quadrupoles is Hicorex 90B, which we have chosen for the accelerator test-stand quadrupoles. For this material, the pole-tip field is limited to approximately $1.25 \mathrm{~T}$. We chose an aperture radius of $5 \mathrm{~mm}$ to allow some room for investigating mismatched and off-center beams. 
I. BUNCHER STUDIES

Multiple Harmonic Buncher. Longitudinal matching into a linear accelerator of an initial dc beam is generally accomplished by the use of a buncher. The simplest buncher is one rf cavity tuned to resonate at the linac frequency. This buncher will place $\sim 60 \%$ of the initial dc beam into the longitudinal phase acceptance of the accelerator. In principle, given the correct waveform in the buncher, one could place the entire bunched beam into the accelerator acceptance. If we could design a cavity to resonate at all harmonic frequencies, as well as the fundamental, we could approach any desired waveform for a buncher. We would like to determine if several cavities, each resonating at a single frequency, can achieve the same result as a single buncher cavity that resonates at several frequencies. We expect that the two methods of bunching will be equivalent if the several, single harmonic burichers all act within a time period that is short, compared to the time for bunching.

Our multiple-cavity buncher requires that the phases of the individual particles in the beam change little, with respect to the synchronous particle, while they are passing through the set of bunchers. In a converging harmonic series, the coefficients for the Fourier components get smailer as the harmonic increases. We determined the effect of a buncher in which the highest harmonic cavities (smallest voltage amplitude) appeared to the beam first, followed by cavities with lower harmonic value, and ending with the fundamental frequency.

We considered a series of pill-box cavities operating in the $\mathrm{TM}_{010^{-l i k e}}$ mode (Fig. 78) and compared this with a single multiple-frequency buncher. The single-cavity buncher resonated at 10 frequencies, with the Fourier coefficients matched to a straight-line waveform of energy vs phase. The maximum applied voltage was $50 \mathrm{kV}$, which would bunch a $250-\mathrm{KeV}$ beam in $8.6 \mathrm{~cm}$. The multiple cavities had their amplitudes set to the same value as the corresponding frequency amplitude in the single, multiple-frequency buncher. The phases of the cavities were set such that the synchronous particle received no impulse. The separation between the cavity centers was $0.5 \mathrm{~cm}$, and the cavities were ordered such that the highest frequencies occurred first (case 1). We also considered the geometry in which the order of the cavity frequencies was reversed (case 2). Figure 79 shows the results. The poor fit near $180^{\circ}$ 


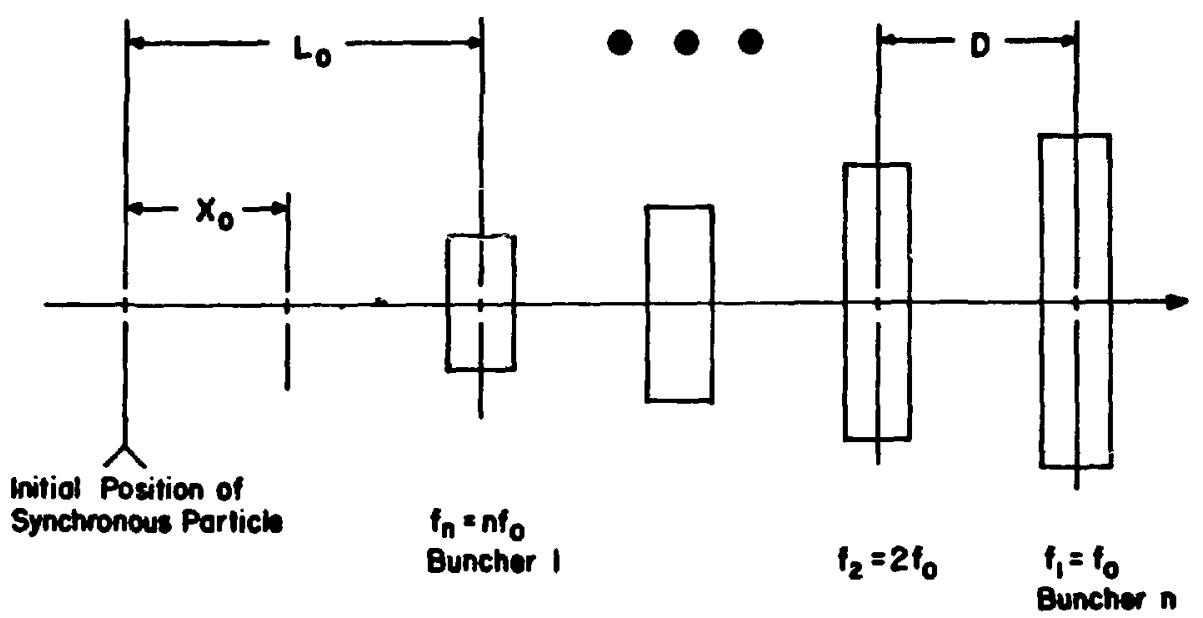

Fig. 78.

Geometry for the multiple cavity buncher calculations. The highest frequency cavity is shown to occur first followed by lower frequency cavities. $\underline{D}$ is the separation between cavitites.
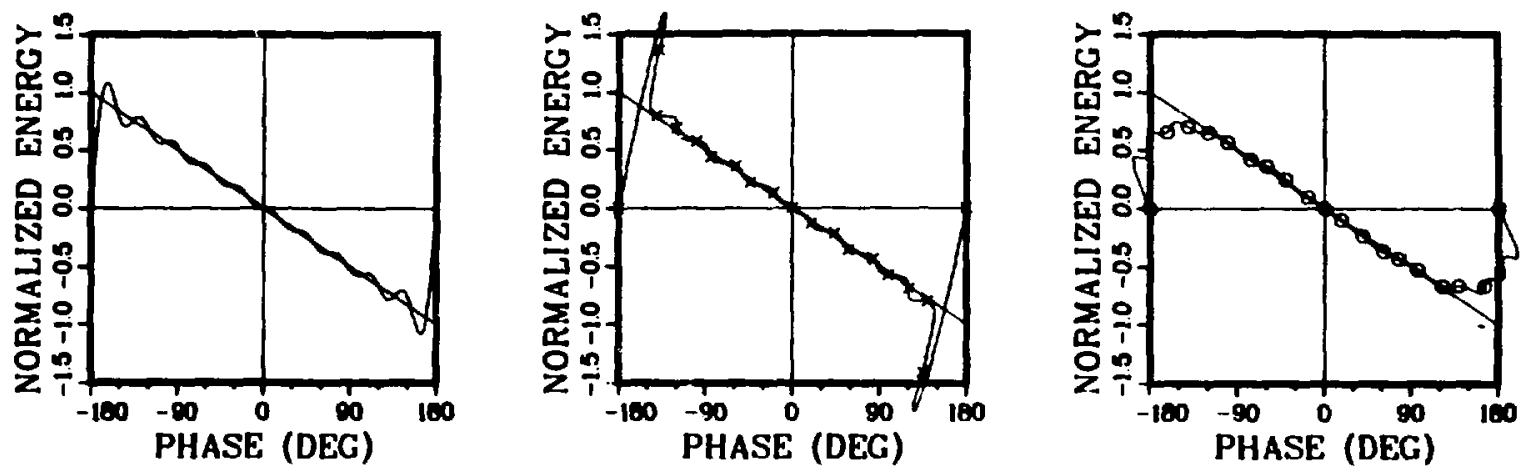

Fig. 79 .

Results for a 10-frequency buncher system with all the harmonic amplitudes held fixed. The figure on the left shows a Fourier fit to a straight line (i.e., all harmonics are generated in a single cavity). The center and right figures show the results where the individual frequencies occur in separate cavities. The center figure corresponds to high frequencies first and the right figure to low frequencies first. 
for case 1 is because all the Fourier amplitudes near $180^{\circ}$ have the same sign, and decrease to zero at $180^{\circ}$. The effect of each cavity is to decrease the magnitude of the phase of a particle such that it sees a larger amplitude than it should in each succeeding cavity. At first it seems remarkable that this series of cavities, separated by a total distance of $4.5 \mathrm{~cm}$ compared to the 8.6-cm bunching distance, reproduces the effect of the single-cavity buncher so well. We can show, that for a linear waveform and case 1 , the total phase change of a particle (traveling between the nth harmonic cavity and the fundamental frequency cavity) that is due to the nth harmonic cavity, is on average $(\pi D / L)$, where $D$ is the spacing between cavities and $L$ is the length for bunching. This relation is independent of $N$. We are developing a computer program, BUNCHER, that will optimize the voltage amplitude of a series of cavities to produce a desired distribution of particle energy vs phase.

This program determines the operating parameters for a buncher system consisting of several cavities, each of which resonates at some multiple of the fundamental linac frequency. Only longitudinal phase space is considered. We also assume that appreciable bunching does not occur in the time the beam passes through the buncher system, so that space-charge forces may be ignored. We determine the buncher parameters by requiring that the longitudinal phasespace distribution at the buncher's downstream end match the phase-space distribution determined by drifting a "matched" bean from the linac entrance back to the buncher's downstream end. To test the program, we assume that the required phase-space distribution is linear in energy vs phase. We use a 30-cm bunching length (distance between the final, downstream, buncher cavity and the linac); a $2.5-\mathrm{cm}$ distance between each cavity (D in Fig. 78); a $440 \times 10^{6} \mathrm{~Hz}$ linac frequency; and a $250-\mathrm{keV}$ proton energy. We vary the number of cavities from 1 to 9 , with the highest frequency cavity first. The maximum, effective, buncher voltage is $12.6 \mathrm{kV}$ (corresponding to $180^{\circ}$ of phase in the linear ramp). This will bunch a dc beam ( $\pm 180^{\circ}$ phase spread) down to $\pm 0^{\circ}$ phase spread in $30 \mathrm{~cm}$ in the absence of space charge. Table XIV lists the results. Figure 80 shows the phase-space distribution that ideally should follow the linear ramp. With this model, we expect 92 capture with four cavities (perhaps 2 double-harmonic cavities). 
TABLE XIV

RESULTS OF BUNCHER CALCULATIONS

Number

of

Cavities If $\quad 2 f \quad 3 f \quad 4 f \quad 5 f \quad 6 f \quad 7 f \quad 8 f \quad 9 f \quad$ (Relative)

\begin{tabular}{|c|c|c|c|c|c|c|c|c|}
\hline 8.03 & & & & & & & & \\
\hline 7.83 & -3.87 & & & & & & & \\
\hline 7.69 & -3.44 & 2.35 & & & & & & \\
\hline 7.60 & -3.36 & 1.84 & -1.71 & & & & & \\
\hline 7.56 & -3.28 & 1.72 & -1.21 & 1.26 & & & & \\
\hline 7.54 & -3.23 & 1.71 & -1.08 & 0.79 & -1.01 & & & \\
\hline 7.53 & -3.20 & 1.67 & -1.06 & 0.66 & -0.58 & 0.81 & & \\
\hline 7.52 & -3.18 & 1.63 & -1.07 & 0.64 & -0.46 & 0.42 & -0.68 & \\
\hline 7.52 & -3.17 & 1.62 & -1.04 & 0.65 & -0.44 & 0.31 & -0.34 & 0.58 \\
\hline
\end{tabular}
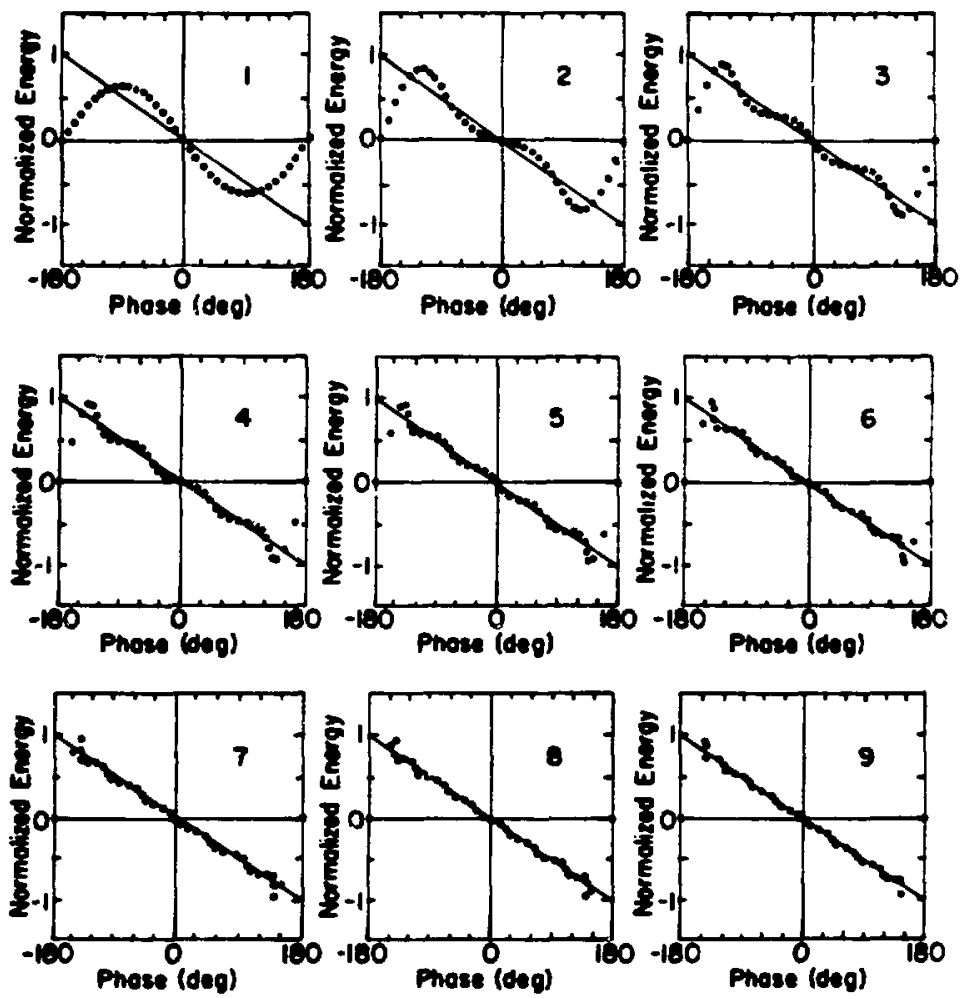

Fig. 80 .

Longitudinal phase space distributions from multiple cavity (and harmonic) buncher systems. The number in the upper right of each figure corresponds to the number of separate cavities and frequencies. 
II. LINAC-BEAM DYNAMICS

A. Introduction

We continued our study of linear accelerator beam dyramics. Our emphas is was on studying space-charge effects (or other effects in a spacecharge limited beam) in a simple model without the complications of many accelerator details. We looked at an azimuthally symmetric bunched beam in an acccelerator in which the focusing and accelerating forces on the bunch are time-independent (smooth approximation) or vary slowly (negligible variation over an rf or focusing cell). An important part of our work was the determination of phase-space distributions matched to a given accelerator. We define these matched or equilibrium distributions as time-independent distributions for an accelerator with focusing forces having no explicit time dependence. With space charge, we must consider the self forces in addition to the external focusing forces. A matched distribution injected into an accelerator, with parameters slowly varying in time, remains instantaneously at equilibrium; that is, it remains time-independent if the accelerator parameters are held constant after a given instant. For periodic or nearly periodic focusing systems, we generalize that a matched distribution is periodic with the same period as that of the focusing structure. We have constructed azimuthally symetric phase-space distributions for a bunched beam, matched to an accelerator with constant, but nonlinear, az imuthally symetric restoring forces. Others have used either the K-V distribution or the uniformly charged ellipsoid in previous work done in this field. The K-V distribution has uniform charge density and is valid only for continuous (not bunched) beams. It is useful because its $\cdot 1$ inearity makes certain calculations possible and because it is approximately matched, even for a periodic-focusing system. There is no analog of the $K-V$ distribution for bunched beams. Others often use uniformly charged ellipsoids; but these are not equilibrium distributions, even for constant focusing forces, so that these distribution calculations are not self-consistent. Creation of a matched distribution is useful for the following reasons. 
1. Accelerator Acceptance. With no space charge, a matched twodimensional phase-space distribution has the same shape as that of the outermost particles trajectories. In a matched distribution no trajectories enclose empty regions of phase space. The accelerator bore or longitudinal potential well size determines a certain, limiting, phase-space trajectory. The area enclosed by this limiting trajectory is the acceptance or the maximum transmitted emittance. With space charge, there is no unique acceptance. Consider a matched distribution with a certain emittance corresponding to a very low current for which space-charge forces are negligible. Now raise the current, keeping the emittance value and all accelerator parameters fixed. For lowcurrent values, we simply can increase the phase-space density without otherwise changing the distribution and maintain a match. Once space-charge forces become significani however, the distribution must increase in spatial extent to remain matched because the reduced net restoring force means a particle must depart farther from the axis than before, to convert the given kinetic energy into potential energy. The current $c$ an be raised indefinitely, maintaining a match for a given emittance and accelerator except for limitation related to the increasing beam radius and bunch length.

2. Scaling Laws. The emittance in any direction, the current, or any other integrated property of the 60 phase-space distribution function $c$ an be written as a function of accelerator parameters multiplied by a nonunique constant, which depeno's on the equilibrium-distribution type. We find such a relation useful in determining the best parameter values for a given requirement on current, emittance, or other property.

3. Matched Beam Parameters. An equilibrium distribution corresponding to the initial accelerator structure gives the beam properties required at injection. These are called the matched beam parameters.

4. Check on Particle Tracing Codes. An important use of equilibrium distributions is in checking particle tracing codes for numerical problems. These distributions $c$ an serve as the initial conditions for the system solved by the tracing code. Because we know that the code should keep the distribution constant with time, we can measure directly the important collision enchancement effect caused by the limited number of macroparticles because any observed emittance growth for one of these distributions will be purely numerical; similarly, we can determine integrator step-size requirements. This 
procedure is much safer than trying to determine a regime independent of numerical parameters on a general initial-condition problem because some numerical errors may depend very slowly on numerical parameters.

We have written a new version of the $r-z$ equilibrium-distribution generating code, named RZED79. The main improvement over the previous RZED code is that we take into account the axial nonlinearity and the nonlinear $r f$ coupling forces. We moditied the $r-z$ particle-in-cell tracing code HOT to include these nonlinear focusing forces and made some progress in producing a three-dimensional space-charge routine for the HOT code.

B. Linear Smooth-Focusing and Discrete-Acce lerating Gaps

Here we summarize results that were reported at the 1979 Particle Accelerator Conference. 99 We tried different initial distributions for a fixed accelerator, with continuous linear external-focusing and continuous acceleration. As expected, we saw no change for a matched distribution as we accelerated the beam. We saw little emittance growth when we reduced the beam radius to below its matched value, keeping the emittance and current fixed, but the beam radius grew to above its matched value. For example, if a beam of one-half the matched radius is injected into the accelerator, the radius will grow by more than a factor of two.

We studied localizing the axial forces to the gap locations with a special version of HOT, to compare with the smooth approximation usually used. We accelerated the beam across 150 gaps and found the same degree of phase damping still present.

C. Different $x$ and $y$ Emittances

We produced a distribution with an emittance ratio of ten between the $x$ and $y$ planes by deforming a $\mu=0.8$ matched distribution, $\mu$ is the ratio of the space-charge force to the applied external force. (The result was not a matched distribution. We do not have the means yet to produce matched distributions without azimuthal symmetry.) This beam was accelerated from $0.25 \mathrm{MeV}$ to $1.0 \mathrm{MeV}$ in an accelerator with constant transverse phase advances and a constant electric field.

The initial and final normalized emittances, containing $70 \%$ of the particles, are shown in Table XV. 
TABLE XV

EMITTANCES FOR $\varepsilon_{x} / \varepsilon_{y}=10 \pi$ INITIAL BEAM
(in $\mathrm{cm} \cdot \mathrm{mr}$ ad)

$$
\varepsilon_{x} / \pi \quad \varepsilon_{y} / \pi \quad \varepsilon_{x}+\varepsilon_{y}{ }^{+} \varepsilon_{z}
$$

$\begin{array}{llll}\text { Initial } & 0.230 & 0.022 & 0.3243 \\ \text { Final } & 0.176 & 0.0611 & 0.3278 \\ \text { Final/initial } & 0.077 & 0.269 & 0.101\end{array}$

The $x$ and $y$ emittance values approach each other as the beam is accelerated and the sum of the three emittances is conserved to high accuracy. D. Phase-Space Distributions Matched to External Nonlinearities

1. The RZED79 Code. Previously, we numerically calculated equilibrium distributions in $r-z$ cordinates by the code RZED. We used the results to produce the numerical constant for the scaling laws and as initial distributions in particle-tracing calculations. We have written a new version called RZED79, with the following differences.

a. Nonlinear External Forces. The main reason we developed RZED79 was to include nonlinear and coupling terms in the external focusing forces. Our previous studies with purely harmonic external focusing, used nonlinearities and couplings corning only from space charge; they did not show any significant emittance growth. To effectively do particle-tracing simulations with nonlinear external focusing forces we needed to have phase-space distributions matched to the forces. We added the terms $z^{3}$ (axial nonlinearity) and $r^{2} z$ (rf coupling) in the external Hamiltonian to the harmonic focusing potential that we used previously. In the normalized variables of Ref. 100 , the added terms are evident in the following expression

$\frac{H_{0}-V}{H_{0}-e \phi_{0}}=1+\Phi_{0}-\Phi-\frac{R^{2}+a Z^{2}}{2(2+a)}+\frac{\operatorname{anscot} \phi_{s} Z^{3}}{3(2+\alpha)}-\frac{\operatorname{ar\delta cot} \phi_{s} R^{2} Z}{2(2+\alpha)}$. 
The two new parameters are the synchronous phase and the nonlinearity strength $\delta$. The meaning of $\delta$ is given by

$\frac{r_{\max }}{\beta \lambda}=\delta R_{\max }$

in which $R_{\max }$ is the maximum of the normalized radius variable $R$. The parameter $\delta$ measures how far the axial potential well is filled. For nonzero $\delta$, the scaling laws are more restricted. Once the RZED79 parameters $\mu, a, N$, $\phi_{\mathrm{S}}$, $\delta$ and the accelerator parameters are specified, the equilibrium distribution is completely specified.

b. Cylindrical Conducting Boundary. The far-away boundary that we produced by the stretched mesh of the RZED code has been replaced by a conducting cylinder near the particle bunch but we have not investigated the wall effect. (Particle-tracing simulations with initial distributions matched to a nearby conducting wall, traced through an accelerator with no conducting walls, showed that equilibrium is maintained to within the statistical accuracy). Probably the only important wall effect occurs for off-axis beams but we must study this with a three-dimensional calculation.

c. Space-Charge Potential Fit. The output of RZED79 is the space-charge potential, which is specified by the coefficients in a rational approximation (fourth-order numerator, fifth-order denominator, 34-coefficients total). This replaces the previous fit to a polynomial that was less accurate for high- $\mu$ distributions and did not al low for beams not symmetric about $z=0$.

2. HOT-Code Improvements. The HOT particle-tracing code, which uses an $r-z$ mesh, particle-in-cell, area-weighted, space-charge routine, has been modified to accept the new RZED79 output. Also, true phase-space areas non can be determined without fitting to ellipses. We obtain this output, which is useful for phase-space projections hat are not elliptical in shape, by dividing the phase-space region containing the particles in up to 30 pieces in each direction, and counting bins.

The initial distribution-generating routine in HOT uses the information from RZED79 to create a finite particle representation of the desired phase-space distribution by using up to 10000 macroparticles. We show the statistical errors in the emittances in Table XVI that we obtained by taking 
the standard deviations of ten runs, using different random sequences. The distribution in this example corresponds to large space charge and external nonlinearity effects $\left(\mu=0.8, \alpha=1, n=2, \phi_{s}=-46.4^{\circ}, \delta=0.05\right)$ using the maximum 10000 particles. The emittances are from the ellipse-fitting method.

We do not have the 3D, particle-tracing code ready. We have converted the HOT code to run on the CRAY-1 computer and have written a 3D, cylindrical, coordinate, Poisson solver and charge-weighting routine.

3. Distribution Properties With $z^{3}$ Terms. We use here, as an example, the ATS $2 \beta \lambda$ drift-tube linear accelerator. Table XVII shows the relevant parameters.

\section{TABLE XVI}

STATISTICAL ERRORS (IN PER CENT) IN EMITTANCE FOR INITIAL DISTRIBUTIONS (cm mrad)

90

of beam

of beam

rms

$\begin{array}{llll}\varepsilon_{x} & 3.4 & 1.3 & 1.0 \\ \varepsilon_{z} & 9.9 & 1.3 & 1.3 \\ \varepsilon_{x}+\varepsilon_{y} & 2.5 & 0.9 & 0.5 \\ \varepsilon_{x}+\varepsilon_{y}+\varepsilon_{z} & 4.9 & 0.7 & 0.5\end{array}$

TABLE XVII

ATS $2 \beta \lambda$ LINAC PARAMETERS.

Injection energy

Frequency

Quadrupole gradients

Quadrupole lengths

Accelerating field

Transit-time factor

Synchronous phase
$250 \mathrm{keV}$

$440 \mathrm{MHz}$

$230 \mathrm{~T} / \mathrm{m}$

$25 \mathrm{~mm}$

$2.05 \mathrm{MV} / \mathrm{m}$

0.5

$-46.4 \mathrm{deg}$ 
For these parameters, the zero-current phase advances per focusing period (length $4 B \lambda$ ) are $90^{\circ}$ in both the transverse and longitudinal directions.

We determined the accelerator's maximum current by looking at $\delta=0.8$, $\alpha=1, n=2$ distributions with a $z^{3}$ term (axial nonlinearity) but no $r f$ coupling term. We increased the parameter $\delta$ until the RZED79 failed to find an equilibrium distribution (we do not know whether this limitation was real or numerical). The corresponding current increased with $\delta$ up to the maximum value of 0.05 . The current at this $\delta$ value was $75.5 \mathrm{~mA}$ and the total normalized transverse emittance value was $\varepsilon=0.29 \pi \mathrm{cm} \cdot \mathrm{mrad}$. The beam radius was $\beta \lambda / 6.0=2.6 \mathrm{~mm}$ (probably too large to be practical but we are interested here mainly in learning properties in the regime where external nonlinearities are important). The potentials and charge density along the z-axis for this case are shown in Fig. 81. All quantities are in the normalized units of Ref. 100. The external potential is labeled $V_{E X T}$. This potential differs significantly from the harmonic potential because the $z^{3}$ term causes the potential well to have a finite width and height. The space-charge potential is labeled $\Phi$ and the sum of the external and space-charge potentials is

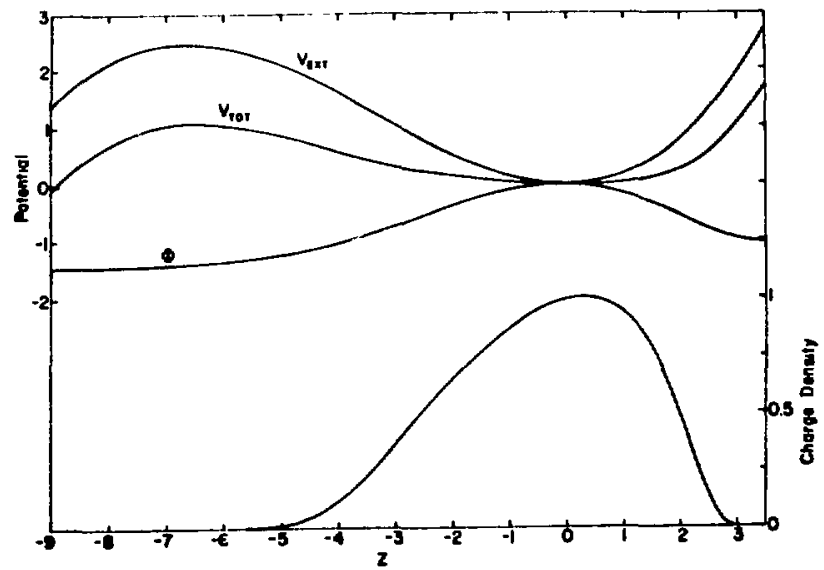

Fig. 81.

(1) The external, space charge, and total potentials along the $z$-axis are shown for a bunched beam matched to a smooth focusing linear accelerator containing the $z^{3}-a x i a l$ nonlinearity. The charge density a long the $z$-axis is shown in the bottom curve. $l$ abeled $V_{\text {TOT }}$. The interesting result is that the total potential well is almost as wide as the external one and that it is almost completely filled with particles. The reason for this behavior is the very nonuniform distribution of charge as seen in the bottom curve of Fig. 81 .

We obtained a series of different distributions, matched to the same accelerator, for lower values of the space-charge parameter $\mu$. For each $\mu$-value, we adjusted the value of $\delta$ to maintain the emittance at $0.29 \pi \mathrm{cm} \cdot \mathrm{mrad}$. We show the 


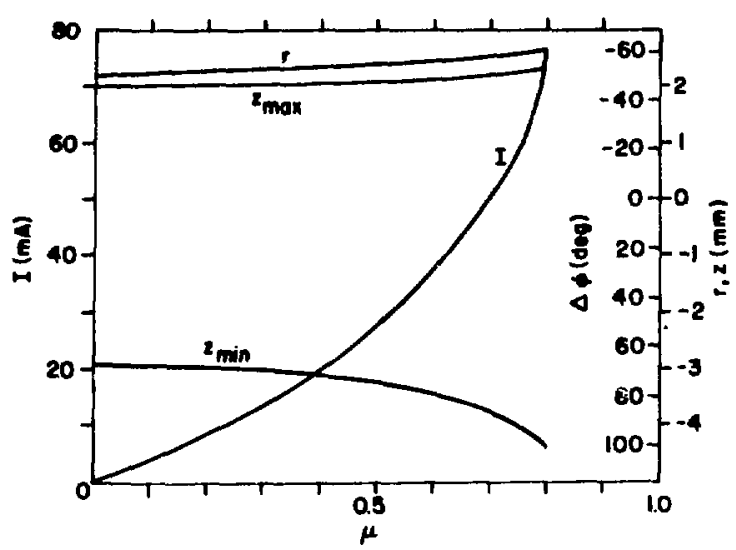

Fig. 82 .

(2) The current, radius, and minimum and maximum axial dimensions for a beam bunch matched to a smooth focusing linear accelerator containing the $z^{3}$ axial nonlinearity are shown as functions of the space charge paramenter $\mu$.

results in Fig. 82, in which we plot the radial and axial beam sizes and the current as a function of $\mu$. The parameter $\mu$ is the ratio of the space charge to external forces evaluated at the center and averaged over the three directions. This parameter is proportional to the central charge density. For low $\mu$, we can increase the current simply by increasing the phase-space density; therefore, the current is proportional to $\mu$. For larger $\mu$ values, the matched beam size grows so that the current increases faster than $\mu$. For the larger beams, the z-asymmetry is evident.

4. ATS Distributions

a. Current Limit. For the test-stand parameters, as in Table XVII, the highest current $\mu=0.8$ we found as above, except that we used both the rf coupling and the axial nonlinearity terms in the Hamiltonian. Table XVIII shows the results. There is little difference in the numbers from the case with no if coupling.

TABLE XVIII

ATS MAXIMUM CURRENT BEAM

RZED79 parameters

Current

Normalized emittance $(100 \%)$

Radius (100\%)

$z_{\min }\left(\Delta \phi_{\max }\right)(100 \%)$

$z_{\max }\left(\Delta \phi_{\min }\right)(100 \%)$ $\mu=0.8 \alpha=1 n=2$

$\delta=0.05 z^{3}, r^{2} z$ terms

$76.5 \mathrm{~mA}$

$0.31 \pi \mathrm{cm}$ mrad

$2.8 \mathrm{~mm}=8 \lambda / 5.7$

$-4.5 \mathrm{~mm}(1040)$

$2.3 \mathrm{~mm}\left(-53^{\circ}\right)$ 
b. Small Emittance Beam. Under the same conditions, but for $\delta=0.02$, we obtain the distribution shown in Table XIX. The emittance for $100 \%$ of the beam is $0.047 \pi \mathrm{cm} \cdot \mathrm{mrad}$. For $85 \%$ of the beam in one transverse direction, the emittance is $0.02 \pi \mathrm{cm} \cdot \mathrm{mrad}$.

E. Dynamics Results

We now present HOT particle-tracing results that involve nonlinearities and couplings in the external focusing forces.

1. Matched Beams. We start with the distribution of Table VIII; that is, a beam near the space-charge limit and large enough so that external nonlinearities are important. This beam is matched to take into account both the axial nonlinearity and the rf coupling forces. We traced this beam through $48 \mathrm{rf}$ cells of the accelerator whose initial parameters are as given by Table XVII. The transverse phase advance per cell was constant throughout. Figure 83a shows the three phase-space projections for the initial distribution. Figure $83 \mathrm{~b}$ shows the final distribution for an accelerator with acceleration turned off (the electric field provides axial focusing only). Figure $83 \mathrm{c}$ shows the final distribution for an accelerator with an electric field proportional to $\beta$ and a fixed synchronous phase angle. The final energy is $17.6 \mathrm{MeV}$. For this case, the linear part of the axial focusing force is constant but the nonlinear part decreases (phase damping is present, which means the axial potential well is growing in width). This change occurred adiabatically so we matched the final distribution to a structure with less nonlinearity. The bunch length is little changed but the axial phase-space distribution has lost its asymmetry about $z=0$ and is nearly elliptical. Figure 83d shows the final distribution for an accelerator with a fixed

Tabie XIX

ATS $\varepsilon=0.02 \pi \mathrm{cm} \cdot \mathrm{mrad}$ DISTRIBUTION

RZED79 parameters

Current

Normalized emittance (100\%)

Radius (100\%)

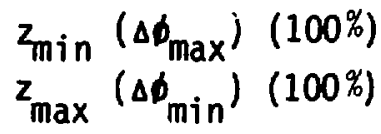

$\mu=0.8 \alpha=1 n=2$

$\delta=0.02 z^{3}, r^{2} z$ terms

$4.6 \mathrm{~mA}$

$0.047 \pi \mathrm{cm} \cdot \mathrm{mrad}$

$1.0 \mathrm{~mm}=\beta \lambda / 15$

$-1.2 \mathrm{~mm}\left(27^{\circ}\right)$

$0.99 \mathrm{~mm}(-23)$ 

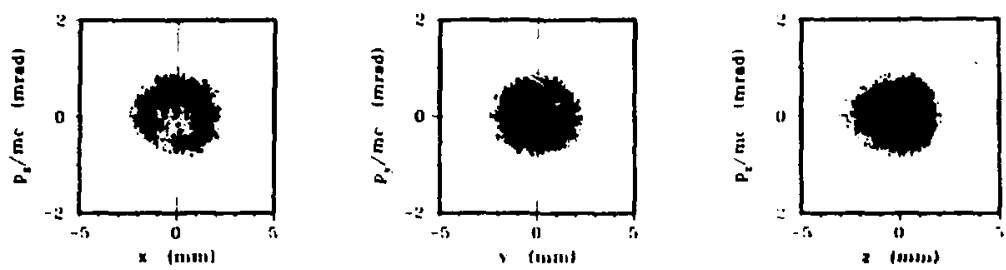

Fig. 83a.

Initial distribution for a beam matched to a smooth focusing linac containing the axial nonlinearity $\left(z^{3}\right)$ and $r f$ coupling $\left(r^{2} z\right)$.
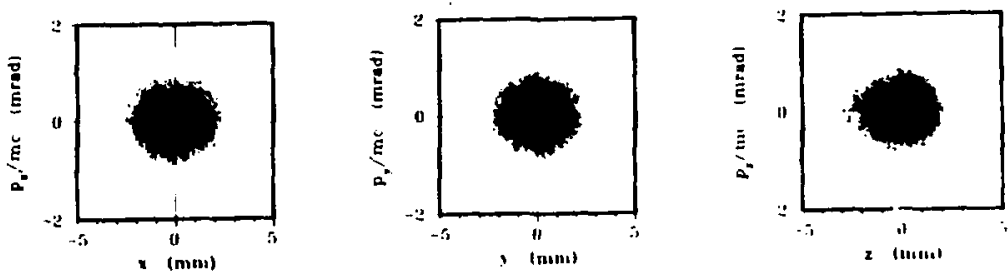

Fig. 83b.

Distribution after 48 cells with acceleration turned off. This distribution is similar to the initial one above.

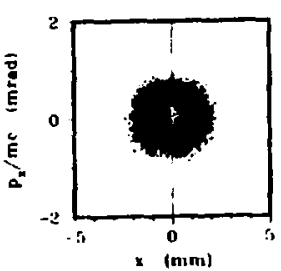

Distribution after 48 cells in an accelerator with accelerating field

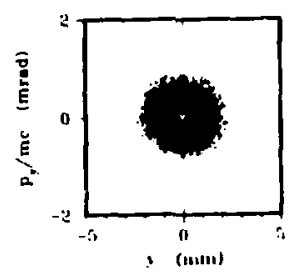

Fig. $83 c$.

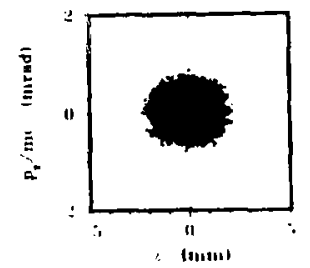
proportional to $B$. The linear part of the axial focusing force is fixed so the bunch length remains the same. Phase damping, which occurs even in the presence space charge, reduces the nonlinear part of the focusing forces so the axial phase space shape becames elliptical.
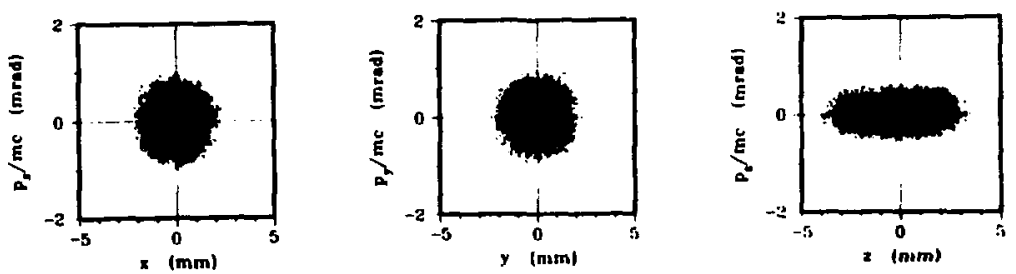

Fig. 83d.

Distribution after 48 cells in an accelerator with fixed accelerating field. Both the linear and nonlinear parts of the axial focusing forces have decreased. Consequently, the final bunch length is larger and the axial phase space shape is more elliptical than in the initial distribution. 
electric field and synchronous phase angle. The final energy is $2.5 \mathrm{MeV}$. The axial focusing force decreases so the bunch length increases. Even in this case, phase damping is present; that is, the bunch length increases slower than $B$ so that the nonlinear part of the force decreases faster than the linear part. Consequently, the axial phase-space distribution becomes elliptical. The emittances ( $90 \%$ values from ellipse-fitting) for the initial and the various final distributions are shown in Table $X X$.

We expected the lack of emittance growth for the no-acceleration case because of the initial equilibrium distribution. The lack of emittance growth for the other cases confirms that the focusing variation along the accelerator is sufficiently adiabatic.

2. $r f$ Coupling and Emittance Growth. The $r f$-coupling term $r^{2} z$ in the Hamiltonian can lead to emittance growth unless we prepare the initial distribution in a special way, because the transverse focusing forces depend on the 2-coordinate; that is, the front and rear parts of the bunch see different focusing forces in the transverse directions. To investigate this effect, we matched the $\mu=0.8$ distribution to the axial nonlinearity, but not the $r f$ coupling described in Figs. 81 and 82 . We accelerated this distribution for 48 rf cells in an accelerator with fixed transverse phase advances and a fixed electric field with the parameters shown in Table XXI. The initial phase-space projections are shown in Fig. 84a. Figure 84b shows the final distribution for the case in which rf coupling forces in the accelerator are turned off. This case was run to verify that the distribution is properly matched to the accelerator except for the $r f$ coupling effect. Figure $84 \mathrm{c}$ shows the final distribution for the case of a realistic accelerator with the rf coupling

TABLE XX

NORMALIZED 90\% EMITTANCE FOR MATCHED BEAM AFTER 48 CELLS

(in $\mathrm{cm} \cdot \mathrm{mrad}$ )
$1 / 2\left(\varepsilon_{x}+\varepsilon_{y}\right) / \pi$
$\varepsilon_{2} / \pi$
$\varepsilon_{x}+\varepsilon_{y}+\varepsilon_{z} / \pi$

Initial

0.151

0.158

0.460

No Acceleration

0.151

0.155

0.457

$E_{0} \propto$

0.151

0.149

0.450

$E_{0}=$ Const.

0.148

0.163

0.459 
TABLE XXI

NORMALIZED 90\% EMITTANCE FOR BEAMS NOT MATCHED TO

rf-COUPLING FORCES AFTER $48 \mathrm{CELLS}(\mathrm{cm} \cdot \mathrm{mrad})$.

$$
1 / 2\left(\varepsilon_{x}+\varepsilon_{y}\right) / \pi \quad \varepsilon_{z} / \pi \quad\left(\varepsilon_{x}+\varepsilon_{y}+\varepsilon_{z}\right) \pi
$$

$\begin{array}{llll}\text { Initial } & 0.148 & 0.159 & 0.454 \\ \begin{array}{l}\text { Accelerate without } \\ \quad \text { rf coupling }\end{array} & 0.146 & 0.167 & 0.458 \\ \text { Accelerate with rf } & 0.172 & 0.227 & 0.570\end{array}$

Coupling

included. The $90 \%$ (from ellipse fitting) normalized emittances for both cases are shown in Table XXI. The emittance growth caused by the rf coupling is substantial in both the transverse and axial directions. Compare this result to the almost negligible growth seen in the previous section (Fig. 83d and last line in Table $X X$ ) when we accelerated (in the same accelerator) a distribution with the proper correlations between the various directions in the 60 phase space.

3. Resonances and Three Dimensioris. Particle-tracing simulations have shown previously, before external nonlinearities were studied, that whenever the three phase-space projections did not have the same area, and in addition, when there was a mismatch or if variable parameters were involved, then the three areas approached each other with the sum remaining constant to very good accuracy. (Matched beams with unequal areas maintain the initial inequality if the focusing strengths do not change, as in an accelerator with $\left.E_{0} \propto \beta\right)$. When external nonlinearities were added in such situations, we found that this numerical experimental conservation law did not hold. This made analyzing rf-coupling effects or other sources of emittance growth difficult. It was not possible to distinguish "real growth" from a transfer of energy, or other quantity between different directions. At first, we suspected that the difficulty was merely in measuring the areas, because an ellipse-fitting procedure will not be accurate for nonelliptical distributions; therefore, we added the true-area (bin-counting) procedure to the particle-tracing code. This did not solve the problem. The accelerator of Table XVII that we use as 

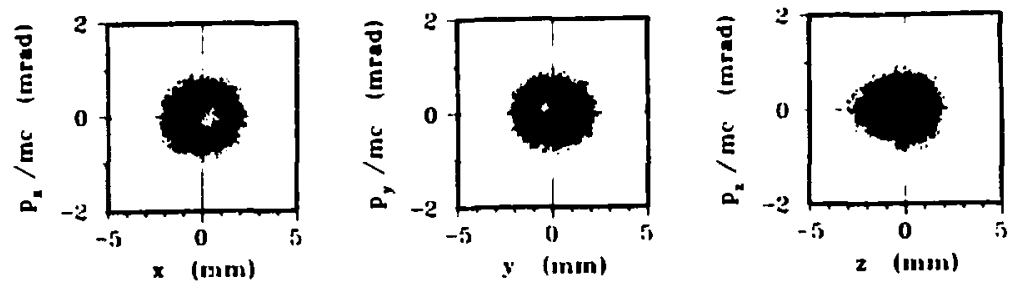

Fig. $84 a$.

Initial distribution for a beam matched to the axial nonlinearity but not the rf coupling of a smooth focused linac.
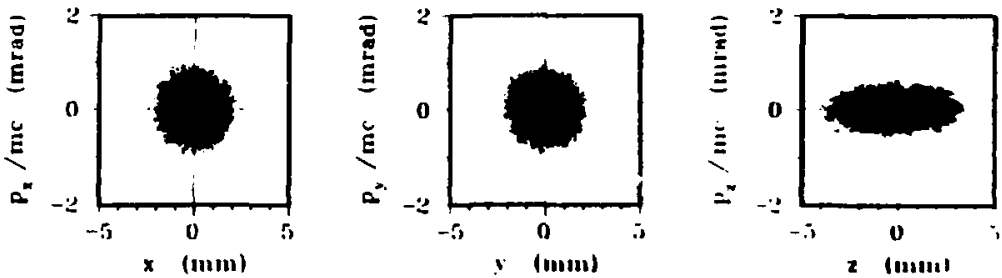

Fig. $84 b$.

Distribution after 48 cells in an accelerator with rf coupling turned off. The beam is matched to the accelerator.
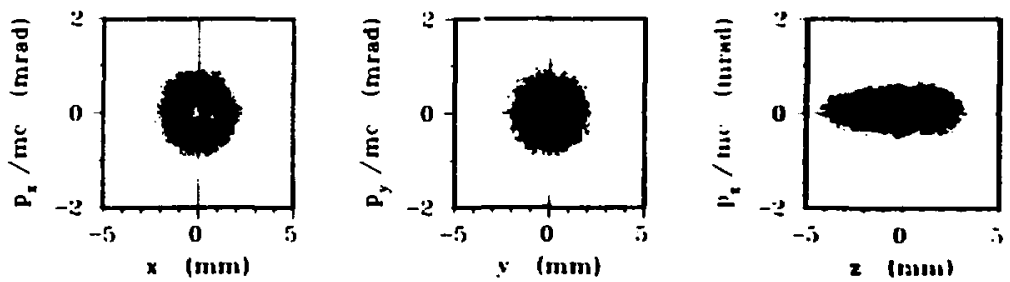

Fig. $84 c$.

Distribution after 48 cells in an accelerator that includes the $r f$ coupling forces. Because of the mismatch there is some emittance growth.

an example here has the same focusing strength in all directions, so that matched distributions have the same emittance in all three planes.

To investigate the existance of invariants in the presence of nonlinearities, we studied a problem that was nonstatic and could be solved analytically by perturbation theory. This was the weakly nonlinear motion near the $2 \mu_{x}-\mu_{z}=2 k$ resonance in the absence of space charge. This resonance is excited by the $x^{2} z$ part of the $r f$-coupling term $r^{2} z$. For the continuous focusing that we have in the HOT particle tracing code, only the $k=0$ harmonic is present so the resonance condition is $2 \mu_{x}-\mu_{z}=0$. With 
no space charge we can predict the motion if the perturbation is small enough (beam size is small enough). Also, without the need to calculate space charge, HOT can simulate this 30 problem. To prepare the distribution, we started with an RZED79 distribution having the following parameters: $\mu=0.05$ (RZED79 will not accept $\mu=0$ ), $a=1, n=2$, with no nonlinearities in the external focusing forces assumed. A current of $59 \mathrm{nA}$ in the accelerator of Table XVII corresponds to a matched beam radius of $0.05 \mathrm{~mm}$. With space charge, acceleration, and external nonlinearities turned of in HOT, we traced this distribution for 100 cells. During this time we reduced the $x$-focusing force so that the $x$-plane advance was reduced adiabatically from $90^{\circ}$ to $45.5^{\circ}$, then the resonant combination was $2 \mu_{x}-\mu_{z}=1^{0}$. This means that the perturbed amplitudes would oscillate in 360 focusing periods or 720 rf cells if there were a perturbation to excite this resonance. We traced the distribution for 100 more cells, keeping the phase advances fixed, to verify that the distribution matched these new parameters. Then we turned on the rf-coupling term and traced the distribution for 1440 more cells. In Fig. 85 we show the $90 \%$ emittances as a function of cell number. As we expected, the emittance in the y-direction was constant, to within 1\%. The oscillations have approximately the correct period of 720 cells. The sum of the three emittance values is more nearly constant compared to the $x$ and $z$ emittances.

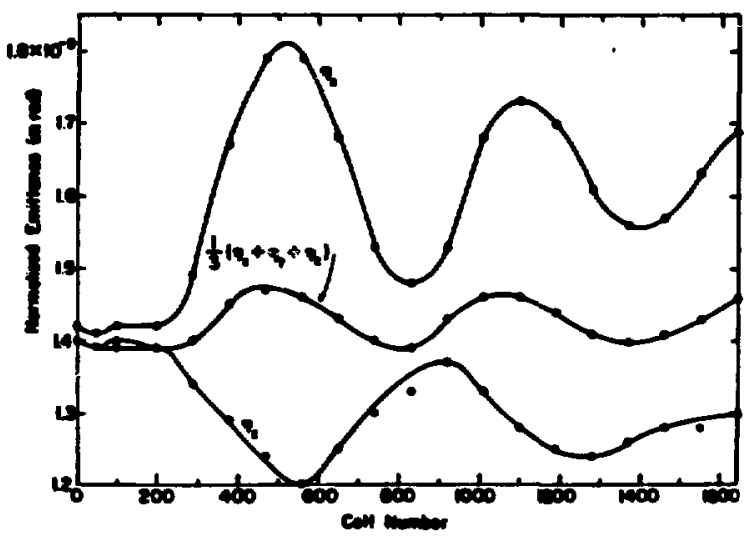

Fig. 85 .

The normalized emittances from a mismatched beam with no space charge in the vicinity of the $2 \mu_{X}-\mu_{Z}=0$ resonance are shown as functions of the cell number.
Assuming random phases in the initial distribution (that is, at cell 200) we predict that all emittances will oniy increase and return to their initial value after 720 cells but never decrease (as in the behavior of the emittance sum in Fig. 85). We feel that we can learn more from this experiment after further analysis. Some of this behavior may be caused by the particular way we have formed the distribution and may not be related to the existence of a useful invariant. Fortunately, the linear adiabatic 
nature of the single-particle motion will help us to understand the distribution that was formed.

F. Discussion

In the initial work on this subject we considered a smooth approximation with azimuthal symmetry. We found that high-brightness beans required very strong focusing systems, but we observed no fast-emittance growth in particle-tracing simulations. Phase damping was present even for beams near the space-charge limit. This year, we have found that discrete rf gaps do not modify these results. Because nonlinearities and couplings, arising from space charge alone, did not lead to emittance growth, we began the study of external nonlinearities in the smooth, azimuthally symetric model. We wrote the RZED79 code to generate the required phase-space distributions that we matched to an accelerator with axial nonlinearity and rf-coupling forces. In the particle-tracing simulations, we found emittance growth caused by $r f$ coupling but this emittance growth could be completely matched away with the right phase-space distribution. He still found phase danping and its presence caused the nonlinearities to decrease with acceleration.

Trying to understand why the sum of the three emittance values was no longer a good invariant under the condition that initial emittances were not equal, led us to study the $2 \mu_{x}-\mu_{z}=0$ resonance, which is excited by the rf-coupling forces. Although this is a 30 problem, we were able to study it with the existing numerical apparatus if we neglected space charge. Simulation of alternating gradient focusing is a 30 time-dependent problem and we are developing a 30, space-charge routine. At present, we can study some 30, time-dependent features of linac dynamics, wich do not require space charge. We will study periodic-equilibrium distributions by adiabatically deforming distributions generated by RZED79, for example, before 3D, space-charge calculations will be possible.

\section{THE STARK EFFECT AS A TOOL FOR MEASURING RF ELECTRIC FIELDS}

The accurate measurement of electric fields in $r f$ cavities is a difficult problen. Three different techniques have been used. The first technique calibrates electric fields by comparison with a computer code such as SUPERFISH. This approach is difficult because it is indirect and thus involves various assumptions, in particular about geometry, that may not be satisfied. The second technique calibrates by using diffusion-controlled 
breakdown of gases. There are two difficulties with this approach: (1) The geometry usually is not parallel plate, which is the only geometry for which we have standardized measurements. (2) Breakdown is often surface controlled, and not diffusion controlled, a more difficult problem. The diffusion-

controlled breakdown regime requires high frequency (multigigahertz) and large scales (several centimeters); rf cavities for proton linacs usually are not in this regime. The third technique measures electric fields by stimulating emission of electrons with a laser and observing the spectrum of emitted $x$ rays. This method is a direct, but unfortunately crude, measurement of the electric field.

There is a fourth technique for measuring rf electric fields that we wish to discuss. This technique's advantages are that it is direct and potentially is very accurate. Even with crude measurements, the technique can be used as an independent check of the stimulated electron-emission method. Our basic idea is to observe the Stark shift of spectral lines of a cavity-containing gas. There are two possibilities: the linear Stark effect and the quadratic Stark effect.

The advantage of using the linear Stark effect is that when it exists, as in atomic hydrogen, it is large. However, because we are concerned with $r f$ fields, a simple line shift is not observable. There are two possibilities: the period of the $r f$ may be longer or shorter than the lifetime (natural or collisional) of the observed quantum state. If the $r f$ period is shorter than the lifetime of the quantum state, the linear Stark effect vanishes because of time averaging. If the $r f$ period is longer than the lifetime of the state, a broadening of the line will be observed if the observation interval is longer than the rf period (the only practical case). Because the natural lifetimes of most states exceed $10 \mathrm{~ns}$, we cannot observe the line broadening in a low-pressure gas at high frequency ( $>100 \mathrm{MHz}$ ). In a high-pressure gas, in which the collision lifetime is less than the rf period, we may be able to accurately measure the line broadening. The collisional lifetime varies from state to state and may be very different from the collision time based on the total cross section. Note also that if we initiate a discharge, the stark broadening that is due to electrons and ions may doninate the Stark broadening that is due to if fields. 
The quadratic Stark effect is smaller than the linear Stark effect, but has the advantage that it does not average to zero. The quadratic Stark effect also has two regimes; that is, the rf period can be either shorter or longer than the lifetime of the observed state. ${ }^{101}$ If the $\mathrm{rf}$ period is longer than the lifetime, again we will observe line broadening. This case is like that for the linear Stark effect and similar comments apply. If the rf period is shorter than the lifetime, then a simple line shift will be observed as in the static Stark effect. However, because of time averaging, the magnitude of the shift will be half that of the static shift for the same E-field; that is, $E$ acts like $E / \sqrt{2}$. The intermediate regime, in which the lifetime of the quantum state is comparable to the rf period, is very complicated and probably is of no use in measuring E-fields.

Two experimental techniques are used for measuring the Stark effect. The first technique looks at absorption spectra, using a tunable laser. This technique has many advantages. (1) Spatial localization is comparatively easy. (2) The measurement requires no gas breakdown, with attendant ionization Stark broadening at high pressure. Also, because breakdown is not required, the measured rf electric field can be varied easily. Finally, the polarization of the incident light can be varied accurately, thus allowing excitation of only certain locales in the gas, because the Stark transitions are sensitive to polarization. The principal disadvantage of this technique is its complexity.

The second technique for measuring the Stark effect is to initiate a glow discharge and to look at the emission spectra. This technique has been used for static fields ry Dalby ${ }^{102}$ and coworkers to measure dipole moments of various molecules. The principal advantage of this technique is its simplicity: one simply passes the spontaneously emitted light through a spectrograph for analysis. This technique should be quite satisfactory for measuring the maximum electric field, but has disadvantages related to its lack of detail. Only the electric field in the region of the glow can be measured and this field can be changed only by shifting the breakdown point by a pressure change. It is probably hard to observe the line broadening, either linear or quadratic. Besides the inherent difficulty of defining the line width, the required high pressure probably precludes breakdown; even if breakdown occurs, the ionization Stark broadening probably will dominate the 
effect. Probably line widths can only be measured by difficult absorption experiments. A final difficuity with the interpretation of the glow discharge experiment is electron loading. Although the electric field still will be determined absolutely, its primary purpose will be calibration against rf power and this calibration will be affected by electron loading.

Thus, the most likely candidate is the line shift that is due to the quadratic Stark effect. Low pressures are required to observe the effect. Molecular hydrogen probably will suffice as the gas. The quadratic Stark effect in hydrogen has been observed by Sne $17^{103}$ for many lines in the visible. At $400 \mathrm{MHz}$, we require a lifetime in excess of $10 \mathrm{~ns}$ to be in the line-shift regime, which should be satisfied.

The magnitudes of the expected shifts can be derived from Snell. 103 In a field of $9 \mathrm{MV} / \mathrm{m}$, the lin: at $6019.95 \AA$ was shifted by $8.1 \mathrm{~cm}^{-1}$ (2.93A). Many other lines in the same range were silifted by anounts froin $i \mathrm{~cm}^{-1}$ to $10 \mathrm{~cm}^{-1}$. These shifts are easily detected by the larger spectrographs, but may be difficult for smaller, portable spectrographs.

The data interpretation is complicated by several facts. Because the E-field is usually spatialiy inhomogeneous, and light from regions of different E-field will reach the detector, sharp lines will not be observed. However, if the E-field magnitude inhomogeneity is correlated with direction inhomogeneity, polarization will sharpen the lines and we should be able to compute the maximum magnitude of the E-field from the maximum extent of the line. In the more elaborate absorption experiment, the polarization effect probably can be used to obtain detailed field maps. 
1. J. D. Schneider, H. L. Rutkowski, E. A. Meyer, D. D. Armstrong, B. A. Sherwood, and L. L. Catlin, "Development of a High-Current Deuteron Injection for the FMIT facility," Proc. 10th Linear Accelerator Conf., Montauk, NY, September 10-14, 1979 (Brookhaven Nat. Laboratory, Upton, NY, 1980) BNL-51134.

2. I. M. Kapchinskii and V. A. Teplyakov, "Linear Ion Accelerator with Spatially Homogeneous Strong Focusing," Nuclear Experimental Techniques, 1970 Consultants Bureau, Plenum Publi shing Corp., report UDC 621.384.64 (1970).

3. D. A. Swenson, "Low-Beta Linac Structures," Proc. 10th Linear Accelerator Conf., Montauk, NY, September 10-14, 1979 (Brookhaven Nat. Laboratory, Upton, NY, 1980) BNL-51134.

4. R. H. Stokes, K. R. Crandall, J. E. Stovall, and D. A. Swenson, "RF Quadrupole Beam Dynamics," IEEE Trans. Nucl. Sci. NS-26, 3469 (June 1979).

5. K. R. Crandall, R. H. Stokes, and T. F. Wangler, "RF Quadrupole Beam Dynamics Design Studies," Proc. 10th Linear Accelerator Conf., Montauk, NY, September 10-14, 1979 (Brookhaven Nat. Laboratory, Upton, NY, 1980) BNL-51134.

6. K. R. Crandal1, R. H. Stokes, and T. P. Wangler, "RFQ Beam Dynamics and Pole Tip Geometry for the 425-MHz Proof of Principle Test," AT Division memo, 8/9/79, LASL internal document.

7. M. V. Fazio, H. P. Johnson, D. M. Riggin, "Developments on the rf System for the Fusion Materials Irradiations Test Accelerator," Proc. 10th Linear Accelerator Conf., Montauk, NY, September 10-14, 1979 (Brookhaven Nat. Laboratory, Upton, NY, 1980) BNL-51134.

8. J. A. Johnson, D. R. Machen, R. M. Suyama, "Distributed Control System for the FMIT," Proc. 10th Li near Accelerator Conf., Montauk, NY, September 10-14, 1979 (Brookhaven Nat. Laboratory, Upton, NY, 1980) BNL-51134.

9. R. A. Jameson, "High-Intensity Deuteron Linear Accelerator (FMIT), Proc. 1979 Particle Accelerator Conf., San Francisco, CA, March 12-14, 1979 IEEE Trans. Nucl Sci. NS-26, No. 3, June 1979.

10. E. L. Kemp, D. J. Liska, M. D. Machalek, "The Fusion Materials Irradiation Test (FMIT) Accelerator," Proc. 10th Linear Accelerator Conf., Montauk, NY, September 10-14, 1979 (Brookhaven Nat. Laboratory, Upton, NY, 1980) BNL-51134.

11. D. J. Liska, R. G. Schamaum, W. Fox, J. A. Frank, W. Coops, C. Potter, T. Cole, D. Greenwood, H. Norman, P. Giles, K. Creek, "Modular Design Aspects of the FMIT Drift Tube Linac," Proc. 10th Linear Accelerator Conf., Montauk, NY, September 10-14, 1979 (Brookhaven Nat. Laboratory, Upton, NY, 1980) BNL-51134. 
12. G. P. Boicourt and R. A. Jameson, "A Statistical Approach to the Est imation of Beam Spill," Proc. 10th Linear Accelerator Conf., Montauk, NY, September 10-14, 1979 (Brookhaven Nat. Laboratory, Upton, NY, 1980) BNL-51134.

13. G. P. Boicourt and D. A. Swenson, "Choice of Geometry for the Alvarez Section of the FMIT Deuteron Linac," IEEE Trans. on Nucl. Sci., Vol. NS-26 No. 3, June, 1979.

14. D. J. Liska, R. G. Schamaun, C. Potter, C. Fuller, D. Clark, D. Greenwood, and J. A. Frank, "Design of the Accelerating Structures for FMIT," IEEE Trans. Nucl. Sci. NS-26, No. 3, pp. 3052-3054 (June 1979).

15. K. E. Melson, J. D. Farre11, D. J. Liska, "A High-Energy Beam Transport System," 1979 Linac Conf., Proc. 10th Linear Accelerator Conf., Montauk, NY, September 10-14, 1979 (Brookhaven Nat. Laboratory, Upton, NY, 1980) BNL-51134.

16. Daniel D. Hudgings and W. Folkner, "Response of an Inductive Detector to a Pulse Train," PSR Tech. Note 27, 7-16-79, LASL internal document.

17. W. Folkner, "Beam Simulation and Position Monitor Reponse," PSR Tech. No. 39, 12-4-79, LASL internal document.

18. D. W. Hudgings, "Beam Profile Measurements by Background Gas Ionization," PSR Tech. No. 26, 7-10-79, LASL interilal document.

19. E. Higgins, "PSR Beam Monitoring System and Initial Tuneup Scheme," PSR Tech. Note $36,11-5-79$, LASL internal document.

20. T. W. Hardek and W. E. Chyna, "Common Anode Amplifier Development," IEEE Trans on Nucl Sci, Vol NS-26, No. 3, June 1979, p 3959.

21. M. Donald, "Fundamental Mode Beam Loading in the 603.75 MHz rf System," PSR Tech. Note 37, 5-79, LASL internal document.

22. P. Sikora, "Formal Calculation of Cavity Responses to On-Axis Beam Evaluation for Lowest Modes Only," PSR Tech. Note. 41, 8-79, LASL internal document.

23. K. W. Robinson, "Stability of Beam in Radio-Frequency System, "Cambridge Electron Accelerator Laboratory report CEAL-101C.

24. S. 0. Schriber, "Disk-and-Washer Structure for PSR High-Frequency Buncher, "PSR Tech. Note 31, 8-6-79, LASL internal document.

25. M. C. Thompson, "Experience with the LAMPF Line D Fast-Kicker System," 1979 Particle Accelerator Conf., March 12-14, 1979, San Francisco.

26. D. W. Hudgings, "Bump-Magnet Power Supply," PSR Tech. Note 34, 10-15-79, LASL internal document. 
27. W. Folkner, "Time-of-Flight Energy Measurement," PSR Tech. Note 33, 9-28-79, LASL internal document.

28. D. A. Clark, et. al., "Time-Resolved Beam Energy Measurements at LAMPF," 1979 Particle Accel. Conf., March 12-14, 1979, San Francisco.

29. "Alexander J. Dragt, "A Method of Transfer Maps for Linear and Nonlinear Beam Elements," to be published in the Proc. of the 1979 Particle Accelerator Conf., San Francisco, CA, March 12-14, 1978.

30. Alexander J. Dragt, "Transfer Map Approach to Beam-Beam Interactions," Proc. of Symp. on Nonl inear Dynamics and The Beam-Beam Interaction held at Brookhaven National Laboratory, March 19-21, 1979.

31. G. Spalek, "PSR Beam-I nduced Desorption," PSR Tech. Note No. 19, 2-26-79, LASL internal document.

32. A. Jason, "Foil Stripper Design Considerations," PSR Tech. Note. 29, 8-2-79, LASL internal document.

33. R. C. Webber and C. Hojvat, "Measurements of the Electron Cross Sections for Negative Hydrogen Ions on Carbon at $200 \mathrm{MeV}, "$ IEEE Trans. on Nucl. Sci. NS-26, 4012 (1979).

34. J. H. W. Simmons, Radiation Damage in Graphite, (Pergammon, Oxfor $u$, 1965).

35. J. L. Yntema, "Lifetimes of Carbon Stripping Foils," IEEE Trans. on Nucl. Sci. NS-23, 133 (1976).

36. N. R. S. Tait, D. W. L. Toltree, D. S. Whitewell and B. H. Armitage, Daresbury and Harwell, "The Behavior and Physical Characteristics of Carbon Stripper Foils Prepired by Different Methods," prepublication results.

37. H. A. Bethe, "Molieres Theory of Multiple Scatering," Phys. Rev. 891256 (1953).

38. R. K. Cooper and G. P. Lawrence, Beam Emittance Growth in a Proton Stroage Ring Employing Charge Exchange Injection," IEEE Trans. on Nucl. Sci. NS-22, 1996 (1975).

39. A. E. Livingston, H. G. Berry, and G. E. Thomas, "Thin Carbon Foil Breakage Times Under Ion Beam Bombardment," Nucl. Instr. and Methods 148,125 (1978).

40. A. C. Paul, "Proton Storage Ring Injection Line Calculations," PSR Tech. Note 25, 7-79, LASL internal document.

41. A. C. Paul, "Proton Storage Ring Extraction Line Calculations," PSR Tech. Note. 16, 7-31-79, LAS internal document. 
42. D. W. Hudgings, "Neutral Beam Injection for a Proton Storage Ring," 1979 Particle Accelerator Conf., March 1.2-14, 1979, San Francisco.

43. T. Kwan, J. M. Dawson and A. T. Lin, Phys. Fluids 20, 581 (1977).

44. N. M. Kroll and W. A. McMullin, Phys. Rev. A 17, 300 (1978).

45. P. Sprangle, C. M. Tang, and W. M. Manheimer, "The Non-Linear Theory of Free Electron Lasers and Efficiency Enhancement," Naval Research Laboratory memorandum, report 4034 (1979).

46. M. Zales Caponi, J. Munch, and H. Boehmer, "Optimized Operation of a Free Electron Laser, Spanning the Single Particle and Collective Regimes," Workshop on Free Electron Generators of Coherent Radiation, Telluride, Colorado, August 13-17, 1979.

47. C. Pellegrini, "Synchrotron Radiation Problems in Storage Ring Version of FEL," Workshop on Free Electron Generators of Coherent Radiation, Telluride, Colorado, August 13-17, 1979.

48. T. I. Smith, J. M. J. Madey, L. R. Elias, and D. A. G. Deacon, J. Appl. Phys. 50, 4580 (1979).

49. N. M. Kroll, P. L. Morton, and M. N. Rosenbluth, "Variable Parameter Free Electron Laser," Workshop on Free Electron Generators of Coherent Radiation, Telluride, Colorado, August 13-17, 1979.

50. L. R. Elias, Phys. Rev. Lett. 42 , 977 (1979).

51. F. A. Hopf, P. Meystre, M. O. Scully, and W. H. Loui sell, Opt. Commun. $\underline{18}, 413$ (1976).

52. F. A. Hopf, P. Meystre, M. 0. Scully, and W. H. Loui se11, Phys. Rev. Lett. 37, 1342 (1976).

53. P. Meystre, G. T. Moore, M. 0. Scully, and F. A. Hopf (in press).

54. C. A. Brau, IEEE J. Quantum Electron (in press).

55. K. Halbach, Lawrence Berkeley Laboratory, private communication.

56. J. Elliot, Los Alamos Scientific Laboratory, private comunication.

57. S. J. Czuchlewski, E. J. Mclellan, J. F. Figueira, E. Foley, C. E. Knapp, and J. A. Webb, Proc. of the Int. Corf. on Lasers, 1978, Orlando, Florida, Dec., 1978.

58. E. J. McLellan and J. F. Figueira, Rev. Sci. Instrum. 50, 1213 (1979). 
59. D. H. Gill and B. E. Newnam, Digest of Post-Deadline Papers, Inertial Conf inement Fusion Conf., San Diego, Feb. 7-9, 1978.

60. G. Friedlander, Chairman, "Future of Nuclear Science," report of the Ad Hoc Panel on the Future of Nucl. Sci., Nat. Acad. of Sci., Washington, DC, 1977.

61. "The Role of Electron Accelerators in US Medium Energy Nuclear Science," report of DOE/Nat. Sci. Foundation Study Group, R. S. Livingston, Chairman, ORNL/PPA-77/4, December 1977.

62. V. A. Volodin and A. 0. Hanson, IEEE Trans. on Nucl. Sci. NS 22, No. 3, 1194 (1975).

53. "Beam Blowup in Race Track Microtrons," H. Herminghaus, Institute fur Kernphysik, Mainz University internal report KPH15/78 (May 1978).

64. B. H. Wick and P. B. Wilson, Nucl. Instr. and Methods 56, 197 (1967).

65. H. Herminghaus, A. Feder, K. H. Kaiser, W. Manz, and H. v.d. Schmitt, Nucl. Instr. and Methods 138, 1 (1976).

66. J. M. Potter, S. 0. Schriber, and F. J. Humphry, IEEE Trans. on Nucl. Sci. NS-26, 3, 3763 (1979).

67. T. P. Wangler, "Space Charge Limits in Linear Accelerators," to be published.

68. T. P. Wangler and R. H. Stokes, "Application of the RF Quadrupole in Linear Accelerators for Heavy Ion Fusion," Proc. of the 1979 Heavy Ion Fusion Workshop, Berkeley CA (Oct. - Nov., 1979).

69. D. A. Swenson, "RF Linac Approach to Heavy Ion Fusion," Proc. of the 1979 Heavy Ion Fusion Workshop, Berkeley, CA (Oct.-Nov. 1979).

70. P. J. Tallerico, "The Gyrocon, A Deflection-Modulated, High-Power Microwave Amplifier," Los Alamos Scientific Laboratory report LA-6907 (October 1977).

71. P. J. Tallerico, "A Class of Deflection Modulated, High-Power Microwave Amplifiers," 1977 Int. Electr Device Meeting Technical Digest, pp. 242-245, Washington, DC, December 1977.

72. G. I. Budker, M. M. Karliner, I. G. Makarov, S. N. Morozov, 0. A. Nezhevenko, G. N. Ostrieko and I. A. Shekhtman, "The Gyrocon--A Highly Efficient Converter of Energy from Powerful Relativistic Beams for Microwave Supplies in Charged-Particle Accelerators, "Atomic Energy, Vol. 44, No. 5, pp. 459 466, May 1978. 
73. G. I. Budker, M. M. Karliner, I. G. Makarov, S. N. Morosov, 0. A. Nezhevenko, G. N. Ostreiko and I. A. Shekhtman, "The Gyrocon-An Efficient Relativistic High Power VHF Generator," Particle Accelerators, Vol. 10, pp. 41-59, 1979.

74. G. I. Budker et al., "The $r f$ System of the VEPP-4 Electror-Position Storage Ring Based on the Gyrocon-A High-Power UHF Generator with an Unbunched Relativistic Beam," Dubna Accelerator Conf. Proc., Dubna USSR, October 1976. (Available in English as SLAC Trans-177, SLAC, August 1977.)

75. P. J. Tallerico and J. E. Rankin, "Computer Modeling of the Gyrocon," IEEE Trans. on Nucl. Sci., Vol. NS-26, No. 3, pp. 4015-4017, June 1979.

76. P. J. Tallerico and J. E. Rankin, "The Gyrocon; A High Efficiency, High-Power Microwave Amplifier," IEEE Trans. on Electron Devices, Vol. Ed-26, No. 10, pp. 1559-1566 (October 1979).

77. J. E. Rankin and P. J. Tallerico, "S-Band Microwave Power Generation with Spherical Gyrocons," submitted to the Space Solar Power Review, February 1980.

78. P. J. Tallerico and J. E. Rankin, "The Gyrocon RF-Generator Project for FY78 and - 79," LoS Alamos Scientific Laboratory report LA-8537-SR (September 1980).

79. W. B. Herrmannsfeldt, "Electron Trajectory Program," Stanford Linear Accelerator Center publication SLAC-166 (1973)

80. G. MacMaster and L. Nichols, "High Gain Crossed Field Amplifier Tube," Paper 12.4, Technical Digest, 1977 Int. Electron Devices Meeting, pp. 245B, December 1977, Washington, D C

81. D. J. Liska, "Multipactoring Electron Gun for High Duty Linacs," Proc. IEEE, Yol. 59, pp. 1253-1254, August 1971.

82. J. V. Lebacqz, A. J. Dudas, and W. R. Fowkes, "The Trirotron," IEEE Trans. Nucl. Sci., Vol. NS-26, pp. 3891-3893, June 1979.

83. P. W. Allison, "Experiments with a Dudnikov-Type $H^{-}$Ion Source," Proc. of the Symp. on the Production and Neutralization of Negative Hydrogen Ions and Beams, Brookhaven National Laboratory, New York, Sept. 26-30, 1.977, BNL-50727 (January 1978) p. 119-122.

84. AT-2 progress report, LASL internal document.

85. H. V. Smith, Jr. and P. W. Allison, IEEE Trans. on Nucl. Sci. NS-26 (1979) 4006 . 
86. J. B. Taylor and I. Langmuir, Phys. Rev. 44 (1933) 423.

87. Yu I. Bel'chenko, V. I. Davydenko, G. E. Derevyankin, A. F. Dorogov, and V. G. Dudnikov, Pis'ma Zh. Tekh. Fiz. 3 (1977) 693.

88. F. W. Sears, Thermodynamics, Add i son-Wesley, 1953, p. 241.

89. Karl L. Brown, "A First and Second Order Matrix Theory for the Design of Beam Transport Systems and Charged Particle Spectrometers," Adv. in Particle Physics 1 (1967) p. 71-134.

90. J. D. Sherman and P. W. Allison, "A Study of a $90^{\circ}$ Bending Magnet for $\mathrm{H}^{-}$Beams," IEEE Trans. on Nucl. Sci. NS-26 (1979) p. 3916-3918.

91. K. L. Brown, F. Rothacker, D. C. Carey, and Ch. Iselin, "A Computer Program for Designing Charged Particle Beam Transport Systems," SLAC-91, Rev. 2 (May 1977)

92. W. Joho and C. Kost, "Speam, A Computer Program for Space Charge Beam Envelopes," Triumf Design Note No. TRI-DN-73-11, University of British Columbia, Vancouver 8, BC, Canada, p. 1-19.

93. Jinchoon Kim, J. H. Whealton, and Gerd Schilling, "A Study of Two-Stage Ion-Beam Optics," J. App 1. Phys. 49 (2) (1978) p. 517-524.

94. Jack E. Boers, "Computer Simulation and Experimental Results for a $130 \mathrm{kV}, 80 \mathrm{~mA}$ Deuterium 9 Ion 9 Beam," R. A. Jame son, Ed., Space Charge in Linear Accelerators Workshop, Los Alamos Scientific Laboratory report [A-T265-C, (1978) p. 51-58.

95. Paul W. Allison, "Third Order Calculations of 200 kV Column Optics," LASL internal document.

96. R. Booth, J. C. Davis, C. L. Hanson, J. L. Held, C. M. Logan, J. E. Osher, R. A. Nickerson, B. A. Pohl and B. J. Schumacher, "Rotating Target Neutron Generators," Nucl. Instrum. and Methods $\underline{145}$ (1977) p. 25-39.

97. L. L. Alston, High Voltage Technology, L. L. Alston, Ed.(Oxford University Press 1968) p. 5-6.

98. J. L. Rand, R. W. Leep, and W. A. Bradley, "Design of Safe Electrical Equipment for Research," Los Alamos Scientific Laboratory report 7597, (1979) p. 12.

99. W. P. Lysenko, "Linac Particle Tracing Simulations," IEEE Trans. Nuc. Sci., NS-26, 3508 (1979).

100. W. P. Lysenko, "Equilibrium Phase-Space Di $t$ b ti ns nd Space Charge Limits in Linacs," Los Alamos Scientific Laboratory report LA-7010-MS (October 1977). 
101. C. H. Townes and A. L. Schawlow, Microwave Spectroscopy, Dover Publication, New York, NY (1975).

102. (a) D. H. Phelps F. W. Dalby, Can. J. of Phys., 43, 144, (1965)

(b) T. A. R. Irwin F. W. Dalby, Can. J. of Phys., 43, 1766, (1965)

(c) D. H. Phelps F. W. Dalby, Phys. Rev. Lett., 16, 13, (1966)

(d) R. Thomson F. W. Dalby, Can. J. of Phys., 46, 2815. (1968)

(e) C. Carlone F. W. Dalby, Can. J. of Phys., 47, 1945, (1969)

(f) R. Thomson F. W. Dalby, Can. J. of Phys., 47, 1155, (1969)

(g) E. A. Scarl F. W. Dalby, Can. J. of Phys., 49,2825, (1971)

(h) E. A. Scarl F. W. Dalby, Can. J. of Phys., 52, (1974)

103. H. Snell, Trans. Roy. Soc. 234, 115, (1935) 